\title{
Rate Acceleration in Olefin Metathesis through a \\ Fluorine-Ruthenium Interaction
}

Tobias Ritter, Michael W. Day, and Robert H. Grubbs*

Supporting Information 


\section{Materials and Methods.}

All reactions involving metal complexes were conducted in oven-dried glassware under a nitrogen atmosphere using standard glovebox techniques. Solvents were purified by passage through alumina. ${ }^{1}$ Resonances for NMR spectra are reported relative to $\mathrm{Me}_{4} \mathrm{Si}(\delta 0.0)$ for ${ }^{1} \mathrm{H}$ and ${ }^{13} \mathrm{C}$, and $\mathrm{H}_{3} \mathrm{PO}_{4}(\delta 0.0)$ for ${ }^{31} \mathrm{P}$. Spectra are reported as follows: chemical shift $(\delta \mathrm{ppm})$, multiplicity, coupling constant $(\mathrm{Hz})$ and integration. 2,6-Difluoroanniline, oxalyl chloride, $\mathrm{BH}_{3} \cdot \mathrm{THF}$ (1 M in THF), triethylorthoformate, and silver(I) oxide were purchased from Aldrich and used without prior purification. Catalyst $\mathbf{4}$ and $o$-isopropoxy- $\beta$-methylstyrene were obtained from Materia, Inc.

\section{Experimental Procedures}<smiles>O=C(Nc1c(F)cccc1F)C(=O)Nc1c(F)cccc1F</smiles>

S1

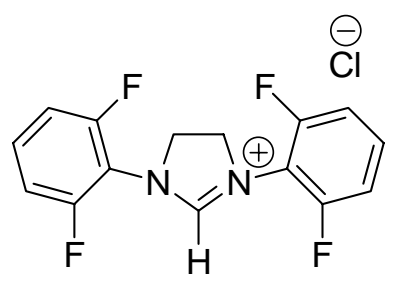

4

\section{Tetrafluoro diamide S1.}

To 2,6-difluoroanniline (20.7 g, $160 \mathrm{mmol}, 2.00$ equiv) in THF/water $(1: 1,160 \mathrm{ml})$ at $20{ }^{\circ} \mathrm{C}$ is added powdered $\mathrm{NaOH}(8.02 \mathrm{~g}, 200 \mathrm{mmol}, 2.50$ equiv) and triethylamine $(810 \mathrm{mg}, 8.00 \mathrm{mmol}$, 0.10 equiv). To this suspension is added oxalyl chloride (12.2 g, $96.2 \mathrm{mmol}, 1.20$ equiv) dropwise. The suspension is vigorously stirred for $30 \mathrm{~min}$, then poured onto water $(600 \mathrm{ml})$. The solids are filtered off, washed with $1 \mathrm{M} \mathrm{HCl}(100 \mathrm{ml})$, water $(3 \times 100 \mathrm{ml})$ and diethylether $(2 \times 100 \mathrm{ml})$ to afford a colorless solid $(18.7 \mathrm{~g}, 75 \%$ yield $)$.

Melting Point: mp $276{ }^{\circ} \mathrm{C}$ (toluene). NMR Spectroscopy: ${ }^{1} \mathrm{H}$ NMR (300 MHz, DMSO-d-6) $\delta$ : $10.79(\mathrm{~s}, 2 \mathrm{H}), 7.51-7.41(\mathrm{~m}, 2 \mathrm{H}), 7.28-7.21(\mathrm{~m}, 4 \mathrm{H}) .{ }^{13} \mathrm{C}$ NMR (75 MHz, DMSO-d-6) $\delta: 158.5$, 157.9 (dd, $J=249.9 \mathrm{~Hz}, 5.0 \mathrm{~Hz}), 129.2(\mathrm{t}, J=9.9 \mathrm{~Hz}), 113.3$ (t, $J=17.0 \mathrm{~Hz}), 112.2-111.9(\mathrm{~m})$, 66.4. ${ }^{19} \mathrm{~F}$ NMR (282 MHz, DMSO-d-6) $\delta:-117.7$. Mass Spectrometry HR-FAB NBA Calcd for $\mathrm{C}_{14} \mathrm{H}_{9} \mathrm{~N}_{2} \mathrm{O}_{2} \mathrm{~F}_{4}, 313.0600$. Found, 313.0601. Combustion Analysis: Anal. calcd for $\mathrm{C}_{14} \mathrm{H}_{9} \mathrm{~N}_{2} \mathrm{O}_{2} \mathrm{~F}_{4}$ : C, 53.86; H, 2.58; N, 8.97; F, 24.34. Found C, 53.55; H, 2.85; N, 8.77.

\section{Tetrafluorodihydroimidazolium chloride 4.}

${ }^{1}$ Pangborn, A. B.; Giardello, M. A.; Grubbs, R. H.; Rosen, R. K.; Timmers, F. J. Organometallics 1996, 15, 5181520 . 
To tetrafluoro diamide $\mathbf{S} 1$ ( $8.00 \mathrm{~g}, 25.6 \mathrm{mmol}, 1.00$ equiv) in a pressure tube is added $\mathrm{BH}_{3} \cdot \mathrm{THF}$ complex in THF $\left(1.0 \mathrm{M}, 152 \mathrm{ml}, 6.0\right.$ equiv). The solution is heated at $75{ }^{\circ} \mathrm{C}$ for $16 \mathrm{~h}$. After cooling, the clear, colorless solution is poured onto $200 \mathrm{ml}$ methanol containing $7 \mathrm{ml} \mathrm{HCl}$ (aq.) conc. The solution is concentrated in vacuo, subsequently, $200 \mathrm{ml}$ methanol is added. The solution is concentrated in vacuo to afford a colorless solid. A suspension of this solid in triethylorthoformate $\left(75 \mathrm{~g}, 85 \mathrm{ml}, 512 \mathrm{mmol}, 20\right.$ equiv) is heated at $135{ }^{\circ} \mathrm{C}$ for $30 \mathrm{~min}$. After cooling, the solids are filtered off and washed with diethylether $(3 \times 50 \mathrm{ml})$ to afford a colorless solid (6.30 g, $74 \%$ yield, 2 steps).

Melting Point: $\mathrm{mp} 276{ }^{\circ} \mathrm{C}$ (toluene). NMR Spectroscopy: ${ }^{1} \mathrm{H}$ NMR (300 MHz, DMSO-d-6) $\delta$ : 9.70 (bs, 1H), 7.66-7.65 (m, 2H), 7.64-7.61 (m, 4H), 4.64 (s, 4H). ${ }^{13} \mathrm{C}$ NMR (75 MHz, DMSOd-6) $\delta: 161.6,156.5$ (dd, $J=252.4 \mathrm{~Hz}, 3.6 \mathrm{~Hz}), 131.6$ (t, $J=10.0 \mathrm{~Hz}), 113.2-112.9$ (m), 66.4, 111.9-111.6 (m). ${ }^{19} \mathrm{~F}$ NMR (282 MHz, DMSO-d-6) $\delta:-119.2$. Mass Spectrometry HR-FAB NBA Calcd for $\mathrm{C}_{15} \mathrm{H}_{11} \mathrm{~N}_{2} \mathrm{~F}_{4}, 295.0858$. Found, 295.0858. Combustion Analysis: Anal. calcd for $\mathrm{C}_{15} \mathrm{H}_{11} \mathrm{~N}_{2} \mathrm{~F}_{5} \mathrm{C}, 54.36$; H, 3.35; N, 8.47; F, 22.98. Found C, 54.36; H, 3.61; N, 8.29.

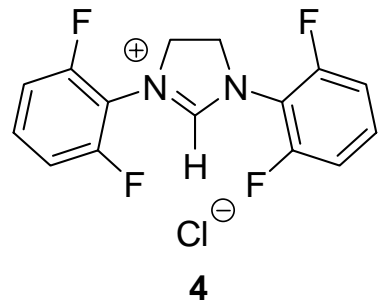

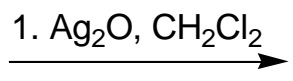

2. 1, toluene

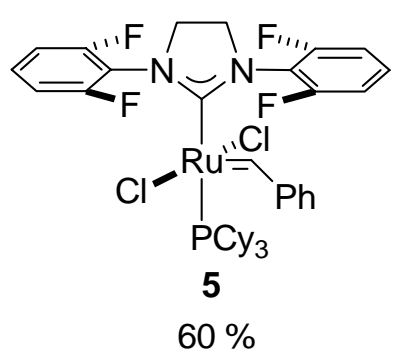

Fluoro-phosphine derived ruthenium complex 5. To tetrafluorodihydroimidazolium chloride 4 (661 mg, $2.00 \mathrm{mmol}, 1.00$ equiv), silver(I) oxide ( $255 \mathrm{mg}, 1.10 \mathrm{mmol}, 0.55$ equiv), and $4 \AA$ molecular sieves $(661 \mathrm{mg})$ in the dark in the glovebox at $25{ }^{\circ} \mathrm{C}$ was added $\mathrm{CH}_{2} \mathrm{Cl}_{2}(8.00 \mathrm{ml}$, c= $0.25 \mathrm{M}$ ) and the black suspension is vigorously stirred for $1 \mathrm{~h}$. The grey suspension was filtered through a small pad of celite. The filtrate was cooled to $-30{ }^{\circ} \mathrm{C}$ in a dark vial and the colorless crystals formed were filtered off $(450 \mathrm{mg}$ ). A portion of these colorless crystals (358 $\mathrm{mg}, 0.818$ mmol, 1.00 equiv) were placed in the dark in a vial. A solution of ruthenium complex 1 (673 $\mathrm{mg}, 0.818 \mathrm{mmol}, 1.00$ equiv) in toluene $(17.0 \mathrm{ml}, \mathrm{c}=0.05 \mathrm{M})$ was added and the resulting suspension was vigorously stirred at $20^{\circ} \mathrm{C}$ in the dark for $11 \mathrm{~h}$. The resulting suspension was poured onto a column packed with TSI Scientific silica gel and the complex was purified by chromatography eluting with pentane $/ \mathrm{Et}_{2} \mathrm{O}(4: 1,70 \mathrm{ml}),(1: 1,60 \mathrm{ml})$, and $(1: 4,200 \mathrm{ml})$. The dark red solution is concentrated in vacuo to afford a red powder ( $410 \mathrm{mg}, 60 \%$ yield). The complex is stable in air in the solid state, but not in solution, it is soluble in $\mathrm{CH}_{2} \mathrm{Cl}_{2}, \mathrm{CHCl}_{3}$, 
benzene, and toluene, but not in pentane. Crystals suitable for X-ray crystallography were grown by slow diffusion of pentane into solution of 5 in toluene at $25^{\circ} \mathrm{C}$.

NMR Spectroscopy: ${ }^{1} \mathrm{H}$ NMR (500 MHz, benzene-d6) $\delta: 20.19$ (s, 1H), 8.36 (bs, 1H), 7.24-7.21 (m, 1H), 7.08-7.02 (m, 4H), 6.77 (bs, 2H), 6.28 (bs, 1H), 6.08 (bs, 2H), 4.28 (s, 4H), 3.45-3.33 (m, 3H), 2.46-2.42 (m, 2H), 2.17-2.13 (m, 5H), 1.74-1.62 (m, 11H), 1.27-1.15 (m, 12H). ${ }^{13} \mathrm{C}$ NMR (125 MHz, benzene-d6, due to extensive fluorine coupling, coupling constants are not given, and resonances are reported as peaks) $\delta: 299.5-298.3(\mathrm{~m}), 224.3(\mathrm{~d}, 81 \mathrm{~Hz}), 163.6-158.8$ (m), 152.6-152.0 (m), 131.5-127.6 (m), 119.5-118.8 (m), 113.2-112.2 (m), 53.4, 52.8, 32.9, $32.4,32.3,31.9,30.4,30.0,29.4,29.0,28.4,28.0,27.9,27.0,26.6 .{ }^{19} \mathrm{~F}$ NMR (282 MHz,

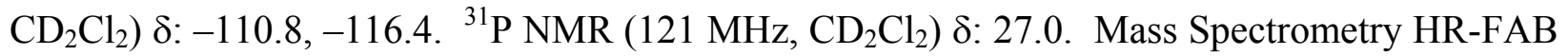
NBA Calcd for $\mathrm{C}_{40} \mathrm{H}_{49} \mathrm{~N}_{2} \mathrm{~F}_{4} \mathrm{Cl}_{2} \mathrm{PRu}, 836.1991$. Found, 836.1956.
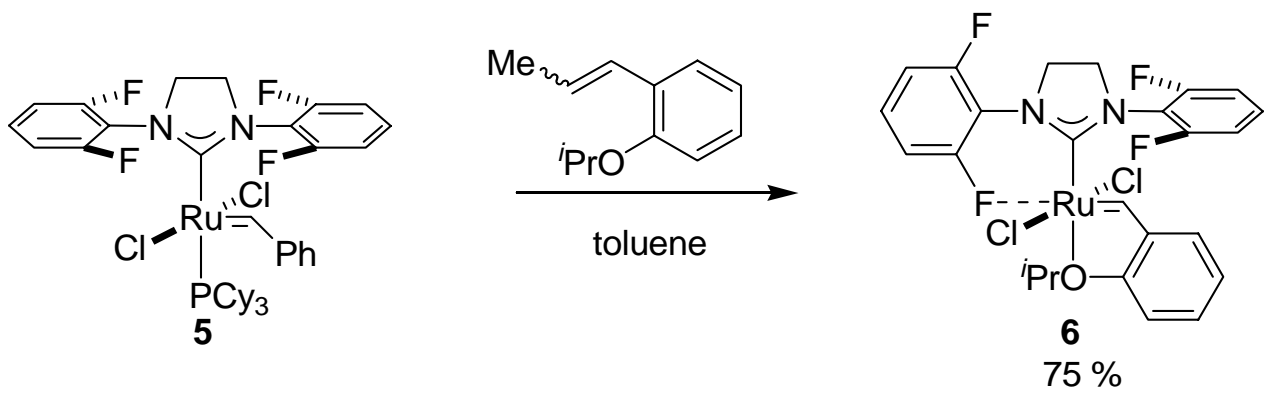

\section{Fluoro-ether derived ruthenium complex 6.}

To ruthenium benzylidene $5(137 \mathrm{mg}, 164 \mu \mathrm{mol}, 1.00$ equiv) in toluene $(8 \mathrm{ml})$ was added $o$ isopropoxy- $\beta$-methylstyrene ( $289 \mathrm{mg}, 1.64 \mathrm{mmol}, 10.0$ equiv). The dark red solution is left inside a capped vial without stirring. After $16 \mathrm{~h}$ at $23{ }^{\circ} \mathrm{C}$ the liquid is decanted off, leaving behind crystalline 6 . The crystals are washed with toluene $(2 \times 2 \mathrm{ml})$ and diethylether $(2 \times 2 \mathrm{ml})$ and dried to afford dark brown crystals (76 mg, $75 \%$ yield). The complex is stable in air for extended periods of time in the solid state and in solution; it is soluble in $\mathrm{CH}_{2} \mathrm{Cl}_{2}$ and $\mathrm{CHCl}_{3}$, but not in benzene, toluene, diethylether, acetone, and pentane. Crystals suitable for X-ray crystallography were grown by slow diffusion of pentane into solution of 6 in $\mathrm{CH}_{2} \mathrm{Cl}_{2}$ at $25{ }^{\circ} \mathrm{C}$.

NMR Spectroscopy: ${ }^{1} \mathrm{H}$ NMR (500 MHz, $\left.\mathrm{CD}_{2} \mathrm{Cl}_{2}\right) \delta: 17.14(\mathrm{~s}, 1 \mathrm{H}), 7.60-7.57(\mathrm{~m}, 1 \mathrm{H}), 7.43-$ $7.37(\mathrm{~m}, 2 \mathrm{H}), 7.26-7.25(\mathrm{~m}, 1 \mathrm{H}), 7.11-7.06(\mathrm{~m}, 4 \mathrm{H}), 6.99-6.96(\mathrm{~m}, 2 \mathrm{H}), 5.07$ (qn, $J=6.2 \mathrm{~Hz}$, $1 \mathrm{H}), 4.31(\mathrm{~s}, 4 \mathrm{H}), 1.52(\mathrm{~d}, J=6.2 \mathrm{~Hz}, 6 \mathrm{H}) .{ }^{13} \mathrm{C} \mathrm{NMR}\left(125 \mathrm{MHz}, \mathrm{CD}_{2} \mathrm{Cl}_{2}\right.$, due to extensive fluorine coupling, coupling constants are not given, and resonances are reported as peaks) $\delta$ : 289.5, 215.9, 161.6, 161.6, 159.6, 159.6, 153.8, 144.6, 130.7, 130.4, 130.3, 130.2, 129.5, 128.7, 123.0, 122.6, 120.4, 113.8, 112.8, 112.7, 112.6, 75.9, 53.1, 21.9. ${ }^{19} \mathrm{~F}$ NMR $\left(471 \mathrm{MHz}, \mathrm{CD}_{2} \mathrm{Cl}_{2}\right)$ 
$\delta$ : -114.2. Mass Spectrometry HR-FAB NBA Calcd for $\mathrm{C}_{25} \mathrm{H}_{22} \mathrm{~N}_{2} \mathrm{~F}_{4} \mathrm{Cl}_{2} \mathrm{ORu}$, 614.0089. Found, 614.0112 .

The chloro-analogs S3 and S6 were prepared as well. We believe that the reduced stability of S3 and $\mathbf{S 6}$ compared to $\mathbf{5}$ and $\mathbf{6}$, respectively, results from an activation of the $\mathrm{C}-\mathrm{Cl}$ bond of the NHC ligand, based on the isolation of a decomposition product, in which oxidative addition of the $\mathrm{C}-\mathrm{Cl}$ bond to the ruthenium was observed, identified by a low quality $\mathrm{x}$-ray structure, in an attempt to obtain a crystal structure of S3. Complexes S3 and S6 are less efficient olefin metathesis catalysts compared to their fluorinated counterparts, presumably due to their instability.<smiles>O=C(Nc1c(Cl)cccc1Cl)C(=O)Nc1c(Cl)cccc1Cl</smiles>

S2

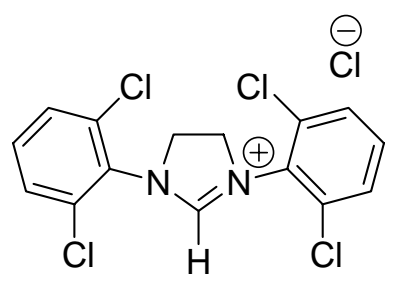

S4

\section{Tetrachloro diamide S2.}

The same procedure as described for $\mathbf{S 1}$ was employed to afford the product as a colorless solid in $61 \%$ yield.

Melting Point: mp $275{ }^{\circ} \mathrm{C}$ (toluene). NMR Spectroscopy: ${ }^{1} \mathrm{H}$ NMR (300 MHz, DMSO-d-6) $\delta$ : $10.88(\mathrm{~s}, 2 \mathrm{H}), 7.62-7.59(\mathrm{~m}, 4 \mathrm{H}), 7.42(\mathrm{dd}, J=7.7,7.5 \mathrm{~Hz}, 2 \mathrm{H}) .{ }^{13} \mathrm{C}$ NMR $(75 \mathrm{MHz}$, DMSO-d6) $\delta: 158.3,133.6,132.1,129.9,128.6$. Mass Spectrometry HR-FAB NBA Calcd for $\mathrm{C}_{14} \mathrm{H}_{9} \mathrm{~N}_{2} \mathrm{O}_{2} \mathrm{Cl}_{4}$, 376.9418. Found, 376.9403. Combustion Analysis: Anal. calcd for $\mathrm{C}_{14} \mathrm{H}_{9} \mathrm{~N}_{2} \mathrm{O}_{2} \mathrm{Cl}_{4}$ : C, 44.48; H, 2.13; N, 7.41; Cl, 37.51. Found C, 44.49; H, 2.25; N, 7.19.

\section{Tetrachlorodihydroimidazolium chloride S4.}

The same procedure as described for $\mathbf{4}$ was employed to afford the product as a colorless solid in $82 \%$ yield.

Melting Point: $\mathrm{mp}>300{ }^{\circ} \mathrm{C}$ (toluene). NMR Spectroscopy: ${ }^{1} \mathrm{H}$ NMR (300 MHz, DMSO-d-6) $\delta$ : $9.91(\mathrm{~s}, 1 \mathrm{H}), 7.82-7.79(\mathrm{~m}, 4 \mathrm{H}), 7.67(\mathrm{dd}, J=9.0,7.4 \mathrm{~Hz}, 2 \mathrm{H}), 4.62(\mathrm{~s}, 4 \mathrm{H}) .{ }^{13} \mathrm{C} \mathrm{NMR}(75 \mathrm{MHz}$, DMSO-d-6) $\delta: 162.8,133.2,133.0,130.1,129.6$, 51.3. Mass Spectrometry HR-FAB NBA Calcd for $\mathrm{C}_{15} \mathrm{H}_{11} \mathrm{~N}_{2} \mathrm{Cl}_{4}, 358.9676$. Found, 358.9680. Combustion Analysis: Anal. calcd for $\mathrm{C}_{15} \mathrm{H}_{11} \mathrm{~N}_{2} \mathrm{Cl}_{5} \mathrm{C}, 45.43 ; \mathrm{H}, 2.80 ; \mathrm{N}, 7.06 ; \mathrm{Cl}, 44.70$. Found C, 45.16; H, 2.96; N, 6.77. 


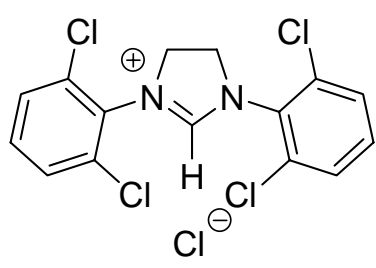

S4

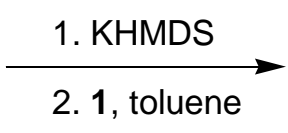

2. 1, toluene

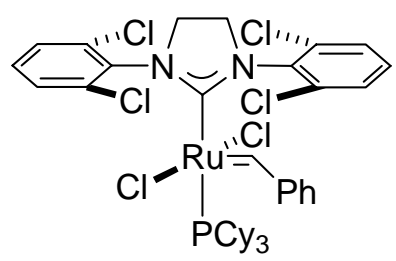

S3

$31 \%$

\section{Chloro-phosphine derived ruthenium complex S3}

To S4 (78 mg, $0.20 \mathrm{mmol}, 2.0$ equiv) and KHMDS (40 mg, $0.20 \mathrm{mmol}, 2.0$ equiv) was added toluene $(1.0 \mathrm{ml})$ at $20{ }^{\circ} \mathrm{C}$. The suspension is stirred for $15 \mathrm{~min}$. To this suspension is added 1 $(82 \mathrm{mg}, 0.10 \mathrm{mmol}, 1.0$ equiv) in toluene $(1.0 \mathrm{ml})$ and the resulting suspension is stirred for $14 \mathrm{~h}$. The resulting suspension was poured onto a column packed with TSI Scientific silica gel and the complex was purified by chromatography eluting with pentane/Et $2 \mathrm{O}(4: 1 \rightarrow 1: 1)$. The dark red solution is concentrated in vacuo to afford a red powder (28 mg, $31 \%$ yield). The complex is stable in air in the solid state, but not in solution, it is soluble in $\mathrm{CH}_{2} \mathrm{Cl}_{2}, \mathrm{CHCl}_{3}$, benzene, and toluene, but not in pentane.

NMR Spectroscopy: ${ }^{1} \mathrm{H}$ NMR (300 MHz, $\left.\mathrm{CDCl}_{3}\right) \delta$ : $19.72(\mathrm{~s}, 1 \mathrm{H}), 7.59-6.58(\mathrm{~m}, 11 \mathrm{H}), 4.13$ (bs, 4H), 2.18-1.02 (m, 33H). ${ }^{31} \mathrm{P}$ NMR (121 MHz, $\left.\mathrm{CDCl}_{3}\right) \delta:$ 29.5. Mass Spectrometry HR-FAB NBA Calcd for $\mathrm{C}_{40} \mathrm{H}_{49} \mathrm{~N}_{2} \mathrm{Cl}_{6} \mathrm{PRu}$, 902.0789. Found, 902.0762.

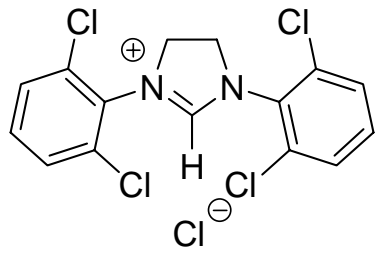

S4

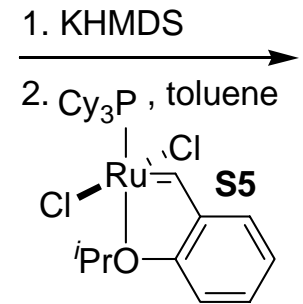

3. $\mathrm{CuCl}$

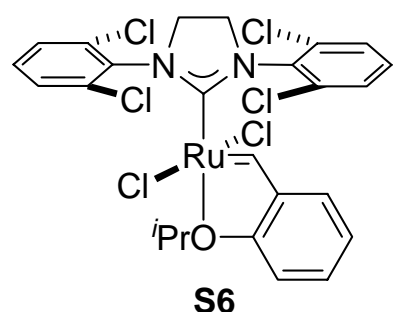

$31 \%$

\section{Chloro-ether derived ruthenium complex S6}

To S4 (397 mg, $1.00 \mathrm{mmol}, 2.00$ equiv) and KHMDS (199 mg, $1.00 \mathrm{mmol}, 2.00$ equiv) was added toluene $(5.0 \mathrm{ml})$ at $20{ }^{\circ} \mathrm{C}$. The suspension is stirred for $15 \mathrm{~min}$. To this suspension is added $\mathrm{S5}^{2}$ (300 $\mathrm{mg}, 0.500 \mathrm{mmol}, 1.00$ equiv) in toluene $(5.0 \mathrm{ml})$ and the resulting suspension is

2 Harrity, J. P. A.; La, D. S.; Cefalo, D. R.; Visser, M. S.; Hoveyda, A. H. J. Am. Chem. Soc. 1998, 120, 2343-2351. (b) Kingsbury, J. S.; Harrity, J. P. A.; Bonitatebus, P. J.; Hoveyda, A. H. J. Am. Chem. Soc. 1999, 121, 791-799. 
stirred for $2 \mathrm{~h}$. To this suspension is added $\mathrm{Cu}(\mathrm{I}) \mathrm{Cl}(51.0 \mathrm{mg}, 0.500 \mathrm{mmol}, 1.00$ equiv) and the suspension is stirred for $1 \mathrm{~h}$. The resulting suspension was poured onto a column packed with TSI Scientific silica gel and the complex was purified by chromatography eluting with $\mathrm{CH}_{2} \mathrm{Cl}_{2}$. The dark green-brown solution is concentrated in vacuo to afford a green powder (150 mg, $44 \%$ yield). The complex is stable in air in the solid state; it is soluble in $\mathrm{CH}_{2} \mathrm{Cl}_{2}, \mathrm{CHCl}_{3}$, benzene, but not in pentane. Crystals suitable for $\mathrm{X}$-ray crystallography were grown by slow diffusion of pentane into solution of $\mathbf{S 6}$ in $\mathrm{CH}_{2} \mathrm{Cl}_{2}$ at $25^{\circ} \mathrm{C}$.

NMR Spectroscopy: ${ }^{1} \mathrm{H}$ NMR (300 MHz, $\left.\mathrm{CDCl}_{3}\right) \delta: 17.24(\mathrm{~s}, 1 \mathrm{H}), 7.49-7.46(\mathrm{~m}, 5 \mathrm{H}), 7.32(\mathrm{dd}$, $J=8.5,7.1 \mathrm{~Hz}, 2 \mathrm{H}), 7.17(\mathrm{dd}, J=7.7,1.6 \mathrm{~Hz}, 1 \mathrm{H}), 6.89$ (dd, $J=7.7,6.9 \mathrm{~Hz}, 1 \mathrm{H}), 5.01(\mathrm{sep}, J=$ $6.0 \mathrm{~Hz}, 1 \mathrm{H}), 4.32(\mathrm{~s}, 4 \mathrm{H}), 1.54(\mathrm{~d}, J=6.0 \mathrm{~Hz}, 6 \mathrm{H})$. Mass Spectrometry HR-FAB NBA Calcd for $\mathrm{C}_{25} \mathrm{H}_{22} \mathrm{~N}_{2} \mathrm{Cl}_{6} \mathrm{ORu}, 679.8917$. Found, 679.8909.

Upon attempted crystallization of the complex, a decomposition product could be isolated. Its low quality crystal structure suggested $\mathrm{C}-\mathrm{F}$ activation pathway for catalyst decomposition.
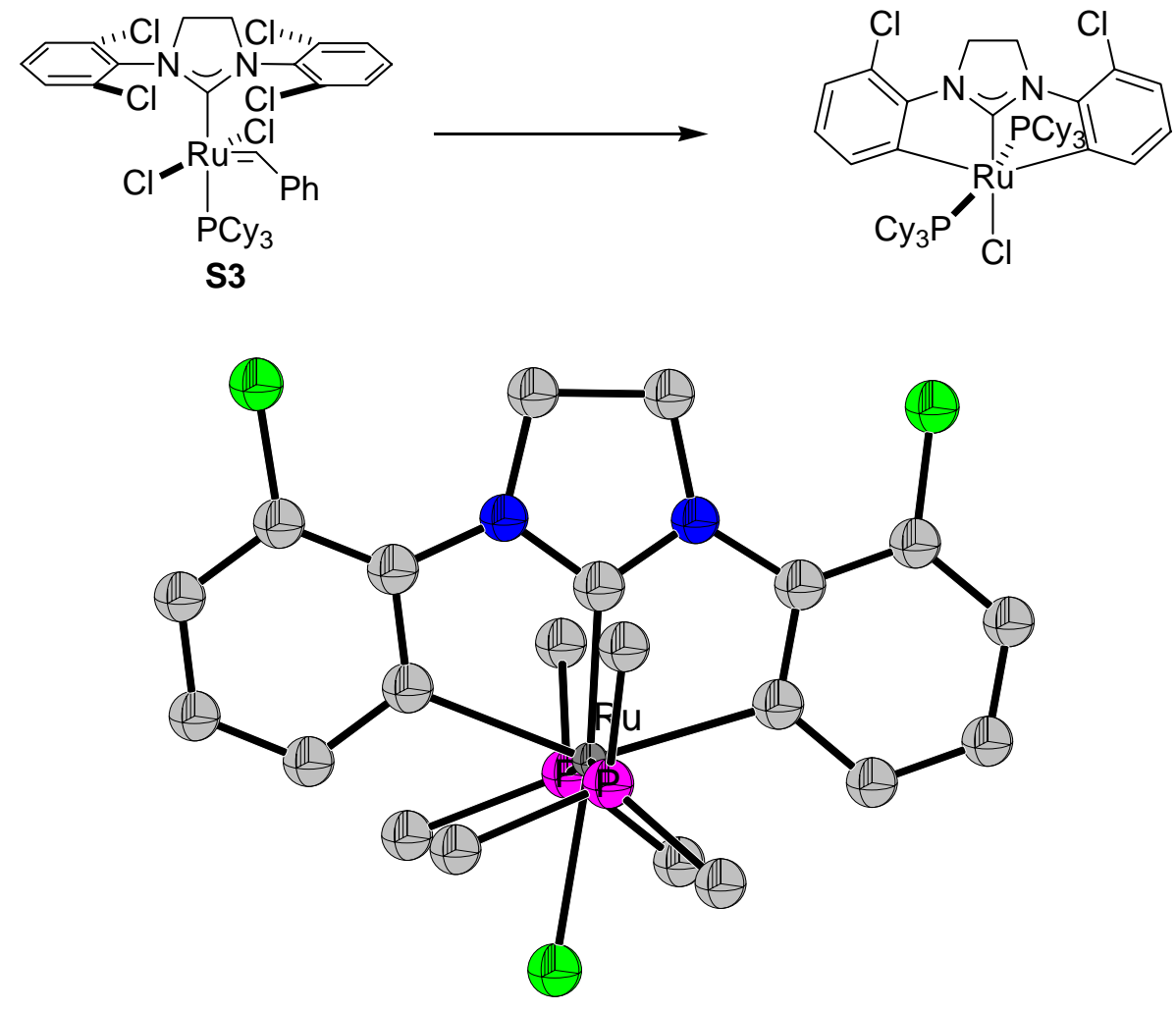


\section{Crystallographic Analysis}

Difluoro-phosphine derived ruthenium complex 5

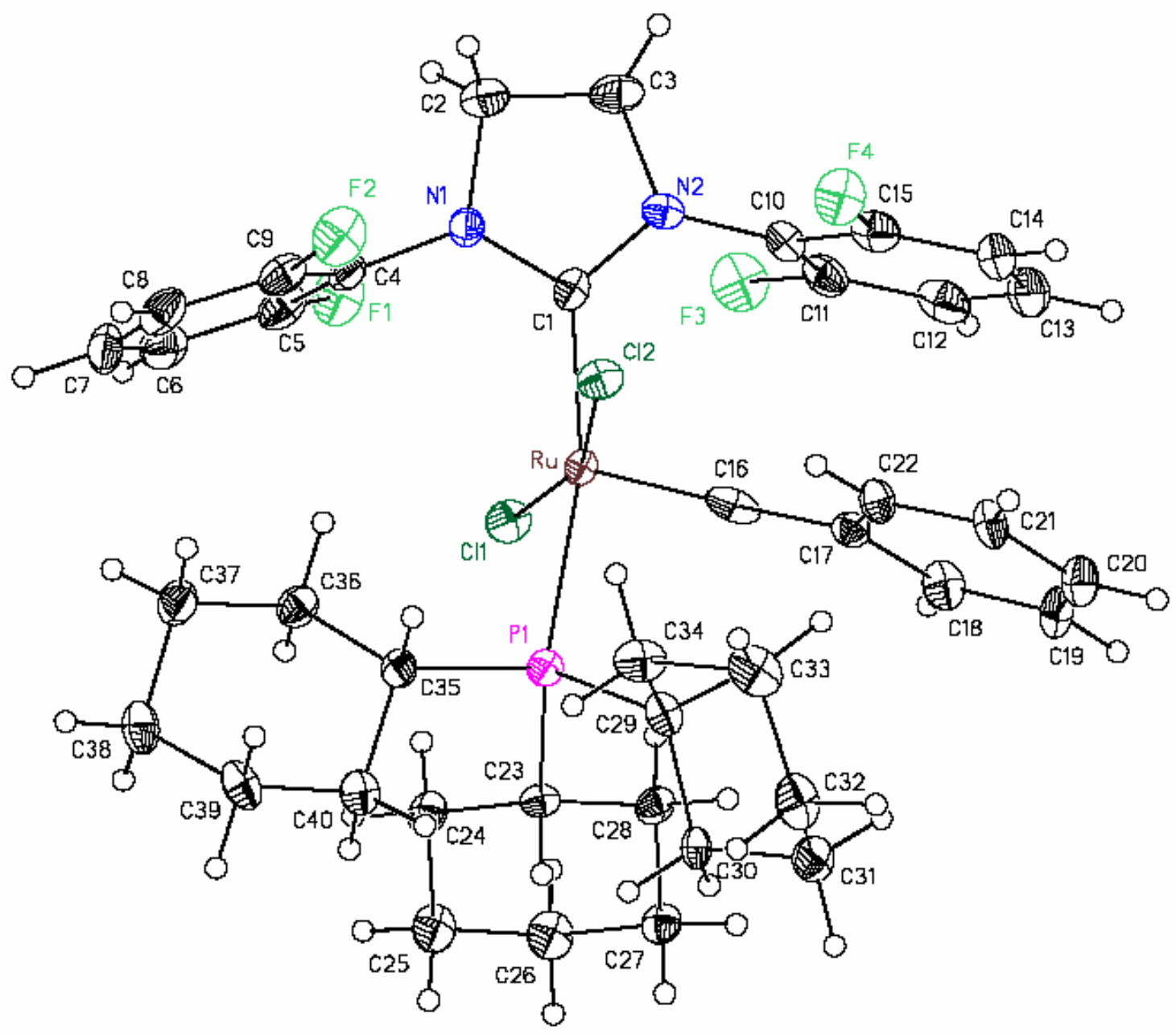


Table 1. Crystal data and structure refinement for CCDC 279454.

Empirical formula

Formula weight

Crystallization Solvent

Crystal Habit

Crystal size

Crystal color

ype of diffractometer

Wavelength

Data Collection Temperature

$\theta$ range for 7845 reflections used

in lattice determination

Unit cell dimensions

Volume

Z

Crystal system

Space group

Density (calculated)

$\mathrm{F}(000)$

Data collection program

$\theta$ range for data collection
$\mathrm{C}_{40} \mathrm{H}_{49} \mathrm{~F}_{4} \mathrm{~N}_{2} \mathrm{PCl}_{2} \mathrm{Ru}$

836.75

THF/diethylether

Needle

$0.24 \times 0.10 \times 0.09 \mathrm{~mm}^{3}$

Dichroic - red/green

\section{Data Collection}

Bruker SMART 1000

$0.71073 \AA \mathrm{MoK} \alpha$

100(2) K

2.17 to $28.06^{\circ}$

$\mathrm{a}=9.7554(13) \AA$

$\alpha=87.666(2)^{\circ}$

$\mathrm{b}=10.0375(14) \AA$

$\beta=77.183(2)^{\circ}$

$\mathrm{c}=19.968(3) \AA$

$\gamma=85.699(2)^{\circ}$

$1900.6(5) \AA^{3}$

2

Triclinic

P-1

$1.462 \mathrm{Mg} / \mathrm{m}^{3}$

864

Bruker SMART v5.630

2.04 to $28.49^{\circ}$ 
Completeness to $\theta=28.49^{\circ}$

Index ranges

Data collection scan type

Data reduction program

Reflections collected

Independent reflections

Absorption coefficient

Absorption correction

Max. and min. transmission
$91.0 \%$

$-13 \leq \mathrm{h} \leq 12,-13 \leq \mathrm{k} \leq 13,-26 \leq 1 \leq 25$

$\omega$ scans at $5 \phi$ settings

Bruker SAINT v6.45A

27431

$8763\left[\mathrm{R}_{\mathrm{int}}=0.1408\right]$

$0.646 \mathrm{~mm}^{-1}$

None

0.9441 and 0.8603 


\section{Table 1 (cont.)}

\section{Structure solution and Refinement}

\begin{tabular}{|c|c|}
\hline Structure solution program & Bruker XS v6.12 \\
\hline Primary solution method & Direct methods \\
\hline Secondary solution method & Difference Fourier map \\
\hline Hydrogen placement & Geometric positions \\
\hline Structure refinement program & Bruker XL v6.12 \\
\hline Refinement method & Full matrix least-squares on $\mathrm{F}^{2}$ \\
\hline Data / restraints / parameters & 8763 / 0 / 451 \\
\hline Treatment of hydrogen atoms & Riding \\
\hline Goodness-of-fit on $\mathrm{F}^{2}$ & 1.320 \\
\hline Final $\mathrm{R}$ indices $[\mathrm{I}>2 \sigma(\mathrm{I}), 5887$ reflections $]$ & $\mathrm{R} 1=0.0642, w \mathrm{R} 2=0.1203$ \\
\hline $\mathrm{R}$ indices (all data) & $\mathrm{R} 1=0.1046, w \mathrm{R} 2=0.1289$ \\
\hline Type of weighting scheme used & Sigma \\
\hline Weighting scheme used & $w=1 / \sigma^{2}\left(\mathrm{Fo}^{2}\right)$ \\
\hline Max shift/error & 0.001 \\
\hline Average shift/error & 0.000 \\
\hline Largest diff. peak and hole & 1.925 and -1.244 e. $\AA^{-3}$ \\
\hline
\end{tabular}

\section{Special Refinement Details}

In this structure the 2,6-difluorophenyl rings show no significant twisting with respect to the imidazole ring, Figure 4 and Table 6 . Additionally, the entire 1,3-bis(2,6-difluorophenyl)-4,5-dihydroimidazole ligand does not show significant rotation around the $\mathrm{C}(1)-\mathrm{Ru}$ bond, Figure 5 and Table 6 . The sterics of the tricyclohexalphoshine ligand may explain the conformation seen here, contrasted with the conformations previously reported in structures labeled as TAR04, TAR05 and TAR07.

Refinement of $\mathrm{F}^{2}$ against ALL reflections. The weighted R-factor $(w \mathrm{R})$ and goodness of fit $(\mathrm{S})$ are based on $\mathrm{F}^{2}$, conventional R-factors (R) are based on $\mathrm{F}$, with $\mathrm{F}$ set to zero for negative $\mathrm{F}^{2}$. The threshold expression of $\mathrm{F}^{2}$ $>2 \sigma\left(\mathrm{F}^{2}\right)$ is used only for calculating R-factors $(\mathrm{gt})$ etc. and is not relevant to the choice of reflections for 
refinement. R-factors based on $\mathrm{F}^{2}$ are statistically about twice as large as those based on F, and R-factors based on ALL data will be even larger.

All esds (except the esd in the dihedral angle between two 1.s. planes) are estimated using the full covariance matrix. The cell esds are taken into account individually in the estimation of esds in distances, angles and torsion angles; correlations between esds in cell parameters are only used when they are defined by crystal symmetry. An approximate (isotropic) treatment of cell esds is used for estimating esds involving l.s. planes. 


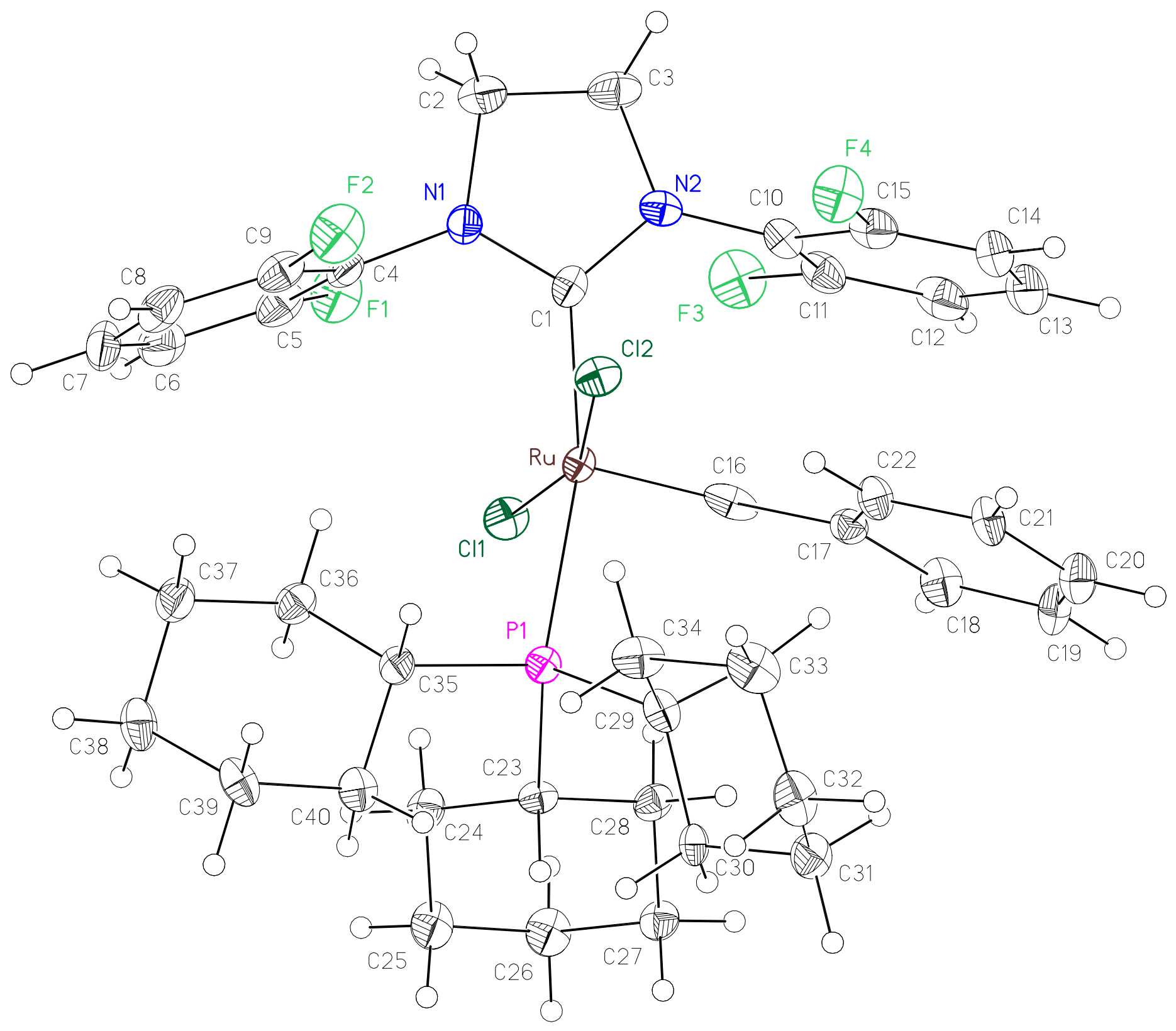




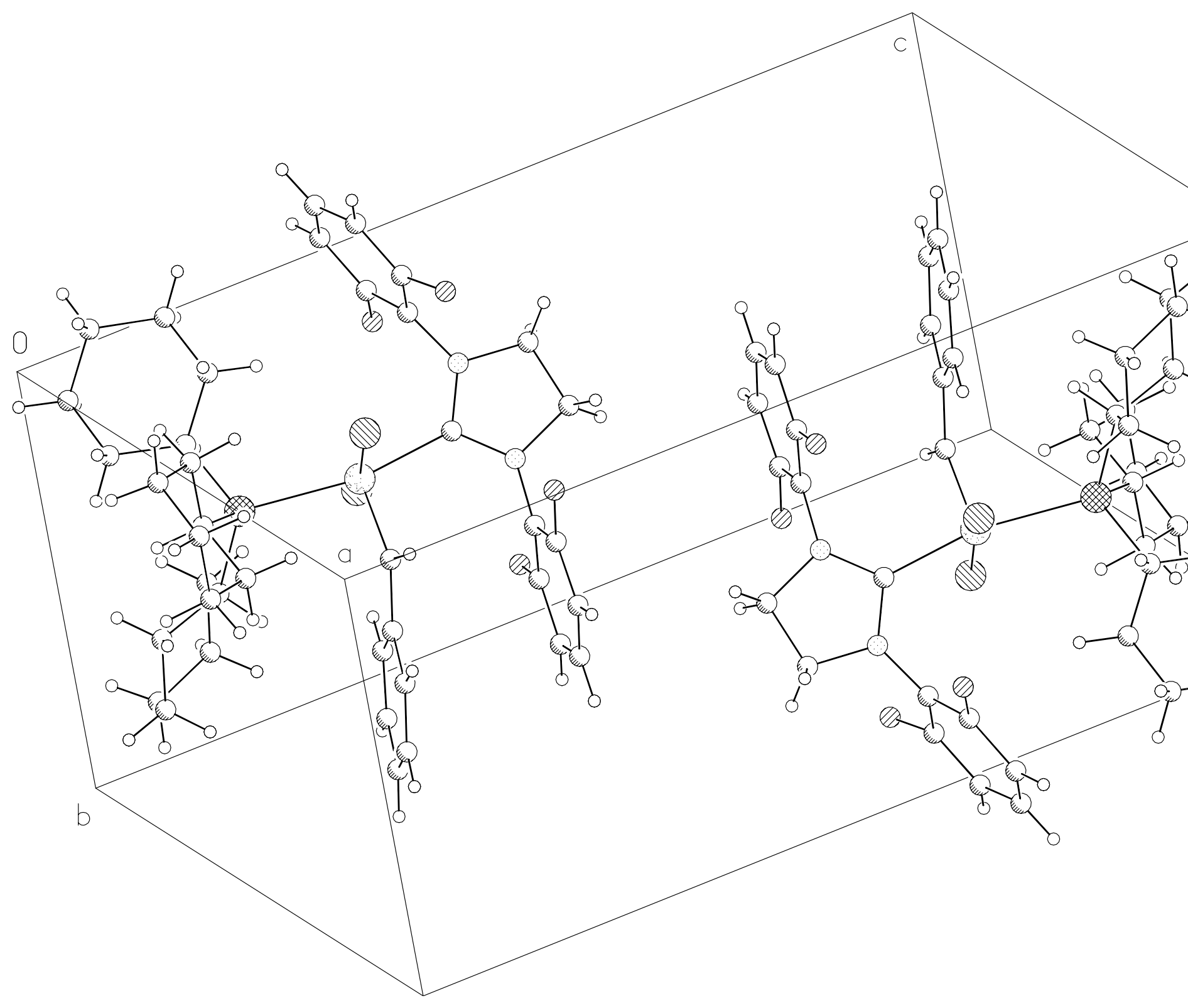



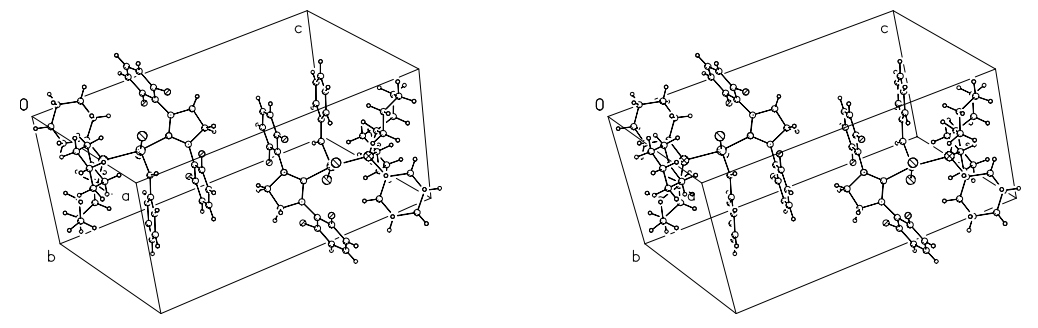


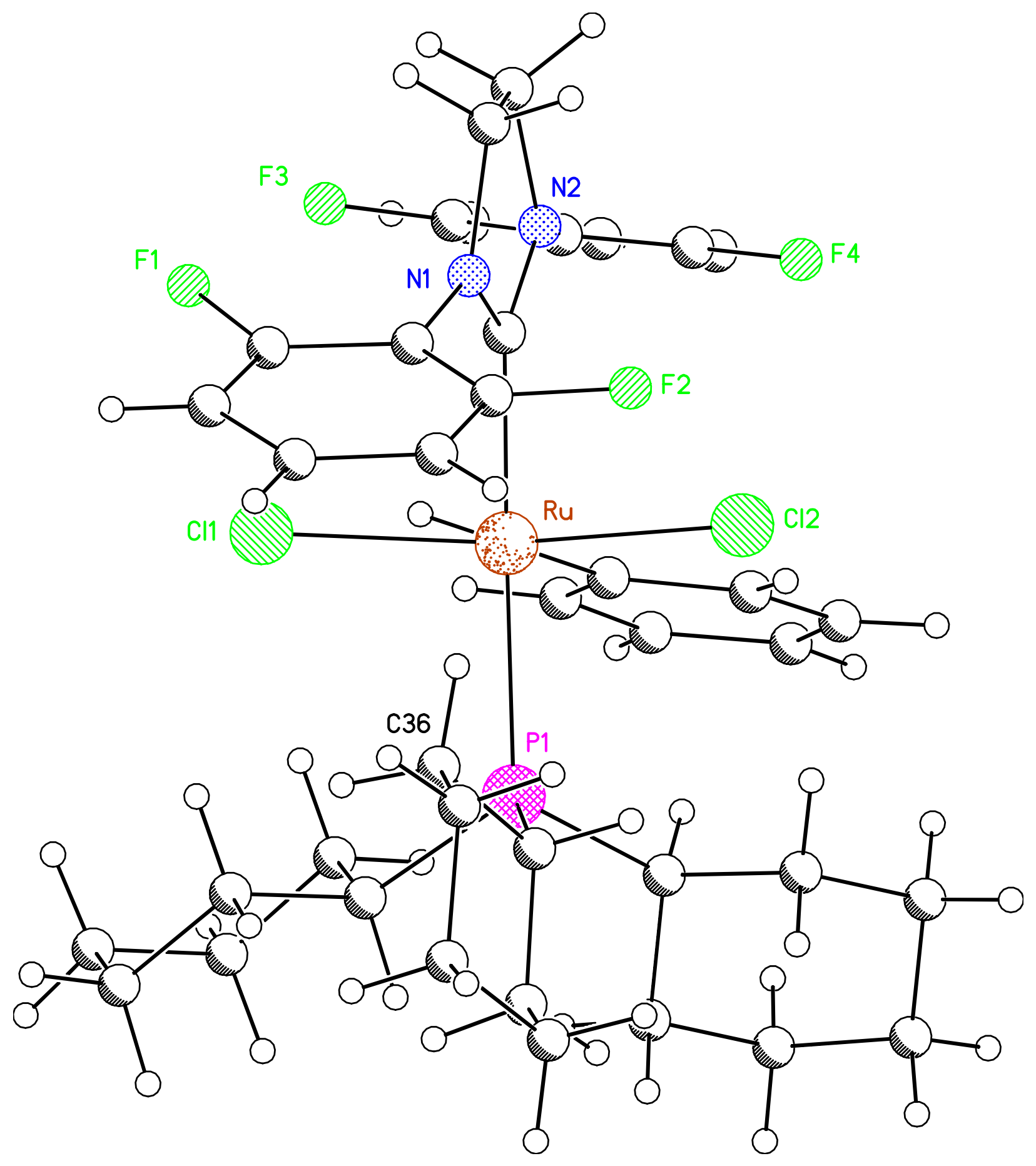




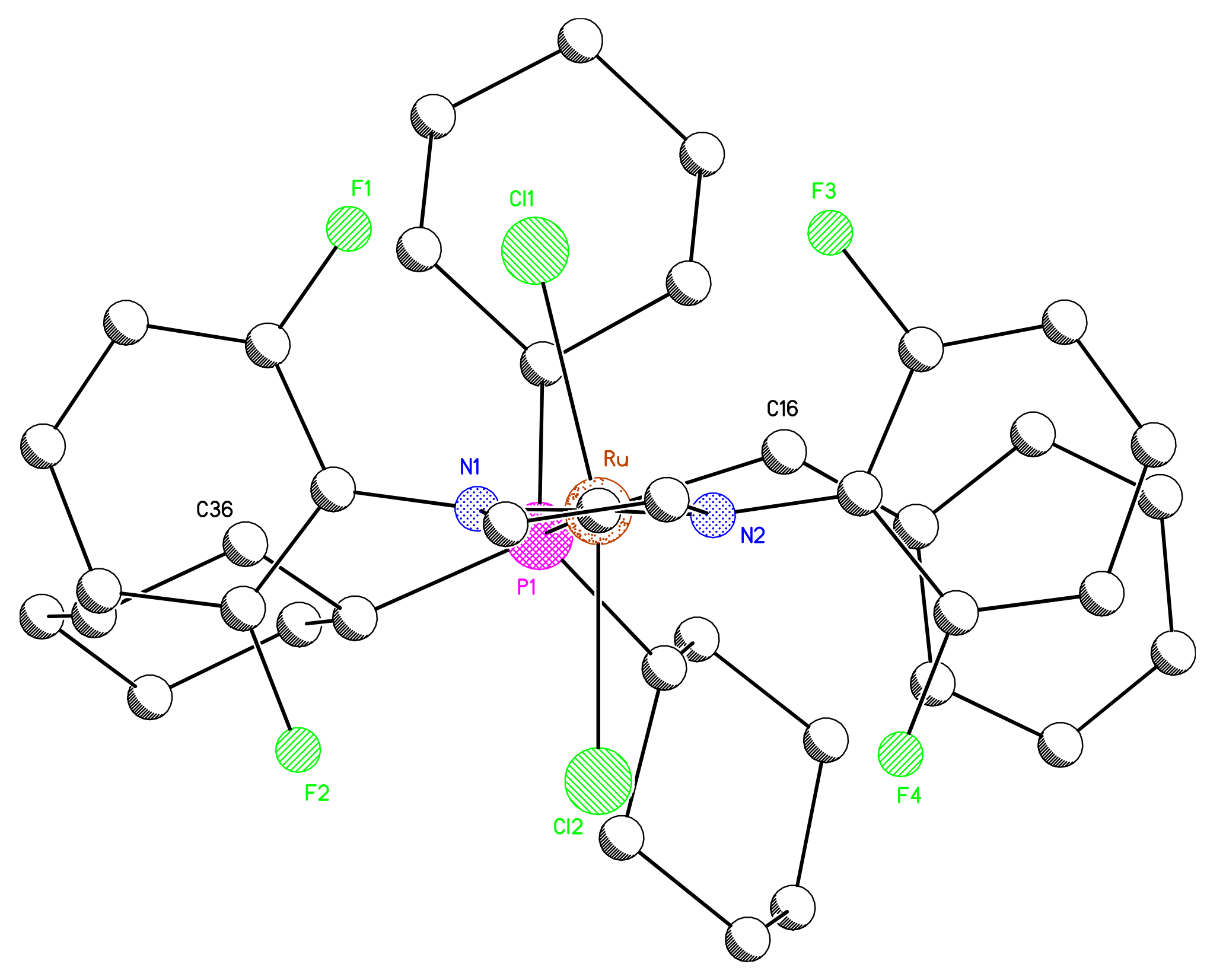


Table 2. Atomic coordinates $\left(x 1^{4}\right)$ and equivalent isotropic displacement parameters $\left(\AA^{2} \times 1^{3}\right)$ for CCDC 279454. U(eq) is defined as the trace of the orthogonalized Uij tensor.

\begin{tabular}{|c|c|c|c|c|}
\hline & $\mathrm{x}$ & $\mathrm{y}$ & $\mathrm{z}$ & $\mathrm{U}_{\text {eq }}$ \\
\hline $\mathrm{Ru}$ & $8321(1)$ & $5811(1)$ & $7154(1)$ & $13(1)$ \\
\hline $\mathrm{Cl}(1)$ & $6930(1)$ & $7836(1)$ & $7426(1)$ & $22(1)$ \\
\hline $\mathrm{Cl}(2)$ & 10160(1) & 4193(1) & $6656(1)$ & $20(1)$ \\
\hline $\mathrm{P}(1)$ & $9037(1)$ & $5620(1)$ & $8251(1)$ & $15(1)$ \\
\hline $\mathrm{F}(1)$ & 7799(3) & $9625(3)$ & $6053(2)$ & $31(1)$ \\
\hline $\mathrm{F}(2)$ & $11764(3)$ & $6605(3)$ & $5685(2)$ & $33(1)$ \\
\hline $\mathrm{F}(3)$ & 4707(3) & $6553(3)$ & $6240(2)$ & $32(1)$ \\
\hline $\mathrm{F}(4)$ & $7875(3)$ & $2805(3)$ & $5796(2)$ & $29(1)$ \\
\hline $\mathrm{N}(1)$ & $8896(4)$ & $7082(4)$ & $5734(2)$ & $19(1)$ \\
\hline $\mathrm{N}(2)$ & $7407(4)$ & $5564(4)$ & $5775(2)$ & $17(1)$ \\
\hline$C(1)$ & $8172(5)$ & $6170(4)$ & $6151(2)$ & $16(1)$ \\
\hline$C(2)$ & $8719(6)$ & $7058(6)$ & $5020(3)$ & $30(1)$ \\
\hline$C(3)$ & $7502(6)$ & $6200(5)$ & $5086(3)$ & $26(1)$ \\
\hline$C(4)$ & $9724(5)$ & $8039(5)$ & $5912(2)$ & $19(1)$ \\
\hline$C(5)$ & $9154(5)$ & $9346(5)$ & $6073(3)$ & $25(1)$ \\
\hline$C(6)$ & $9940(6)$ & $10324(5)$ & $6213(3)$ & $29(1)$ \\
\hline$C(7)$ & $11351(6)$ & $10027(5)$ & $6179(3)$ & $28(1)$ \\
\hline $\mathrm{C}(8)$ & $11983(6)$ & 8783(6) & $6000(3)$ & $27(1)$ \\
\hline$C(9)$ & $11149(5)$ & $7819(5)$ & $5870(2)$ & $23(1)$ \\
\hline$C(10)$ & $6329(5)$ & $4711(5)$ & $6026(2)$ & $18(1)$ \\
\hline$C(11)$ & $4940(5)$ & $5211(5)$ & $6252(3)$ & $23(1)$ \\
\hline$C(12)$ & $3843(5)$ & $4423(6)$ & $6478(3)$ & $28(1)$ \\
\hline$C(13)$ & $4130(6)$ & $3056(6)$ & $6474(3)$ & $33(1)$ \\
\hline$C(14)$ & $5487(6)$ & $2470(6)$ & $6244(3)$ & $28(1)$ \\
\hline$C(15)$ & $6544(5)$ & $3323(5)$ & $6021(3)$ & $23(1)$ \\
\hline$C(16)$ & $6732(5)$ & $4941(5)$ & $7470(2)$ & $20(1)$ \\
\hline
\end{tabular}




\begin{tabular}{|c|c|c|c|c|}
\hline$C(17)$ & $6428(5)$ & $3553(5)$ & $7680(2)$ & $17(1)$ \\
\hline$C(18)$ & $5065(5)$ & $3307(5)$ & $8060(3)$ & $24(1)$ \\
\hline$C(19)$ & $4708(5)$ & $2027(5)$ & $8282(3)$ & $26(1)$ \\
\hline$C(20)$ & $5700(5)$ & $965(5)$ & $8114(3)$ & $23(1)$ \\
\hline $\mathrm{C}(21)$ & $7035(5)$ & $1190(5)$ & $7727(3)$ & $23(1)$ \\
\hline$C(22)$ & $7408(5)$ & $2459(5)$ & $7527(2)$ & $19(1)$ \\
\hline$C(23)$ & $7903(5)$ & 6475(4) & $9001(2)$ & $14(1)$ \\
\hline$C(24)$ & $7901(5)$ & $8002(5)$ & $9006(2)$ & $21(1)$ \\
\hline$C(25)$ & $6961(5)$ & $8562(5)$ & $9663(3)$ & $24(1)$ \\
\hline$C(26)$ & $5479(5)$ & $8115(5)$ & $9784(3)$ & $24(1)$ \\
\hline$C(27)$ & $5489(5)$ & $6602(5)$ & $9784(2)$ & $19(1)$ \\
\hline$C(28)$ & $6392(5)$ & $6070(5)$ & $9110(2)$ & $18(1)$ \\
\hline$C(29)$ & $9198(5)$ & $3852(4)$ & $8577(2)$ & $16(1)$ \\
\hline$C(30)$ & $8891(5)$ & $3562(4)$ & $9352(2)$ & $16(1)$ \\
\hline $\mathrm{C}(31)$ & $8780(6)$ & $2047(5)$ & $9484(3)$ & $25(1)$ \\
\hline$C(32)$ & $10131(5)$ & $1260(5)$ & $9141(3)$ & $26(1)$ \\
\hline$C(33)$ & $10544(6)$ & 1599(5) & $8380(3)$ & $29(1)$ \\
\hline$C(34)$ & $10634(5)$ & $3102(5)$ & $8252(3)$ & $24(1)$ \\
\hline$C(35)$ & $10807(5)$ & $6249(5)$ & $8152(2)$ & $18(1)$ \\
\hline$C(36)$ & $10941(5)$ & $7565(5)$ & $7743(2)$ & $21(1)$ \\
\hline$C(37)$ & $12419(5)$ & $8066(5)$ & $7636(3)$ & $26(1)$ \\
\hline$C(38)$ & $12863(5)$ & $8141(5)$ & $8311(3)$ & $25(1)$ \\
\hline $\mathrm{C}(39)$ & $12782(5)$ & $6798(5)$ & $8694(3)$ & $22(1)$ \\
\hline$C(40)$ & $11309(5)$ & $6314(5)$ & $8819(2)$ & $21(1)$ \\
\hline
\end{tabular}


Table 3. Selected bond lengths $[\AA]]$ and angles $\left[^{\circ}\right]$ for CCDC 279454.

\begin{tabular}{lccc}
\hline $\mathrm{Ru}-\mathrm{C}(16)$ & $1.814(5)$ & $\mathrm{C}(16)-\mathrm{Ru}-\mathrm{C}(1)$ & $99.8(2)$ \\
$\mathrm{Ru}-\mathrm{C}(1)$ & $2.055(5)$ & $\mathrm{C}(16)-\mathrm{Ru}-\mathrm{Cl}(1)$ & $87.63(14)$ \\
$\mathrm{Ru}-\mathrm{Cl}(1)$ & $2.3698(12)$ & $\mathrm{C}(1)-\mathrm{Ru}-\mathrm{Cl}(1)$ & $87.52(12)$ \\
$\mathrm{Ru}-\mathrm{Cl}(2)$ & $2.3976(11)$ & $\mathrm{C}(16)-\mathrm{Ru}-\mathrm{Cl}(2)$ & $107.82(14)$ \\
$\mathrm{Ru}-\mathrm{P}(1)$ & $2.4386(14)$ & $\mathrm{C}(1)-\mathrm{Ru}-\mathrm{Cl}(2)$ & $83.44(12)$ \\
& & $\mathrm{Cl}(1)-\mathrm{Ru}-\mathrm{Cl}(2)$ & $163.19(4)$ \\
& & $\mathrm{C}(16)-\mathrm{Ru}-\mathrm{P}(1)$ & $93.83(15)$ \\
& & $\mathrm{C}(1)-\mathrm{Ru}-\mathrm{P}(1)$ & $166.32(13)$ \\
& & $\mathrm{Cl}(1)-\mathrm{Ru}-\mathrm{P}(1)$ & $94.17(4)$ \\
& & $\mathrm{Cl}(2)-\mathrm{Ru}-\mathrm{P}(1)$ & $91.40(4)$ \\
\hline
\end{tabular}


Table 4. Bond lengths $[\AA \AA]$ and angles $\left[^{\circ}\right]$ for CCDC 279454.

\begin{tabular}{|c|c|c|c|}
\hline Ru-C(16) & $1.814(5)$ & $\mathrm{C}(11)-\mathrm{C}(12)$ & $1.363(7)$ \\
\hline $\mathrm{Ru}-\mathrm{C}(1)$ & $2.055(5)$ & $C(12)-C(13)$ & $1.379(8)$ \\
\hline $\mathrm{Ru}-\mathrm{Cl}(1)$ & $2.3698(12)$ & $C(13)-C(14)$ & $1.395(7)$ \\
\hline $\mathrm{Ru}-\mathrm{Cl}(2)$ & $2.3976(11)$ & $C(14)-C(15)$ & $1.375(7)$ \\
\hline $\mathrm{Ru}-\mathrm{P}(1)$ & $2.4386(14)$ & $C(16)-C(17)$ & $1.470(7)$ \\
\hline$P(1)-C(23)$ & $1.852(4)$ & $C(17)-C(22)$ & $1.398(6)$ \\
\hline $\mathrm{P}(1)-\mathrm{C}(35)$ & $1.850(5)$ & $\mathrm{C}(17)-\mathrm{C}(18)$ & $1.412(6)$ \\
\hline $\mathrm{P}(1)-\mathrm{C}(29)$ & $1.873(5)$ & $C(18)-C(19)$ & $1.385(7)$ \\
\hline $\mathrm{F}(1)-\mathrm{C}(5)$ & $1.339(6)$ & $C(19)-C(20)$ & $1.384(7)$ \\
\hline $\mathrm{F}(2)-\mathrm{C}(9)$ & $1.346(5)$ & $C(20)-C(21)$ & $1.388(7)$ \\
\hline $\mathrm{F}(3)-\mathrm{C}(11)$ & $1.349(6)$ & $C(21)-C(22)$ & $1.370(7)$ \\
\hline $\mathrm{F}(4)-\mathrm{C}(15)$ & $1.348(5)$ & $C(23)-C(28)$ & $1.525(6)$ \\
\hline $\mathrm{N}(1)-\mathrm{C}(1)$ & $1.343(6)$ & $C(23)-C(24)$ & $1.533(6)$ \\
\hline $\mathrm{N}(1)-\mathrm{C}(4)$ & $1.404(6)$ & $C(24)-C(25)$ & $1.526(6)$ \\
\hline $\mathrm{N}(1)-\mathrm{C}(2)$ & $1.475(7)$ & $C(25)-C(26)$ & $1.511(7)$ \\
\hline $\mathrm{N}(2)-\mathrm{C}(1)$ & $1.359(6)$ & $C(26)-C(27)$ & $1.517(7)$ \\
\hline $\mathrm{N}(2)-\mathrm{C}(10)$ & $1.399(6)$ & $C(27)-C(28)$ & $1.528(6)$ \\
\hline $\mathrm{N}(2)-\mathrm{C}(3)$ & $1.480(6)$ & $C(29)-C(30)$ & $1.529(6)$ \\
\hline $\mathrm{C}(2)-\mathrm{C}(3)$ & $1.498(7)$ & $C(29)-C(34)$ & $1.557(6)$ \\
\hline $\mathrm{C}(4)-\mathrm{C}(9)$ & $1.376(7)$ & $C(30)-C(31)$ & $1.541(6)$ \\
\hline $\mathrm{C}(4)-\mathrm{C}(5)$ & $1.407(7)$ & $C(31)-C(32)$ & $1.522(7)$ \\
\hline$C(5)-C(6)$ & $1.364(7)$ & $C(32)-C(33)$ & $1.514(7)$ \\
\hline$C(6)-C(7)$ & $1.374(8)$ & $\mathrm{C}(33)-\mathrm{C}(34)$ & $1.525(7)$ \\
\hline$C(7)-C(8)$ & $1.375(7)$ & $C(35)-C(36)$ & $1.523(7)$ \\
\hline $\mathrm{C}(8)-\mathrm{C}(9)$ & $1.377(7)$ & $\mathrm{C}(35)-\mathrm{C}(40)$ & $1.524(7)$ \\
\hline$C(10)-C(11)$ & $1.391(6)$ & $C(36)-C(37)$ & $1.530(7)$ \\
\hline$C(10)-C(15)$ & $1.394(7)$ & $\mathrm{C}(37)-\mathrm{C}(38)$ & $1.510(8)$ \\
\hline
\end{tabular}




\begin{tabular}{|c|c|c|c|}
\hline$C(38)-C(39)$ & $1.521(7)$ & $\mathrm{N}(2)-\mathrm{C}(3)-\mathrm{C}(2)$ & $102.7(4)$ \\
\hline \multirow[t]{2}{*}{$C(39)-C(40)$} & $1.516(7)$ & $\mathrm{C}(9)-\mathrm{C}(4)-\mathrm{N}(1)$ & $123.1(4)$ \\
\hline & & $C(9)-C(4)-C(5)$ & $115.8(5)$ \\
\hline $\mathrm{C}(16)-\mathrm{Ru}-\mathrm{C}(1)$ & $99.8(2)$ & $\mathrm{N}(1)-\mathrm{C}(4)-\mathrm{C}(5)$ & $120.7(5)$ \\
\hline $\mathrm{C}(16)-\mathrm{Ru}-\mathrm{Cl}(1)$ & $87.63(14)$ & $\mathrm{F}(1)-\mathrm{C}(5)-\mathrm{C}(6)$ & $120.1(5)$ \\
\hline $\mathrm{C}(1)-\mathrm{Ru}-\mathrm{Cl}(1)$ & $87.52(12)$ & $\mathrm{F}(1)-\mathrm{C}(5)-\mathrm{C}(4)$ & $117.2(5)$ \\
\hline $\mathrm{C}(16)-\mathrm{Ru}-\mathrm{Cl}(2)$ & $107.82(14)$ & $C(6)-C(5)-C(4)$ & $122.6(5)$ \\
\hline $\mathrm{C}(1)-\mathrm{Ru}-\mathrm{Cl}(2)$ & $83.44(12)$ & $C(5)-C(6)-C(7)$ & $118.6(5)$ \\
\hline $\mathrm{Cl}(1)-\mathrm{Ru}-\mathrm{Cl}(2)$ & $163.19(4)$ & $C(6)-C(7)-C(8)$ & $121.7(5)$ \\
\hline $\mathrm{C}(16)-\mathrm{Ru}-\mathrm{P}(1)$ & $93.83(15)$ & $C(9)-C(8)-C(7)$ & $118.0(5)$ \\
\hline $\mathrm{C}(1)-\mathrm{Ru}-\mathrm{P}(1)$ & $166.32(13)$ & $\mathrm{F}(2)-\mathrm{C}(9)-\mathrm{C}(4)$ & $118.3(5)$ \\
\hline $\mathrm{Cl}(1)-\mathrm{Ru}-\mathrm{P}(1)$ & $94.17(4)$ & $\mathrm{F}(2)-\mathrm{C}(9)-\mathrm{C}(8)$ & $118.3(5)$ \\
\hline $\mathrm{Cl}(2)-\mathrm{Ru}-\mathrm{P}(1)$ & $91.40(4)$ & $C(4)-C(9)-C(8)$ & $123.4(5)$ \\
\hline$C(23)-P(1)-C(35)$ & $106.1(2)$ & $C(11)-C(10)-C(15)$ & $115.7(5)$ \\
\hline$C(23)-P(1)-C(29)$ & $102.6(2)$ & $\mathrm{C}(11)-\mathrm{C}(10)-\mathrm{N}(2)$ & $121.2(4)$ \\
\hline$C(35)-P(1)-C(29)$ & $105.5(2)$ & $\mathrm{C}(15)-\mathrm{C}(10)-\mathrm{N}(2)$ & $123.0(4)$ \\
\hline $\mathrm{C}(23)-\mathrm{P}(1)-\mathrm{Ru}$ & $118.66(16)$ & $\mathrm{F}(3)-\mathrm{C}(11)-\mathrm{C}(12)$ & $120.0(4)$ \\
\hline$C(35)-P(1)-R u$ & $109.76(16)$ & $\mathrm{F}(3)-\mathrm{C}(11)-\mathrm{C}(10)$ & $116.5(5)$ \\
\hline$C(29)-P(1)-R u$ & $113.14(16)$ & $C(12)-C(11)-C(10)$ & $123.6(5)$ \\
\hline $\mathrm{C}(1)-\mathrm{N}(1)-\mathrm{C}(4)$ & $127.5(4)$ & $C(11)-C(12)-C(13)$ & $117.9(5)$ \\
\hline $\mathrm{C}(1)-\mathrm{N}(1)-\mathrm{C}(2)$ & $113.4(4)$ & $\mathrm{C}(12)-\mathrm{C}(13)-\mathrm{C}(14)$ & $122.3(5)$ \\
\hline $\mathrm{C}(4)-\mathrm{N}(1)-\mathrm{C}(2)$ & 119.1(4) & $C(15)-C(14)-C(13)$ & $116.8(5)$ \\
\hline $\mathrm{C}(1)-\mathrm{N}(2)-\mathrm{C}(10)$ & $126.4(4)$ & $\mathrm{F}(4)-\mathrm{C}(15)-\mathrm{C}(14)$ & $119.1(5)$ \\
\hline$C(1)-N(2)-C(3)$ & $112.3(4)$ & $\mathrm{F}(4)-\mathrm{C}(15)-\mathrm{C}(10)$ & $117.2(4)$ \\
\hline $\mathrm{C}(10)-\mathrm{N}(2)-\mathrm{C}(3)$ & $118.9(4)$ & $\mathrm{C}(14)-\mathrm{C}(15)-\mathrm{C}(10)$ & $123.7(5)$ \\
\hline $\mathrm{N}(1)-\mathrm{C}(1)-\mathrm{N}(2)$ & $106.8(4)$ & $C(17)-C(16)-R u$ & $135.0(4)$ \\
\hline $\mathrm{N}(1)-\mathrm{C}(1)-\mathrm{Ru}$ & $123.6(4)$ & $C(22)-C(17)-C(18)$ & $117.8(4)$ \\
\hline $\mathrm{N}(2)-\mathrm{C}(1)-\mathrm{Ru}$ & $129.5(3)$ & $C(22)-C(17)-C(16)$ & $124.0(4)$ \\
\hline $\mathrm{N}(1)-\mathrm{C}(2)-\mathrm{C}(3)$ & $102.4(4)$ & $C(18)-C(17)-C(16)$ & $118.2(4)$ \\
\hline
\end{tabular}




$\begin{array}{llll}\mathrm{C}(19)-\mathrm{C}(18)-\mathrm{C}(17) & 121.3(5) & \mathrm{C}(34)-\mathrm{C}(29)-\mathrm{P}(1) & 113.1(3) \\ \mathrm{C}(20)-\mathrm{C}(19)-\mathrm{C}(18) & 119.3(4) & \mathrm{C}(29)-\mathrm{C}(30)-\mathrm{C}(31) & 108.9(4) \\ \mathrm{C}(19)-\mathrm{C}(20)-\mathrm{C}(21) & 119.9(5) & \mathrm{C}(32)-\mathrm{C}(31)-\mathrm{C}(30) & 111.6(4) \\ \mathrm{C}(22)-\mathrm{C}(21)-\mathrm{C}(20) & 121.0(5) & \mathrm{C}(33)-\mathrm{C}(32)-\mathrm{C}(31) & 111.6(4) \\ \mathrm{C}(21)-\mathrm{C}(22)-\mathrm{C}(17) & 120.6(4) & \mathrm{C}(32)-\mathrm{C}(33)-\mathrm{C}(34) & 111.2(4) \\ \mathrm{C}(28)-\mathrm{C}(23)-\mathrm{C}(24) & 109.4(4) & \mathrm{C}(33)-\mathrm{C}(34)-\mathrm{C}(29) & 110.1(4) \\ \mathrm{C}(28)-\mathrm{C}(23)-\mathrm{P}(1) & 110.4(3) & \mathrm{C}(36)-\mathrm{C}(35)-\mathrm{C}(40) & 112.0(4) \\ \mathrm{C}(24)-\mathrm{C}(23)-\mathrm{P}(1) & 117.1(3) & \mathrm{C}(36)-\mathrm{C}(35)-\mathrm{P}(1) & 111.9(4) \\ \mathrm{C}(25)-\mathrm{C}(24)-\mathrm{C}(23) & 111.3(4) & \mathrm{C}(40)-\mathrm{C}(35)-\mathrm{P}(1) & 114.7(3) \\ \mathrm{C}(26)-\mathrm{C}(25)-\mathrm{C}(24) & 112.3(4) & \mathrm{C}(35)-\mathrm{C}(36)-\mathrm{C}(37) & 112.2(4) \\ \mathrm{C}(25)-\mathrm{C}(26)-\mathrm{C}(27) & 110.9(4) & \mathrm{C}(38)-\mathrm{C}(37)-\mathrm{C}(36) & 111.3(4) \\ \mathrm{C}(26)-\mathrm{C}(27)-\mathrm{C}(28) & 110.1(4) & \mathrm{C}(37)-\mathrm{C}(38)-\mathrm{C}(39) & 111.2(4) \\ \mathrm{C}(23)-\mathrm{C}(28)-\mathrm{C}(27) & 111.2(4) & \mathrm{C}(40)-\mathrm{C}(39)-\mathrm{C}(38) & 111.1(4) \\ \mathrm{C}(30)-\mathrm{C}(29)-\mathrm{C}(34) & 107.9(4) & \mathrm{C}(39)-\mathrm{C}(40)-\mathrm{C}(35) & 111.6(4) \\ \mathrm{C}(30)-\mathrm{C}(29)-\mathrm{P}(1) & 119.3(3) & & \end{array}$


Table 5. Anisotropic displacement parameters $\left(\AA^{2} \mathbf{x} 10^{4}\right)$ for CCDC 279454. The anisotropic displacement factor exponent takes the form: $-2 \pi^{2}\left[h^{2} a^{* 2} U 11+\ldots+2 h k\right.$ a* $b^{*}$ $\left.\mathbf{U}^{12}\right]$

\begin{tabular}{|c|c|c|c|c|c|c|}
\hline & $\mathrm{U}^{11}$ & $\mathrm{U}^{22}$ & $\mathrm{U}^{33}$ & $\mathrm{U}^{23}$ & $\mathrm{U}^{13}$ & $\mathrm{U}^{12}$ \\
\hline $\mathrm{Ru}$ & $122(2)$ & $112(2)$ & $159(2)$ & $8(1)$ & $-16(2)$ & $7(2)$ \\
\hline $\mathrm{Cl}(1)$ & $248(7)$ & $152(6)$ & $228(6)$ & $-7(5)$ & $-31(5)$ & $48(5)$ \\
\hline $\mathrm{Cl}(2)$ & $165(6)$ & $212(6)$ & $196(6)$ & $-22(5)$ & $-1(5)$ & $58(5)$ \\
\hline $\mathrm{P}(1)$ & $151(7)$ & $121(6)$ & $170(6)$ & $-13(5)$ & $-17(5)$ & $14(5)$ \\
\hline $\mathrm{F}(1)$ & $251(17)$ & $223(16)$ & $423(19)$ & $20(14)$ & $-42(14)$ & $66(13)$ \\
\hline $\mathrm{F}(2)$ & $329(18)$ & $192(16)$ & 424(19) & $6(14)$ & $-15(14)$ & $90(14)$ \\
\hline $\mathrm{F}(3)$ & $267(17)$ & $244(17)$ & $435(19)$ & $-57(14)$ & $-68(14)$ & $130(14)$ \\
\hline $\mathrm{F}(4)$ & $247(17)$ & $182(16)$ & $405(18)$ & $-17(13)$ & $-41(14)$ & $88(13)$ \\
\hline $\mathrm{N}(1)$ & $200(20)$ & $180(20)$ & $190(20)$ & $-18(17)$ & $-12(17)$ & $-46(18)$ \\
\hline $\mathrm{N}(2)$ & $190(20)$ & $160(20)$ & $160(20)$ & $-24(17)$ & $-48(17)$ & $42(18)$ \\
\hline $\mathrm{C}(1)$ & $150(30)$ & $90(20)$ & $210(30)$ & $-16(19)$ & $20(20)$ & $35(19)$ \\
\hline $\mathrm{C}(2)$ & $380(30)$ & $320(30)$ & $190(30)$ & $20(20)$ & $-50(20)$ & $-60(30)$ \\
\hline$C(3)$ & $320(30)$ & $270(30)$ & $190(30)$ & $20(20)$ & $-70(20)$ & $60(20)$ \\
\hline$C(4)$ & $200(30)$ & $190(30)$ & $140(20)$ & $20(20)$ & $20(20)$ & $-40(20)$ \\
\hline$C(5)$ & $230(30)$ & $240(30)$ & $230(30)$ & $30(20)$ & $30(20)$ & $20(20)$ \\
\hline$C(6)$ & $410(40)$ & $180(30)$ & $250(30)$ & $20(20)$ & $-40(20)$ & $-10(30)$ \\
\hline$C(7)$ & $310(30)$ & $240(30)$ & $310(30)$ & $60(20)$ & $-80(20)$ & $-130(30)$ \\
\hline$C(8)$ & $180(30)$ & $350(30)$ & $260(30)$ & $110(20)$ & $-60(20)$ & $-50(20)$ \\
\hline $\mathrm{C}(9)$ & $240(30)$ & $220(30)$ & $200(30)$ & $40(20)$ & $0(20)$ & $30(20)$ \\
\hline$C(10)$ & $170(30)$ & $160(30)$ & $210(30)$ & $-40(20)$ & $-70(20)$ & $20(20)$ \\
\hline$C(11)$ & $180(30)$ & $280(30)$ & $240(30)$ & $-70(20)$ & $-70(20)$ & $0(20)$ \\
\hline$C(12)$ & $140(30)$ & $440(40)$ & $260(30)$ & $-110(30)$ & $-70(20)$ & $10(20)$ \\
\hline$C(13)$ & $200(30)$ & $490(40)$ & $320(30)$ & $-20(30)$ & $-70(20)$ & $-170(30)$ \\
\hline$C(14)$ & $340(30)$ & $240(30)$ & $280(30)$ & $-20(20)$ & $-70(20)$ & $-110(30)$ \\
\hline
\end{tabular}




\begin{tabular}{|c|c|c|c|c|c|c|}
\hline$C(15)$ & $150(30)$ & $330(30)$ & $220(30)$ & $-30(20)$ & $-70(20)$ & $20(20)$ \\
\hline$C(16)$ & $310(30)$ & $120(20)$ & $200(30)$ & $-64(19)$ & $-160(20)$ & $170(20)$ \\
\hline$C(17)$ & $170(30)$ & $200(30)$ & $160(20)$ & $-10(20)$ & $-55(19)$ & $-10(20)$ \\
\hline$C(18)$ & 190(30) & $190(30)$ & $320(30)$ & $-50(20)$ & $-40(20)$ & $30(20)$ \\
\hline$C(19)$ & $220(30)$ & $230(30)$ & $330(30)$ & $40(20)$ & $-50(20)$ & $-100(20)$ \\
\hline$C(20)$ & $210(30)$ & $180(30)$ & $330(30)$ & $20(20)$ & $-80(20)$ & $-30(20)$ \\
\hline $\mathrm{C}(21)$ & $230(30)$ & $160(30)$ & $320(30)$ & $-40(20)$ & $-70(20)$ & $-50(20)$ \\
\hline$C(22)$ & $180(30)$ & $140(30)$ & $250(30)$ & $-20(20)$ & $-60(20)$ & $0(20)$ \\
\hline$C(23)$ & $150(20)$ & $130(20)$ & $140(20)$ & $-21(18)$ & $-11(19)$ & $50(19)$ \\
\hline$C(24)$ & $140(30)$ & $210(30)$ & $240(30)$ & $-20(20)$ & $30(20)$ & $-10(20)$ \\
\hline$C(25)$ & $240(30)$ & $160(30)$ & $300(30)$ & $-60(20)$ & $20(20)$ & $20(20)$ \\
\hline$C(26)$ & $230(30)$ & $160(30)$ & $300(30)$ & $-50(20)$ & $0(20)$ & $60(20)$ \\
\hline$C(27)$ & $140(20)$ & $200(30)$ & $200(30)$ & $-20(20)$ & 11(19) & $10(20)$ \\
\hline$C(28)$ & $210(30)$ & $150(30)$ & $170(20)$ & $34(19)$ & $-40(20)$ & $10(20)$ \\
\hline$C(29)$ & $140(20)$ & $80(20)$ & $250(30)$ & $-50(19)$ & $-50(20)$ & $69(19)$ \\
\hline $\mathrm{C}(30)$ & $150(30)$ & $100(20)$ & $220(30)$ & $-2(19)$ & $-40(20)$ & $-16(19)$ \\
\hline $\mathrm{C}(31)$ & $350(30)$ & $150(30)$ & $270(30)$ & $40(20)$ & $-110(20)$ & $-30(20)$ \\
\hline$C(32)$ & $300(30)$ & $120(30)$ & $400(30)$ & $20(20)$ & $-160(20)$ & $40(20)$ \\
\hline $\mathrm{C}(33)$ & $330(30)$ & $180(30)$ & $350(30)$ & $-60(20)$ & $-110(20)$ & $110(20)$ \\
\hline$C(34)$ & $310(30)$ & $150(30)$ & $230(30)$ & $-30(20)$ & $-40(20)$ & $120(20)$ \\
\hline$C(35)$ & $110(20)$ & $220(30)$ & 190(30) & $-40(20)$ & $26(19)$ & $-30(20)$ \\
\hline$C(36)$ & $210(30)$ & $180(30)$ & $210(30)$ & $20(20)$ & $-30(20)$ & $0(20)$ \\
\hline $\mathrm{C}(37)$ & $270(30)$ & $230(30)$ & $250(30)$ & $-30(20)$ & $40(20)$ & $-70(20)$ \\
\hline$C(38)$ & $210(30)$ & $150(30)$ & $370(30)$ & $-40(20)$ & $-50(20)$ & $-10(20)$ \\
\hline $\mathrm{C}(39)$ & $180(30)$ & $190(30)$ & $320(30)$ & $-70(20)$ & $-100(20)$ & $10(20)$ \\
\hline $\mathrm{C}(40)$ & $200(30)$ & $190(30)$ & $250(30)$ & $-10(20)$ & $-60(20)$ & $-10(20)$ \\
\hline
\end{tabular}


Table 6. Selected torsion angles $\left[{ }^{\circ}\right]$ for CCDC 279454.

$\mathrm{N}(2)-\mathrm{C}(1)-\mathrm{Ru}-\mathrm{C}(16)$

$-19.5(4)$

$\mathrm{C}(1)-\mathrm{N}(1)-\mathrm{C}(4)-\mathrm{C}(9)$

$92.5(6)$

$\mathrm{C}(1)-\mathrm{N}(2)-\mathrm{C}(10)-\mathrm{C}(15)$

$-97.4(6)$ 
Difluoro-ether derived ruthenium complex 6

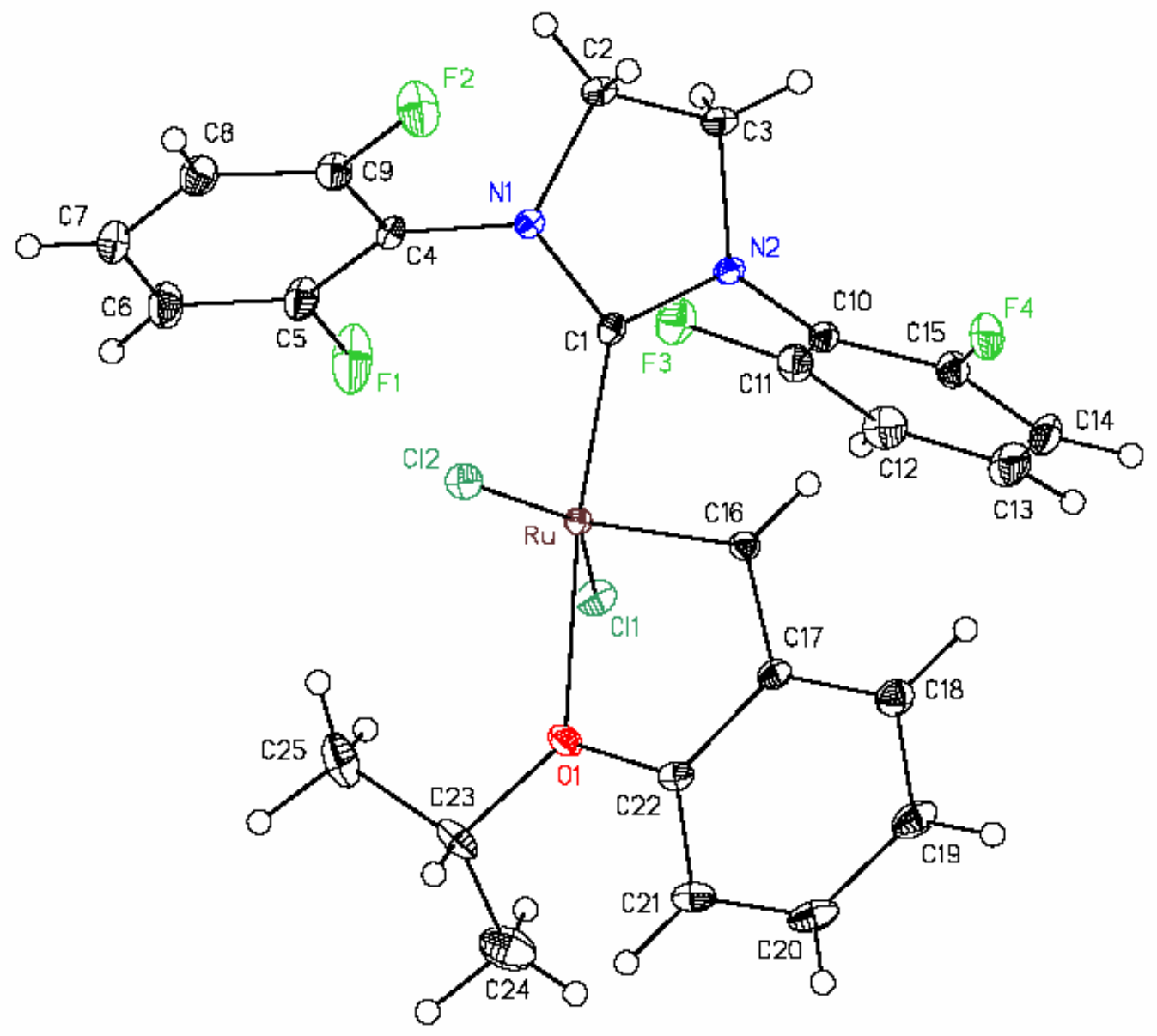


Table 1. Crystal data and structure refinement for CCDC 277100.

Empirical formula

Formula weight

Crystallization Solvent

Crystal Habit

Crystal size

Crystal color
$\mathrm{C}_{25} \mathrm{H}_{22} \mathrm{~F}_{4} \mathrm{~N}_{2} \mathrm{OCl}_{2} \mathrm{Ru}$

614.42

Dichloromethane/pentane

Prism

$0.39 \times 0.28 \times 0.27 \mathrm{~mm}^{3}$

Green-brown

\section{Data Collection}

Type of diffractometer

Wavelength

Data Collection Temperature

$\theta$ range for 22539 reflections used

in lattice determination

Unit cell dimensions

Volume

Z

Crystal system

Space group

Density (calculated)

$\mathrm{F}(000)$

Data collection program

$\theta$ range for data collection
Bruker SMART 1000

$0.71073 \AA \mathrm{MoK} \alpha$

100(2) K

2.57 to $47.36^{\circ}$

$a=15.8715(4) \AA$

$\mathrm{b}=13.4827(4) \AA$

$\beta=90.1460(10)^{\circ}$
2401.22(11) $\AA^{3}$

4

Monoclinic

$\mathrm{P} 2{ }_{1} / \mathrm{c}$

$1.700 \mathrm{Mg} / \mathrm{m}^{3}$

1232

Bruker SMART v5.630

1.98 to $47.41^{\circ}$ 
Completeness to $\theta=47.41^{\circ}$

Index ranges

Data collection scan type

Data reduction program

Reflections collected

Independent reflections

Absorption coefficient

Absorption correction

Max. and min. transmission
$87.8 \%$

$-30 \leq \mathrm{h} \leq 32,-26 \leq \mathrm{k} \leq 27,-22 \leq 1 \leq 19$

$\omega$ scans at $5 \phi$ settings

Bruker SAINT v6.45A

51113

$19604\left[\mathrm{R}_{\mathrm{int}}=0.0609\right]$

$0.929 \mathrm{~mm}^{-1}$

None

0.7876 and 0.7134 


\section{Table 1 (cont.)}

\section{Structure solution and Refinement}

\begin{tabular}{ll} 
Structure solution program & Bruker XS v6.12 \\
Primary solution method & Direct methods \\
Secondary solution method & Difference Fourier map \\
Hydrogen placement & Difference Fourier map \\
Structure refinement program & Bruker XL v6.12 \\
Refinement method & Full matrix least-squares on F ${ }^{2}$ \\
Data / restraints / parameters & $19604 / 0 / 404$ \\
Treatment of hydrogen atoms & Unrestrained \\
Goodness-of-fit on F & 1.174 \\
Final R indices [I $>2 \sigma(\mathrm{I}), 13727$ reflections $]$ & $\mathrm{R} 1=0.0353, w \mathrm{R} 2=0.0677$ \\
R indices (all data) & $\mathrm{R} 1=0.0602, w \mathrm{R} 2=0.0717$ \\
Type of weighting scheme used & Sigma \\
Weighting scheme used & $w=1 / \sigma^{2}\left(\mathrm{Fo}^{2}\right)$ \\
Max shift/error & 0.002 \\
\hline argerage shift/error & 0.000 \\
\hline
\end{tabular}

\section{Special Refinement Details}

Interesting features of this structure include the torsion angle around the $\mathrm{Ru}-\mathrm{C}(1)$ bond and the twist of the halogenated mesityl ring planes with respect to the dihydroimidazole plane (see Table 7 for torsion and dihedral angles). With respect to the first; in other similar $\mathrm{H}_{2}$ Imes compounds the torion angle tends to be near zero degrees, here it is near $40^{\circ}$ as shown in the fourth figure. Regarding the second; the mesityl rings tend towards $90^{\circ}$ where one twists significantly at $64.4^{\circ}$ here (see fifth figure) and the other one is $88.7^{\circ}$ (last figure). These features combine to place $\mathrm{F}(1)$ at $3.2 \AA$ from $\mathrm{Ru}$.

Refinement of $\mathrm{F}^{2}$ against ALL reflections. The weighted R-factor $(w \mathrm{R})$ and goodness of fit (S) are based on $\mathrm{F}^{2}$, conventional R-factors $(\mathrm{R})$ are based on $\mathrm{F}$, with $\mathrm{F}$ set to zero for negative $\mathrm{F}^{2}$. The threshold expression of $\mathrm{F}^{2}$ 
$>2 \sigma\left(\mathrm{F}^{2}\right)$ is used only for calculating R-factors(gt) etc. and is not relevant to the choice of reflections for refinement. R-factors based on $\mathrm{F}^{2}$ are statistically about twice as large as those based on $\mathrm{F}$, and R-factors based on ALL data will be even larger.

All esds (except the esd in the dihedral angle between two 1.s. planes) are estimated using the full covariance matrix. The cell esds are taken into account individually in the estimation of esds in distances, angles and torsion angles; correlations between esds in cell parameters are only used when they are defined by crystal symmetry. An approximate (isotropic) treatment of cell esds is used for estimating esds involving 1.s. planes. 


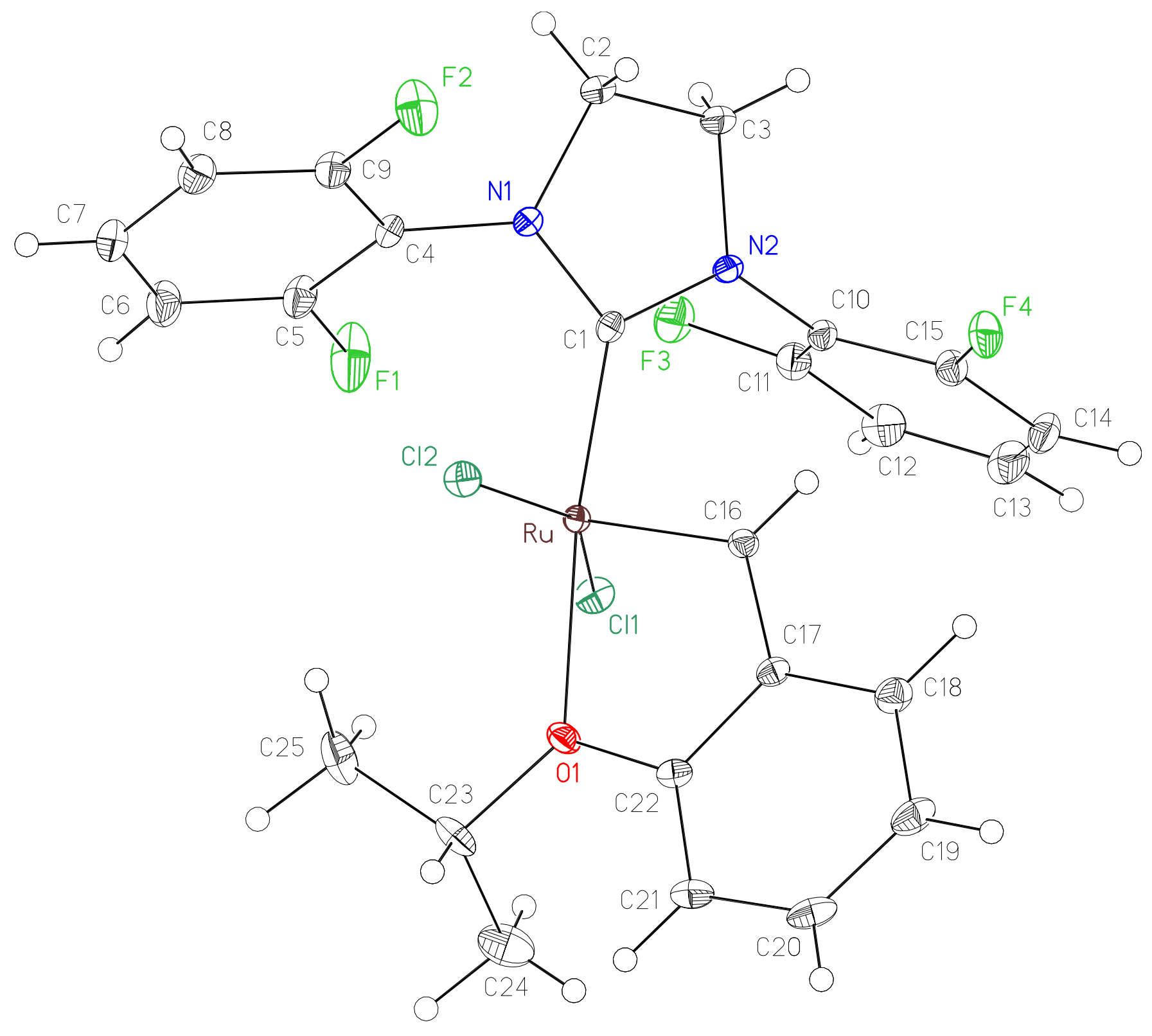




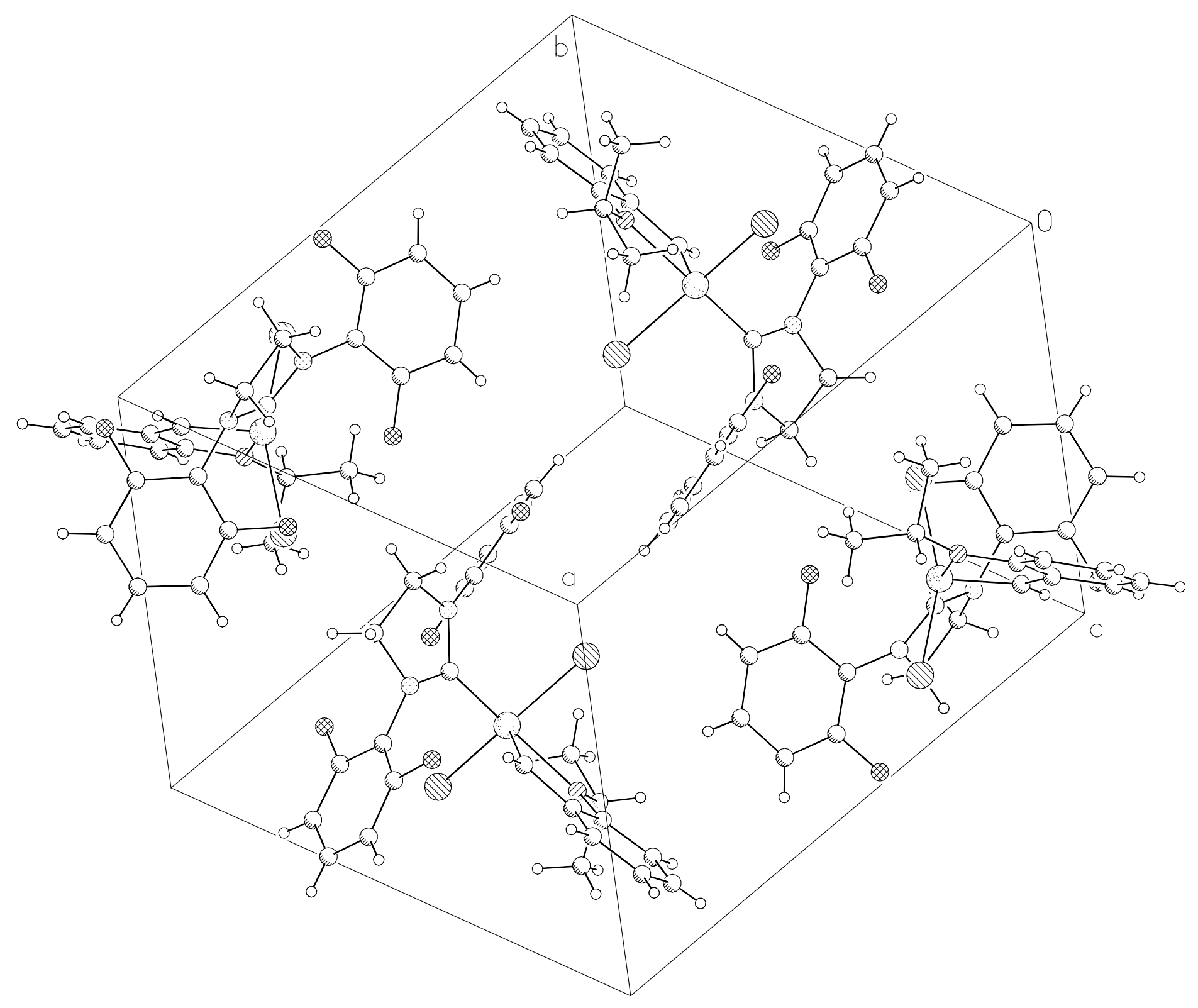



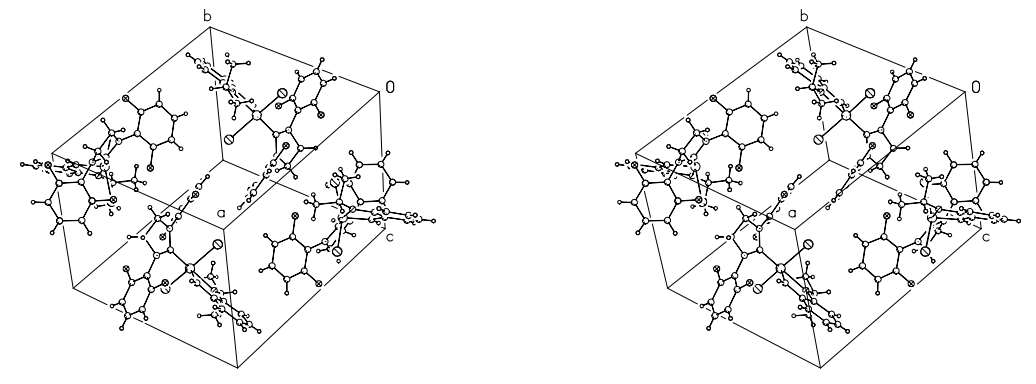


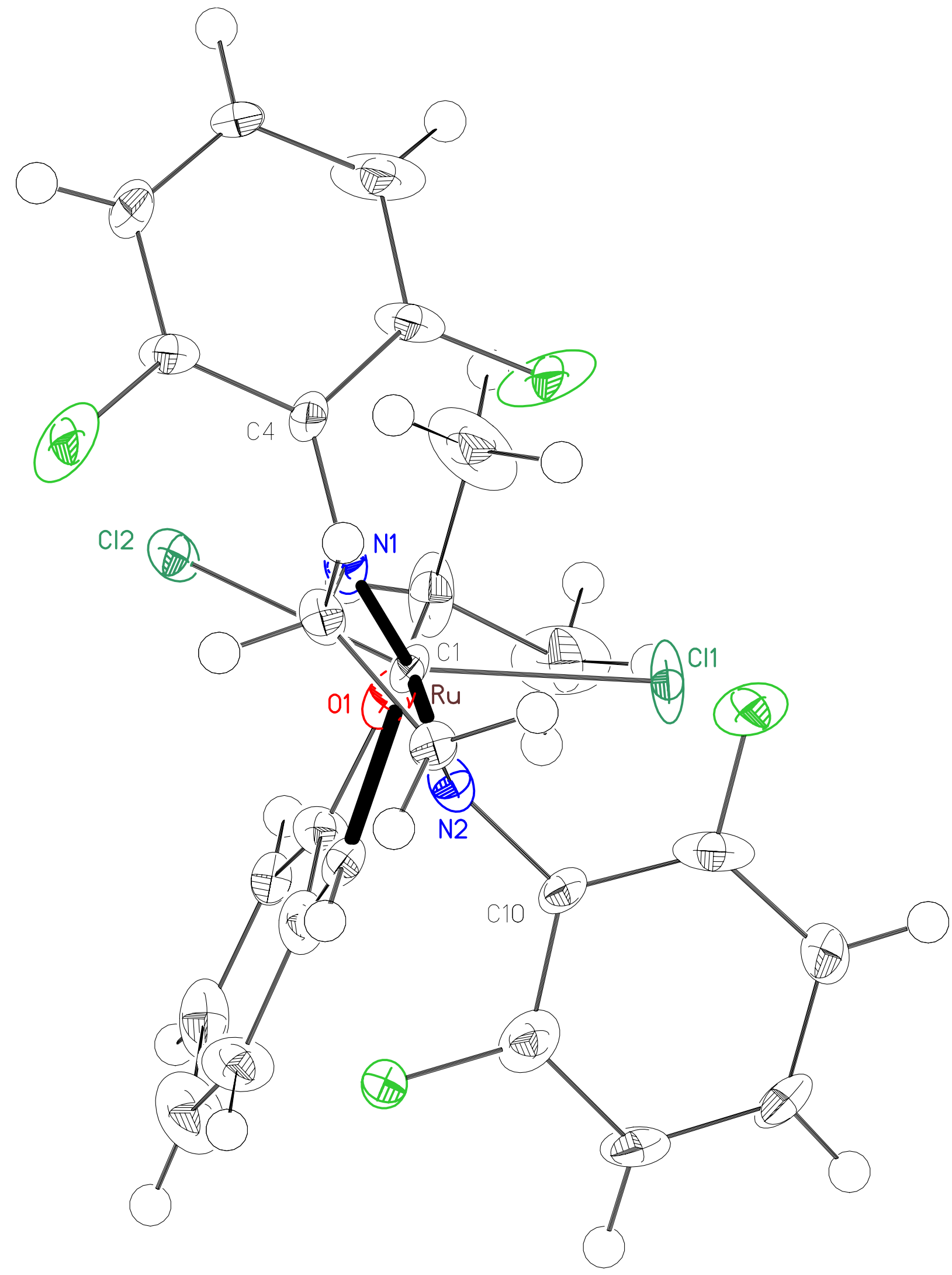




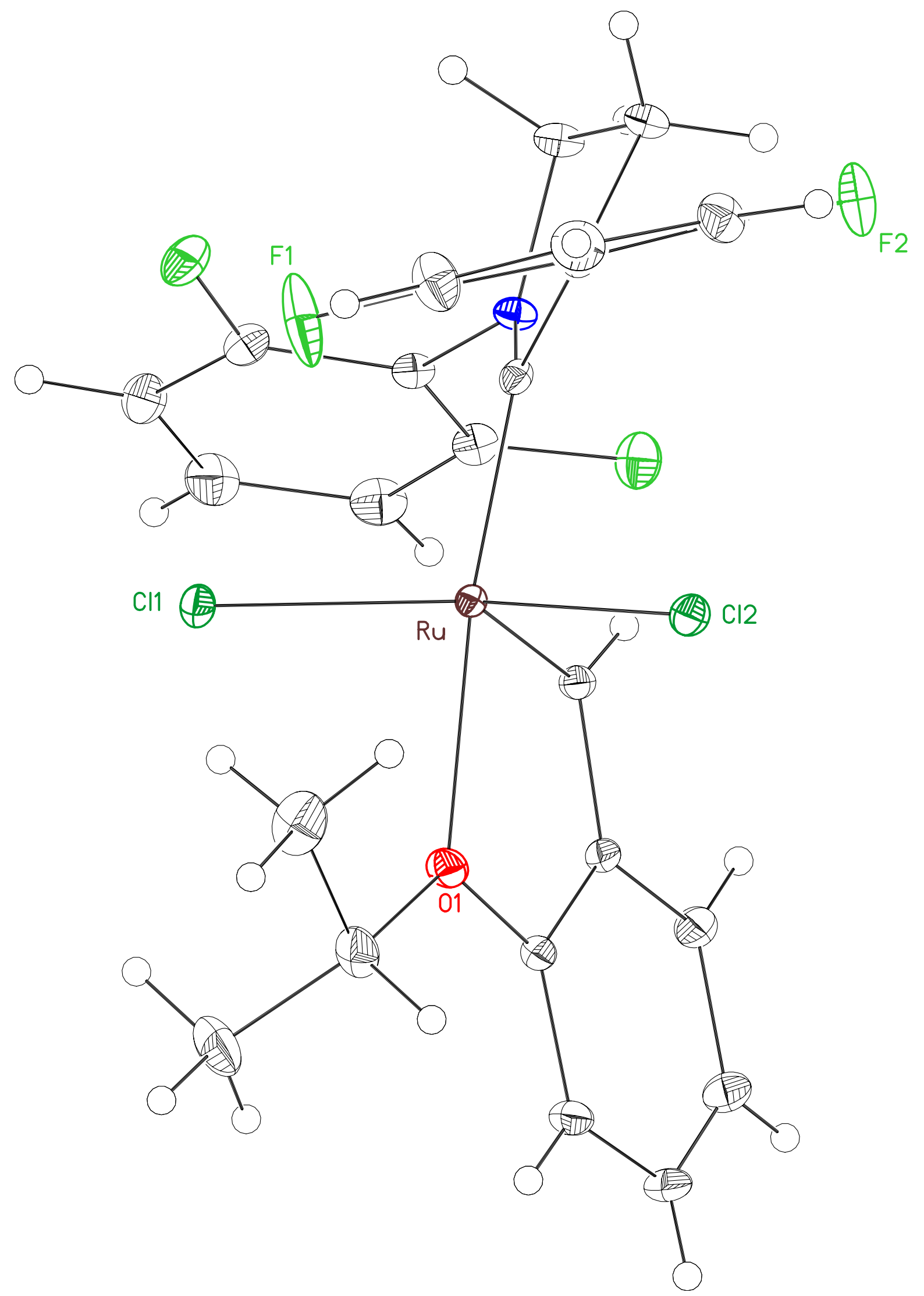

S36 


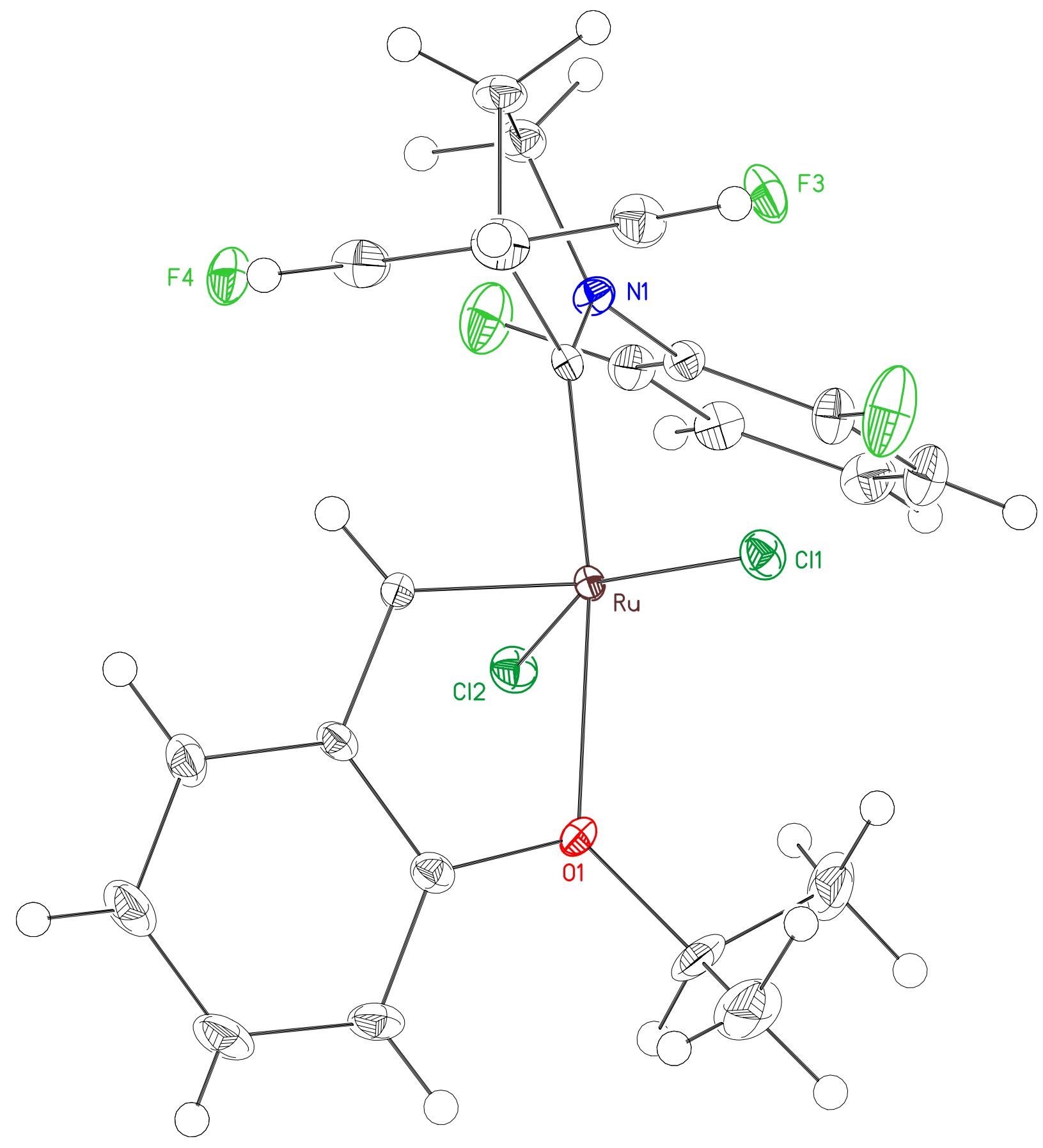


Table 2. Atomic coordinates $\left(\mathrm{x} \mathrm{10}^{4}\right)$ and equivalent isotropic displacement parameters $\left(\AA^{2} \times 1^{3}\right)$ for CCDC 277100 . $U(e q)$ is defined as the trace of the orthogonalized $U^{i j}$ tensor.

\begin{tabular}{|c|c|c|c|c|}
\hline & $\mathrm{x}$ & $\mathrm{y}$ & $\mathrm{z}$ & $\mathrm{U}_{\text {eq }}$ \\
\hline $\mathrm{Ru}$ & 7341(1) & $9866(1)$ & $3417(1)$ & $7(1)$ \\
\hline $\mathrm{Cl}(1)$ & $8038(1)$ & $8890(1)$ & 4821(1) & $13(1)$ \\
\hline $\mathrm{Cl}(2)$ & $6146(1)$ & $10514(1)$ & $2466(1)$ & $12(1)$ \\
\hline $\mathrm{F}(1)$ & $6700(1)$ & $7622(1)$ & 2964(1) & $30(1)$ \\
\hline $\mathrm{F}(2)$ & $6068(1)$ & $9366(1)$ & $-544(1)$ & $22(1)$ \\
\hline $\mathrm{F}(3)$ & $9026(1)$ & $7607(1)$ & $3002(1)$ & $18(1)$ \\
\hline $\mathrm{F}(4)$ & $9687(1)$ & $10769(1)$ & 1519(1) & $17(1)$ \\
\hline $\mathrm{O}(1)$ & $6948(1)$ & 10831(1) & $4958(1)$ & $12(1)$ \\
\hline $\mathrm{N}(1)$ & $7275(1)$ & $8786(1)$ & $1096(1)$ & $10(1)$ \\
\hline $\mathrm{N}(2)$ & $8570(1)$ & $9179(1)$ & $1563(1)$ & $10(1)$ \\
\hline$C(1)$ & $7757(1)$ & 9196(1) & 1981(1) & $9(1)$ \\
\hline$C(2)$ & $7749(1)$ & $8626(1)$ & $-24(1)$ & $12(1)$ \\
\hline$C(3)$ & $8652(1)$ & $8620(1)$ & $432(1)$ & $13(1)$ \\
\hline$C(4)$ & $6423(1)$ & $8501(1)$ & $1200(1)$ & $10(1)$ \\
\hline$C(5)$ & $6136(1)$ & 7904(1) & $2123(1)$ & $15(1)$ \\
\hline$C(6)$ & $5314(1)$ & $7588(1)$ & $2222(1)$ & $19(1)$ \\
\hline$C(7)$ & $4737(1)$ & $7872(1)$ & $1348(1)$ & $17(1)$ \\
\hline$C(8)$ & $4987(1)$ & $8474(1)$ & $411(1)$ & $16(1)$ \\
\hline$C(9)$ & $5820(1)$ & $8768(1)$ & $353(1)$ & $13(1)$ \\
\hline$C(10)$ & 9294(1) & $9220(1)$ & $2314(1)$ & $10(1)$ \\
\hline$C(11)$ & $9514(1)$ & $8425(1)$ & $3044(1)$ & $13(1)$ \\
\hline$C(12)$ & $10217(1)$ & $8442(1)$ & $3782(1)$ & $17(1)$ \\
\hline$C(13)$ & $10726(1)$ & $9284(1)$ & $3769(1)$ & $19(1)$ \\
\hline$C(14)$ & $10554(1)$ & $10076(1)$ & $3015(1)$ & $17(1)$ \\
\hline$C(15)$ & $9853(1)$ & $10017(1)$ & $2289(1)$ & $13(1)$ \\
\hline
\end{tabular}




$\begin{array}{lllll}\mathrm{C}(16) & 8026(1) & 10941(1) & 3182(1) & 9(1) \\ \mathrm{C}(17) & 7916(1) & 11801(1) & 3941(1) & 10(1) \\ \mathrm{C}(18) & 8358(1) & 12690(1) & 3758(1) & 14(1) \\ \mathrm{C}(19) & 8185(1) & 13517(1) & 4456(1) & 17(1) \\ \mathrm{C}(20) & 7563(1) & 13461(1) & 5321(1) & 17(1) \\ \mathrm{C}(21) & 7127(1) & 12580(1) & 5548(1) & 14(1) \\ \mathrm{C}(22) & 7315(1) & 11749(1) & 4859(1) & 10(1) \\ \mathrm{C}(23) & 6362(1) & 10610(1) & 5940(1) & 16(1) \\ \mathrm{C}(24) & 6847(1) & 10567(1) & 7111(1) & 26(1) \\ \mathrm{C}(25) & 5950(1) & 9630(1) & 5640(1) & 22(1) \\ \end{array}$


Table 3. Selected bond lengths $[\AA]]$ and angles $\left[^{\circ}\right]$ for CCDC 277100.

\begin{tabular}{lccc}
\hline $\mathrm{Ru}-\mathrm{C}(16)$ & $1.8311(11)$ & $\mathrm{C}(16)-\mathrm{Ru}-\mathrm{C}(1)$ & $92.60(5)$ \\
$\mathrm{Ru}-\mathrm{C}(1)$ & $1.9628(11)$ & $\mathrm{C}(16)-\mathrm{Ru}-\mathrm{O}(1)$ & $79.56(4)$ \\
$\mathrm{Ru}-\mathrm{O}(1)$ & $2.2528(8)$ & $\mathrm{C}(1)-\mathrm{Ru}-\mathrm{O}(1)$ & $171.85(4)$ \\
$\mathrm{Ru}-\mathrm{Cl}(1)$ & $2.3287(3)$ & $\mathrm{C}(16)-\mathrm{Ru}-\mathrm{Cl}(1)$ & $105.30(3)$ \\
$\mathrm{Ru}-\mathrm{Cl}(2)$ & $2.3443(3)$ & $\mathrm{C}(1)-\mathrm{Ru}-\mathrm{Cl}(1)$ & $97.81(3)$ \\
& & $\mathrm{O}(1)-\mathrm{Ru}-\mathrm{Cl}(1)$ & $86.52(2)$ \\
& & $\mathrm{C}(16)-\mathrm{Ru}-\mathrm{Cl}(2)$ & $96.83(3)$ \\
& & $\mathrm{C}(1)-\mathrm{Ru}-\mathrm{Cl}(2)$ & $94.04(3)$ \\
& & $\mathrm{O}(1)-\mathrm{Ru}-\mathrm{Cl}(2)$ & $84.76(2)$ \\
& & $\mathrm{Cl}(1)-\mathrm{Ru}-\mathrm{Cl}(2)$ & $154.289(11)$
\end{tabular}

Non-bonded distance

Ru-F(1) 3.2311(10) 
Table 4. Bond lengths $[\AA ̊]$ and angles $\left[^{\circ}\right]$ for TAR05 (CCDC 277100).

\begin{tabular}{|c|c|c|c|}
\hline $\mathrm{Ru}-\mathrm{C}(16)$ & $1.8311(11)$ & $C(7)-C(8)$ & $1.3878(19)$ \\
\hline $\mathrm{Ru}-\mathrm{C}(1)$ & $1.9628(11)$ & $\mathrm{C}(7)-\mathrm{H}(7)$ & $0.867(18)$ \\
\hline $\mathrm{Ru}-\mathrm{O}(1)$ & $2.2528(8)$ & $\mathrm{C}(8)-\mathrm{C}(9)$ & $1.3814(17)$ \\
\hline $\mathrm{Ru}-\mathrm{Cl}(1)$ & $2.3287(3)$ & $\mathrm{C}(8)-\mathrm{H}(8)$ & $0.893(17)$ \\
\hline $\mathrm{Ru}-\mathrm{Cl}(2)$ & $2.3443(3)$ & $\mathrm{C}(10)-\mathrm{C}(11)$ & $1.3939(16)$ \\
\hline$F(1)-C(5)$ & $1.3538(13)$ & $C(10)-C(15)$ & $1.3938(16)$ \\
\hline $\mathrm{F}(2)-\mathrm{C}(9)$ & $1.3490(13)$ & $\mathrm{C}(11)-\mathrm{C}(12)$ & $1.3870(17)$ \\
\hline $\mathrm{F}(3)-\mathrm{C}(11)$ & $1.3485(14)$ & $\mathrm{C}(12)-\mathrm{C}(13)$ & $1.393(2)$ \\
\hline $\mathrm{F}(4)-\mathrm{C}(15)$ & $1.3566(13)$ & $\mathrm{C}(12)-\mathrm{H}(12)$ & $0.964(18)$ \\
\hline $\mathrm{O}(1)-\mathrm{C}(22)$ & $1.3728(13)$ & $\mathrm{C}(13)-\mathrm{C}(14)$ & $1.3896(19)$ \\
\hline $\mathrm{O}(1)-\mathrm{C}(23)$ & $1.4742(14)$ & $\mathrm{C}(13)-\mathrm{H}(13)$ & $0.881(17)$ \\
\hline $\mathrm{N}(1)-\mathrm{C}(1)$ & $1.3677(13)$ & $\mathrm{C}(14)-\mathrm{C}(15)$ & $1.3802(16)$ \\
\hline $\mathrm{N}(1)-\mathrm{C}(4)$ & $1.4115(14)$ & $\mathrm{C}(14)-\mathrm{H}(14)$ & $0.949(17)$ \\
\hline $\mathrm{N}(1)-\mathrm{C}(2)$ & $1.4816(15)$ & $\mathrm{C}(16)-\mathrm{C}(17)$ & $1.4491(14)$ \\
\hline $\mathrm{N}(2)-\mathrm{C}(1)$ & $1.3744(14)$ & $\mathrm{C}(16)-\mathrm{H}(16)$ & $0.878(16)$ \\
\hline $\mathrm{N}(2)-\mathrm{C}(10)$ & $1.4245(13)$ & $\mathrm{C}(17)-\mathrm{C}(18)$ & $1.4038(16)$ \\
\hline $\mathrm{N}(2)-\mathrm{C}(3)$ & $1.4821(14)$ & $\mathrm{C}(17)-\mathrm{C}(22)$ & $1.4073(15)$ \\
\hline$C(2)-C(3)$ & $1.5209(17)$ & $\mathrm{C}(18)-\mathrm{C}(19)$ & $1.3901(17)$ \\
\hline $\mathrm{C}(2)-\mathrm{H}(2 \mathrm{~A})$ & $0.979(17)$ & $\mathrm{C}(18)-\mathrm{H}(18)$ & $0.982(16)$ \\
\hline $\mathrm{C}(2)-\mathrm{H}(2 \mathrm{~B})$ & $0.917(17)$ & $C(19)-C(20)$ & $1.389(2)$ \\
\hline $\mathrm{C}(3)-\mathrm{H}(3 \mathrm{~A})$ & $1.054(16)$ & $\mathrm{C}(19)-\mathrm{H}(19)$ & $0.903(16)$ \\
\hline $\mathrm{C}(3)-\mathrm{H}(3 \mathrm{~B})$ & $0.943(16)$ & $C(20)-C(21)$ & $1.3986(18)$ \\
\hline$C(4)-C(5)$ & $1.3890(16)$ & $\mathrm{C}(20)-\mathrm{H}(20)$ & $0.773(17)$ \\
\hline $\mathrm{C}(4)-\mathrm{C}(9)$ & $1.3945(15)$ & $\mathrm{C}(21)-\mathrm{C}(22)$ & $1.3936(15)$ \\
\hline$C(5)-C(6)$ & $1.3770(18)$ & $\mathrm{C}(21)-\mathrm{H}(21)$ & $0.934(18)$ \\
\hline$C(6)-C(7)$ & $1.3929(18)$ & $\mathrm{C}(23)-\mathrm{C}(25)$ & $1.512(2)$ \\
\hline $\mathrm{C}(6)-\mathrm{H}(6)$ & $0.89(2)$ & $C(23)-C(24)$ & $1.5228(19)$ \\
\hline
\end{tabular}




\begin{tabular}{|c|c|c|c|}
\hline $\mathrm{C}(23)-\mathrm{H}(23)$ & $0.929(16)$ & $\mathrm{N}(2)-\mathrm{C}(1)-\mathrm{Ru}$ & $127.25(7)$ \\
\hline $\mathrm{C}(24)-\mathrm{H}(24 \mathrm{~A})$ & $0.986(19)$ & $\mathrm{N}(1)-\mathrm{C}(2)-\mathrm{C}(3)$ & $101.25(8)$ \\
\hline $\mathrm{C}(24)-\mathrm{H}(24 \mathrm{~B})$ & $1.02(2)$ & $\mathrm{N}(1)-\mathrm{C}(2)-\mathrm{H}(2 \mathrm{~A})$ & $108.5(10)$ \\
\hline $\mathrm{C}(24)-\mathrm{H}(24 \mathrm{C})$ & $0.86(2)$ & $\mathrm{C}(3)-\mathrm{C}(2)-\mathrm{H}(2 \mathrm{~A})$ & $111.0(10)$ \\
\hline $\mathrm{C}(25)-\mathrm{H}(25 \mathrm{~A})$ & $0.980(16)$ & $\mathrm{N}(1)-\mathrm{C}(2)-\mathrm{H}(2 \mathrm{~B})$ & $110.6(11)$ \\
\hline $\mathrm{C}(25)-\mathrm{H}(25 \mathrm{~B})$ & $1.02(2)$ & $\mathrm{C}(3)-\mathrm{C}(2)-\mathrm{H}(2 \mathrm{~B})$ & $112.9(10)$ \\
\hline \multirow[t]{2}{*}{$\mathrm{C}(25)-\mathrm{H}(25 \mathrm{C})$} & $0.942(18)$ & $\mathrm{H}(2 \mathrm{~A})-\mathrm{C}(2)-\mathrm{H}(2 \mathrm{~B})$ & $112.0(14)$ \\
\hline & & $\mathrm{N}(2)-\mathrm{C}(3)-\mathrm{C}(2)$ & $101.55(9)$ \\
\hline$C(16)-R u-C(1)$ & $92.60(5)$ & $\mathrm{N}(2)-\mathrm{C}(3)-\mathrm{H}(3 \mathrm{~A})$ & $109.4(8)$ \\
\hline $\mathrm{C}(16)-\mathrm{Ru}-\mathrm{O}(1)$ & $79.56(4)$ & $\mathrm{C}(2)-\mathrm{C}(3)-\mathrm{H}(3 \mathrm{~A})$ & $112.3(8)$ \\
\hline $\mathrm{C}(1)-\mathrm{Ru}-\mathrm{O}(1)$ & $171.85(4)$ & $\mathrm{N}(2)-\mathrm{C}(3)-\mathrm{H}(3 \mathrm{~B})$ & $109.4(10)$ \\
\hline $\mathrm{C}(16)-\mathrm{Ru}-\mathrm{Cl}(1)$ & $105.30(3)$ & $\mathrm{C}(2)-\mathrm{C}(3)-\mathrm{H}(3 \mathrm{~B})$ & $112.3(9)$ \\
\hline $\mathrm{C}(1)-\mathrm{Ru}-\mathrm{Cl}(1)$ & $97.81(3)$ & $\mathrm{H}(3 \mathrm{~A})-\mathrm{C}(3)-\mathrm{H}(3 \mathrm{~B})$ & $111.5(13)$ \\
\hline $\mathrm{O}(1)-\mathrm{Ru}-\mathrm{Cl}(1)$ & $86.52(2)$ & $C(5)-C(4)-C(9)$ & $115.60(10)$ \\
\hline $\mathrm{C}(16)-\mathrm{Ru}-\mathrm{Cl}(2)$ & $96.83(3)$ & $\mathrm{C}(5)-\mathrm{C}(4)-\mathrm{N}(1)$ & $122.39(10)$ \\
\hline $\mathrm{C}(1)-\mathrm{Ru}-\mathrm{Cl}(2)$ & $94.04(3)$ & $\mathrm{C}(9)-\mathrm{C}(4)-\mathrm{N}(1)$ & $121.96(10)$ \\
\hline $\mathrm{O}(1)-\mathrm{Ru}-\mathrm{Cl}(2)$ & $84.76(2)$ & $\mathrm{F}(1)-\mathrm{C}(5)-\mathrm{C}(6)$ & $118.83(11)$ \\
\hline $\mathrm{Cl}(1)-\mathrm{Ru}-\mathrm{Cl}(2)$ & $154.289(11)$ & $\mathrm{F}(1)-\mathrm{C}(5)-\mathrm{C}(4)$ & $117.69(11)$ \\
\hline $\mathrm{C}(22)-\mathrm{O}(1)-\mathrm{C}(23)$ & $120.70(9)$ & $C(6)-C(5)-C(4)$ & $123.48(11)$ \\
\hline $\mathrm{C}(22)-\mathrm{O}(1)-\mathrm{Ru}$ & 109.92(6) & $C(5)-C(6)-C(7)$ & $118.67(12)$ \\
\hline $\mathrm{C}(23)-\mathrm{O}(1)-\mathrm{Ru}$ & $129.33(7)$ & $\mathrm{C}(5)-\mathrm{C}(6)-\mathrm{H}(6)$ & $120.6(14)$ \\
\hline $\mathrm{C}(1)-\mathrm{N}(1)-\mathrm{C}(4)$ & $125.72(9)$ & $\mathrm{C}(7)-\mathrm{C}(6)-\mathrm{H}(6)$ & $120.7(14)$ \\
\hline $\mathrm{C}(1)-\mathrm{N}(1)-\mathrm{C}(2)$ & $113.01(9)$ & $C(8)-C(7)-C(6)$ & $120.31(12)$ \\
\hline $\mathrm{C}(4)-\mathrm{N}(1)-\mathrm{C}(2)$ & $121.24(9)$ & $\mathrm{C}(8)-\mathrm{C}(7)-\mathrm{H}(7)$ & $120.5(11)$ \\
\hline $\mathrm{C}(1)-\mathrm{N}(2)-\mathrm{C}(10)$ & $123.69(9)$ & $\mathrm{C}(6)-\mathrm{C}(7)-\mathrm{H}(7)$ & 119.1(11) \\
\hline $\mathrm{C}(1)-\mathrm{N}(2)-\mathrm{C}(3)$ & $112.65(9)$ & $\mathrm{C}(9)-\mathrm{C}(8)-\mathrm{C}(7)$ & $118.66(11)$ \\
\hline $\mathrm{C}(10)-\mathrm{N}(2)-\mathrm{C}(3)$ & $116.94(9)$ & $\mathrm{C}(9)-\mathrm{C}(8)-\mathrm{H}(8)$ & $115.7(12)$ \\
\hline $\mathrm{N}(1)-\mathrm{C}(1)-\mathrm{N}(2)$ & $105.61(9)$ & $\mathrm{C}(7)-\mathrm{C}(8)-\mathrm{H}(8)$ & $125.5(12)$ \\
\hline $\mathrm{N}(1)-\mathrm{C}(1)-\mathrm{Ru}$ & $126.41(8)$ & $\mathrm{F}(2)-\mathrm{C}(9)-\mathrm{C}(8)$ & $119.19(10)$ \\
\hline
\end{tabular}




\begin{tabular}{|c|c|c|c|}
\hline $\mathrm{F}(2)-\mathrm{C}(9)-\mathrm{C}(4)$ & $117.53(10)$ & $C(18)-C(19)-C(20)$ & $119.48(12)$ \\
\hline $\mathrm{C}(8)-\mathrm{C}(9)-\mathrm{C}(4)$ & $123.26(11)$ & $\mathrm{C}(18)-\mathrm{C}(19)-\mathrm{H}(19)$ & $119.7(10)$ \\
\hline$C(11)-C(10)-C(15)$ & $116.54(10)$ & $\mathrm{C}(20)-\mathrm{C}(19)-\mathrm{H}(19)$ & $120.8(10)$ \\
\hline $\mathrm{C}(11)-\mathrm{C}(10)-\mathrm{N}(2)$ & $121.27(10)$ & $C(19)-C(20)-C(21)$ & $121.71(11)$ \\
\hline $\mathrm{C}(15)-\mathrm{C}(10)-\mathrm{N}(2)$ & $122.01(10)$ & $\mathrm{C}(19)-\mathrm{C}(20)-\mathrm{H}(20)$ & $119.8(14)$ \\
\hline $\mathrm{F}(3)-\mathrm{C}(11)-\mathrm{C}(12)$ & $119.72(11)$ & $\mathrm{C}(21)-\mathrm{C}(20)-\mathrm{H}(20)$ & $118.5(14)$ \\
\hline $\mathrm{F}(3)-\mathrm{C}(11)-\mathrm{C}(10)$ & $117.73(10)$ & $C(22)-C(21)-C(20)$ & $118.40(12)$ \\
\hline$C(12)-C(11)-C(10)$ & $122.54(11)$ & $\mathrm{C}(22)-\mathrm{C}(21)-\mathrm{H}(21)$ & $119.4(11)$ \\
\hline$C(11)-C(12)-C(13)$ & $118.24(12)$ & $\mathrm{C}(20)-\mathrm{C}(21)-\mathrm{H}(21)$ & $122.2(11)$ \\
\hline $\mathrm{C}(11)-\mathrm{C}(12)-\mathrm{H}(12)$ & $120.1(11)$ & $\mathrm{O}(1)-\mathrm{C}(22)-\mathrm{C}(21)$ & $126.07(11)$ \\
\hline $\mathrm{C}(13)-\mathrm{C}(12)-\mathrm{H}(12)$ & $121.6(11)$ & $\mathrm{O}(1)-\mathrm{C}(22)-\mathrm{C}(17)$ & $113.08(9)$ \\
\hline$C(14)-C(13)-C(12)$ & $121.31(11)$ & $C(21)-C(22)-C(17)$ & $120.84(11)$ \\
\hline $\mathrm{C}(14)-\mathrm{C}(13)-\mathrm{H}(13)$ & $116.1(13)$ & $\mathrm{O}(1)-\mathrm{C}(23)-\mathrm{C}(25)$ & $106.47(10)$ \\
\hline $\mathrm{C}(12)-\mathrm{C}(13)-\mathrm{H}(13)$ & $122.4(13)$ & $\mathrm{O}(1)-\mathrm{C}(23)-\mathrm{C}(24)$ & $109.54(11)$ \\
\hline$C(15)-C(14)-C(13)$ & $118.19(12)$ & $C(25)-C(23)-C(24)$ & $112.07(12)$ \\
\hline $\mathrm{C}(15)-\mathrm{C}(14)-\mathrm{H}(14)$ & $118.4(9)$ & $\mathrm{O}(1)-\mathrm{C}(23)-\mathrm{H}(23)$ & $106.1(10)$ \\
\hline $\mathrm{C}(13)-\mathrm{C}(14)-\mathrm{H}(14)$ & $123.4(9)$ & $\mathrm{C}(25)-\mathrm{C}(23)-\mathrm{H}(23)$ & $110.4(10)$ \\
\hline $\mathrm{F}(4)-\mathrm{C}(15)-\mathrm{C}(14)$ & $119.19(11)$ & $\mathrm{C}(24)-\mathrm{C}(23)-\mathrm{H}(23)$ & $111.9(10)$ \\
\hline $\mathrm{F}(4)-\mathrm{C}(15)-\mathrm{C}(10)$ & $117.84(10)$ & $\mathrm{C}(23)-\mathrm{C}(24)-\mathrm{H}(24 \mathrm{~A})$ & $110.3(10)$ \\
\hline$C(14)-C(15)-C(10)$ & $122.96(11)$ & $\mathrm{C}(23)-\mathrm{C}(24)-\mathrm{H}(24 \mathrm{~B})$ & $104.8(10)$ \\
\hline $\mathrm{C}(17)-\mathrm{C}(16)-\mathrm{Ru}$ & $118.45(8)$ & $\mathrm{H}(24 \mathrm{~A})-\mathrm{C}(24)-\mathrm{H}(24 \mathrm{~B})$ & $105.6(15)$ \\
\hline $\mathrm{C}(17)-\mathrm{C}(16)-\mathrm{H}(16)$ & $114.7(10)$ & $\mathrm{C}(23)-\mathrm{C}(24)-\mathrm{H}(24 \mathrm{C})$ & $114.3(13)$ \\
\hline $\mathrm{Ru}-\mathrm{C}(16)-\mathrm{H}(16)$ & $126.5(10)$ & $\mathrm{H}(24 \mathrm{~A})-\mathrm{C}(24)-\mathrm{H}(24 \mathrm{C})$ & $111.8(17)$ \\
\hline$C(18)-C(17)-C(22)$ & $119.25(10)$ & $\mathrm{H}(24 \mathrm{~B})-\mathrm{C}(24)-\mathrm{H}(24 \mathrm{C})$ & $109.4(17)$ \\
\hline$C(18)-C(17)-C(16)$ & $122.44(10)$ & $\mathrm{C}(23)-\mathrm{C}(25)-\mathrm{H}(25 \mathrm{~A})$ & $111.2(10)$ \\
\hline$C(22)-C(17)-C(16)$ & $118.25(10)$ & $\mathrm{C}(23)-\mathrm{C}(25)-\mathrm{H}(25 \mathrm{~B})$ & $109.2(12)$ \\
\hline$C(19)-C(18)-C(17)$ & $120.23(12)$ & $\mathrm{H}(25 \mathrm{~A})-\mathrm{C}(25)-\mathrm{H}(25 \mathrm{~B})$ & $108.5(15)$ \\
\hline $\mathrm{C}(19)-\mathrm{C}(18)-\mathrm{H}(18)$ & 119.7(9) & $\mathrm{C}(23)-\mathrm{C}(25)-\mathrm{H}(25 \mathrm{C})$ & $109.0(11)$ \\
\hline $\mathrm{C}(17)-\mathrm{C}(18)-\mathrm{H}(18)$ & $120.1(9)$ & $\mathrm{H}(25 \mathrm{~A})-\mathrm{C}(25)-\mathrm{H}(25 \mathrm{C})$ & $109.1(15)$ \\
\hline
\end{tabular}


$\mathrm{H}(25 \mathrm{~B})-\mathrm{C}(25)-\mathrm{H}(25 \mathrm{C})$

109.8(16) 
Table 5. Anisotropic displacement parameters $\left(\AA^{2} \mathbf{x} 10^{4}\right)$ for CCDC 277100 . The anisotropic displacement factor exponent takes the form: $-2 \pi^{2}\left[h^{2} a^{* 2} U 11+\ldots+2 h \mathbf{k}^{*} \mathbf{b}^{*}\right.$ $\left.\mathbf{U}^{12}\right]$

\begin{tabular}{|c|c|c|c|c|c|c|}
\hline & $\mathrm{U}^{11}$ & $\mathrm{U}^{22}$ & $\mathrm{U}^{33}$ & $\mathrm{U}^{23}$ & $\mathrm{U}^{13}$ & $\mathrm{U}^{12}$ \\
\hline $\mathrm{Ru}$ & $79(1)$ & $68(1)$ & $74(1)$ & $2(1)$ & $2(1)$ & $0(1)$ \\
\hline $\mathrm{Cl}(1)$ & $153(1)$ & $107(1)$ & $120(1)$ & $16(1)$ & $-27(1)$ & $17(1)$ \\
\hline $\mathrm{Cl}(2)$ & $109(1)$ & $130(1)$ & $130(1)$ & $-3(1)$ & $-13(1)$ & $34(1)$ \\
\hline $\mathrm{F}(1)$ & $217(4)$ & $433(6)$ & $255(4)$ & $233(4)$ & $-134(3)$ & $-132(4)$ \\
\hline $\mathrm{F}(2)$ & $183(4)$ & $285(4)$ & 191(4) & $139(3)$ & $-37(3)$ & $-16(3)$ \\
\hline $\mathrm{F}(3)$ & $195(4)$ & $100(3)$ & $237(4)$ & $5(2)$ & $-9(3)$ & $-12(3)$ \\
\hline $\mathrm{F}(4)$ & $149(4)$ & $159(3)$ & $196(3)$ & $45(3)$ & $7(3)$ & $-36(3)$ \\
\hline $\mathrm{O}(1)$ & $140(4)$ & 119(3) & $98(3)$ & $4(2)$ & $38(3)$ & $-13(3)$ \\
\hline $\mathrm{N}(1)$ & $90(4)$ & $110(4)$ & $89(3)$ & $-14(3)$ & $-5(3)$ & $-11(3)$ \\
\hline $\mathrm{N}(2)$ & $86(4)$ & $116(4)$ & $95(3)$ & $-34(3)$ & $-4(3)$ & $-3(3)$ \\
\hline $\mathrm{C}(1)$ & $91(4)$ & $70(4)$ & $104(4)$ & $3(3)$ & $-11(3)$ & $-2(3)$ \\
\hline $\mathrm{C}(2)$ & $136(5)$ & $133(4)$ & $97(4)$ & $-31(3)$ & $-1(3)$ & $-6(4)$ \\
\hline$C(3)$ & $133(5)$ & $149(5)$ & $118(4)$ & $-52(3)$ & $11(4)$ & $-5(4)$ \\
\hline$C(4)$ & $97(4)$ & $87(4)$ & $116(4)$ & $-4(3)$ & $-14(3)$ & $-15(3)$ \\
\hline$C(5)$ & $149(5)$ & $167(5)$ & $136(5)$ & $48(4)$ & $-49(4)$ & $-44(4)$ \\
\hline$C(6)$ & $176(6)$ & $218(6)$ & $169(5)$ & $48(4)$ & $-14(4)$ & $-72(4)$ \\
\hline$C(7)$ & $120(5)$ & $190(5)$ & $190(5)$ & $-15(4)$ & $-4(4)$ & $-40(4)$ \\
\hline $\mathrm{C}(8)$ & $126(5)$ & $199(5)$ & $153(5)$ & $-3(4)$ & $-40(4)$ & $4(4)$ \\
\hline$C(9)$ & $133(5)$ & $142(5)$ & 112(4) & $24(3)$ & $-23(3)$ & $-3(4)$ \\
\hline$C(10)$ & $84(4)$ & $109(4)$ & $116(4)$ & $-23(3)$ & $1(3)$ & $-1(3)$ \\
\hline$C(11)$ & $123(5)$ & $117(4)$ & $164(5)$ & $-9(3)$ & $10(4)$ & $8(4)$ \\
\hline$C(12)$ & $150(6)$ & $180(5)$ & $184(5)$ & $6(4)$ & $-28(4)$ & $53(4)$ \\
\hline$C(13)$ & $128(5)$ & $241(6)$ & $201(6)$ & $-29(4)$ & $-57(4)$ & $22(4)$ \\
\hline$C(14)$ & $115(5)$ & $188(5)$ & $202(5)$ & $-44(4)$ & $-20(4)$ & $-35(4)$ \\
\hline
\end{tabular}




\begin{tabular}{lrrrrrr}
$\mathrm{C}(15)$ & $104(5)$ & $134(5)$ & $147(4)$ & $-11(3)$ & $7(3)$ & $-3(3)$ \\
$\mathrm{C}(16)$ & $108(5)$ & $85(4)$ & $90(4)$ & $-4(3)$ & $9(3)$ & $-4(3)$ \\
$\mathrm{C}(17)$ & $119(5)$ & $78(4)$ & $95(4)$ & $-7(3)$ & $-13(3)$ & $3(3)$ \\
$\mathrm{C}(18)$ & $179(6)$ & $104(4)$ & $139(5)$ & $-2(3)$ & $1(4)$ & $-16(4)$ \\
$\mathrm{C}(19)$ & $250(7)$ & $94(4)$ & $178(5)$ & $-31(4)$ & $-32(4)$ & $-23(4)$ \\
$\mathrm{C}(20)$ & $258(7)$ & $117(5)$ & $146(5)$ & $-56(4)$ & $-34(4)$ & $32(4)$ \\
$\mathrm{C}(21)$ & $172(6)$ & $144(5)$ & $107(4)$ & $-34(3)$ & $-4(4)$ & $39(4)$ \\
$\mathrm{C}(22)$ & $122(5)$ & $102(4)$ & $88(4)$ & $-8(3)$ & $-12(3)$ & $18(3)$ \\
$\mathrm{C}(23)$ & $144(6)$ & $211(6)$ & $127(5)$ & $42(4)$ & $64(4)$ & $35(4)$ \\
$\mathrm{C}(24)$ & $337(8)$ & $309(8)$ & $120(5)$ & $56(5)$ & $21(5)$ & $20(6)$ \\
$\mathrm{C}(25)$ & $173(6)$ & $215(6)$ & $282(7)$ & $64(5)$ & $80(5)$ & $-22(5)$ \\
& & & & & \\
\hline
\end{tabular}


Table 6. Hydrogen coordinates ( $\left.x 1^{4}\right)$ and isotropic displacement parameters $\left(\AA^{2} \times 10^{3}\right)$ for CCDC 277100.

\begin{tabular}{|c|c|c|c|c|}
\hline & $\mathrm{x}$ & $\mathrm{y}$ & $\mathrm{z}$ & $\mathrm{U}_{\text {iso }}$ \\
\hline $\mathrm{H}(2 \mathrm{~A})$ & $7648(11)$ & 9193(13) & $-550(15)$ & $22(4)$ \\
\hline $\mathrm{H}(2 \mathrm{~B})$ & $7594(10)$ & $8038(13)$ & $-372(14)$ & $18(4)$ \\
\hline $\mathrm{H}(3 \mathrm{~A})$ & $8869(10)$ & $7897(12)$ & $617(13)$ & $14(4)$ \\
\hline $\mathrm{H}(3 \mathrm{~B})$ & $9021(10)$ & $8961(12)$ & $-82(14)$ & $14(4)$ \\
\hline $\mathrm{H}(6)$ & $5151(14)$ & $7222(16)$ & $2843(19)$ & $44(6)$ \\
\hline $\mathrm{H}(7)$ & $4218(11)$ & $7679(13)$ & $1408(15)$ & $23(5)$ \\
\hline $\mathrm{H}(8)$ & $4646(12)$ & $8734(13)$ & $-140(15)$ & $26(5)$ \\
\hline $\mathrm{H}(12)$ & $10344(12)$ & $7880(14)$ & $4283(16)$ & $29(5)$ \\
\hline $\mathrm{H}(13)$ & $11194(12)$ & $9326(14)$ & $4190(16)$ & $29(5)$ \\
\hline $\mathrm{H}(14)$ & $10902(10)$ & $10646(13)$ & $2958(14)$ & $17(4)$ \\
\hline $\mathrm{H}(16)$ & $8389(10)$ & $11021(12)$ & 2601(13) & $12(4)$ \\
\hline $\mathrm{H}(18)$ & 8791(10) & $12729(12)$ & $3138(14)$ & $13(4)$ \\
\hline H(19) & $8478(10)$ & $14082(12)$ & $4342(13)$ & $10(4)$ \\
\hline $\mathrm{H}(20)$ & $7454(11)$ & $13924(13)$ & $5697(15)$ & $21(4)$ \\
\hline $\mathrm{H}(21)$ & $6721(11)$ & 12531(13) & $6146(15)$ & $23(4)$ \\
\hline $\mathrm{H}(23)$ & $5965(10)$ & $11117(12)$ & $5937(14)$ & $16(4)$ \\
\hline $\mathrm{H}(24 \mathrm{~A})$ & $7235(12)$ & 9995(14) & $7115(16)$ & $22(5)$ \\
\hline $\mathrm{H}(24 \mathrm{~B})$ & $6402(12)$ & $10411(15)$ & $7740(17)$ & $32(5)$ \\
\hline $\mathrm{H}(24 \mathrm{C})$ & $7102(13)$ & $11111(16)$ & $7297(17)$ & $36(6)$ \\
\hline $\mathrm{H}(25 \mathrm{~A})$ & $5708(11)$ & $9639(13)$ & $4836(15)$ & $18(4)$ \\
\hline $\mathrm{H}(25 \mathrm{~B})$ & $5477(13)$ & $9496(16)$ & $6234(18)$ & $44(6)$ \\
\hline $\mathrm{H}(25 \mathrm{C})$ & $6357(11)$ & $9123(14)$ & $5688(15)$ & $25(5)$ \\
\hline
\end{tabular}


Table 7. Torsion and dihedral angles $\left[{ }^{\circ}\right]$ for CCDC 277100.

$\begin{array}{lc}\mathrm{C}(16)-\mathrm{Ru}-\mathrm{C}(1)-\mathrm{N}(1) & -130.17(10) \\ \mathrm{C}(16)-\mathrm{Ru}-\mathrm{C}(1)-\mathrm{N}(2) & 38.59(10) \\ \mathrm{C}(1)-\mathrm{N}(1)-\mathrm{C}(4)-\mathrm{C}(5) & -50.49(16) \\ \mathrm{C}(11)-\mathrm{C}(10)-\mathrm{N}(2)-\mathrm{C}(1) & 70.12(15)\end{array}$

\section{$\underline{\text { Dihedrals }}$}

Plane 1

$\mathrm{C}(4)-\mathrm{N}(1)-\mathrm{C}(1)-\mathrm{N}(2)-\mathrm{C}(10)$

$\mathrm{C}(4)-\mathrm{N}(1)-\mathrm{C}(1)-\mathrm{N}(2)-\mathrm{C}(10)$

$\mathrm{C}(4)-\mathrm{N}(1)-\mathrm{C}(1)-\mathrm{N}(2)-\mathrm{C}(10)$
Plane 2

$\mathrm{Ru}-\mathrm{C}(16)>\mathrm{C}(22)-\mathrm{O}(1) \quad 50.88(2)$

$\mathrm{C}(4)>\mathrm{C}(9)-\mathrm{F}(1)-\mathrm{F}(2) \quad 64.39(2)$

$\mathrm{C}(10)>\mathrm{C}(15)-\mathrm{F}(3)-\mathrm{F}(4) \quad 88.76(2)$ 
Dichloro-ether derived ruthenium complex S6

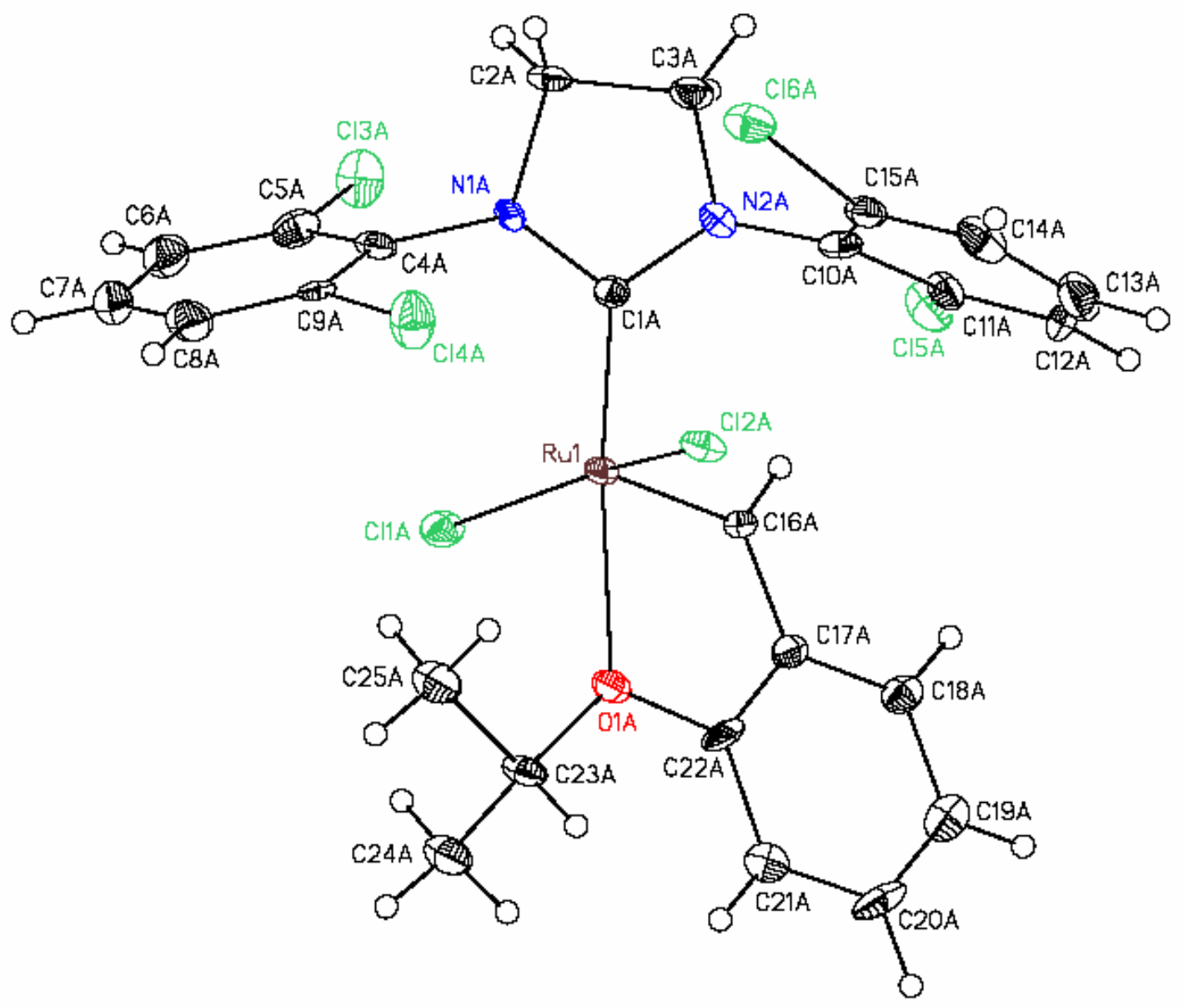




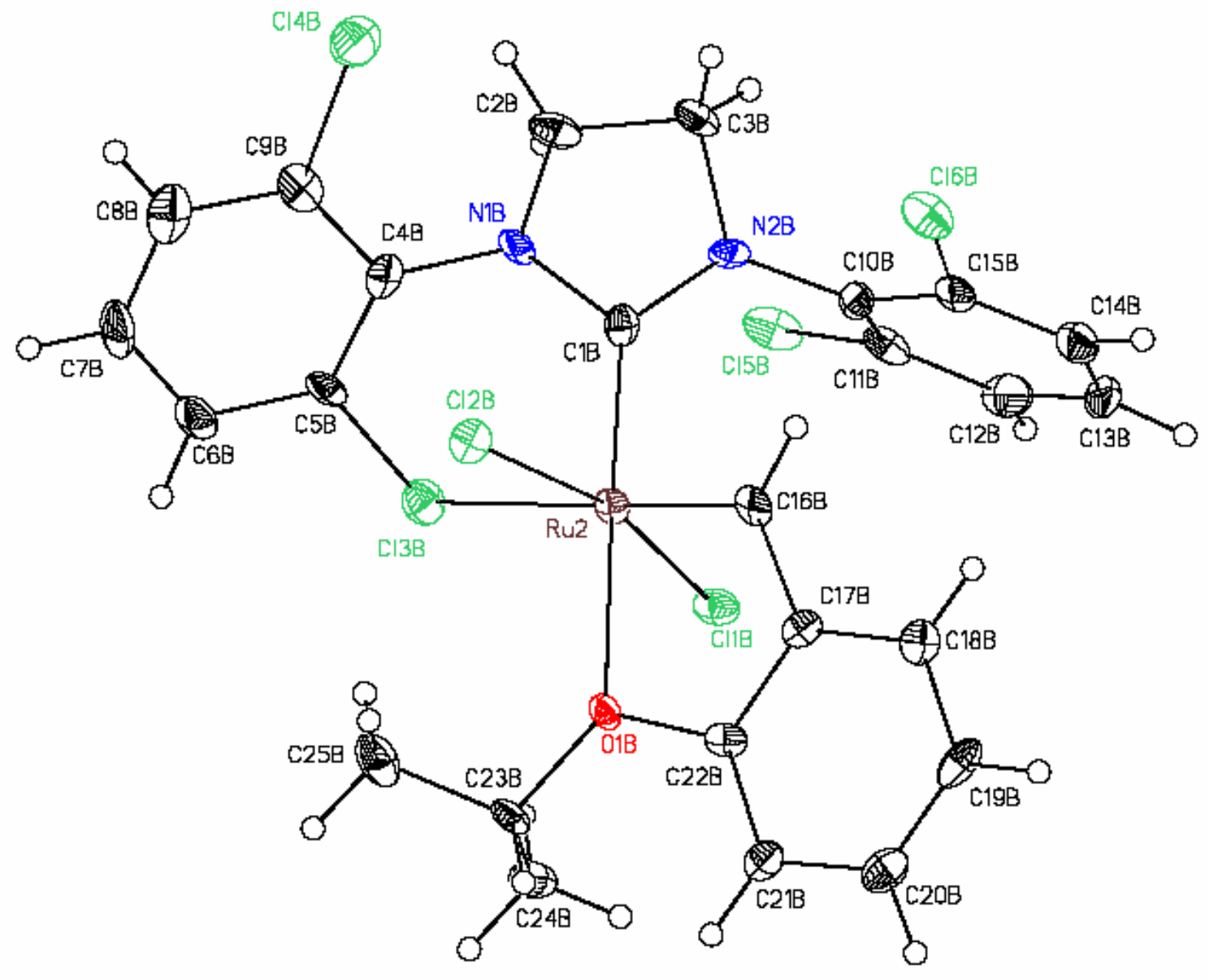


Table 1. Crystal data and structure refinement for CCDC 278852.

Empirical formula

Formula weight

Crystallization Solvent

Crystal Habit

Crystal size

Crystal color

\section{Data Collection}

Type of diffractometer

Wavelength

Data Collection Temperature

$\theta$ range for 30661 reflections used

in lattice determination

Unit cell dimensions

Volume

Z

Crystal system

Space group

Density (calculated)

$\mathrm{F}(000)$

Data collection program

$\theta$ range for data collection
$\mathrm{C}_{25} \mathrm{H}_{22} \mathrm{~N}_{2} \mathrm{OCl}_{6} \mathrm{Ru}$

680.22

THF/pentane

Fragment

$0.36 \times 0.33 \times 0.11 \mathrm{~mm}^{3}$

Green

Bruker SMART 1000

$0.71073 \AA \mathrm{MoK} \alpha$

100(2) K

2.48 to $32.55^{\circ}$

$a=15.9798(6) \AA$

$\mathrm{b}=16.9461(6) \AA$

$\beta=98.1850(10)^{\circ}$

$c=39.4746(15) \AA$

10580.6(7) $\AA^{3}$

16

Monoclinic

$\mathrm{P} 2{ }_{1} / \mathrm{c}$

$1.708 \mathrm{Mg} / \mathrm{m}^{3}$

5440

Bruker SMART v5.630

1.29 to $32.53^{\circ}$ 
Completeness to $\theta=32.53^{\circ}$

Index ranges

Data collection scan type

Data reduction program

Reflections collected

Independent reflections

Absorption coefficient

Absorption correction

Max. and min. transmission
$96.4 \%$

$-22 \leq \mathrm{h} \leq 24,-25 \leq \mathrm{k} \leq 25,-57 \leq 1 \leq 52$

$\omega$ scans at $5 \phi$ settings

Bruker SAINT v6.45A

157989

$37009\left[\mathrm{R}_{\mathrm{int}}=0.1459\right]$

$1.222 \mathrm{~mm}^{-1}$

None

0.8773 and 0.6674 


\section{Table 1 (cont.)}

\section{Structure solution and Refinement}

\begin{tabular}{ll} 
Structure solution program & Bruker XS v6.12 \\
Primary solution method & Direct methods \\
Secondary solution method & Difference Fourier map \\
Hydrogen placement & Geometric positions \\
Structure refinement program & Bruker XL v6.12 \\
Refinement method & Full matrix least-squares on F ${ }^{2}$ \\
Data / restraints / parameters & $37009 / 0 / 1261$ \\
Treatment of hydrogen atoms & Riding \\
Goodness-of-fit on F & 1.621 \\
Final R indices [I $>2 \sigma(\mathrm{I}), 20707$ reflections $]$ & $\mathrm{R} 1=0.1004, w \mathrm{R} 2=0.1215$ \\
R indices (all data) & $\mathrm{R} 1=0.1773, w \mathrm{R} 2=0.1363$ \\
Type of weighting scheme used & Sigma \\
Weighting scheme used & $w=1 / \sigma^{2}\left(\mathrm{Fo}^{2}\right)$ \\
Max shift/error & 0.002 \\
\hline argerage shift/error & 0.000 \\
\hline
\end{tabular}

\section{Special Refinement Details}

There are four molecules in the asymmetric unit, designated as molecules A, B, C and D. Molecules A and $\mathrm{C}$ are enantiomeric pairs as are molecules $\mathrm{B}$ and $\mathrm{D}$. Each molecule is related to its enantiomer by a noncrystallographic inversion center at $x=0.25, y=0.62, z=0.75$. In all four molecules the torsion angles (with respect to the imidazole ring) of the 2,6-dichlorophenyl rings labeled $\mathrm{C}\left(4^{*}\right)-\mathrm{C}\left(9^{*}\right)$ are significantly different than $90^{\circ}$, slightly more so for molecules B and D than for molecules A and C (see Table 6 and Figure 5). In molecules B and $\mathrm{D}$ one halogen on the ring is within bonding distance (although arguably weak) of Ru while in molecules $\mathrm{A}$ and $\mathrm{C}$ it is not (see Table 3 and Figure 6). The difference of the torsion angles of the isopropyl group (around $\mathrm{O}\left(1^{*}\right)$ $\mathrm{C}\left(23^{*}\right)$ ) between each pair may contribute to this (see Table 6 and Figure 5). In pair A,C one methyl adopts an "up" position which produces more steric crowding around the metal center than in pair B,D where neither methyl group is "up". Figure 6 shows another significant feature, the rotation by approximately $40^{\circ}$ of the imidazole ligand with 
respect to the plane of the 2-isoproxobenzylidene ligand.

The data was relatively weak throughout, with only $56 \%$ having $\mathrm{I}>2 \sigma(\mathrm{I})$. No correction was made for absorption and all large residual electron density peaks lie near the metal centers. Additionally the pattern of systematic absences/weakness in the data suggest the possibility of twinning or other crystal defect which was not possible to account for.

Refinement of $\mathrm{F}^{2}$ against ALL reflections. The weighted R-factor $(w \mathrm{R})$ and goodness of fit (S) are based on $\mathrm{F}^{2}$, conventional R-factors $(\mathrm{R})$ are based on $\mathrm{F}$, with $\mathrm{F}$ set to zero for negative $\mathrm{F}^{2}$. The threshold expression of $\mathrm{F}^{2}$ $>2 \sigma\left(\mathrm{F}^{2}\right)$ is used only for calculating R-factors(gt) etc. and is not relevant to the choice of reflections for refinement. R-factors based on $\mathrm{F}^{2}$ are statistically about twice as large as those based on $\mathrm{F}$, and R-factors based on ALL data will be even larger.

All esds (except the esd in the dihedral angle between two 1.s. planes) are estimated using the full covariance matrix. The cell esds are taken into account individually in the estimation of esds in distances, angles and torsion angles; correlations between esds in cell parameters are only used when they are defined by crystal symmetry. An approximate (isotropic) treatment of cell esds is used for estimating esds involving l.s. planes. 


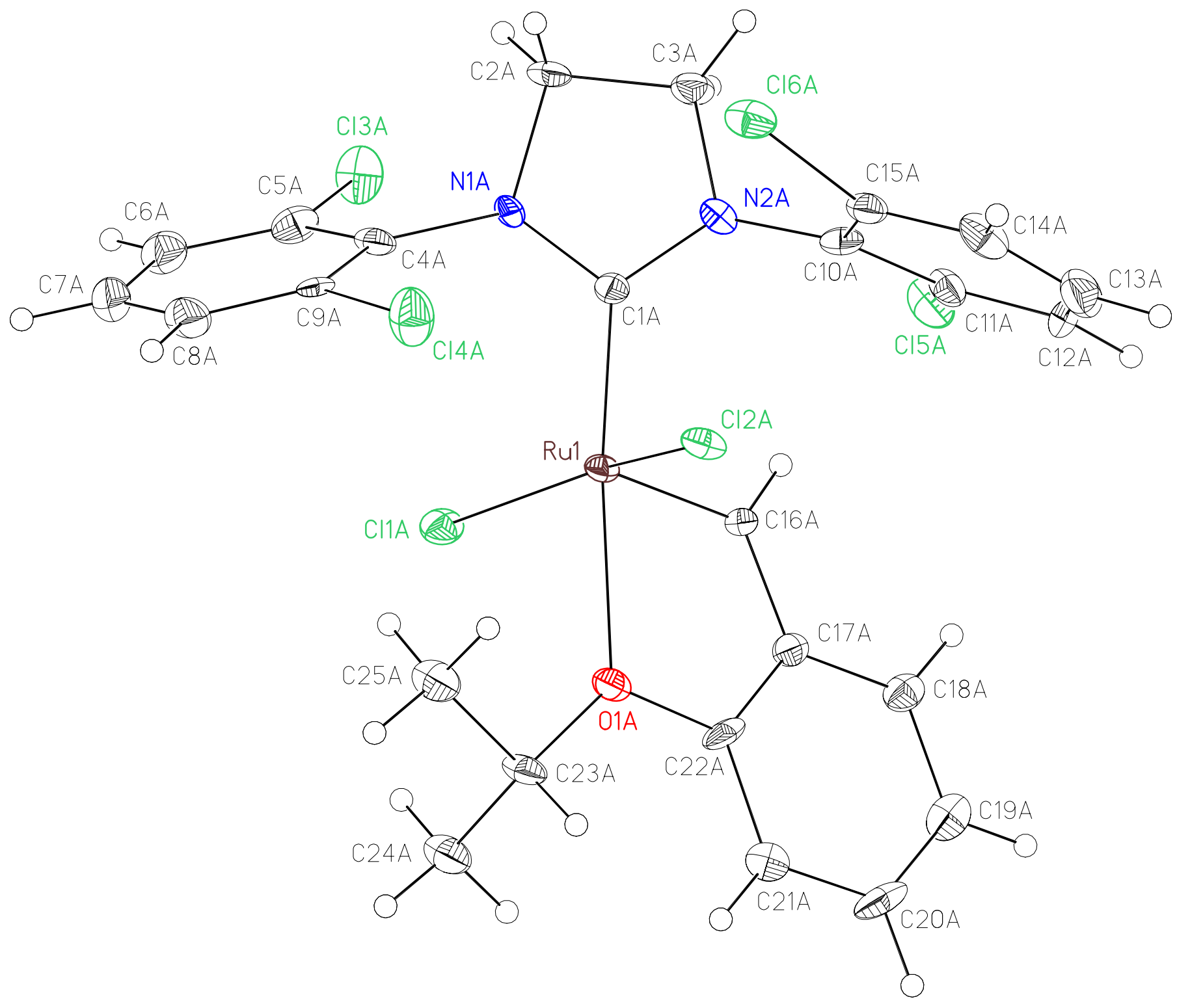




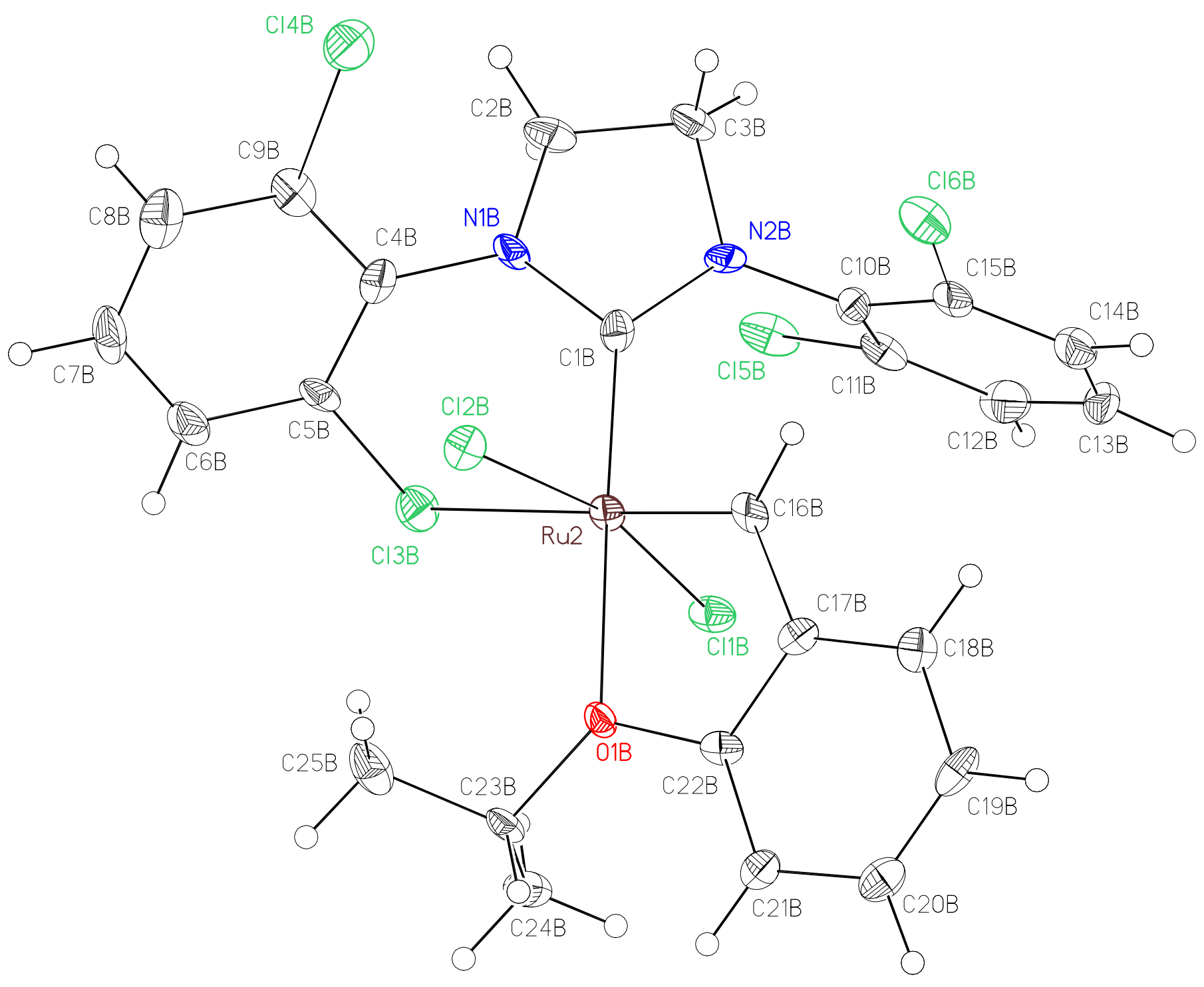




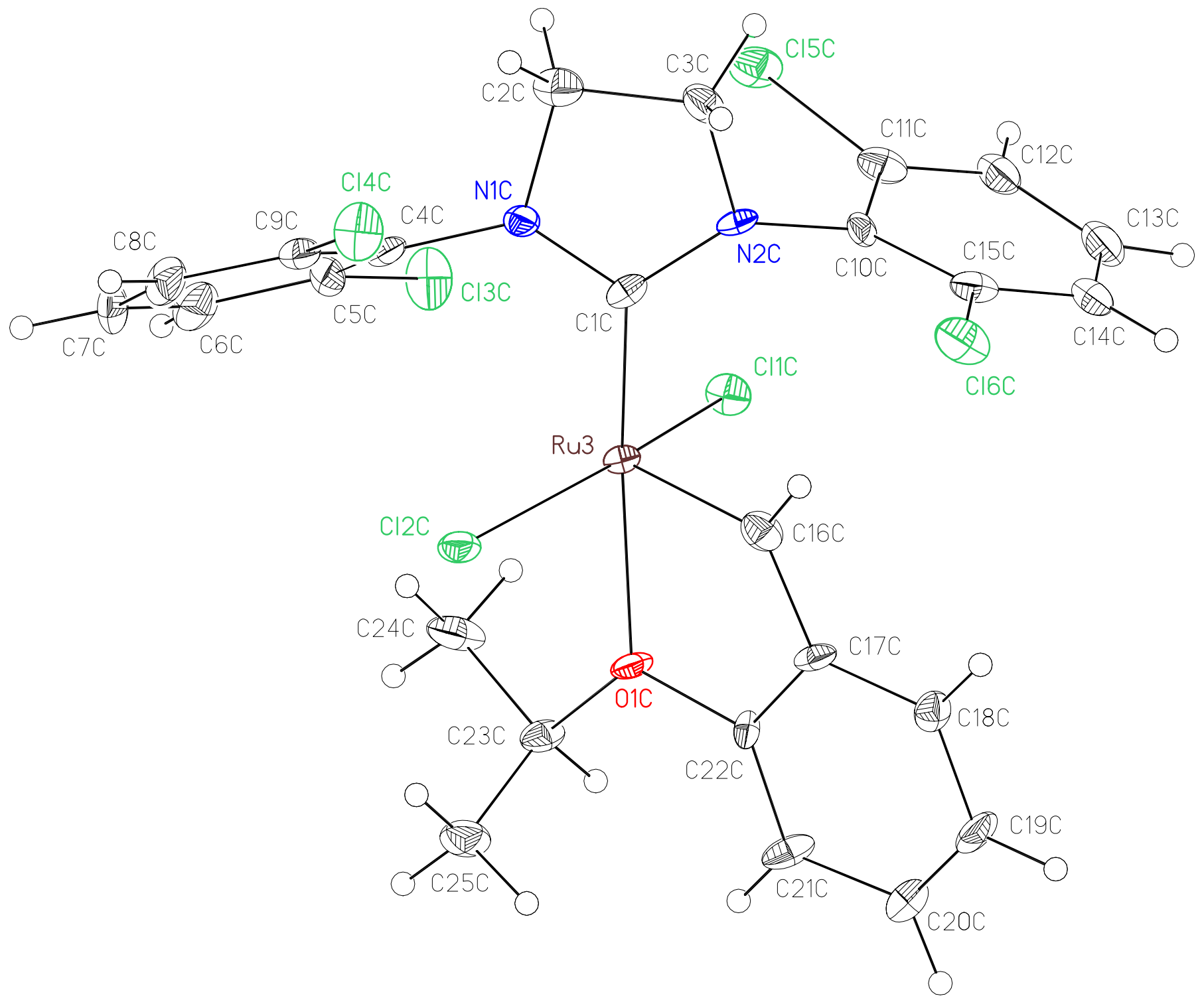




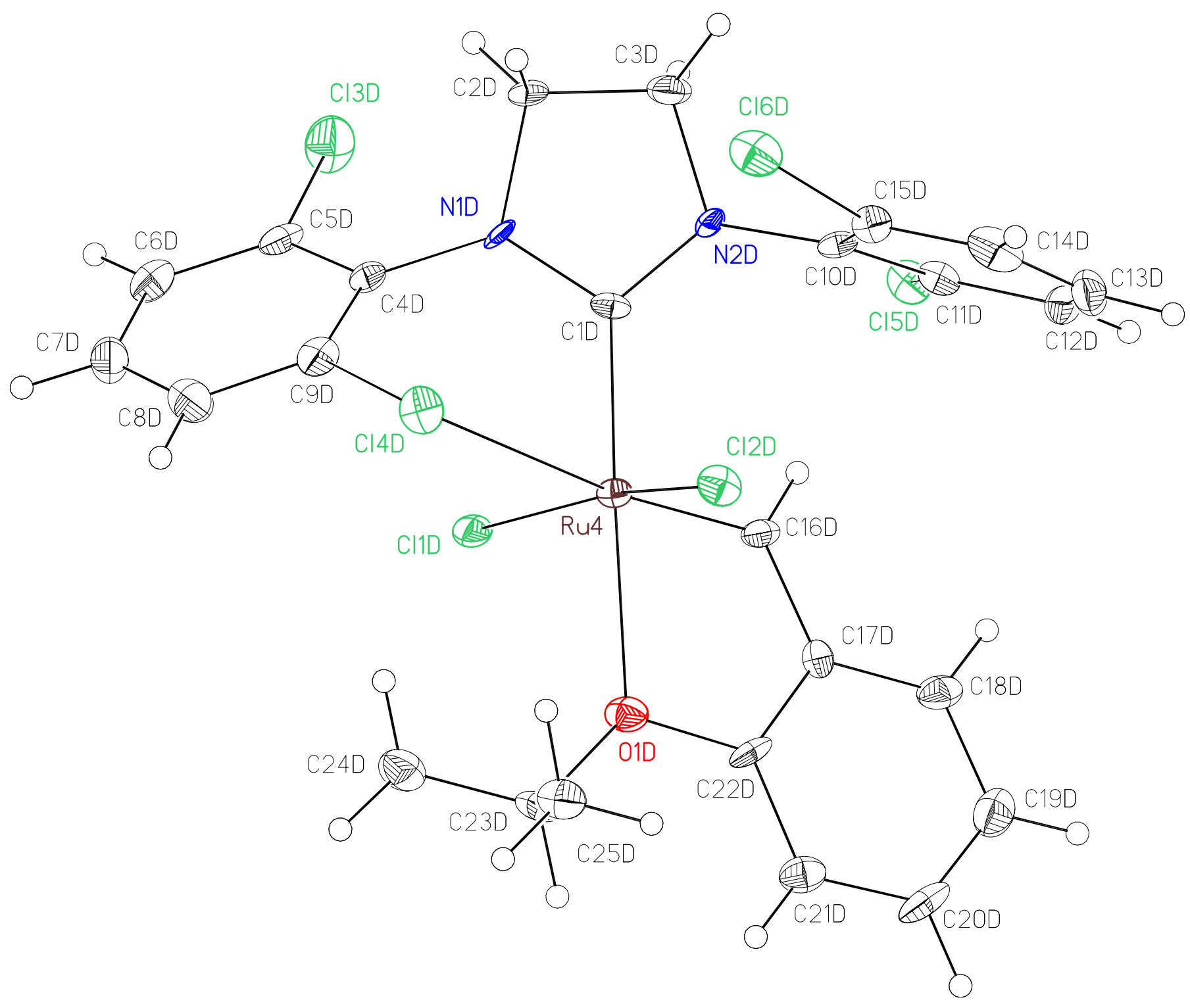




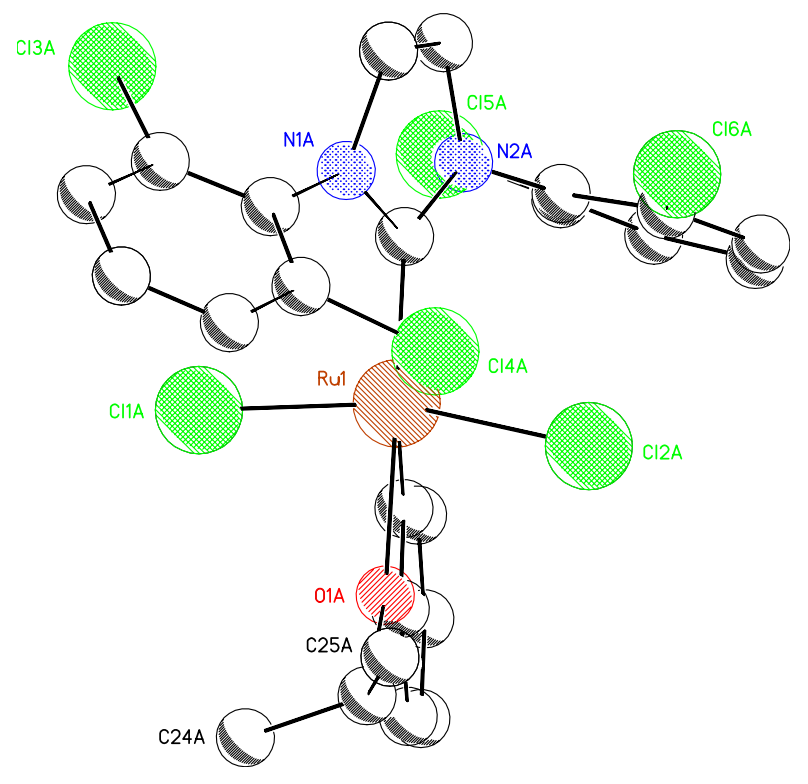

Molecule A

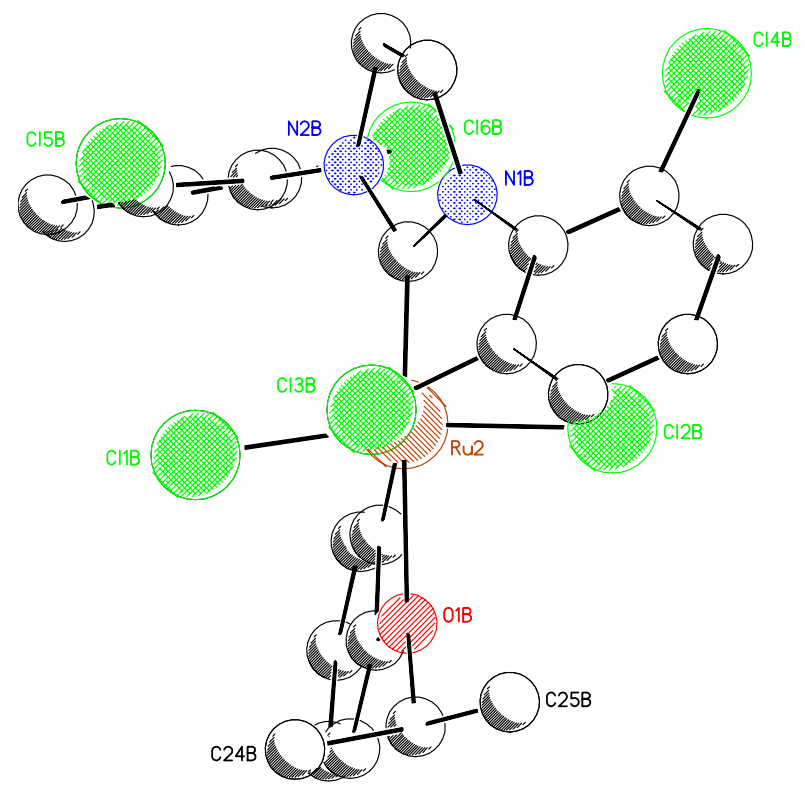

Molecule B

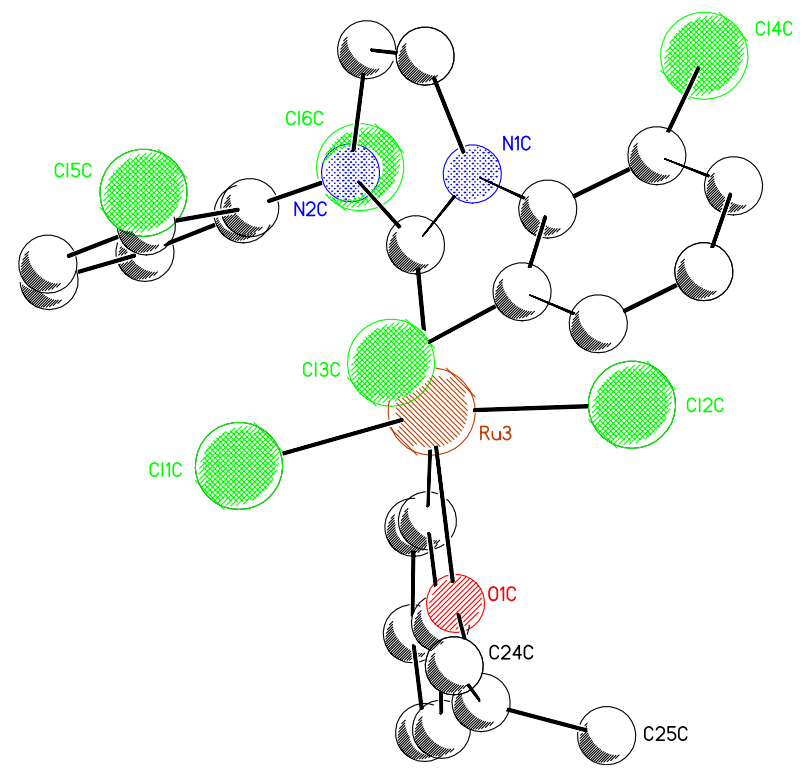

Molecule C

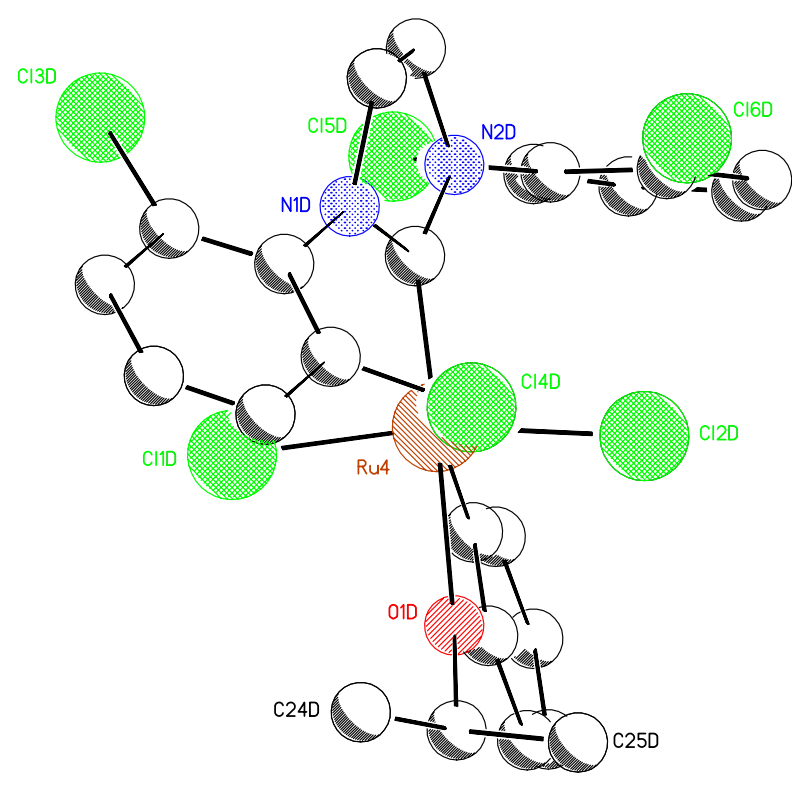

Molecule D 


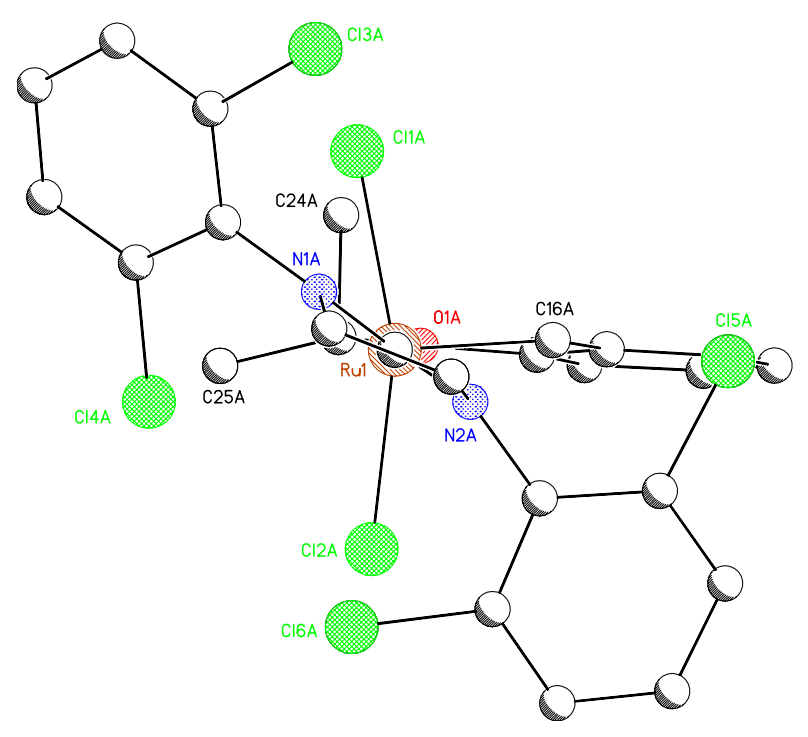

Molecule A

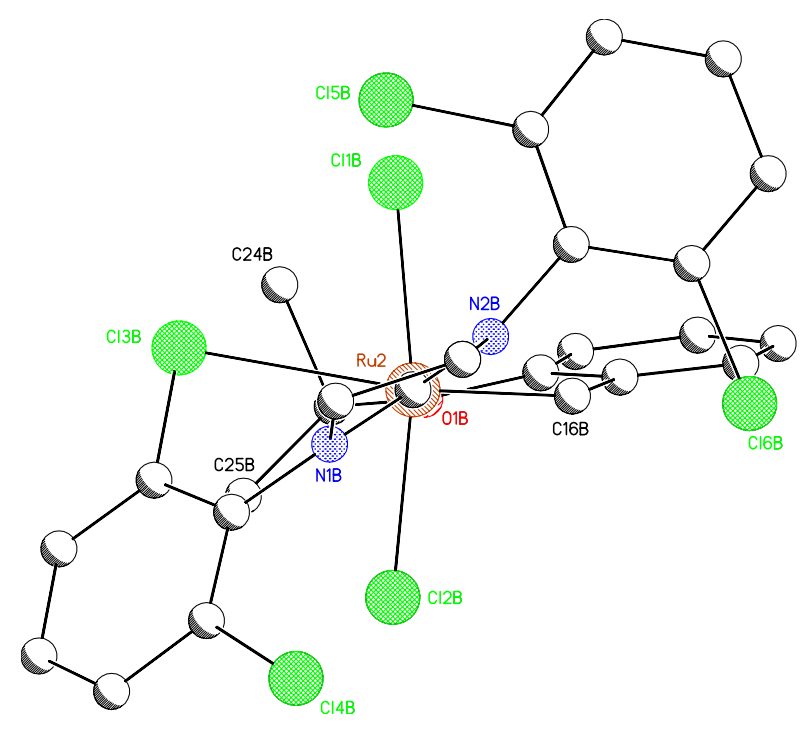

Molecule B

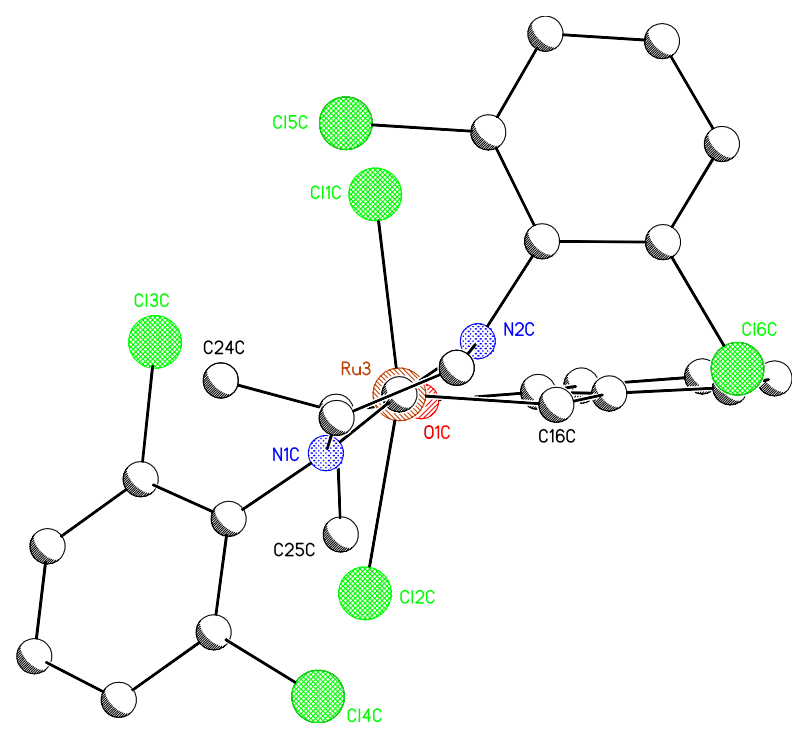

Molecule C

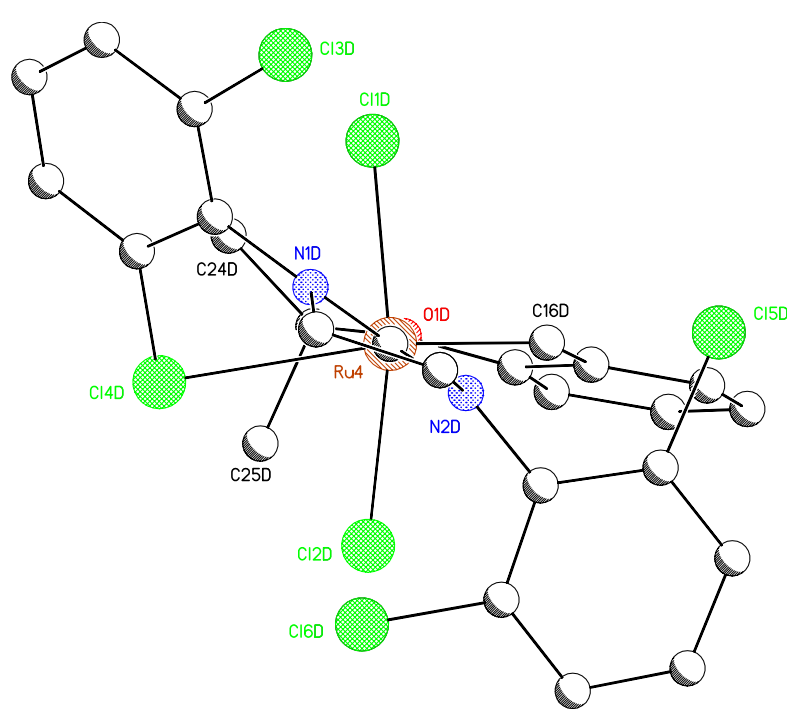

Molecule D 
Table 2. Atomic coordinates $\left(\mathrm{x} \mathrm{10}^{4}\right)$ and equivalent isotropic displacement parameters $\left(\AA^{2} \times 10^{3}\right)$ for CCDC 278852 . U(eq) is defined as the trace of the orthogonalized $U^{i j}$ tensor.

\begin{tabular}{|c|c|c|c|c|}
\hline & $\mathrm{x}$ & $\mathrm{y}$ & $\mathrm{z}$ & $\mathrm{U}_{\text {eq }}$ \\
\hline $\mathrm{Ru}(1)$ & $3790(1)$ & $4755(1)$ & $8742(1)$ & $12(1)$ \\
\hline $\mathrm{Cl}(1 \mathrm{~A})$ & $2708(1)$ & $4351(1)$ & $8313(1)$ & $17(1)$ \\
\hline $\mathrm{Cl}(2 \mathrm{~A})$ & $4796(1)$ & $5596(1)$ & $9048(1)$ & $16(1)$ \\
\hline $\mathrm{Cl}(3 \mathrm{~A})$ & $3755(1)$ & $2345(1)$ & $7892(1)$ & $26(1)$ \\
\hline $\mathrm{Cl}(4 \mathrm{~A})$ & $4890(1)$ & $5305(1)$ & $8222(1)$ & $27(1)$ \\
\hline $\mathrm{Cl}(5 \mathrm{~A})$ & $4198(1)$ & $2522(1)$ & $9452(1)$ & $24(1)$ \\
\hline $\mathrm{Cl}(6 \mathrm{~A})$ & $6504(1)$ & $4412(1)$ & $9020(1)$ & $20(1)$ \\
\hline $\mathrm{O}(1 \mathrm{~A})$ & $2854(2)$ & $5693(2)$ & $8876(1)$ & $14(1)$ \\
\hline $\mathrm{N}(1 \mathrm{~A})$ & 4672(3) & $3557(3)$ & $8364(1)$ & $12(1)$ \\
\hline $\mathrm{N}(2 \mathrm{~A})$ & 4988(3) & $3400(3)$ & 8911(1) & $14(1)$ \\
\hline$C(1 \mathrm{~A})$ & 4538(4) & $3862(3)$ & $8671(2)$ & $12(1)$ \\
\hline$C(2 A)$ & 5392(4) & 2994(4) & $8399(2)$ & $19(1)$ \\
\hline$C(3 \mathrm{~A})$ & $5425(4)$ & 2743(3) & $8770(2)$ & $16(1)$ \\
\hline$C(4 A)$ & 4330(4) & $3832(4)$ & $8029(2)$ & $16(1)$ \\
\hline$C(5 A)$ & 3919(4) & $3308(4)$ & $7782(2)$ & $18(1)$ \\
\hline$C(6 A)$ & 3598(4) & $3565(4)$ & $7460(2)$ & $21(1)$ \\
\hline$C(7 A)$ & $3673(4)$ & $4348(4)$ & $7371(2)$ & $25(2)$ \\
\hline$C(8 \mathrm{~A})$ & $4088(4)$ & $4879(4)$ & $7607(2)$ & $24(2)$ \\
\hline $\mathrm{C}(9 \mathrm{~A})$ & $4412(4)$ & $4612(3)$ & $7923(2)$ & $15(1)$ \\
\hline$C(10 A)$ & $5244(4)$ & $3631(3)$ & $9255(2)$ & $14(1)$ \\
\hline$C(11 \mathrm{~A})$ & 4901(4) & $3306(4)$ & $9532(2)$ & $15(1)$ \\
\hline$C(12 A)$ & $5121(4)$ & $3570(4)$ & $9863(2)$ & $21(2)$ \\
\hline$C(13 A)$ & $5752(5)$ & 4144(4) & 9931(2) & $26(2)$ \\
\hline$C(14 A)$ & 6154(4) & $4420(4)$ & $9672(2)$ & $21(2)$ \\
\hline$C(15 A)$ & 5918(4) & $4157(4)$ & $9340(2)$ & $16(1)$ \\
\hline
\end{tabular}




\begin{tabular}{|c|c|c|c|c|}
\hline$C(16 A)$ & $3368(4)$ & $4287(3)$ & $9099(2)$ & $12(1)$ \\
\hline$C(17 \mathrm{~A})$ & 2831(4) & 4721(3) & 9291(2) & $13(1)$ \\
\hline$C(18 A)$ & $2565(4)$ & $4440(4)$ & $9589(2)$ & $16(1)$ \\
\hline$C(19 A)$ & 2048(4) & $4885(4)$ & $9768(2)$ & $20(1)$ \\
\hline$C(20 A)$ & 1791(4) & $5632(4)$ & $9648(2)$ & $20(2)$ \\
\hline$C(21 \mathrm{~A})$ & 2042(4) & 5939(4) & $9352(2)$ & $18(1)$ \\
\hline$C(22 \mathrm{~A})$ & $2570(4)$ & $5473(4)$ & $9177(2)$ & $16(1)$ \\
\hline$C(23 \mathrm{~A})$ & $2540(4)$ & $6440(4)$ & $8707(2)$ & $16(1)$ \\
\hline$C(24 A)$ & 1649(4) & $6318(4)$ & $8521(2)$ & $22(1)$ \\
\hline$C(25 \mathrm{~A})$ & $3152(4)$ & $6647(4)$ & $8460(2)$ & $24(2)$ \\
\hline $\mathrm{Ru}(2)$ & $1355(1)$ & 2761(1) & $6285(1)$ & $12(1)$ \\
\hline $\mathrm{Cl}(1 \mathrm{~B})$ & $424(1)$ & $1847(1)$ & $5962(1)$ & $17(1)$ \\
\hline $\mathrm{Cl}(2 \mathrm{~B})$ & $2367(1)$ & $3413(1)$ & $6684(1)$ & $16(1)$ \\
\hline $\mathrm{Cl}(3 \mathrm{~B})$ & $525(1)$ & 2091(1) & $6774(1)$ & $20(1)$ \\
\hline $\mathrm{Cl}(4 \mathrm{~B})$ & $859(1)$ & $5159(1)$ & $7181(1)$ & $28(1)$ \\
\hline $\mathrm{Cl}(5 \mathrm{~B})$ & $-1397(1)$ & 2941(1) & $5949(1)$ & $23(1)$ \\
\hline $\mathrm{Cl}(6 \mathrm{~B})$ & $904(1)$ & 4998(1) & $5609(1)$ & $22(1)$ \\
\hline $\mathrm{O}(1 \mathrm{~B})$ & $2376(2)$ & $1860(2)$ & $6201(1)$ & $14(1)$ \\
\hline $\mathrm{N}(1 \mathrm{~B})$ & $340(3)$ & $3842(3)$ & $6659(1)$ & $14(1)$ \\
\hline $\mathrm{N}(2 \mathrm{~B})$ & $43(3)$ & 4023(3) & $6103(1)$ & $13(1)$ \\
\hline $\mathrm{C}(1 \mathrm{~B})$ & $534(4)$ & $3590(4)$ & $6346(2)$ & $13(1)$ \\
\hline $\mathrm{C}(2 \mathrm{~B})$ & $-451(4)$ & 4311(4) & $6615(2)$ & $21(1)$ \\
\hline$C(3 B)$ & $-487(4)$ & $4615(3)$ & $6253(2)$ & $19(1)$ \\
\hline $\mathrm{C}(4 \mathrm{~B})$ & $690(4)$ & $3589(4)$ & $6989(2)$ & $15(1)$ \\
\hline $\mathrm{C}(5 \mathrm{~B})$ & $817(4)$ & $2807(3)$ & $7083(2)$ & $15(1)$ \\
\hline $\mathrm{C}(6 \mathrm{~B})$ & 1177(4) & 2561(4) & $7406(2)$ & $20(1)$ \\
\hline$C(7 B)$ & 1422(4) & $3120(5)$ & $7655(2)$ & $27(2)$ \\
\hline $\mathrm{C}(8 \mathrm{~B})$ & 1301(4) & $3915(5)$ & $7579(2)$ & $25(2)$ \\
\hline
\end{tabular}




\begin{tabular}{|c|c|c|c|c|}
\hline $\mathrm{C}(9 \mathrm{~B})$ & $943(4)$ & 4133(4) & $7250(2)$ & 19(1) \\
\hline$C(10 B)$ & $-157(4)$ & $3830(4)$ & $5750(2)$ & $16(1)$ \\
\hline$C(11 B)$ & $-795(4)$ & $3300(4)$ & $5648(2)$ & $17(1)$ \\
\hline$C(12 B)$ & $-998(4)$ & $3079(4)$ & $5309(2)$ & $25(2)$ \\
\hline$C(13 B)$ & $-571(4)$ & $3422(4)$ & $5060(2)$ & $22(2)$ \\
\hline$C(14 B)$ & $23(4)$ & 4003(4) & $5157(2)$ & $22(2)$ \\
\hline$C(15 B)$ & $216(4)$ & 4212(4) & $5497(2)$ & $15(1)$ \\
\hline$C(16 B)$ & $1766(4)$ & $3184(3)$ & $5915(2)$ & $13(1)$ \\
\hline$C(17 B)$ & $2305(4)$ & 2704(3) & $5731(2)$ & $13(1)$ \\
\hline$C(18 B)$ & $2551(4)$ & 2933(4) & $5418(2)$ & $16(1)$ \\
\hline$C(19 B)$ & $3059(4)$ & $2440(4)$ & $5258(2)$ & $18(1)$ \\
\hline$C(20 B)$ & $3313(4)$ & 1712(4) & $5399(2)$ & $20(1)$ \\
\hline$C(21 B)$ & $3089(4)$ & $1478(4)$ & $5715(2)$ & $17(1)$ \\
\hline$C(22 B)$ & $2599(4)$ & 1988(3) & $5876(2)$ & $13(1)$ \\
\hline$C(23 B)$ & $2686(4)$ & 1153(3) & $6394(2)$ & $16(1)$ \\
\hline$C(24 B)$ & $2058(4)$ & 491(3) & $6304(2)$ & $22(1)$ \\
\hline$C(25 B)$ & $2804(4)$ & 1362(4) & $6765(2)$ & $23(1)$ \\
\hline $\mathrm{Ru}(3)$ & $1213(1)$ & $7648(1)$ & $6239(1)$ & $10(1)$ \\
\hline $\mathrm{Cl}(1 \mathrm{C})$ & $209(1)$ & 6792(1) & $5934(1)$ & $16(1)$ \\
\hline $\mathrm{Cl}(2 \mathrm{C})$ & $2280(1)$ & $8090(1)$ & $6664(1)$ & $18(1)$ \\
\hline $\mathrm{Cl}(3 \mathrm{C})$ & $94(1)$ & $7132(1)$ & $6751(1)$ & $26(1)$ \\
\hline $\mathrm{Cl}(4 \mathrm{C})$ & $1235(1)$ & 10091(1) & 7081(1) & $25(1)$ \\
\hline $\mathrm{Cl}(5 \mathrm{C})$ & $-1491(1)$ & 7998(1) & $5984(1)$ & $21(1)$ \\
\hline $\mathrm{Cl}(6 \mathrm{C})$ & $802(1)$ & $9787(1)$ & $5477(1)$ & $25(1)$ \\
\hline $\mathrm{O}(1 \mathrm{C})$ & 2154(2) & $6695(2)$ & $6128(1)$ & $15(1)$ \\
\hline $\mathrm{N}(1 \mathrm{C})$ & $330(3)$ & $8868(3)$ & $6604(1)$ & $14(1)$ \\
\hline $\mathrm{N}(2 \mathrm{C})$ & $32(3)$ & 9003(3) & $6048(1)$ & $12(1)$ \\
\hline$C(1 C)$ & $475(4)$ & $8540(3)$ & $6302(2)$ & $10(1)$ \\
\hline
\end{tabular}




\begin{tabular}{|c|c|c|c|c|}
\hline$C(2 C)$ & $-371(4)$ & $9445(4)$ & $6558(2)$ & $20(1)$ \\
\hline$C(3 C)$ & $-405(4)$ & $9669(4)$ & $6189(2)$ & $18(1)$ \\
\hline$C(4 C)$ & $641(4)$ & $8589(3)$ & $6935(2)$ & $13(1)$ \\
\hline$C(5 C)$ & $557(4)$ & 7817(4) & $7045(2)$ & $16(1)$ \\
\hline$C(6 C)$ & $870(4)$ & 7565(4) & $7372(2)$ & $23(1)$ \\
\hline$C(7 C)$ & $1259(4)$ & $8083(4)$ & $7610(2)$ & $20(1)$ \\
\hline$C(8 C)$ & $1349(4)$ & $8876(4)$ & $7515(2)$ & $23(2)$ \\
\hline$C(9 C)$ & 1045(4) & 9112(4) & $7184(2)$ & $16(1)$ \\
\hline$C(10 C)$ & $-254(4)$ & $8732(3)$ & $5710(2)$ & $11(1)$ \\
\hline$C(11 C)$ & $-948(4)$ & $8225(3)$ & $5646(2)$ & $17(1)$ \\
\hline$C(12 C)$ & $-1223(4)$ & 7926(4) & $5327(2)$ & $18(1)$ \\
\hline$C(13 C)$ & $-825(4)$ & 8161(4) & $5056(2)$ & $23(2)$ \\
\hline$C(14 C)$ & $-191(4)$ & $8725(4)$ & $5099(2)$ & $22(2)$ \\
\hline$C(15 C)$ & $72(4)$ & $9020(4)$ & $5422(2)$ & 19(1) \\
\hline$C(16 C)$ & 1654(4) & $8090(3)$ & $5879(2)$ & $14(1)$ \\
\hline$C(17 C)$ & $2200(4)$ & 7616(3) & $5696(2)$ & $13(1)$ \\
\hline$C(18 C)$ & 2481(4) & 7886(4) & $5393(2)$ & $14(1)$ \\
\hline$C(19 C)$ & $3011(4)$ & 7414(4) & $5235(2)$ & $20(1)$ \\
\hline$C(20 C)$ & $3266(4)$ & 6684(4) & $5369(2)$ & $19(1)$ \\
\hline$C(21 C)$ & $3008(4)$ & $6407(3)$ & $5671(2)$ & $18(1)$ \\
\hline$C(22 C)$ & $2468(4)$ & 6882(3) & $5827(2)$ & $11(1)$ \\
\hline$C(23 C)$ & $2452(4)$ & $5968(3)$ & $6313(2)$ & $14(1)$ \\
\hline$C(24 C)$ & $1824(4)$ & $5785(4)$ & $6547(2)$ & $24(2)$ \\
\hline$C(25 C)$ & $3333(4)$ & 6106(4) & $6498(2)$ & $22(1)$ \\
\hline $\mathrm{Ru}(4)$ & $3643(1)$ & 9651(1) & $8686(1)$ & $10(1)$ \\
\hline $\mathrm{Cl}(1 \mathrm{D})$ & $2620(1)$ & $9006(1)$ & $8293(1)$ & $16(1)$ \\
\hline $\mathrm{Cl}(2 \mathrm{D})$ & $4577(1)$ & $10574(1)$ & 8994(1) & $17(1)$ \\
\hline Cl(3D) & $4060(1)$ & 7241(1) & 7803(1) & $29(1)$ \\
\hline
\end{tabular}




\begin{tabular}{|c|c|c|c|c|}
\hline $\mathrm{Cl}(4 \mathrm{D})$ & $4466(1)$ & $10314(1)$ & $8186(1)$ & $20(1)$ \\
\hline $\mathrm{Cl}(5 \mathrm{D})$ & $4107(1)$ & $7427(1)$ & $9363(1)$ & $18(1)$ \\
\hline $\mathrm{Cl}(6 \mathrm{D})$ & $6388(1)$ & $9459(1)$ & $9008(1)$ & $23(1)$ \\
\hline $\mathrm{O}(1 \mathrm{D})$ & $2619(3)$ & $10546(2)$ & $8780(1)$ & $15(1)$ \\
\hline $\mathrm{N}(1 \mathrm{D})$ & $4638(3)$ & $8550(3)$ & $8311(1)$ & $12(1)$ \\
\hline $\mathrm{N}(2 \mathrm{D})$ & 4949(3) & $8379(3)$ & $8861(1)$ & 11(1) \\
\hline$C(1 D)$ & $4469(3)$ & $8817(3)$ & $8620(2)$ & $10(1)$ \\
\hline$C(2 D)$ & $5430(4)$ & $8090(4)$ & $8349(2)$ & $20(1)$ \\
\hline$C(3 D)$ & $5472(4)$ & 7787(4) & $8713(2)$ & 19(1) \\
\hline$C(4 D)$ & $4262(4)$ & 8796(3) & $7975(2)$ & $12(1)$ \\
\hline$C(5 D)$ & $3994(4)$ & $8243(3)$ & $7720(2)$ & $17(1)$ \\
\hline$C(6 D)$ & $3631(4)$ & $8473(4)$ & $7394(2)$ & $18(1)$ \\
\hline$C(7 D)$ & $3530(4)$ & $9257(4)$ & $7318(2)$ & $22(1)$ \\
\hline$C(8 D)$ & $3782(4)$ & 9824(4) & $7555(2)$ & $22(1)$ \\
\hline C(9D) & $4162(3)$ & 9593(4) & $7880(2)$ & $15(1)$ \\
\hline$C(10 D)$ & $5158(3)$ & 8593(3) & $9210(2)$ & $10(1)$ \\
\hline$C(11 D)$ & $4785(4)$ & $8218(3)$ & $9468(2)$ & $15(1)$ \\
\hline$C(12 D)$ & $4963(4)$ & $8453(4)$ & $9807(2)$ & $17(1)$ \\
\hline$C(13 D)$ & $5555(4)$ & 9046(4) & $9893(2)$ & $21(1)$ \\
\hline$C(14 D)$ & $5986(4)$ & 9383(4) & $9654(2)$ & $23(2)$ \\
\hline$C(15 D)$ & $5791(4)$ & $9145(3)$ & $9312(2)$ & $14(1)$ \\
\hline$C(16 D)$ & $3261(4)$ & $9235(3)$ & $9062(2)$ & $13(1)$ \\
\hline$C(17 D)$ & $2751(4)$ & 9721(3) & $9250(2)$ & $13(1)$ \\
\hline$C(18 D)$ & $2544(4)$ & 9524(4) & $9568(2)$ & $18(1)$ \\
\hline$C(19 D)$ & 2071(4) & $10015(4)$ & $9749(2)$ & $23(2)$ \\
\hline$C(20 D)$ & $1784(4)$ & $10717(4)$ & $9599(2)$ & $22(2)$ \\
\hline$C(21 D)$ & $1970(4)$ & $10950(3)$ & $9279(2)$ & $16(1)$ \\
\hline$C(22 D)$ & $2443(4)$ & $10438(3)$ & $9109(2)$ & $13(1)$ \\
\hline C(23D) & 2284(4) & 11253(3) & $8580(2)$ & $18(1)$ \\
\hline
\end{tabular}




\begin{tabular}{lllll}
$\mathrm{C}(24 \mathrm{D})$ & $2162(4)$ & $11024(4)$ & $8208(2)$ & $22(1)$ \\
$\mathrm{C}(25 \mathrm{D})$ & $2893(4)$ & $11936(4)$ & $8664(2)$ & $20(1)$ \\
& & & & \\
\hline
\end{tabular}


Table 3. Selected bond lengths $[\AA \AA]$ and angles $\left[^{\circ}\right]$ for CCDC 278852.

\begin{tabular}{|c|c|c|c|}
\hline $\mathrm{Ru}(1)-\mathrm{C}(16 \mathrm{~A})$ & $1.827(6)$ & $\operatorname{Ru}(3)-C(16 C)$ & $1.834(6)$ \\
\hline $\mathrm{Ru}(1)-\mathrm{C}(1 \mathrm{~A})$ & $1.972(6)$ & $\mathrm{Ru}(3)-\mathrm{C}(1 \mathrm{C})$ & $1.956(6)$ \\
\hline $\mathrm{Ru}(1)-\mathrm{O}(1 \mathrm{~A})$ & $2.297(4)$ & $\mathrm{Ru}(3)-\mathrm{O}(1 \mathrm{C})$ & $2.290(4)$ \\
\hline $\mathrm{Ru}(1)-\mathrm{Cl}(1 \mathrm{~A})$ & $2.3434(16)$ & $\mathrm{Ru}(3)-\mathrm{Cl}(2 \mathrm{C})$ & $2.3394(15)$ \\
\hline $\mathrm{Ru}(1)-\mathrm{Cl}(2 \mathrm{~A})$ & $2.3506(16)$ & $\mathrm{Ru}(3)-\mathrm{Cl}(1 \mathrm{C})$ & $2.3607(15)$ \\
\hline$* * \mathrm{Ru}(1)-\mathrm{Cl}(4 \mathrm{~A})$ & $3.0308(19)$ & $* * \operatorname{Ru}(3)-\mathrm{Cl}(3 \mathrm{C})$ & $3.0124(18)$ \\
\hline $\mathrm{Ru}(2)-\mathrm{C}(16 \mathrm{~B})$ & $1.829(6)$ & $\mathrm{Ru}(4)-\mathrm{C}(16 \mathrm{D})$ & $1.825(6)$ \\
\hline $\mathrm{Ru}(2)-\mathrm{C}(1 \mathrm{~B})$ & $1.962(6)$ & $\mathrm{Ru}(4)-\mathrm{C}(1 \mathrm{D})$ & $1.975(5)$ \\
\hline $\mathrm{Ru}(2)-\mathrm{O}(1 \mathrm{~B})$ & $2.291(4)$ & $\mathrm{Ru}(4)-\mathrm{O}(1 \mathrm{D})$ & $2.300(4)$ \\
\hline $\mathrm{Ru}(2)-\mathrm{Cl}(2 \mathrm{~B})$ & $2.3663(16)$ & $\mathrm{Ru}(4)-\mathrm{Cl}(1 \mathrm{D})$ & $2.3566(15)$ \\
\hline $\mathrm{Ru}(2)-\mathrm{Cl}(1 \mathrm{~B})$ & $2.3867(16)$ & $\mathrm{Ru}(4)-\mathrm{Cl}(2 \mathrm{D})$ & $2.3741(15)$ \\
\hline $\mathrm{Ru}(2)-\mathrm{Cl}(3 \mathrm{~B})$ & $2.7406(16)$ & $\mathrm{Ru}(4)-\mathrm{Cl}(4 \mathrm{D})$ & $2.7614(17)$ \\
\hline
\end{tabular}

$\begin{array}{lccc}\mathrm{C}(16 \mathrm{~A})-\mathrm{Ru}(1)-\mathrm{C}(1 \mathrm{~A}) & 94.3(2) & \mathrm{C}(16 \mathrm{~B})-\mathrm{Ru}(2)-\mathrm{C}(1 \mathrm{~B}) & 98.0(3) \\ \mathrm{C}(16 \mathrm{~A})-\mathrm{Ru}(1)-\mathrm{O}(1 \mathrm{~A}) & 78.3(2) & \mathrm{C}(16 \mathrm{~B})-\mathrm{Ru}(2)-\mathrm{O}(1 \mathrm{~B}) & 78.7(2) \\ \mathrm{C}(1 \mathrm{~A})-\mathrm{Ru}(1)-\mathrm{O}(1 \mathrm{~A}) & 172.6(2) & \mathrm{C}(1 \mathrm{~B})-\mathrm{Ru}(2)-\mathrm{O}(1 \mathrm{~B}) & 175.9(2) \\ \mathrm{C}(16 \mathrm{~A})-\mathrm{Ru}(1)-\mathrm{Cl}(1 \mathrm{~A}) & 96.61(19) & \mathrm{C}(16 \mathrm{~B})-\mathrm{Ru}(2)-\mathrm{Cl}(2 \mathrm{~B}) & 93.43(18) \\ \mathrm{C}(1 \mathrm{~A})-\mathrm{Ru}(1)-\mathrm{Cl}(1 \mathrm{~A}) & 94.20(18) & \mathrm{C}(1 \mathrm{~B})-\mathrm{Ru}(2)-\mathrm{Cl}(2 \mathrm{~B}) & 89.33(19) \\ \mathrm{O}(1 \mathrm{~A})-\mathrm{Ru}(1)-\mathrm{Cl}(1 \mathrm{~A}) & 86.08(11) & \mathrm{O}(1 \mathrm{~B})-\mathrm{Ru}(2)-\mathrm{Cl}(2 \mathrm{~B}) & 88.51(11) \\ \mathrm{C}(16 \mathrm{~A})-\mathrm{Ru}(1)-\mathrm{Cl}(2 \mathrm{~A}) & 99.59(19) & \mathrm{C}(16 \mathrm{~B})-\mathrm{Ru}(2)-\mathrm{Cl}(1 \mathrm{~B}) & 95.47(19) \\ \mathrm{C}(1 \mathrm{~A})-\mathrm{Ru}(1)-\mathrm{Cl}(2 \mathrm{~A}) & 98.87(18) & \mathrm{C}(1 \mathrm{~B})-\mathrm{Ru}(2)-\mathrm{Cl}(1 \mathrm{~B}) & 98.87(19) \\ \mathrm{O}(1 \mathrm{~A})-\mathrm{Ru}(1)-\mathrm{Cl}(2 \mathrm{~A}) & 83.19(11) & \mathrm{O}(1 \mathrm{~B})-\mathrm{Ru}(2)-\mathrm{Cl}(1 \mathrm{~B}) & 83.88(11) \\ \mathrm{Cl}(1 \mathrm{~A})-\mathrm{Ru}(1)-\mathrm{Cl}(2 \mathrm{~A}) & 158.30(6) & \mathrm{Cl}(2 \mathrm{~B})-\mathrm{Ru}(2)-\mathrm{Cl}(1 \mathrm{~B}) & 166.92(6) \\ & & \mathrm{C}(16 \mathrm{~B})-\mathrm{Ru}(2)-\mathrm{Cl}(3 \mathrm{~B}) & 171.59(18)\end{array}$




$\begin{array}{lccc}\mathrm{C}(1 \mathrm{~B})-\mathrm{Ru}(2)-\mathrm{Cl}(3 \mathrm{~B}) & 79.05(18) & \mathrm{C}(16 \mathrm{D})-\mathrm{Ru}(4)-\mathrm{C}(1 \mathrm{D}) & 97.9(2) \\ \mathrm{O}(1 \mathrm{~B})-\mathrm{Ru}(2)-\mathrm{Cl}(3 \mathrm{~B}) & 104.53(11) & \mathrm{C}(16 \mathrm{D})-\mathrm{Ru}(4)-\mathrm{O}(1 \mathrm{D}) & 78.2(2) \\ \mathrm{Cl}(2 \mathrm{~B})-\mathrm{Ru}(2)-\mathrm{Cl}(3 \mathrm{~B}) & 94.42(5) & \mathrm{C}(1 \mathrm{D})-\mathrm{Ru}(4)-\mathrm{O}(1 \mathrm{D}) & 175.4(2) \\ \mathrm{Cl}(1 \mathrm{~B})-\mathrm{Ru}(2)-\mathrm{Cl}(3 \mathrm{~B}) & 77.32(5) & \mathrm{C}(16 \mathrm{D})-\mathrm{Ru}(4)-\mathrm{Cl}(1 \mathrm{D}) & 94.30(18) \\ & & \mathrm{C}(1 \mathrm{D})-\mathrm{Ru}(4)-\mathrm{Cl}(1 \mathrm{D}) & 89.76(16) \\ \mathrm{C}(16 \mathrm{C})-\mathrm{Ru}(3)-\mathrm{C}(1 \mathrm{C}) & 95.0(3) & \mathrm{O}(1 \mathrm{D})-\mathrm{Ru}(4)-\mathrm{Cl}(1 \mathrm{D}) & 88.21(11) \\ \mathrm{C}(16 \mathrm{C})-\mathrm{Ru}(3)-\mathrm{O}(1 \mathrm{C}) & 78.8(2) & \mathrm{C}(16 \mathrm{D})-\mathrm{Ru}(4)-\mathrm{Cl}(2 \mathrm{D}) & 95.58(18) \\ \mathrm{C}(1 \mathrm{C})-\mathrm{Ru}(3)-\mathrm{O}(1 \mathrm{C}) & 173.56(19) & \mathrm{C}(1 \mathrm{D})-\mathrm{Ru}(4)-\mathrm{Cl}(2 \mathrm{D}) & 98.97(16) \\ \mathrm{C}(16 \mathrm{C})-\mathrm{Ru}(3)-\mathrm{Cl}(2 \mathrm{C}) & 95.92(18) & \mathrm{O}(1 \mathrm{D})-\mathrm{Ru}(4)-\mathrm{Cl}(2 \mathrm{D}) & 83.83(11) \\ \mathrm{C}(1 \mathrm{C})-\mathrm{Ru}(3)-\mathrm{Cl}(2 \mathrm{C}) & 92.96(17) & \mathrm{Cl}(1 \mathrm{D})-\mathrm{Ru}(4)-\mathrm{Cl}(2 \mathrm{D}) & 165.77(6) \\ \mathrm{O}(1 \mathrm{C})-\mathrm{Ru}(3)-\mathrm{Cl}(2 \mathrm{C}) & 86.08(12) & \mathrm{C}(16 \mathrm{D})-\mathrm{Ru}(4)-\mathrm{Cl}(4 \mathrm{D}) & 170.76(18) \\ \mathrm{C}(16 \mathrm{C})-\mathrm{Ru}(3)-\mathrm{Cl}(1 \mathrm{C}) & 99.48(18) & \mathrm{C}(1 \mathrm{D})-\mathrm{Ru}(4)-\mathrm{Cl}(4 \mathrm{D}) & 78.66(18) \\ \mathrm{C}(1 \mathrm{C})-\mathrm{Ru}(3)-\mathrm{Cl}(1 \mathrm{C}) & 99.32(17) & \mathrm{O}(1 \mathrm{D})-\mathrm{Ru}(4)-\mathrm{Cl}(4 \mathrm{D}) & 105.56(11) \\ \mathrm{O}(1 \mathrm{C})-\mathrm{Ru}(3)-\mathrm{Cl}(1 \mathrm{C}) & 83.48(11) & \mathrm{Cl}(1 \mathrm{D})-\mathrm{Ru}(4)-\mathrm{Cl}(4 \mathrm{D}) & 94.25(5) \\ \mathrm{Cl}(2 \mathrm{C})-\mathrm{Ru}(3)-\mathrm{Cl}(1 \mathrm{C}) & 159.31(6) & \mathrm{Cl}(2 \mathrm{D})-\mathrm{Ru}(4)-\mathrm{Cl}(4 \mathrm{D}) & 76.66(5)\end{array}$

** Non-bonded distance 
Table 4. Bond lengths $[\AA \AA]$ and angles $\left[^{\circ}\right]$ for CCDC 278852.

\begin{tabular}{|c|c|c|c|}
\hline $\mathrm{Ru}(1)-\mathrm{C}(16 \mathrm{~A})$ & $1.827(6)$ & $C(12 A)-C(13 A)$ & $1.400(10)$ \\
\hline $\mathrm{Ru}(1)-\mathrm{C}(1 \mathrm{~A})$ & $1.972(6)$ & $C(13 A)-C(14 A)$ & $1.365(10)$ \\
\hline $\mathrm{Ru}(1)-\mathrm{O}(1 \mathrm{~A})$ & $2.297(4)$ & $C(14 A)-C(15 A)$ & $1.384(9)$ \\
\hline $\mathrm{Ru}(1)-\mathrm{Cl}(1 \mathrm{~A})$ & $2.3434(16)$ & $C(16 A)-C(17 A)$ & $1.430(8)$ \\
\hline $\mathrm{Ru}(1)-\mathrm{Cl}(2 \mathrm{~A})$ & $2.3506(16)$ & $\mathrm{C}(17 \mathrm{~A})-\mathrm{C}(22 \mathrm{~A})$ & $1.396(8)$ \\
\hline $\mathrm{Cl}(3 \mathrm{~A})-\mathrm{C}(5 \mathrm{~A})$ & $1.718(6)$ & $C(17 \mathrm{~A})-\mathrm{C}(18 \mathrm{~A})$ & $1.387(8)$ \\
\hline $\mathrm{Cl}(4 \mathrm{~A})-\mathrm{C}(9 \mathrm{~A})$ & $1.760(6)$ & $\mathrm{C}(18 \mathrm{~A})-\mathrm{C}(19 \mathrm{~A})$ & $1.385(9)$ \\
\hline $\mathrm{Cl}(5 \mathrm{~A})-\mathrm{C}(11 \mathrm{~A})$ & $1.739(6)$ & $C(19 A)-C(20 A)$ & $1.394(9)$ \\
\hline $\mathrm{Cl}(6 \mathrm{~A})-\mathrm{C}(15 \mathrm{~A})$ & $1.731(7)$ & $C(20 A)-C(21 A)$ & $1.392(9)$ \\
\hline $\mathrm{O}(1 \mathrm{~A})-\mathrm{C}(22 \mathrm{~A})$ & $1.381(7)$ & $C(21 \mathrm{~A})-\mathrm{C}(22 \mathrm{~A})$ & $1.405(8)$ \\
\hline $\mathrm{O}(1 \mathrm{~A})-\mathrm{C}(23 \mathrm{~A})$ & $1.484(7)$ & $C(23 A)-C(25 A)$ & $1.519(8)$ \\
\hline $\mathrm{N}(1 \mathrm{~A})-\mathrm{C}(1 \mathrm{~A})$ & $1.360(8)$ & $C(23 \mathrm{~A})-\mathrm{C}(24 \mathrm{~A})$ & $1.521(8)$ \\
\hline $\mathrm{N}(1 \mathrm{~A})-\mathrm{C}(4 \mathrm{~A})$ & $1.437(8)$ & $\mathrm{Ru}(2)-\mathrm{C}(16 \mathrm{~B})$ & $1.829(6)$ \\
\hline $\mathrm{N}(1 \mathrm{~A})-\mathrm{C}(2 \mathrm{~A})$ & $1.485(7)$ & $\mathrm{Ru}(2)-\mathrm{C}(1 \mathrm{~B})$ & $1.962(6)$ \\
\hline $\mathrm{N}(2 \mathrm{~A})-\mathrm{C}(1 \mathrm{~A})$ & $1.355(7)$ & $\mathrm{Ru}(2)-\mathrm{O}(1 \mathrm{~B})$ & $2.291(4)$ \\
\hline $\mathrm{N}(2 \mathrm{~A})-\mathrm{C}(10 \mathrm{~A})$ & $1.418(8)$ & $\mathrm{Ru}(2)-\mathrm{Cl}(2 \mathrm{~B})$ & $2.3663(16)$ \\
\hline $\mathrm{N}(2 \mathrm{~A})-\mathrm{C}(3 \mathrm{~A})$ & $1.464(7)$ & $\mathrm{Ru}(2)-\mathrm{Cl}(1 \mathrm{~B})$ & $2.3867(16)$ \\
\hline $\mathrm{C}(2 \mathrm{~A})-\mathrm{C}(3 \mathrm{~A})$ & $1.521(8)$ & $\mathrm{Ru}(2)-\mathrm{Cl}(3 \mathrm{~B})$ & $2.7406(16)$ \\
\hline $\mathrm{C}(4 \mathrm{~A})-\mathrm{C}(9 \mathrm{~A})$ & $1.398(8)$ & $\mathrm{Cl}(3 \mathrm{~B})-\mathrm{C}(5 \mathrm{~B})$ & $1.737(6)$ \\
\hline$C(4 A)-C(5 A)$ & $1.409(9)$ & $\mathrm{Cl}(4 \mathrm{~B})-\mathrm{C}(9 \mathrm{~B})$ & $1.763(6)$ \\
\hline $\mathrm{C}(5 \mathrm{~A})-\mathrm{C}(6 \mathrm{~A})$ & $1.374(9)$ & $\mathrm{Cl}(5 \mathrm{~B})-\mathrm{C}(11 \mathrm{~B})$ & $1.741(7)$ \\
\hline$C(6 A)-C(7 A)$ & $1.381(9)$ & $\mathrm{Cl}(6 \mathrm{~B})-\mathrm{C}(15 \mathrm{~B})$ & $1.745(7)$ \\
\hline $\mathrm{C}(7 \mathrm{~A})-\mathrm{C}(8 \mathrm{~A})$ & $1.392(9)$ & $\mathrm{O}(1 \mathrm{~B})-\mathrm{C}(22 \mathrm{~B})$ & $1.397(7)$ \\
\hline$C(8 A)-C(9 A)$ & $1.360(8)$ & $\mathrm{O}(1 \mathrm{~B})-\mathrm{C}(23 \mathrm{~B})$ & $1.467(6)$ \\
\hline$C(10 A)-C(15 A)$ & $1.403(8)$ & $\mathrm{N}(1 \mathrm{~B})-\mathrm{C}(1 \mathrm{~B})$ & $1.381(8)$ \\
\hline$C(10 A)-C(11 A)$ & $1.403(8)$ & N(1B)-C(4B) & $1.411(8)$ \\
\hline$C(11 A)-C(12 A)$ & $1.375(9)$ & $\mathrm{N}(1 \mathrm{~B})-\mathrm{C}(2 \mathrm{~B})$ & $1.483(7)$ \\
\hline
\end{tabular}




\begin{tabular}{|c|c|c|c|}
\hline $\mathrm{N}(2 \mathrm{~B})-\mathrm{C}(1 \mathrm{~B})$ & $1.365(7)$ & $\mathrm{Ru}(3)-\mathrm{Cl}(1 \mathrm{C})$ & $2.3607(15)$ \\
\hline $\mathrm{N}(2 \mathrm{~B})-\mathrm{C}(10 \mathrm{~B})$ & $1.423(8)$ & $\mathrm{Cl}(3 \mathrm{C})-\mathrm{C}(5 \mathrm{C})$ & $1.733(6)$ \\
\hline $\mathrm{N}(2 \mathrm{~B})-\mathrm{C}(3 \mathrm{~B})$ & $1.490(7)$ & $\mathrm{Cl}(4 \mathrm{C})-\mathrm{C}(9 \mathrm{C})$ & $1.746(6)$ \\
\hline$C(2 B)-C(3 B)$ & $1.513(9)$ & $\mathrm{Cl}(5 \mathrm{C})-\mathrm{C}(11 \mathrm{C})$ & $1.737(7)$ \\
\hline $\mathrm{C}(4 \mathrm{~B})-\mathrm{C}(5 \mathrm{~B})$ & $1.382(8)$ & $\mathrm{Cl}(6 \mathrm{C})-\mathrm{C}(15 \mathrm{C})$ & $1.738(6)$ \\
\hline $\mathrm{C}(4 \mathrm{~B})-\mathrm{C}(9 \mathrm{~B})$ & $1.398(9)$ & $\mathrm{O}(1 \mathrm{C})-\mathrm{C}(22 \mathrm{C})$ & $1.391(7)$ \\
\hline $\mathrm{C}(5 \mathrm{~B})-\mathrm{C}(6 \mathrm{~B})$ & $1.386(8)$ & $\mathrm{O}(1 \mathrm{C})-\mathrm{C}(23 \mathrm{C})$ & $1.476(6)$ \\
\hline $\mathrm{C}(6 \mathrm{~B})-\mathrm{C}(7 \mathrm{~B})$ & $1.383(10)$ & $\mathrm{N}(1 \mathrm{C})-\mathrm{C}(1 \mathrm{C})$ & $1.363(7)$ \\
\hline $\mathrm{C}(7 \mathrm{~B})-\mathrm{C}(8 \mathrm{~B})$ & $1.388(10)$ & $\mathrm{N}(1 \mathrm{C})-\mathrm{C}(4 \mathrm{C})$ & $1.413(8)$ \\
\hline $\mathrm{C}(8 \mathrm{~B})-\mathrm{C}(9 \mathrm{~B})$ & $1.394(9)$ & $\mathrm{N}(1 \mathrm{C})-\mathrm{C}(2 \mathrm{C})$ & $1.479(7)$ \\
\hline$C(10 B)-C(11 B)$ & $1.376(9)$ & $\mathrm{N}(2 \mathrm{C})-\mathrm{C}(1 \mathrm{C})$ & $1.386(7)$ \\
\hline$C(10 B)-C(15 B)$ & $1.393(9)$ & $\mathrm{N}(2 \mathrm{C})-\mathrm{C}(10 \mathrm{C})$ & $1.422(7)$ \\
\hline$C(11 B)-C(12 B)$ & $1.383(9)$ & $\mathrm{N}(2 \mathrm{C})-\mathrm{C}(3 \mathrm{C})$ & $1.478(7)$ \\
\hline$C(12 B)-C(13 B)$ & $1.399(10)$ & $C(2 C)-C(3 C)$ & $1.500(9)$ \\
\hline$C(13 B)-C(14 B)$ & $1.384(9)$ & $C(4 C)-C(5 C)$ & $1.391(8)$ \\
\hline$C(14 B)-C(15 B)$ & $1.377(9)$ & $C(4 C)-C(9 C)$ & $1.410(8)$ \\
\hline$C(16 B)-C(17 B)$ & $1.453(8)$ & $C(5 C)-C(6 C)$ & $1.381(8)$ \\
\hline$C(17 B)-C(22 B)$ & $1.395(8)$ & $C(6 C)-C(7 C)$ & $1.371(9)$ \\
\hline $\mathrm{C}(17 \mathrm{~B})-\mathrm{C}(18 \mathrm{~B})$ & $1.402(8)$ & $C(7 C)-C(8 C)$ & $1.408(9)$ \\
\hline$C(18 B)-C(19 B)$ & $1.380(8)$ & $C(8 C)-C(9 C)$ & $1.386(9)$ \\
\hline$C(19 B)-C(20 B)$ & $1.391(9)$ & $C(10 C)-C(11 C)$ & $1.397(8)$ \\
\hline$C(20 B)-C(21 B)$ & $1.400(9)$ & $C(10 C)-C(15 C)$ & $1.403(9)$ \\
\hline $\mathrm{C}(21 \mathrm{~B})-\mathrm{C}(22 \mathrm{~B})$ & $1.382(8)$ & $C(11 C)-C(12 C)$ & $1.370(9)$ \\
\hline$C(23 B)-C(25 B)$ & $1.492(8)$ & $C(12 C)-C(13 C)$ & $1.378(9)$ \\
\hline$C(23 B)-C(24 B)$ & $1.514(8)$ & $C(13 C)-C(14 C)$ & $1.386(9)$ \\
\hline $\mathrm{Ru}(3)-\mathrm{C}(16 \mathrm{C})$ & $1.834(6)$ & $C(14 C)-C(15 C)$ & $1.381(9)$ \\
\hline $\mathrm{Ru}(3)-\mathrm{C}(1 \mathrm{C})$ & $1.956(6)$ & $C(16 C)-C(17 C)$ & $1.451(8)$ \\
\hline $\mathrm{Ru}(3)-\mathrm{O}(1 \mathrm{C})$ & $2.290(4)$ & $C(17 C)-C(22 C)$ & $1.390(8)$ \\
\hline $\mathrm{Ru}(3)-\mathrm{Cl}(2 \mathrm{C})$ & $2.3394(15)$ & $\mathrm{C}(17 \mathrm{C})-\mathrm{C}(18 \mathrm{C})$ & $1.412(8)$ \\
\hline
\end{tabular}




\begin{tabular}{|c|c|c|c|}
\hline C(18C)-C(19C) & $1.378(8)$ & $C(7 D)-C(8 D)$ & $1.364(9)$ \\
\hline$C(19 C)-C(20 C)$ & $1.383(9)$ & $\mathrm{C}(8 \mathrm{D})-\mathrm{C}(9 \mathrm{D})$ & $1.393(8)$ \\
\hline$C(20 C)-C(21 C)$ & $1.398(9)$ & $C(10 D)-C(15 D)$ & $1.394(8)$ \\
\hline$C(21 C)-C(22 C)$ & $1.385(8)$ & $C(10 D)-C(11 D)$ & $1.403(8)$ \\
\hline$C(23 C)-C(24 C)$ & $1.491(8)$ & $C(11 D)-C(12 D)$ & $1.385(8)$ \\
\hline$C(23 C)-C(25 C)$ & $1.509(8)$ & $C(12 D)-C(13 D)$ & $1.389(9)$ \\
\hline $\mathrm{Ru}(4)-\mathrm{C}(16 \mathrm{D})$ & $1.825(6)$ & $C(13 D)-C(14 D)$ & $1.369(9)$ \\
\hline Ru(4)-C(1D) & $1.975(5)$ & $C(14 D)-C(15 D)$ & $1.401(9)$ \\
\hline $\mathrm{Ru}(4)-\mathrm{O}(1 \mathrm{D})$ & $2.300(4)$ & $C(16 D)-C(17 D)$ & $1.437(8)$ \\
\hline $\mathrm{Ru}(4)-\mathrm{Cl}(1 \mathrm{D})$ & $2.3566(15)$ & $C(17 D)-C(22 D)$ & $1.396(8)$ \\
\hline $\mathrm{Ru}(4)-\mathrm{Cl}(2 \mathrm{D})$ & $2.3741(15)$ & $C(17 D)-C(18 D)$ & $1.385(8)$ \\
\hline $\mathrm{Ru}(4)-\mathrm{Cl}(4 \mathrm{D})$ & $2.7614(17)$ & $C(18 D)-C(19 D)$ & $1.389(9)$ \\
\hline $\mathrm{Cl}(3 \mathrm{D})-\mathrm{C}(5 \mathrm{D})$ & $1.730(6)$ & $C(19 D)-C(20 D)$ & $1.377(9)$ \\
\hline $\mathrm{Cl}(4 \mathrm{D})-\mathrm{C}(9 \mathrm{D})$ & $1.738(6)$ & $C(20 D)-C(21 D)$ & $1.398(9)$ \\
\hline $\mathrm{Cl}(5 \mathrm{D})-\mathrm{C}(11 \mathrm{D})$ & $1.737(6)$ & $C(21 D)-C(22 D)$ & $1.386(8)$ \\
\hline $\mathrm{Cl}(6 \mathrm{D})-\mathrm{C}(15 \mathrm{D})$ & $1.722(6)$ & $C(23 D)-C(25 D)$ & $1.517(8)$ \\
\hline $\mathrm{O}(1 \mathrm{D})-\mathrm{C}(22 \mathrm{D})$ & $1.380(7)$ & $C(23 D)-C(24 D)$ & $1.505(9)$ \\
\hline $\mathrm{O}(1 \mathrm{D})-\mathrm{C}(23 \mathrm{D})$ & $1.490(7)$ & & \\
\hline N(1D)-C(1D) & $1.367(7)$ & $C(16 A)-R u(1)-C(1 A)$ & $94.3(2)$ \\
\hline $\mathrm{N}(1 \mathrm{D})-\mathrm{C}(4 \mathrm{D})$ & $1.437(7)$ & $C(16 A)-R u(1)-O(1 A)$ & $78.3(2)$ \\
\hline $\mathrm{N}(1 \mathrm{D})-\mathrm{C}(2 \mathrm{D})$ & $1.475(7)$ & $\mathrm{C}(1 \mathrm{~A})-\mathrm{Ru}(1)-\mathrm{O}(1 \mathrm{~A})$ & $172.6(2)$ \\
\hline $\mathrm{N}(2 \mathrm{D})-\mathrm{C}(1 \mathrm{D})$ & $1.354(7)$ & $C(16 A)-R u(1)-C l(1 A)$ & $96.61(19)$ \\
\hline N(2D)-C(10D) & $1.419(7)$ & $\mathrm{C}(1 \mathrm{~A})-\mathrm{Ru}(1)-\mathrm{Cl}(1 \mathrm{~A})$ & $94.20(18)$ \\
\hline $\mathrm{N}(2 \mathrm{D})-\mathrm{C}(3 \mathrm{D})$ & $1.476(7)$ & $\mathrm{O}(1 \mathrm{~A})-\mathrm{Ru}(1)-\mathrm{Cl}(1 \mathrm{~A})$ & $86.08(11)$ \\
\hline $\mathrm{C}(2 \mathrm{D})-\mathrm{C}(3 \mathrm{D})$ & $1.518(8)$ & $\mathrm{C}(16 \mathrm{~A})-\mathrm{Ru}(1)-\mathrm{Cl}(2 \mathrm{~A})$ & $99.59(19)$ \\
\hline$C(4 D)-C(5 D)$ & $1.396(8)$ & $\mathrm{C}(1 \mathrm{~A})-\mathrm{Ru}(1)-\mathrm{Cl}(2 \mathrm{~A})$ & $98.87(18)$ \\
\hline C(4D)-C(9D) & $1.405(8)$ & $\mathrm{O}(1 \mathrm{~A})-\mathrm{Ru}(1)-\mathrm{Cl}(2 \mathrm{~A})$ & $83.19(11)$ \\
\hline$C(5 D)-C(6 D)$ & $1.392(9)$ & $\mathrm{Cl}(1 \mathrm{~A})-\mathrm{Ru}(1)-\mathrm{Cl}(2 \mathrm{~A})$ & $158.30(6)$ \\
\hline$C(6 D)-C(7 D)$ & $1.366(9)$ & $C(22 \mathrm{~A})-\mathrm{O}(1 \mathrm{~A})-\mathrm{C}(23 \mathrm{~A})$ & $119.0(5)$ \\
\hline
\end{tabular}




\begin{tabular}{|c|c|c|c|}
\hline $\mathrm{C}(22 \mathrm{~A})-\mathrm{O}(1 \mathrm{~A})-\mathrm{Ru}(1)$ & $108.5(3)$ & $\mathrm{C}(12 \mathrm{~A})-\mathrm{C}(11 \mathrm{~A})-\mathrm{Cl}(5 \mathrm{~A})$ & $119.5(5)$ \\
\hline $\mathrm{C}(23 \mathrm{~A})-\mathrm{O}(1 \mathrm{~A})-\mathrm{Ru}(1)$ & $132.5(4)$ & $\mathrm{C}(10 \mathrm{~A})-\mathrm{C}(11 \mathrm{~A})-\mathrm{Cl}(5 \mathrm{~A})$ & $118.1(5)$ \\
\hline $\mathrm{C}(1 \mathrm{~A})-\mathrm{N}(1 \mathrm{~A})-\mathrm{C}(4 \mathrm{~A})$ & $127.5(5)$ & $\mathrm{C}(11 \mathrm{~A})-\mathrm{C}(12 \mathrm{~A})-\mathrm{C}(13 \mathrm{~A})$ & $119.2(7)$ \\
\hline $\mathrm{C}(1 \mathrm{~A})-\mathrm{N}(1 \mathrm{~A})-\mathrm{C}(2 \mathrm{~A})$ & $112.4(5)$ & $\mathrm{C}(14 \mathrm{~A})-\mathrm{C}(13 \mathrm{~A})-\mathrm{C}(12 \mathrm{~A})$ & $119.8(7)$ \\
\hline $\mathrm{C}(4 \mathrm{~A})-\mathrm{N}(1 \mathrm{~A})-\mathrm{C}(2 \mathrm{~A})$ & $118.7(5)$ & $C(13 A)-C(14 A)-C(15 A)$ & $120.3(7)$ \\
\hline$C(1 A)-N(2 A)-C(10 A)$ & $124.2(5)$ & $C(14 A)-C(15 A)-C(10 A)$ & $121.8(6)$ \\
\hline$C(1 \mathrm{~A})-\mathrm{N}(2 \mathrm{~A})-\mathrm{C}(3 \mathrm{~A})$ & $114.1(5)$ & $\mathrm{C}(14 \mathrm{~A})-\mathrm{C}(15 \mathrm{~A})-\mathrm{Cl}(6 \mathrm{~A})$ & $120.5(5)$ \\
\hline$C(10 A)-N(2 A)-C(3 A)$ & $119.1(5)$ & $C(10 A)-C(15 A)-C l(6 A)$ & $117.5(5)$ \\
\hline $\mathrm{N}(2 \mathrm{~A})-\mathrm{C}(1 \mathrm{~A})-\mathrm{N}(1 \mathrm{~A})$ & $105.5(5)$ & $C(17 A)-C(16 A)-R u(1)$ & $120.2(4)$ \\
\hline $\mathrm{N}(2 \mathrm{~A})-\mathrm{C}(1 \mathrm{~A})-\mathrm{Ru}(1)$ & $128.0(5)$ & $C(22 A)-C(17 A)-C(18 A)$ & $118.2(6)$ \\
\hline $\mathrm{N}(1 \mathrm{~A})-\mathrm{C}(1 \mathrm{~A})-\mathrm{Ru}(1)$ & $126.4(4)$ & $C(22 A)-C(17 A)-C(16 A)$ & $118.2(6)$ \\
\hline $\mathrm{N}(1 \mathrm{~A})-\mathrm{C}(2 \mathrm{~A})-\mathrm{C}(3 \mathrm{~A})$ & $100.8(5)$ & $C(18 A)-C(17 A)-C(16 A)$ & $123.6(5)$ \\
\hline $\mathrm{N}(2 \mathrm{~A})-\mathrm{C}(3 \mathrm{~A})-\mathrm{C}(2 \mathrm{~A})$ & $101.6(5)$ & $C(17 A)-C(18 A)-C(19 A)$ & $121.6(6)$ \\
\hline$C(9 A)-C(4 A)-C(5 A)$ & $116.3(6)$ & $C(20 A)-C(19 A)-C(18 A)$ & $119.2(6)$ \\
\hline $\mathrm{C}(9 \mathrm{~A})-\mathrm{C}(4 \mathrm{~A})-\mathrm{N}(1 \mathrm{~A})$ & $122.9(6)$ & $C(19 A)-C(20 A)-C(21 A)$ & $121.3(6)$ \\
\hline $\mathrm{C}(5 \mathrm{~A})-\mathrm{C}(4 \mathrm{~A})-\mathrm{N}(1 \mathrm{~A})$ & $120.8(5)$ & $C(20 A)-C(21 A)-C(22 A)$ & $117.9(6)$ \\
\hline$C(6 A)-C(5 A)-C(4 A)$ & $121.1(6)$ & $\mathrm{O}(1 \mathrm{~A})-\mathrm{C}(22 \mathrm{~A})-\mathrm{C}(17 \mathrm{~A})$ & $113.9(5)$ \\
\hline $\mathrm{C}(6 \mathrm{~A})-\mathrm{C}(5 \mathrm{~A})-\mathrm{Cl}(3 \mathrm{~A})$ & $119.0(5)$ & $\mathrm{O}(1 \mathrm{~A})-\mathrm{C}(22 \mathrm{~A})-\mathrm{C}(21 \mathrm{~A})$ & $124.3(6)$ \\
\hline $\mathrm{C}(4 \mathrm{~A})-\mathrm{C}(5 \mathrm{~A})-\mathrm{Cl}(3 \mathrm{~A})$ & $119.8(5)$ & $C(17 A)-C(22 A)-C(21 A)$ & $121.8(6)$ \\
\hline$C(5 A)-C(6 A)-C(7 A)$ & $120.3(7)$ & $\mathrm{O}(1 \mathrm{~A})-\mathrm{C}(23 \mathrm{~A})-\mathrm{C}(25 \mathrm{~A})$ & $106.3(5)$ \\
\hline$C(8 A)-C(7 A)-C(6 A)$ & $120.2(7)$ & $\mathrm{O}(1 \mathrm{~A})-\mathrm{C}(23 \mathrm{~A})-\mathrm{C}(24 \mathrm{~A})$ & $109.5(5)$ \\
\hline $\mathrm{C}(9 \mathrm{~A})-\mathrm{C}(8 \mathrm{~A})-\mathrm{C}(7 \mathrm{~A})$ & $118.7(6)$ & $C(25 A)-C(23 A)-C(24 A)$ & $111.4(5)$ \\
\hline$C(8 A)-C(9 A)-C(4 A)$ & $123.4(6)$ & $\mathrm{C}(16 \mathrm{~B})-\mathrm{Ru}(2)-\mathrm{C}(1 \mathrm{~B})$ & $98.0(3)$ \\
\hline $\mathrm{C}(8 \mathrm{~A})-\mathrm{C}(9 \mathrm{~A})-\mathrm{Cl}(4 \mathrm{~A})$ & $117.8(5)$ & $\mathrm{C}(16 \mathrm{~B})-\mathrm{Ru}(2)-\mathrm{O}(1 \mathrm{~B})$ & $78.7(2)$ \\
\hline$C(4 A)-C(9 A)-C l(4 A)$ & $118.7(5)$ & $\mathrm{C}(1 \mathrm{~B})-\mathrm{Ru}(2)-\mathrm{O}(1 \mathrm{~B})$ & $175.9(2)$ \\
\hline$C(15 A)-C(10 A)-C(11 A)$ & $115.8(6)$ & $\mathrm{C}(16 \mathrm{~B})-\mathrm{Ru}(2)-\mathrm{Cl}(2 \mathrm{~B})$ & $93.43(18)$ \\
\hline$C(15 A)-C(10 A)-N(2 A)$ & $120.7(6)$ & $\mathrm{C}(1 \mathrm{~B})-\mathrm{Ru}(2)-\mathrm{Cl}(2 \mathrm{~B})$ & $89.33(19)$ \\
\hline$C(11 \mathrm{~A})-\mathrm{C}(10 \mathrm{~A})-\mathrm{N}(2 \mathrm{~A})$ & $123.3(6)$ & $\mathrm{O}(1 \mathrm{~B})-\mathrm{Ru}(2)-\mathrm{Cl}(2 \mathrm{~B})$ & $88.51(11)$ \\
\hline$C(12 A)-C(11 A)-C(10 A)$ & $122.4(6)$ & $\mathrm{C}(16 \mathrm{~B})-\mathrm{Ru}(2)-\mathrm{Cl}(1 \mathrm{~B})$ & $95.47(19)$ \\
\hline
\end{tabular}




\begin{tabular}{|c|c|c|c|}
\hline $\mathrm{C}(1 \mathrm{~B})-\mathrm{Ru}(2)-\mathrm{Cl}(1 \mathrm{~B})$ & $98.87(19)$ & $C(7 B)-C(6 B)-C(5 B)$ & $119.1(6)$ \\
\hline $\mathrm{O}(1 \mathrm{~B})-\mathrm{Ru}(2)-\mathrm{Cl}(1 \mathrm{~B})$ & $83.88(11)$ & $\mathrm{C}(8 \mathrm{~B})-\mathrm{C}(7 \mathrm{~B})-\mathrm{C}(6 \mathrm{~B})$ & $119.7(7)$ \\
\hline $\mathrm{Cl}(2 \mathrm{~B})-\mathrm{Ru}(2)-\mathrm{Cl}(1 \mathrm{~B})$ & $166.92(6)$ & $\mathrm{C}(7 \mathrm{~B})-\mathrm{C}(8 \mathrm{~B})-\mathrm{C}(9 \mathrm{~B})$ & $119.0(7)$ \\
\hline $\mathrm{C}(16 \mathrm{~B})-\mathrm{Ru}(2)-\mathrm{Cl}(3 \mathrm{~B})$ & $171.59(18)$ & $\mathrm{C}(8 \mathrm{~B})-\mathrm{C}(9 \mathrm{~B})-\mathrm{C}(4 \mathrm{~B})$ & $123.4(6)$ \\
\hline $\mathrm{C}(1 \mathrm{~B})-\mathrm{Ru}(2)-\mathrm{Cl}(3 \mathrm{~B})$ & $79.05(18)$ & $\mathrm{C}(8 \mathrm{~B})-\mathrm{C}(9 \mathrm{~B})-\mathrm{Cl}(4 \mathrm{~B})$ & $114.7(6)$ \\
\hline $\mathrm{O}(1 \mathrm{~B})-\mathrm{Ru}(2)-\mathrm{Cl}(3 \mathrm{~B})$ & $104.53(11)$ & $\mathrm{C}(4 \mathrm{~B})-\mathrm{C}(9 \mathrm{~B})-\mathrm{Cl}(4 \mathrm{~B})$ & $122.0(5)$ \\
\hline $\mathrm{Cl}(2 \mathrm{~B})-\mathrm{Ru}(2)-\mathrm{Cl}(3 \mathrm{~B})$ & $94.42(5)$ & $C(11 B)-C(10 B)-C(15 B)$ & $118.0(6)$ \\
\hline $\mathrm{Cl}(1 \mathrm{~B})-\mathrm{Ru}(2)-\mathrm{Cl}(3 \mathrm{~B})$ & $77.32(5)$ & $\mathrm{C}(11 \mathrm{~B})-\mathrm{C}(10 \mathrm{~B})-\mathrm{N}(2 \mathrm{~B})$ & $119.3(6)$ \\
\hline $\mathrm{C}(5 \mathrm{~B})-\mathrm{Cl}(3 \mathrm{~B})-\mathrm{Ru}(2)$ & $95.5(2)$ & $\mathrm{C}(15 \mathrm{~B})-\mathrm{C}(10 \mathrm{~B})-\mathrm{N}(2 \mathrm{~B})$ & $122.5(6)$ \\
\hline $\mathrm{C}(22 \mathrm{~B})-\mathrm{O}(1 \mathrm{~B})-\mathrm{C}(23 \mathrm{~B})$ & $119.4(5)$ & $\mathrm{C}(10 \mathrm{~B})-\mathrm{C}(11 \mathrm{~B})-\mathrm{C}(12 \mathrm{~B})$ & $121.4(7)$ \\
\hline $\mathrm{C}(22 \mathrm{~B})-\mathrm{O}(1 \mathrm{~B})-\mathrm{Ru}(2)$ & $107.9(3)$ & $\mathrm{C}(10 \mathrm{~B})-\mathrm{C}(11 \mathrm{~B})-\mathrm{Cl}(5 \mathrm{~B})$ & $119.2(5)$ \\
\hline $\mathrm{C}(23 \mathrm{~B})-\mathrm{O}(1 \mathrm{~B})-\mathrm{Ru}(2)$ & $131.7(4)$ & $\mathrm{C}(12 \mathrm{~B})-\mathrm{C}(11 \mathrm{~B})-\mathrm{Cl}(5 \mathrm{~B})$ & $119.3(5)$ \\
\hline$C(1 B)-N(1 B)-C(4 B)$ & $128.5(5)$ & $C(11 B)-C(12 B)-C(13 B)$ & $119.7(7)$ \\
\hline $\mathrm{C}(1 \mathrm{~B})-\mathrm{N}(1 \mathrm{~B})-\mathrm{C}(2 \mathrm{~B})$ & $111.0(5)$ & $C(12 B)-C(13 B)-C(14 B)$ & $119.0(7)$ \\
\hline $\mathrm{C}(4 \mathrm{~B})-\mathrm{N}(1 \mathrm{~B})-\mathrm{C}(2 \mathrm{~B})$ & $119.2(5)$ & $C(15 B)-C(14 B)-C(13 B)$ & $120.1(7)$ \\
\hline $\mathrm{C}(1 \mathrm{~B})-\mathrm{N}(2 \mathrm{~B})-\mathrm{C}(10 \mathrm{~B})$ & $126.3(5)$ & $C(14 B)-C(15 B)-C(10 B)$ & $121.3(6)$ \\
\hline $\mathrm{C}(1 \mathrm{~B})-\mathrm{N}(2 \mathrm{~B})-\mathrm{C}(3 \mathrm{~B})$ & $112.6(5)$ & $\mathrm{C}(14 \mathrm{~B})-\mathrm{C}(15 \mathrm{~B})-\mathrm{Cl}(6 \mathrm{~B})$ & $119.2(5)$ \\
\hline $\mathrm{C}(10 \mathrm{~B})-\mathrm{N}(2 \mathrm{~B})-\mathrm{C}(3 \mathrm{~B})$ & $118.9(5)$ & $C(10 B)-C(15 B)-C l(6 B)$ & $119.4(5)$ \\
\hline $\mathrm{N}(2 \mathrm{~B})-\mathrm{C}(1 \mathrm{~B})-\mathrm{N}(1 \mathrm{~B})$ & $106.5(5)$ & $C(17 B)-C(16 B)-R u(2)$ & $119.0(4)$ \\
\hline $\mathrm{N}(2 \mathrm{~B})-\mathrm{C}(1 \mathrm{~B})-\mathrm{Ru}(2)$ & $128.7(5)$ & $\mathrm{C}(22 \mathrm{~B})-\mathrm{C}(17 \mathrm{~B})-\mathrm{C}(18 \mathrm{~B})$ & $118.9(6)$ \\
\hline $\mathrm{N}(1 \mathrm{~B})-\mathrm{C}(1 \mathrm{~B})-\mathrm{Ru}(2)$ & $124.8(4)$ & $\mathrm{C}(22 \mathrm{~B})-\mathrm{C}(17 \mathrm{~B})-\mathrm{C}(16 \mathrm{~B})$ & $118.0(6)$ \\
\hline $\mathrm{N}(1 \mathrm{~B})-\mathrm{C}(2 \mathrm{~B})-\mathrm{C}(3 \mathrm{~B})$ & $102.0(5)$ & $\mathrm{C}(18 \mathrm{~B})-\mathrm{C}(17 \mathrm{~B})-\mathrm{C}(16 \mathrm{~B})$ & $123.1(6)$ \\
\hline $\mathrm{N}(2 \mathrm{~B})-\mathrm{C}(3 \mathrm{~B})-\mathrm{C}(2 \mathrm{~B})$ & $101.6(5)$ & $\mathrm{C}(19 \mathrm{~B})-\mathrm{C}(18 \mathrm{~B})-\mathrm{C}(17 \mathrm{~B})$ & $119.6(6)$ \\
\hline$C(5 B)-C(4 B)-C(9 B)$ & $114.8(6)$ & $\mathrm{C}(20 \mathrm{~B})-\mathrm{C}(19 \mathrm{~B})-\mathrm{C}(18 \mathrm{~B})$ & $120.7(6)$ \\
\hline $\mathrm{C}(5 \mathrm{~B})-\mathrm{C}(4 \mathrm{~B})-\mathrm{N}(1 \mathrm{~B})$ & $124.2(6)$ & $C(19 B)-C(20 B)-C(21 B)$ & $120.7(6)$ \\
\hline $\mathrm{C}(9 \mathrm{~B})-\mathrm{C}(4 \mathrm{~B})-\mathrm{N}(1 \mathrm{~B})$ & $121.0(6)$ & $\mathrm{C}(22 \mathrm{~B})-\mathrm{C}(21 \mathrm{~B})-\mathrm{C}(20 \mathrm{~B})$ & $117.9(6)$ \\
\hline$C(4 B)-C(5 B)-C(6 B)$ & $124.1(6)$ & $\mathrm{C}(21 \mathrm{~B})-\mathrm{C}(22 \mathrm{~B})-\mathrm{O}(1 \mathrm{~B})$ & $124.2(5)$ \\
\hline $\mathrm{C}(4 \mathrm{~B})-\mathrm{C}(5 \mathrm{~B})-\mathrm{Cl}(3 \mathrm{~B})$ & $117.8(5)$ & $\mathrm{C}(21 \mathrm{~B})-\mathrm{C}(22 \mathrm{~B})-\mathrm{C}(17 \mathrm{~B})$ & $122.2(6)$ \\
\hline $\mathrm{C}(6 \mathrm{~B})-\mathrm{C}(5 \mathrm{~B})-\mathrm{Cl}(3 \mathrm{~B})$ & $118.0(5)$ & $\mathrm{O}(1 \mathrm{~B})-\mathrm{C}(22 \mathrm{~B})-\mathrm{C}(17 \mathrm{~B})$ & $113.5(5)$ \\
\hline
\end{tabular}




\begin{tabular}{|c|c|c|c|}
\hline $\mathrm{O}(1 \mathrm{~B})-\mathrm{C}(23 \mathrm{~B})-\mathrm{C}(25 \mathrm{~B})$ & $107.5(5)$ & $\mathrm{N}(1 \mathrm{C})-\mathrm{C}(4 \mathrm{C})-\mathrm{C}(9 \mathrm{C})$ & $120.1(5)$ \\
\hline $\mathrm{O}(1 \mathrm{~B})-\mathrm{C}(23 \mathrm{~B})-\mathrm{C}(24 \mathrm{~B})$ & $108.6(5)$ & $C(6 C)-C(5 C)-C(4 C)$ & $123.0(6)$ \\
\hline $\mathrm{C}(25 \mathrm{~B})-\mathrm{C}(23 \mathrm{~B})-\mathrm{C}(24 \mathrm{~B})$ & 113.2(6) & $\mathrm{C}(6 \mathrm{C})-\mathrm{C}(5 \mathrm{C})-\mathrm{Cl}(3 \mathrm{C})$ & $118.8(5)$ \\
\hline $\mathrm{C}(16 \mathrm{C})-\mathrm{Ru}(3)-\mathrm{C}(1 \mathrm{C})$ & $95.0(3)$ & $\mathrm{C}(4 \mathrm{C})-\mathrm{C}(5 \mathrm{C})-\mathrm{Cl}(3 \mathrm{C})$ & $118.1(5)$ \\
\hline $\mathrm{C}(16 \mathrm{C})-\mathrm{Ru}(3)-\mathrm{O}(1 \mathrm{C})$ & $78.8(2)$ & $C(7 C)-C(6 C)-C(5 C)$ & $120.8(6)$ \\
\hline $\mathrm{C}(1 \mathrm{C})-\mathrm{Ru}(3)-\mathrm{O}(1 \mathrm{C})$ & $173.56(19)$ & $C(6 C)-C(7 C)-C(8 C)$ & $118.7(6)$ \\
\hline $\mathrm{C}(16 \mathrm{C})-\mathrm{Ru}(3)-\mathrm{Cl}(2 \mathrm{C})$ & $95.92(18)$ & $\mathrm{C}(9 \mathrm{C})-\mathrm{C}(8 \mathrm{C})-\mathrm{C}(7 \mathrm{C})$ & $119.4(6)$ \\
\hline $\mathrm{C}(1 \mathrm{C})-\mathrm{Ru}(3)-\mathrm{Cl}(2 \mathrm{C})$ & $92.96(17)$ & $\mathrm{C}(8 \mathrm{C})-\mathrm{C}(9 \mathrm{C})-\mathrm{C}(4 \mathrm{C})$ & $122.8(6)$ \\
\hline $\mathrm{O}(1 \mathrm{C})-\mathrm{Ru}(3)-\mathrm{Cl}(2 \mathrm{C})$ & $86.08(12)$ & $\mathrm{C}(8 \mathrm{C})-\mathrm{C}(9 \mathrm{C})-\mathrm{Cl}(4 \mathrm{C})$ & $116.4(5)$ \\
\hline $\mathrm{C}(16 \mathrm{C})-\mathrm{Ru}(3)-\mathrm{Cl}(1 \mathrm{C})$ & $99.48(18)$ & $\mathrm{C}(4 \mathrm{C})-\mathrm{C}(9 \mathrm{C})-\mathrm{Cl}(4 \mathrm{C})$ & $120.8(5)$ \\
\hline $\mathrm{C}(1 \mathrm{C})-\mathrm{Ru}(3)-\mathrm{Cl}(1 \mathrm{C})$ & $99.32(17)$ & $C(11 C)-C(10 C)-C(15 C)$ & $116.4(6)$ \\
\hline $\mathrm{O}(1 \mathrm{C})-\mathrm{Ru}(3)-\mathrm{Cl}(1 \mathrm{C})$ & $83.48(11)$ & $\mathrm{C}(11 \mathrm{C})-\mathrm{C}(10 \mathrm{C})-\mathrm{N}(2 \mathrm{C})$ & $120.6(6)$ \\
\hline $\mathrm{Cl}(2 \mathrm{C})-\mathrm{Ru}(3)-\mathrm{Cl}(1 \mathrm{C})$ & $159.31(6)$ & $\mathrm{C}(15 \mathrm{C})-\mathrm{C}(10 \mathrm{C})-\mathrm{N}(2 \mathrm{C})$ & $122.6(6)$ \\
\hline$C(22 C)-O(1 C)-C(23 C)$ & $118.8(4)$ & $C(12 C)-C(11 C)-C(10 C)$ & $122.5(6)$ \\
\hline $\mathrm{C}(22 \mathrm{C})-\mathrm{O}(1 \mathrm{C})-\mathrm{Ru}(3)$ & 109.2(3) & $\mathrm{C}(12 \mathrm{C})-\mathrm{C}(11 \mathrm{C})-\mathrm{Cl}(5 \mathrm{C})$ & $119.6(5)$ \\
\hline $\mathrm{C}(23 \mathrm{C})-\mathrm{O}(1 \mathrm{C})-\mathrm{Ru}(3)$ & $131.9(3)$ & $\mathrm{C}(10 \mathrm{C})-\mathrm{C}(11 \mathrm{C})-\mathrm{Cl}(5 \mathrm{C})$ & $117.9(5)$ \\
\hline $\mathrm{C}(1 \mathrm{C})-\mathrm{N}(1 \mathrm{C})-\mathrm{C}(4 \mathrm{C})$ & $126.2(5)$ & $C(11 C)-C(12 C)-C(13 C)$ & $118.9(6)$ \\
\hline $\mathrm{C}(1 \mathrm{C})-\mathrm{N}(1 \mathrm{C})-\mathrm{C}(2 \mathrm{C})$ & $112.6(5)$ & $C(14 C)-C(13 C)-C(12 C)$ & $121.0(6)$ \\
\hline $\mathrm{C}(4 \mathrm{C})-\mathrm{N}(1 \mathrm{C})-\mathrm{C}(2 \mathrm{C})$ & $119.6(5)$ & $C(15 C)-C(14 C)-C(13 C)$ & $118.9(6)$ \\
\hline $\mathrm{C}(1 \mathrm{C})-\mathrm{N}(2 \mathrm{C})-\mathrm{C}(10 \mathrm{C})$ & $124.0(5)$ & $C(14 C)-C(15 C)-C(10 C)$ & $121.5(6)$ \\
\hline $\mathrm{C}(1 \mathrm{C})-\mathrm{N}(2 \mathrm{C})-\mathrm{C}(3 \mathrm{C})$ & $112.2(5)$ & $\mathrm{C}(14 \mathrm{C})-\mathrm{C}(15 \mathrm{C})-\mathrm{Cl}(6 \mathrm{C})$ & $119.8(5)$ \\
\hline $\mathrm{C}(10 \mathrm{C})-\mathrm{N}(2 \mathrm{C})-\mathrm{C}(3 \mathrm{C})$ & $119.7(5)$ & $\mathrm{C}(10 \mathrm{C})-\mathrm{C}(15 \mathrm{C})-\mathrm{Cl}(6 \mathrm{C})$ & $118.7(5)$ \\
\hline $\mathrm{N}(1 \mathrm{C})-\mathrm{C}(1 \mathrm{C})-\mathrm{N}(2 \mathrm{C})$ & $105.6(5)$ & $C(17 C)-C(16 C)-R u(3)$ & $118.9(4)$ \\
\hline $\mathrm{N}(1 \mathrm{C})-\mathrm{C}(1 \mathrm{C})-\mathrm{Ru}(3)$ & $127.4(4)$ & $\mathrm{C}(22 \mathrm{C})-\mathrm{C}(17 \mathrm{C})-\mathrm{C}(18 \mathrm{C})$ & $119.0(5)$ \\
\hline $\mathrm{N}(2 \mathrm{C})-\mathrm{C}(1 \mathrm{C})-\mathrm{Ru}(3)$ & $126.9(4)$ & $C(22 C)-C(17 C)-C(16 C)$ & 119.1(6) \\
\hline $\mathrm{N}(1 \mathrm{C})-\mathrm{C}(2 \mathrm{C})-\mathrm{C}(3 \mathrm{C})$ & $101.9(5)$ & $C(18 C)-C(17 C)-C(16 C)$ & $121.9(6)$ \\
\hline $\mathrm{N}(2 \mathrm{C})-\mathrm{C}(3 \mathrm{C})-\mathrm{C}(2 \mathrm{C})$ & $102.8(5)$ & $\mathrm{C}(19 \mathrm{C})-\mathrm{C}(18 \mathrm{C})-\mathrm{C}(17 \mathrm{C})$ & 119.1(6) \\
\hline $\mathrm{C}(5 \mathrm{C})-\mathrm{C}(4 \mathrm{C})-\mathrm{N}(1 \mathrm{C})$ & $124.6(5)$ & $\mathrm{C}(20 \mathrm{C})-\mathrm{C}(19 \mathrm{C})-\mathrm{C}(18 \mathrm{C})$ & $120.7(6)$ \\
\hline$C(5 C)-C(4 C)-C(9 C)$ & $115.3(6)$ & $C(19 C)-C(20 C)-C(21 C)$ & $121.5(6)$ \\
\hline
\end{tabular}




\begin{tabular}{|c|c|c|c|}
\hline$C(22 C)-C(21 C)-C(20 C)$ & $117.4(6)$ & $\mathrm{C}(1 \mathrm{D})-\mathrm{N}(2 \mathrm{D})-\mathrm{C}(10 \mathrm{D})$ & $124.9(5)$ \\
\hline$C(21 C)-C(22 C)-C(17 C)$ & $122.3(6)$ & $\mathrm{C}(1 \mathrm{D})-\mathrm{N}(2 \mathrm{D})-\mathrm{C}(3 \mathrm{D})$ & $113.1(5)$ \\
\hline $\mathrm{C}(21 \mathrm{C})-\mathrm{C}(22 \mathrm{C})-\mathrm{O}(1 \mathrm{C})$ & $124.7(5)$ & $\mathrm{C}(10 \mathrm{D})-\mathrm{N}(2 \mathrm{D})-\mathrm{C}(3 \mathrm{D})$ & $119.3(5)$ \\
\hline $\mathrm{C}(17 \mathrm{C})-\mathrm{C}(22 \mathrm{C})-\mathrm{O}(1 \mathrm{C})$ & $113.0(5)$ & $\mathrm{N}(2 \mathrm{D})-\mathrm{C}(1 \mathrm{D})-\mathrm{N}(1 \mathrm{D})$ & $106.3(5)$ \\
\hline $\mathrm{O}(1 \mathrm{C})-\mathrm{C}(23 \mathrm{C})-\mathrm{C}(24 \mathrm{C})$ & $106.3(4)$ & $\mathrm{N}(2 \mathrm{D})-\mathrm{C}(1 \mathrm{D})-\mathrm{Ru}(4)$ & $128.6(4)$ \\
\hline $\mathrm{O}(1 \mathrm{C})-\mathrm{C}(23 \mathrm{C})-\mathrm{C}(25 \mathrm{C})$ & $108.9(5)$ & $\mathrm{N}(1 \mathrm{D})-\mathrm{C}(1 \mathrm{D})-\mathrm{Ru}(4)$ & $125.1(4)$ \\
\hline$C(24 C)-C(23 C)-C(25 C)$ & $113.2(5)$ & $\mathrm{N}(1 \mathrm{D})-\mathrm{C}(2 \mathrm{D})-\mathrm{C}(3 \mathrm{D})$ & $101.3(5)$ \\
\hline C(16D)-Ru(4)-C(1D) & $97.9(2)$ & $\mathrm{N}(2 \mathrm{D})-\mathrm{C}(3 \mathrm{D})-\mathrm{C}(2 \mathrm{D})$ & $101.2(5)$ \\
\hline$C(16 D)-R u(4)-O(1 D)$ & $78.2(2)$ & $C(5 D)-C(4 D)-C(9 D)$ & $116.2(6)$ \\
\hline$C(1 D)-R u(4)-O(1 D)$ & $175.4(2)$ & $C(5 D)-C(4 D)-N(1 D)$ & $120.9(5)$ \\
\hline $\mathrm{C}(16 \mathrm{D})-\mathrm{Ru}(4)-\mathrm{Cl}(1 \mathrm{D})$ & $94.30(18)$ & C(9D)-C(4D)-N(1D) & $122.9(5)$ \\
\hline $\mathrm{C}(1 \mathrm{D})-\mathrm{Ru}(4)-\mathrm{Cl}(1 \mathrm{D})$ & $89.76(16)$ & $C(6 D)-C(5 D)-C(4 D)$ & $121.5(6)$ \\
\hline $\mathrm{O}(1 \mathrm{D})-\mathrm{Ru}(4)-\mathrm{Cl}(1 \mathrm{D})$ & $88.21(11)$ & $\mathrm{C}(6 \mathrm{D})-\mathrm{C}(5 \mathrm{D})-\mathrm{Cl}(3 \mathrm{D})$ & $117.1(5)$ \\
\hline $\mathrm{C}(16 \mathrm{D})-\mathrm{Ru}(4)-\mathrm{Cl}(2 \mathrm{D})$ & $95.58(18)$ & $\mathrm{C}(4 \mathrm{D})-\mathrm{C}(5 \mathrm{D})-\mathrm{Cl}(3 \mathrm{D})$ & $121.3(5)$ \\
\hline $\mathrm{C}(1 \mathrm{D})-\mathrm{Ru}(4)-\mathrm{Cl}(2 \mathrm{D})$ & $98.97(16)$ & $C(7 D)-C(6 D)-C(5 D)$ & $119.8(6)$ \\
\hline $\mathrm{O}(1 \mathrm{D})-\mathrm{Ru}(4)-\mathrm{Cl}(2 \mathrm{D})$ & $83.83(11)$ & $C(8 D)-C(7 D)-C(6 D)$ & $121.3(6)$ \\
\hline $\mathrm{Cl}(1 \mathrm{D})-\mathrm{Ru}(4)-\mathrm{Cl}(2 \mathrm{D})$ & $165.77(6)$ & $C(7 D)-C(8 D)-C(9 D)$ & $118.8(6)$ \\
\hline $\mathrm{C}(16 \mathrm{D})-\mathrm{Ru}(4)-\mathrm{Cl}(4 \mathrm{D})$ & $170.76(18)$ & $C(8 D)-C(9 D)-C(4 D)$ & $122.3(6)$ \\
\hline $\mathrm{C}(1 \mathrm{D})-\mathrm{Ru}(4)-\mathrm{Cl}(4 \mathrm{D})$ & $78.66(18)$ & $\mathrm{C}(8 \mathrm{D})-\mathrm{C}(9 \mathrm{D})-\mathrm{Cl}(4 \mathrm{D})$ & $118.9(5)$ \\
\hline $\mathrm{O}(1 \mathrm{D})-\mathrm{Ru}(4)-\mathrm{Cl}(4 \mathrm{D})$ & $105.56(11)$ & $\mathrm{C}(4 \mathrm{D})-\mathrm{C}(9 \mathrm{D})-\mathrm{Cl}(4 \mathrm{D})$ & $118.7(5)$ \\
\hline Cl(1D)-Ru(4)-Cl(4D) & $94.25(5)$ & $C(15 D)-C(10 D)-C(11 D)$ & $117.4(6)$ \\
\hline $\mathrm{Cl}(2 \mathrm{D})-\mathrm{Ru}(4)-\mathrm{Cl}(4 \mathrm{D})$ & $76.66(5)$ & $\mathrm{C}(15 \mathrm{D})-\mathrm{C}(10 \mathrm{D})-\mathrm{N}(2 \mathrm{D})$ & $120.9(5)$ \\
\hline C(9D)-Cl(4D)-Ru(4) & $95.5(2)$ & $C(11 D)-C(10 D)-N(2 D)$ & $121.6(5)$ \\
\hline$C(22 D)-O(1 D)-C(23 D)$ & $119.8(5)$ & $C(12 D)-C(11 D)-C(10 D)$ & $121.4(6)$ \\
\hline $\mathrm{C}(22 \mathrm{D})-\mathrm{O}(1 \mathrm{D})-\mathrm{Ru}(4)$ & $108.0(3)$ & $C(12 D)-C(11 D)-C l(5 D)$ & $119.2(5)$ \\
\hline $\mathrm{C}(23 \mathrm{D})-\mathrm{O}(1 \mathrm{D})-\mathrm{Ru}(4)$ & $131.0(3)$ & $C(10 D)-C(11 D)-C l(5 D)$ & $119.4(5)$ \\
\hline C(1D)-N(1D)-C(4D) & $128.2(5)$ & $C(13 D)-C(12 D)-C(11 D)$ & $118.9(6)$ \\
\hline C(1D)-N(1D)-C(2D) & $111.1(5)$ & $C(12 D)-C(13 D)-C(14 D)$ & $121.7(6)$ \\
\hline $\mathrm{C}(4 \mathrm{D})-\mathrm{N}(1 \mathrm{D})-\mathrm{C}(2 \mathrm{D})$ & 119.3(5) & $\mathrm{C}(13 \mathrm{D})-\mathrm{C}(14 \mathrm{D})-\mathrm{C}(15 \mathrm{D})$ & $118.6(6)$ \\
\hline
\end{tabular}




$\begin{array}{llll}\mathrm{C}(14 \mathrm{D})-\mathrm{C}(15 \mathrm{D})-\mathrm{C}(10 \mathrm{D}) & 121.7(6) & \mathrm{C}(21 \mathrm{D})-\mathrm{C}(20 \mathrm{D})-\mathrm{C}(19 \mathrm{D}) & 122.1(6) \\ \mathrm{C}(14 \mathrm{D})-\mathrm{C}(15 \mathrm{D})-\mathrm{Cl}(6 \mathrm{D}) & 120.9(5) & \mathrm{C}(20 \mathrm{D})-\mathrm{C}(21 \mathrm{D})-\mathrm{C}(22 \mathrm{D}) & 117.6(6) \\ \mathrm{C}(10 \mathrm{D})-\mathrm{C}(15 \mathrm{D})-\mathrm{Cl}(6 \mathrm{D}) & 117.2(5) & \mathrm{O}(1 \mathrm{D})-\mathrm{C}(22 \mathrm{D})-\mathrm{C}(17 \mathrm{D}) & 112.5(5) \\ \mathrm{C}(17 \mathrm{D})-\mathrm{C}(16 \mathrm{D})-\mathrm{Ru}(4) & 118.6(4) & \mathrm{O}(1 \mathrm{D})-\mathrm{C}(22 \mathrm{D})-\mathrm{C}(21 \mathrm{D}) & 124.9(5) \\ \mathrm{C}(22 \mathrm{D})-\mathrm{C}(17 \mathrm{D})-\mathrm{C}(18 \mathrm{D}) & 117.1(6) & \mathrm{C}(17 \mathrm{D})-\mathrm{C}(22 \mathrm{D})-\mathrm{C}(21 \mathrm{D}) & 122.5(6) \\ \mathrm{C}(22 \mathrm{D})-\mathrm{C}(17 \mathrm{D})-\mathrm{C}(16 \mathrm{D}) & 119.1(5) & \mathrm{O}(1 \mathrm{D})-\mathrm{C}(23 \mathrm{D})-\mathrm{C}(25 \mathrm{D}) & 109.1(5) \\ \mathrm{C}(18 \mathrm{D})-\mathrm{C}(17 \mathrm{D})-\mathrm{C}(16 \mathrm{D}) & 123.8(5) & \mathrm{O}(1 \mathrm{D})-\mathrm{C}(23 \mathrm{D})-\mathrm{C}(24 \mathrm{D}) & 107.2(5) \\ \mathrm{C}(19 \mathrm{D})-\mathrm{C}(18 \mathrm{D})-\mathrm{C}(17 \mathrm{D}) & 122.6(6) & \mathrm{C}(25 \mathrm{D})-\mathrm{C}(23 \mathrm{D})-\mathrm{C}(24 \mathrm{D}) & 113.5(6) \\ \mathrm{C}(18 \mathrm{D})-\mathrm{C}(19 \mathrm{D})-\mathrm{C}(20 \mathrm{D}) & 118.0(7) & & \end{array}$


Table 5. Anisotropic displacement parameters $\left(\AA^{2} \mathbf{x} 10^{4}\right)$ for CCDC 278852 . The anisotropic displacement factor exponent takes the form: $-2 \pi^{2}\left[h^{2} a^{* 2} U 11+\ldots+2 h k\right.$ a* $b^{*}$ $\left.\mathbf{U}^{12}\right]$

\begin{tabular}{|c|c|c|c|c|c|c|}
\hline & $\mathrm{U}^{11}$ & $\mathrm{U}^{22}$ & $\mathrm{U}^{33}$ & $\mathrm{U}^{23}$ & $\mathrm{U}^{13}$ & $\mathrm{U}^{12}$ \\
\hline $\mathrm{Ru}(1)$ & $74(2)$ & $139(2)$ & $136(3)$ & $24(2)$ & $13(2)$ & $31(2)$ \\
\hline $\mathrm{Cl}(1 \mathrm{~A})$ & $103(7)$ & $201(7)$ & $208(8)$ & $-37(6)$ & $6(6)$ & $-10(5)$ \\
\hline $\mathrm{Cl}(2 \mathrm{~A})$ & $118(7)$ & $130(6)$ & $221(8)$ & $-16(6)$ & $-7(6)$ & $40(5)$ \\
\hline $\mathrm{Cl}(3 \mathrm{~A})$ & $316(9)$ & $220(8)$ & $238(9)$ & $13(7)$ & $19(7)$ & $-88(7)$ \\
\hline $\mathrm{Cl}(4 \mathrm{~A})$ & $410(10)$ & $167(7)$ & $227(9)$ & $15(7)$ & $-8(7)$ & $-22(7)$ \\
\hline $\mathrm{Cl}(5 \mathrm{~A})$ & $169(8)$ & $238(8)$ & $288(9)$ & $114(7)$ & $2(6)$ & $-31(6)$ \\
\hline $\mathrm{Cl}(6 \mathrm{~A})$ & $151(7)$ & $135(7)$ & $320(10)$ & $64(6)$ & $72(6)$ & $4(5)$ \\
\hline $\mathrm{O}(1 \mathrm{~A})$ & $120(20)$ & $160(20)$ & $160(20)$ & $32(17)$ & $7(17)$ & $37(16)$ \\
\hline $\mathrm{N}(1 \mathrm{~A})$ & $150(30)$ & $100(20)$ & $110(30)$ & 74(19) & $20(19)$ & $70(18)$ \\
\hline $\mathrm{N}(2 \mathrm{~A})$ & $130(30)$ & $130(20)$ & $150(30)$ & $20(20)$ & $-20(20)$ & $39(19)$ \\
\hline$C(1 \mathrm{~A})$ & $70(30)$ & $150(30)$ & $130(30)$ & $-10(20)$ & $20(20)$ & $0(20)$ \\
\hline $\mathrm{C}(2 \mathrm{~A})$ & $190(30)$ & $160(30)$ & $220(40)$ & $0(30)$ & $40(30)$ & $120(20)$ \\
\hline$C(3 \mathrm{~A})$ & $170(30)$ & $110(30)$ & $180(30)$ & $-40(20)$ & $-10(20)$ & $70(20)$ \\
\hline$C(4 A)$ & $130(30)$ & $160(30)$ & $200(40)$ & $80(20)$ & $70(20)$ & $70(20)$ \\
\hline$C(5 \mathrm{~A})$ & $150(30)$ & $150(30)$ & $230(40)$ & $-20(30)$ & $60(30)$ & $-40(20)$ \\
\hline$C(6 A)$ & $170(30)$ & $250(30)$ & $200(40)$ & $10(30)$ & $30(30)$ & $0(30)$ \\
\hline$C(7 A)$ & $230(40)$ & $370(40)$ & $150(40)$ & $20(30)$ & $0(30)$ & $70(30)$ \\
\hline $\mathrm{C}(8 \mathrm{~A})$ & $260(40)$ & $190(30)$ & $280(40)$ & $120(30)$ & $90(30)$ & $80(30)$ \\
\hline $\mathrm{C}(9 \mathrm{~A})$ & $170(30)$ & $150(30)$ & $160(30)$ & $0(20)$ & $60(20)$ & $120(20)$ \\
\hline$C(10 A)$ & $90(30)$ & $140(30)$ & $190(30)$ & $-10(20)$ & $30(20)$ & $20(20)$ \\
\hline$C(11 \mathrm{~A})$ & $100(30)$ & $170(30)$ & $180(30)$ & $90(20)$ & $20(20)$ & $10(20)$ \\
\hline $\mathrm{C}(12 \mathrm{~A})$ & $230(30)$ & $330(40)$ & $80(30)$ & $30(30)$ & $50(30)$ & $100(30)$ \\
\hline$C(13 \mathrm{~A})$ & $300(40)$ & $270(40)$ & $190(40)$ & $-50(30)$ & $-60(30)$ & $100(30)$ \\
\hline $\mathrm{C}(14 \mathrm{~A})$ & $200(30)$ & $140(30)$ & $270(40)$ & $-40(30)$ & $-90(30)$ & $0(20)$ \\
\hline
\end{tabular}




\begin{tabular}{|c|c|c|c|c|c|c|}
\hline$C(15 A)$ & $120(30)$ & $150(30)$ & $200(40)$ & $30(20)$ & $20(20)$ & $40(20)$ \\
\hline$C(16 A)$ & $120(30)$ & $130(30)$ & $130(30)$ & $-30(20)$ & $10(20)$ & $60(20)$ \\
\hline$C(17 A)$ & $150(30)$ & $100(30)$ & $140(30)$ & $0(20)$ & $30(20)$ & $30(20)$ \\
\hline$C(18 \mathrm{~A})$ & $150(30)$ & $150(30)$ & $170(30)$ & $-40(20)$ & $30(20)$ & $-30(20)$ \\
\hline$C(19 A)$ & $230(30)$ & $230(30)$ & $150(30)$ & $-10(30)$ & $40(30)$ & $-70(30)$ \\
\hline$C(20 A)$ & $150(30)$ & $230(30)$ & $230(40)$ & $-160(30)$ & $60(30)$ & $-30(20)$ \\
\hline$C(21 A)$ & $150(30)$ & $150(30)$ & $230(40)$ & $0(30)$ & $20(30)$ & $20(20)$ \\
\hline$C(22 A)$ & $90(30)$ & $270(30)$ & $130(30)$ & $-70(30)$ & $50(20)$ & $-40(20)$ \\
\hline$C(23 \mathrm{~A})$ & $100(30)$ & $170(30)$ & $220(40)$ & $70(30)$ & $10(20)$ & $30(20)$ \\
\hline$C(24 A)$ & $150(30)$ & $220(30)$ & $280(40)$ & $20(30)$ & $0(30)$ & $30(20)$ \\
\hline$C(25 \mathrm{~A})$ & $150(30)$ & $240(30)$ & $320(40)$ & $130(30)$ & $80(30)$ & $30(20)$ \\
\hline $\mathrm{Ru}(2)$ & $65(2)$ & $142(2)$ & $137(3)$ & $16(2)$ & $10(2)$ & $27(2)$ \\
\hline $\mathrm{Cl}(1 \mathrm{~B})$ & $116(7)$ & $136(6)$ & 247(9) & $-15(6)$ & $-15(6)$ & $26(5)$ \\
\hline $\mathrm{Cl}(2 \mathrm{~B})$ & $97(7)$ & 194(7) & $178(8)$ & $-18(6)$ & $14(5)$ & $-24(5)$ \\
\hline $\mathrm{Cl}(3 \mathrm{~B})$ & $221(8)$ & $169(7)$ & $209(8)$ & $29(6)$ & $35(6)$ & $-16(6)$ \\
\hline $\mathrm{Cl}(4 \mathrm{~B})$ & $386(10)$ & $213(8)$ & 266(9) & $-9(7)$ & $89(7)$ & $-45(7)$ \\
\hline $\mathrm{Cl}(5 \mathrm{~B})$ & $158(8)$ & $127(7)$ & $414(11)$ & $40(7)$ & $48(7)$ & $-11(5)$ \\
\hline $\mathrm{Cl}(6 \mathrm{~B})$ & $181(8)$ & $162(7)$ & $313(10)$ & $60(7)$ & $12(6)$ & $-28(6)$ \\
\hline $\mathrm{O}(1 \mathrm{~B})$ & $140(20)$ & $150(20)$ & $130(20)$ & $33(17)$ & $29(17)$ & $83(16)$ \\
\hline $\mathrm{N}(1 \mathrm{~B})$ & $80(20)$ & $180(30)$ & $160(30)$ & $60(20)$ & $23(19)$ & $80(18)$ \\
\hline $\mathrm{N}(2 \mathrm{~B})$ & $80(20)$ & $120(20)$ & $180(30)$ & $0(20)$ & $40(20)$ & $51(18)$ \\
\hline $\mathrm{C}(1 \mathrm{~B})$ & $140(30)$ & $150(30)$ & $110(30)$ & $20(20)$ & $40(20)$ & $-20(20)$ \\
\hline $\mathrm{C}(2 \mathrm{~B})$ & $150(30)$ & $160(30)$ & $310(40)$ & $-10(30)$ & $50(30)$ & $70(20)$ \\
\hline $\mathrm{C}(3 \mathrm{~B})$ & $180(30)$ & $110(30)$ & $270(40)$ & $50(30)$ & $30(30)$ & $60(20)$ \\
\hline $\mathrm{C}(4 \mathrm{~B})$ & $70(30)$ & $240(30)$ & $150(30)$ & $20(30)$ & $50(20)$ & $-10(20)$ \\
\hline $\mathrm{C}(5 \mathrm{~B})$ & $110(30)$ & $140(30)$ & $200(30)$ & $50(20)$ & $50(20)$ & $80(20)$ \\
\hline $\mathrm{C}(6 \mathrm{~B})$ & $160(30)$ & $230(30)$ & $210(40)$ & $90(30)$ & $20(20)$ & $100(20)$ \\
\hline $\mathrm{C}(7 \mathrm{~B})$ & $190(40)$ & $460(50)$ & $160(40)$ & $90(30)$ & $0(30)$ & $100(30)$ \\
\hline
\end{tabular}




\begin{tabular}{|c|c|c|c|c|c|c|}
\hline $\mathrm{C}(8 \mathrm{~B})$ & $170(30)$ & $430(40)$ & $180(40)$ & $30(30)$ & $70(30)$ & $-20(30)$ \\
\hline $\mathrm{C}(9 \mathrm{~B})$ & $130(30)$ & $210(30)$ & $250(40)$ & $100(30)$ & $100(30)$ & $-10(20)$ \\
\hline$C(10 B)$ & $140(30)$ & $180(30)$ & $140(30)$ & $-20(20)$ & $-30(20)$ & $70(20)$ \\
\hline$C(11 B)$ & $170(30)$ & $130(30)$ & $220(40)$ & $70(20)$ & $0(30)$ & $50(20)$ \\
\hline$C(12 B)$ & $260(40)$ & $180(30)$ & $260(40)$ & $-70(30)$ & $-80(30)$ & $60(30)$ \\
\hline$C(13 B)$ & $260(40)$ & $250(30)$ & $140(30)$ & $-60(30)$ & $-50(30)$ & $120(30)$ \\
\hline$C(14 B)$ & $230(40)$ & $220(30)$ & $200(40)$ & $50(30)$ & $20(30)$ & $110(30)$ \\
\hline$C(15 B)$ & $90(30)$ & $160(30)$ & $190(30)$ & $40(20)$ & $10(20)$ & $80(20)$ \\
\hline$C(16 B)$ & $50(30)$ & $150(30)$ & $170(30)$ & $-20(20)$ & $-70(20)$ & $20(20)$ \\
\hline$C(17 B)$ & $70(30)$ & $160(30)$ & $150(30)$ & $-40(20)$ & $0(20)$ & $0(20)$ \\
\hline$C(18 B)$ & $150(30)$ & $160(30)$ & $180(30)$ & $10(20)$ & $50(20)$ & $-30(20)$ \\
\hline$C(19 B)$ & $150(30)$ & $270(30)$ & $140(30)$ & $-70(30)$ & $90(20)$ & $-60(20)$ \\
\hline$C(20 B)$ & $220(30)$ & $200(30)$ & $210(40)$ & $-40(30)$ & $90(30)$ & $-20(20)$ \\
\hline$C(21 B)$ & $120(30)$ & $220(30)$ & $150(30)$ & $-50(30)$ & $0(20)$ & $20(20)$ \\
\hline$C(22 B)$ & $60(30)$ & $130(30)$ & 190(30) & $-20(20)$ & $10(20)$ & $0(20)$ \\
\hline$C(23 B)$ & $120(30)$ & $120(30)$ & $220(40)$ & $50(20)$ & $-20(20)$ & $70(20)$ \\
\hline$C(24 B)$ & $230(30)$ & $140(30)$ & $310(40)$ & $30(30)$ & $80(30)$ & $50(20)$ \\
\hline$C(25 B)$ & $170(30)$ & $280(30)$ & $240(40)$ & $90(30)$ & $20(30)$ & $70(30)$ \\
\hline $\mathrm{Ru}(3)$ & $94(2)$ & $72(2)$ & $133(2)$ & $-15(2)$ & $29(2)$ & $0(2)$ \\
\hline $\mathrm{Cl}(1 \mathrm{C})$ & $157(7)$ & $107(6)$ & 211(8) & $-39(6)$ & $-5(6)$ & $-36(5)$ \\
\hline $\mathrm{Cl}(2 \mathrm{C})$ & $96(7)$ & $271(8)$ & $168(8)$ & $-56(6)$ & $5(5)$ & $23(5)$ \\
\hline $\mathrm{Cl}(3 \mathrm{C})$ & $378(10)$ & $155(7)$ & 221(9) & $-11(6)$ & $0(7)$ & $-70(6)$ \\
\hline $\mathrm{Cl}(4 \mathrm{C})$ & $324(9)$ & 174(7) & 256(9) & $-15(7)$ & $19(7)$ & $-62(6)$ \\
\hline $\mathrm{Cl}(5 \mathrm{C})$ & $139(8)$ & 195(8) & $310(10)$ & $51(7)$ & $58(6)$ & $-34(6)$ \\
\hline $\mathrm{Cl}(6 \mathrm{C})$ & 171(7) & $217(7)$ & $354(10)$ & 134(7) & $29(6)$ & $-25(6)$ \\
\hline $\mathrm{O}(1 \mathrm{C})$ & $140(20)$ & 112(19) & $230(30)$ & $70(17)$ & $120(18)$ & $75(15)$ \\
\hline $\mathrm{N}(1 \mathrm{C})$ & $100(20)$ & $180(30)$ & $130(30)$ & $-70(20)$ & $-32(19)$ & $44(18)$ \\
\hline $\mathrm{N}(2 \mathrm{C})$ & $100(20)$ & $70(20)$ & $190(30)$ & $-35(19)$ & $50(20)$ & $19(17)$ \\
\hline
\end{tabular}




\begin{tabular}{|c|c|c|c|c|c|c|}
\hline$C(1 C)$ & $120(30)$ & $80(20)$ & $130(30)$ & $-30(20)$ & $60(20)$ & $-40(19)$ \\
\hline $\mathrm{C}(2 \mathrm{C})$ & $180(30)$ & $150(30)$ & $270(40)$ & $-40(30)$ & $20(30)$ & $30(20)$ \\
\hline$C(3 C)$ & $170(30)$ & $160(30)$ & $200(40)$ & $40(30)$ & $-10(30)$ & $30(20)$ \\
\hline$C(4 C)$ & $90(30)$ & $160(30)$ & $150(30)$ & $-70(20)$ & $30(20)$ & $-20(20)$ \\
\hline$C(5 C)$ & $100(30)$ & $240(30)$ & $130(30)$ & $-20(20)$ & $-30(20)$ & $-40(20)$ \\
\hline$C(6 C)$ & $290(40)$ & $230(30)$ & $180(40)$ & $40(30)$ & $110(30)$ & $-20(30)$ \\
\hline$C(7 C)$ & $250(40)$ & $220(30)$ & $120(30)$ & $70(30)$ & $-10(30)$ & $-40(30)$ \\
\hline$C(8 C)$ & $250(40)$ & $250(30)$ & $170(40)$ & $-110(30)$ & $30(30)$ & $-40(30)$ \\
\hline$C(9 C)$ & $160(30)$ & $180(30)$ & $150(30)$ & $-20(20)$ & $30(20)$ & $50(20)$ \\
\hline$C(10 C)$ & $120(30)$ & $100(30)$ & $100(30)$ & $20(20)$ & $-20(20)$ & $50(20)$ \\
\hline$C(11 C)$ & $120(30)$ & $120(30)$ & $260(40)$ & $20(30)$ & $0(20)$ & $-10(20)$ \\
\hline$C(12 C)$ & $140(30)$ & $170(30)$ & $220(40)$ & $20(30)$ & $-40(20)$ & $30(20)$ \\
\hline$C(13 C)$ & $240(40)$ & $230(30)$ & $200(40)$ & $-40(30)$ & $-80(30)$ & $80(30)$ \\
\hline$C(14 C)$ & $150(30)$ & $290(30)$ & $210(40)$ & $90(30)$ & $0(30)$ & $80(30)$ \\
\hline$C(15 C)$ & $90(30)$ & $160(30)$ & $320(40)$ & $40(30)$ & $30(30)$ & $30(20)$ \\
\hline$C(16 C)$ & $130(30)$ & $140(30)$ & $150(30)$ & $0(20)$ & $-20(20)$ & $-20(20)$ \\
\hline$C(17 C)$ & $90(30)$ & $150(30)$ & $140(30)$ & $-100(20)$ & $40(20)$ & $0(20)$ \\
\hline$C(18 C)$ & $130(30)$ & $170(30)$ & $130(30)$ & $0(20)$ & $30(20)$ & $-40(20)$ \\
\hline$C(19 C)$ & $160(30)$ & $290(30)$ & $170(30)$ & $-30(30)$ & $90(20)$ & $-60(30)$ \\
\hline$C(20 C)$ & $220(30)$ & $170(30)$ & $210(40)$ & $-20(30)$ & $100(30)$ & $0(20)$ \\
\hline$C(21 C)$ & $210(30)$ & $80(30)$ & $270(40)$ & $-50(20)$ & $140(30)$ & $0(20)$ \\
\hline$C(22 C)$ & 190(30) & $80(20)$ & $60(30)$ & $-10(20)$ & $10(20)$ & $0(20)$ \\
\hline$C(23 C)$ & $150(30)$ & $80(20)$ & $210(30)$ & $-10(20)$ & $70(20)$ & $30(20)$ \\
\hline$C(24 C)$ & $150(30)$ & $190(30)$ & $370(40)$ & $120(30)$ & $50(30)$ & $80(20)$ \\
\hline$C(25 C)$ & $110(30)$ & $230(30)$ & $310(40)$ & $30(30)$ & $30(30)$ & $10(20)$ \\
\hline $\mathrm{Ru}(4)$ & $94(2)$ & $80(2)$ & $138(3)$ & $2(2)$ & $22(2)$ & $6(2)$ \\
\hline $\mathrm{Cl}(1 \mathrm{D})$ & $88(6)$ & $220(7)$ & $157(8)$ & $-31(6)$ & $6(5)$ & $17(5)$ \\
\hline $\mathrm{Cl}(2 \mathrm{D})$ & $152(7)$ & $129(6)$ & 213(8) & $-25(6)$ & $-7(6)$ & $-27(5)$ \\
\hline
\end{tabular}




\begin{tabular}{|c|c|c|c|c|c|c|}
\hline $\mathrm{Cl}(3 \mathrm{D})$ & $418(10)$ & $176(8)$ & 261(10) & $-16(7)$ & $32(7)$ & $-14(7)$ \\
\hline $\mathrm{Cl}(4 \mathrm{D})$ & $225(8)$ & $167(7)$ & $216(8)$ & $16(6)$ & $16(6)$ & $-28(6)$ \\
\hline $\mathrm{Cl}(5 \mathrm{D})$ & $170(7)$ & $153(7)$ & $219(8)$ & $45(6)$ & $12(6)$ & $-27(5)$ \\
\hline $\mathrm{Cl}(6 \mathrm{D})$ & $145(8)$ & $207(8)$ & $325(10)$ & $68(7)$ & $46(6)$ & $-49(6)$ \\
\hline $\mathrm{O}(1 \mathrm{D})$ & $160(20)$ & $140(20)$ & $170(20)$ & $58(17)$ & $55(17)$ & $19(16)$ \\
\hline $\mathrm{N}(1 \mathrm{D})$ & $120(20)$ & $150(20)$ & $80(30)$ & $-53(19)$ & $39(19)$ & $36(18)$ \\
\hline $\mathrm{N}(2 \mathrm{D})$ & $140(20)$ & $120(20)$ & $70(20)$ & $-49(19)$ & $2(18)$ & $51(18)$ \\
\hline$C(1 D)$ & $60(30)$ & $80(20)$ & $160(30)$ & $-40(20)$ & $0(20)$ & $-7(18)$ \\
\hline$C(2 D)$ & $90(30)$ & $370(40)$ & $150(30)$ & $20(30)$ & $40(20)$ & $100(30)$ \\
\hline$C(3 D)$ & $130(30)$ & $220(30)$ & $210(40)$ & $-60(30)$ & $-20(20)$ & $70(20)$ \\
\hline$C(4 D)$ & $90(30)$ & $150(30)$ & $110(30)$ & $0(20)$ & $50(20)$ & $0(20)$ \\
\hline$C(5 D)$ & $150(30)$ & $150(30)$ & $210(40)$ & $-60(20)$ & $60(20)$ & $20(20)$ \\
\hline$C(6 D)$ & $190(30)$ & $180(30)$ & $190(40)$ & $-60(30)$ & $60(30)$ & $-30(20)$ \\
\hline$C(7 D)$ & $200(30)$ & $300(40)$ & $150(40)$ & $10(30)$ & $0(30)$ & $10(30)$ \\
\hline$C(8 D)$ & $180(30)$ & $240(30)$ & $230(40)$ & $50(30)$ & $40(30)$ & $0(30)$ \\
\hline$C(9 D)$ & $70(30)$ & $210(30)$ & $160(30)$ & $-30(20)$ & $20(20)$ & $-40(20)$ \\
\hline$C(10 D)$ & $80(30)$ & $80(20)$ & $150(30)$ & $-20(20)$ & $30(20)$ & $-11(19)$ \\
\hline$C(11 D)$ & $110(30)$ & $110(30)$ & $210(30)$ & $20(20)$ & $0(20)$ & $0(20)$ \\
\hline$C(12 D)$ & $190(30)$ & $180(30)$ & $160(30)$ & $30(20)$ & $60(20)$ & $20(20)$ \\
\hline$C(13 D)$ & $280(40)$ & $210(30)$ & $130(30)$ & $-10(30)$ & $-20(30)$ & $50(30)$ \\
\hline$C(14 D)$ & $180(30)$ & $140(30)$ & $340(40)$ & $-10(30)$ & $-50(30)$ & $-40(20)$ \\
\hline$C(15 D)$ & $170(30)$ & $110(30)$ & $150(30)$ & $-10(20)$ & $20(20)$ & $-30(20)$ \\
\hline$C(16 D)$ & $80(30)$ & $120(30)$ & $200(30)$ & $-50(20)$ & $0(20)$ & $20(20)$ \\
\hline$C(17 D)$ & $150(30)$ & $100(30)$ & $110(30)$ & $0(20)$ & $-10(20)$ & $10(20)$ \\
\hline$C(18 D)$ & $110(30)$ & $190(30)$ & $240(40)$ & $-50(30)$ & $20(20)$ & $10(20)$ \\
\hline$C(19 D)$ & $220(40)$ & $290(40)$ & $170(40)$ & $-40(30)$ & $30(30)$ & $-40(30)$ \\
\hline$C(20 D)$ & $200(30)$ & $230(30)$ & $250(40)$ & $-140(30)$ & $110(30)$ & $-20(30)$ \\
\hline$C(21 D)$ & $130(30)$ & $100(30)$ & $240(40)$ & $-30(20)$ & $50(20)$ & $0(20)$ \\
\hline$C(22 D)$ & $110(30)$ & $110(30)$ & $190(30)$ & $-70(20)$ & $60(20)$ & $-20(20)$ \\
\hline
\end{tabular}




\begin{tabular}{lllllll}
$\mathrm{C}(23 \mathrm{D})$ & $110(30)$ & $160(30)$ & $270(40)$ & $60(30)$ & $0(20)$ & $70(20)$ \\
$\mathrm{C}(24 \mathrm{D})$ & $240(30)$ & $170(30)$ & $250(40)$ & $50(30)$ & $0(30)$ & $40(20)$ \\
$\mathrm{C}(25 \mathrm{D})$ & $180(30)$ & $180(30)$ & $240(40)$ & $20(30)$ & $30(30)$ & $30(20)$ \\
\hline
\end{tabular}


Table 6. Torsion angles $\left[^{\circ}\right]$ for CCDC 278852.

\begin{tabular}{|c|c|}
\hline $\mathrm{C}(1 \mathrm{~A})-\mathrm{N}(1 \mathrm{~A})-\mathrm{C}(4 \mathrm{~A})-\mathrm{C}(9 \mathrm{~A})$ & $54.0(9)$ \\
\hline $\mathrm{C}(1 \mathrm{C})-\mathrm{N}(1 \mathrm{C})-\mathrm{C}(4 \mathrm{C})-\mathrm{C}(9 \mathrm{C})$ & $130.3(6)$ \\
\hline $\mathrm{C}(1 \mathrm{~B})-\mathrm{N}(1 \mathrm{~B})-\mathrm{C}(4 \mathrm{~B})-\mathrm{C}(9 \mathrm{~B})$ & $133.5(7)$ \\
\hline $\mathrm{C}(1 \mathrm{D})-\mathrm{N}(1 \mathrm{D})-\mathrm{C}(4 \mathrm{D})-\mathrm{C}(9 \mathrm{D})$ & $47.7(9)$ \\
\hline $\mathrm{Ru}(1)-\mathrm{O}(1 \mathrm{~A})-\mathrm{C}(23 \mathrm{~A})-\mathrm{C}(24 \mathrm{~A})$ & $103.7(5)$ \\
\hline $\mathrm{Ru}(3)-\mathrm{O}(1 \mathrm{C})-\mathrm{C}(23 \mathrm{C})-\mathrm{C}(25 \mathrm{C})$ & $-104.3(5)$ \\
\hline $\mathrm{Ru}(1)-\mathrm{O}(1 \mathrm{~A})-\mathrm{C}(23 \mathrm{~A})-\mathrm{C}(25 \mathrm{~A})$ & $-16.7(7)$ \\
\hline $\mathrm{Ru}(3)-\mathrm{O}(1 \mathrm{C})-\mathrm{C}(23 \mathrm{C})-\mathrm{C}(24 \mathrm{C})$ & $18.1(7)$ \\
\hline $\mathrm{Ru}(2)-\mathrm{O}(1 \mathrm{~B})-\mathrm{C}(23 \mathrm{~B})-\mathrm{C}(24 \mathrm{~B})$ & $78.1(6)$ \\
\hline $\mathrm{Ru}(4)-\mathrm{O}(1 \mathrm{D})-\mathrm{C}(23 \mathrm{D})-\mathrm{C}(25 \mathrm{D})$ & $-79.9(6)$ \\
\hline $\mathrm{Ru}(4)-\mathrm{O}(1 \mathrm{D})-\mathrm{C}(23 \mathrm{D})-\mathrm{C}(24 \mathrm{D})$ & $43.4(6)$ \\
\hline $\mathrm{Ru}(2)-\mathrm{O}(1 \mathrm{~B})-\mathrm{C}(23 \mathrm{~B})-\mathrm{C}(25 \mathrm{~B})$ & $-44.7(7)$ \\
\hline $\mathrm{N}(2 \mathrm{~A})-\mathrm{C}(1 \mathrm{~A})-\mathrm{Ru}(1)-\mathrm{C}(16 \mathrm{~A})$ & $-38.0(6)$ \\
\hline $\mathrm{N}(2 \mathrm{C})-\mathrm{C}(1 \mathrm{C})-\mathrm{Ru}(3)-\mathrm{C}(16 \mathrm{C})$ & $37.6(5)$ \\
\hline $\mathrm{N}(2 \mathrm{~B})-\mathrm{C}(1 \mathrm{~B})-\mathrm{Ru}(2)-\mathrm{C}(16 \mathrm{~B})$ & $36.1(6)$ \\
\hline $\mathrm{N}(2 \mathrm{D})-\mathrm{C}(1 \mathrm{D})-\mathrm{Ru}(4)-\mathrm{C}(16 \mathrm{D})$ & $-33.7(5)$ \\
\hline
\end{tabular}




\section{cis( $\mathrm{F}_{4} \mathrm{NHC}-$ phosphine)-derived ruthenium complex S7}

To ruthenium benzylidene 5 (69 $\mathrm{mg}, 82 \mu \mathrm{mol}, 1.0$ equiv) in toluene $(2 \mathrm{ml})$ was added 1isopropoxy-4-nitro-2-vinyl-benzene ${ }^{3}$ (145 $\mathrm{mg}, 0.82 \mathrm{mmol}, 10.0$ equiv). The dark red solution is left inside a capped vial without stirring. After $16 \mathrm{~h}$ at $23{ }^{\circ} \mathrm{C}$ the vial is placed in a bigger vial containing $2 \mathrm{ml}$ of pentane and the pentane is allowed to diffuse in the smaller vial over $16 \mathrm{~h}$. The liquid is decanted off, leaving behind crystalline S7. The crystals are washed with pentane $(2 \times 0.2 \mathrm{ml})$ and dried to afford crystals $(8 \%$ yield $)$. The complex is stable in air for extended periods of time in the solid state and in solution; it is soluble in $\mathrm{CH}_{2} \mathrm{Cl}_{2}$ and $\mathrm{CHCl}_{3}$, and toluene, but not in diethylether and pentane. Crystals suitable for X-ray crystallography were grown by slow diffusion of pentane into solution of S7 in toluene at $25^{\circ} \mathrm{C}$.

NMR Spectroscopy: ${ }^{1} \mathrm{H}$ NMR (300 MHz, $\left.\mathrm{CD}_{2} \mathrm{Cl}_{2}\right) \delta: 16.66(\mathrm{dd}, J=22.8,1.6 \mathrm{~Hz}, 1 \mathrm{H}), 10.43$ (d, $J=3.0 \mathrm{~Hz}, 1 \mathrm{H}), 8.43(\mathrm{dd}, J=9.0 \mathrm{~Hz}, 3.0 \mathrm{~Hz}, 1 \mathrm{H}), 7.56-6.40(\mathrm{~m}, 7 \mathrm{H}), 4.57(\mathrm{qn}, J=6.6 \mathrm{~Hz}, 1 \mathrm{H})$, $4.31(\mathrm{~s}, 4 \mathrm{H}), 1.52(\mathrm{~d}, J=6.2 \mathrm{~Hz}, 6 \mathrm{H}) .{ }^{31} \mathrm{P} \mathrm{NMR}\left(121 \mathrm{MHz}, \mathrm{CD}_{2} \mathrm{Cl}_{2}\right) \delta: 44.98 .{ }^{19} \mathrm{~F}$ NMR $(282$ $\mathrm{MHz}, \mathrm{CD}_{2} \mathrm{Cl}_{2}$ ) $\delta$ : $-102.8,-112.5,-115.0$. Mass Spectrometry HR-FAB NBA Calcd for $\mathrm{C}_{43} \mathrm{H}_{54} \mathrm{~N}_{3} \mathrm{~F}_{4} \mathrm{ClO}_{3} \mathrm{PRu}$, 904.2571. Found, 904.2549.

${ }^{3}$ Michrowska, A.; Bujok, R.; Harutyunyan,S.; Sashuk, V.; Dolgonos, G.; Grela, K. J. Am. Chem. Soc. 2004, 126, 9318-9325. 


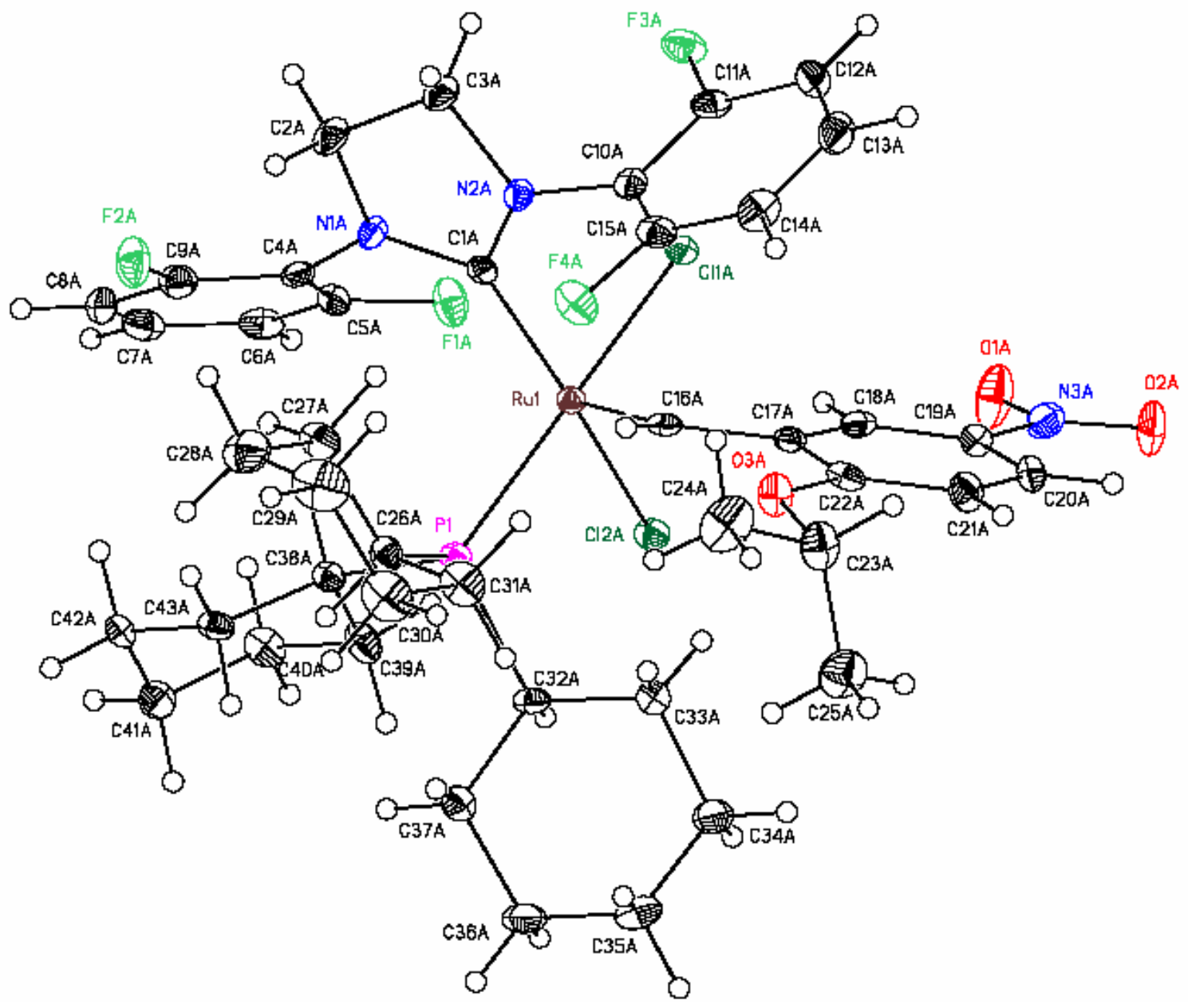




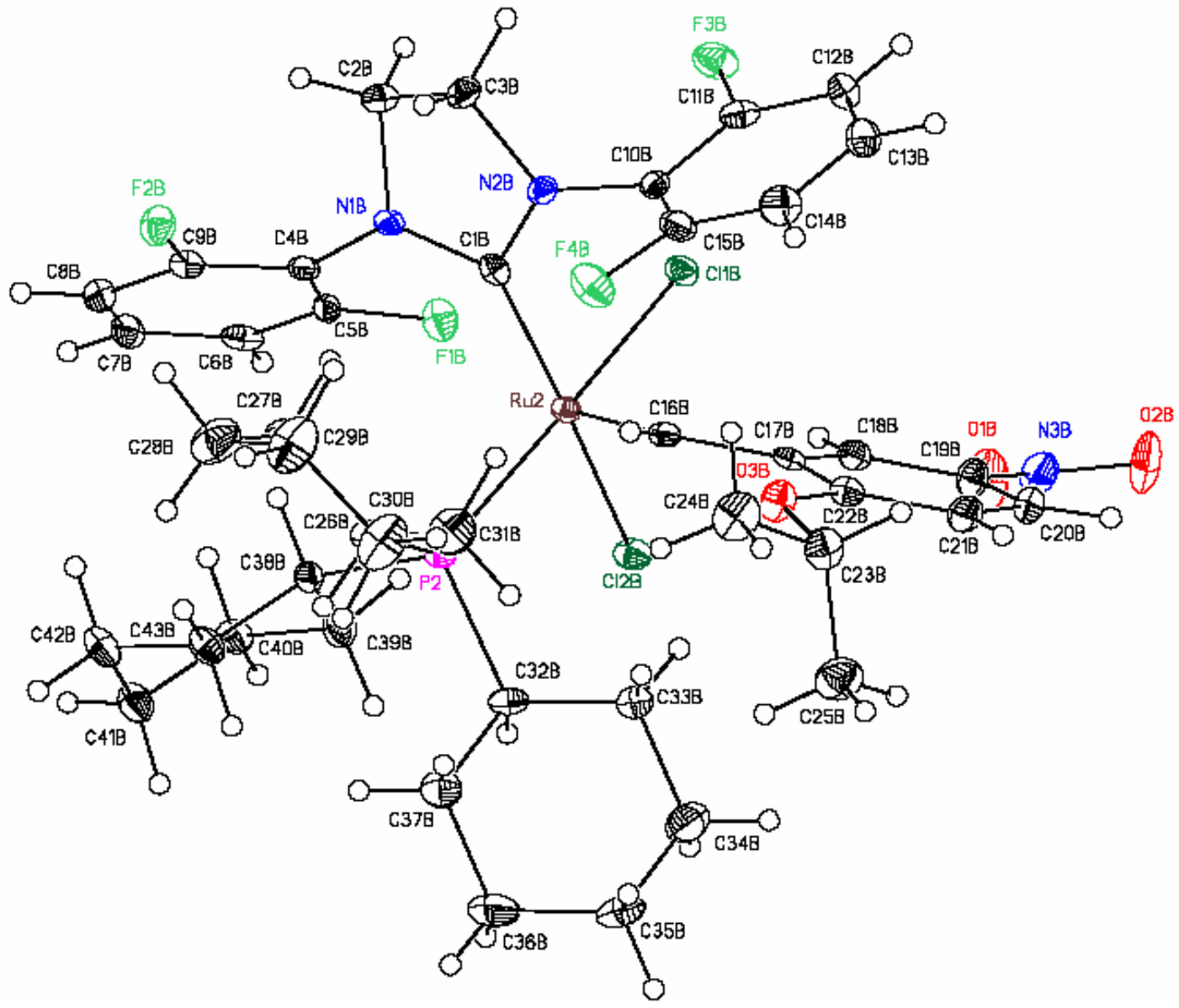


Table 1. Crystal data and structure refinement for CCDC 284775.

$\begin{array}{ll}\text { Empirical formula } & \mathrm{C}_{43} \mathrm{H}_{54} \mathrm{~F}_{4} \mathrm{~N}_{3} \mathrm{O}_{3} \mathrm{PCl}_{2} \mathrm{Ru} \cdot 2\left(\mathrm{CH}_{2} \mathrm{Cl}_{2}\right) \\ \text { Formula weight } & 1109.68 \\ \text { Crystallization Solvent } & \text { Dichloromethane } \\ \text { Crystal Habit } & \text { Needle } \\ \text { Crystal size } & 0.37 \times 0.15 \times 0.15 \mathrm{~mm}^{3} \\ \text { Crystal color } & \text { Green }\end{array}$

\section{Data Collection}

Type of diffractometer

Wavelength

Data Collection Temperature

$\theta$ range for 37599 reflections used

in lattice determination

Unit cell dimensions

Volume

Z

Crystal system

Space group

Density (calculated)

$\mathrm{F}(000)$

Data collection program

$\theta$ range for data collection
Bruker SMART 1000

$0.71073 \AA \mathrm{MoK} \alpha$

100(2) K

2.23 to $31.12^{\circ}$

$a=14.8554(6) \AA$

$\alpha=102.6810(10)^{\circ}$

$\mathrm{b}=17.3381(7) \AA$

$\beta=97.2610(10)^{\circ}$

$\mathrm{c}=19.4448(8) \AA$

$\gamma=93.0900(10)^{\circ}$

4829.7(3) $\AA^{3}$

4

Triclinic

P-1

$1.526 \mathrm{Mg} / \mathrm{m}^{3}$

2280

Bruker SMART v5.630

1.43 to $31.48^{\circ}$ 
Completeness to $\theta=31.48^{\circ}$

Index ranges

Data collection scan type

Data reduction program

Reflections collected

Independent reflections

Absorption coefficient

Absorption correction

Max. and min. transmission
$78.7 \%$

$-21 \leq \mathrm{h} \leq 19,-25 \leq \mathrm{k} \leq 23,-27 \leq 1 \leq 27$

$\omega$ scans at $7 \phi$ settings

Bruker SAINT v6.45A

116793

$25286\left[\mathrm{R}_{\text {int }}=0.0709\right]$

$0.748 \mathrm{~mm}^{-1}$

None

0.8960 and 0.7693 


\section{Table 1 (cont.)}

\section{Structure solution and Refinement}

\begin{tabular}{ll} 
Structure solution program & Bruker XS v6.12 \\
Primary solution method & Direct methods \\
Secondary solution method & Difference Fourier map \\
Hydrogen placement & Geometric positions \\
Structure refinement program & Bruker XL v6.12 \\
Refinement method & Full matrix least-squares on $\mathrm{F}^{2}$ \\
Data / restraints / parameters & $25286 / 0 / 1135$ \\
Treatment of hydrogen atoms & Riding \\
Goodness-of-fit on $\mathrm{F}^{2}$ & 1.562 \\
Final R indices [I $>2 \sigma(\mathrm{I}), 16731$ reflections $]$ & $\mathrm{R} 1=0.0454, w \mathrm{R} 2=0.0724$ \\
R indices (all data) & $\mathrm{R} 1=0.0860, w \mathrm{R} 2=0.0772$ \\
Type of weighting scheme used & Sigma \\
Weighting scheme used & $w=1 / \sigma^{2}\left(\mathrm{Fo}^{2}\right)$ \\
Max shift/error & 0.002 \\
Average shift/error & 0.000 \\
& 2.303 and $-1.604 \mathrm{e} . \AA^{-3}$ \\
\hline
\end{tabular}

\section{Special Refinement Details}

Refinement of $\mathrm{F}^{2}$ against ALL reflections. The weighted R-factor $(w \mathrm{R})$ and goodness of fit (S) are based on $\mathrm{F}^{2}$, conventional R-factors (R) are based on $\mathrm{F}$, with $\mathrm{F}$ set to zero for negative $\mathrm{F}^{2}$. The threshold expression of $\mathrm{F}^{2}$ $>2 \sigma\left(\mathrm{F}^{2}\right)$ is used only for calculating R-factors(gt) etc. and is not relevant to the choice of reflections for refinement. R-factors based on $\mathrm{F}^{2}$ are statistically about twice as large as those based on $\mathrm{F}$, and R-factors based on ALL data will be even larger.

All esds (except the esd in the dihedral angle between two 1.s. planes) are estimated using the full covariance matrix. The cell esds are taken into account individually in the estimation of esds in distances, angles and torsion angles; correlations between esds in cell parameters are only used when they are defined by crystal 
symmetry. An approximate (isotropic) treatment of cell esds is used for estimating esds involving l.s. planes. 


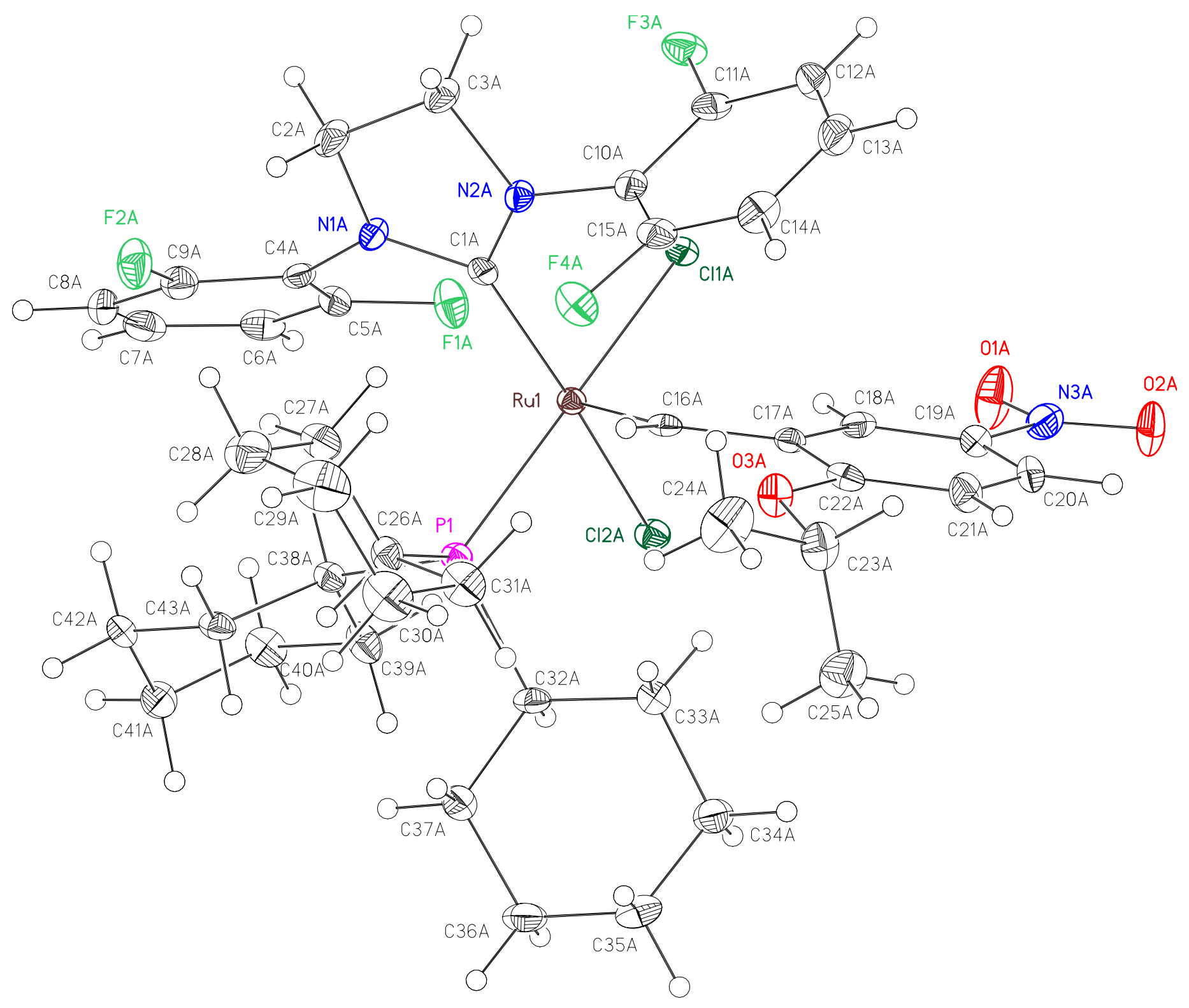

Figure 1. Molecule A. 


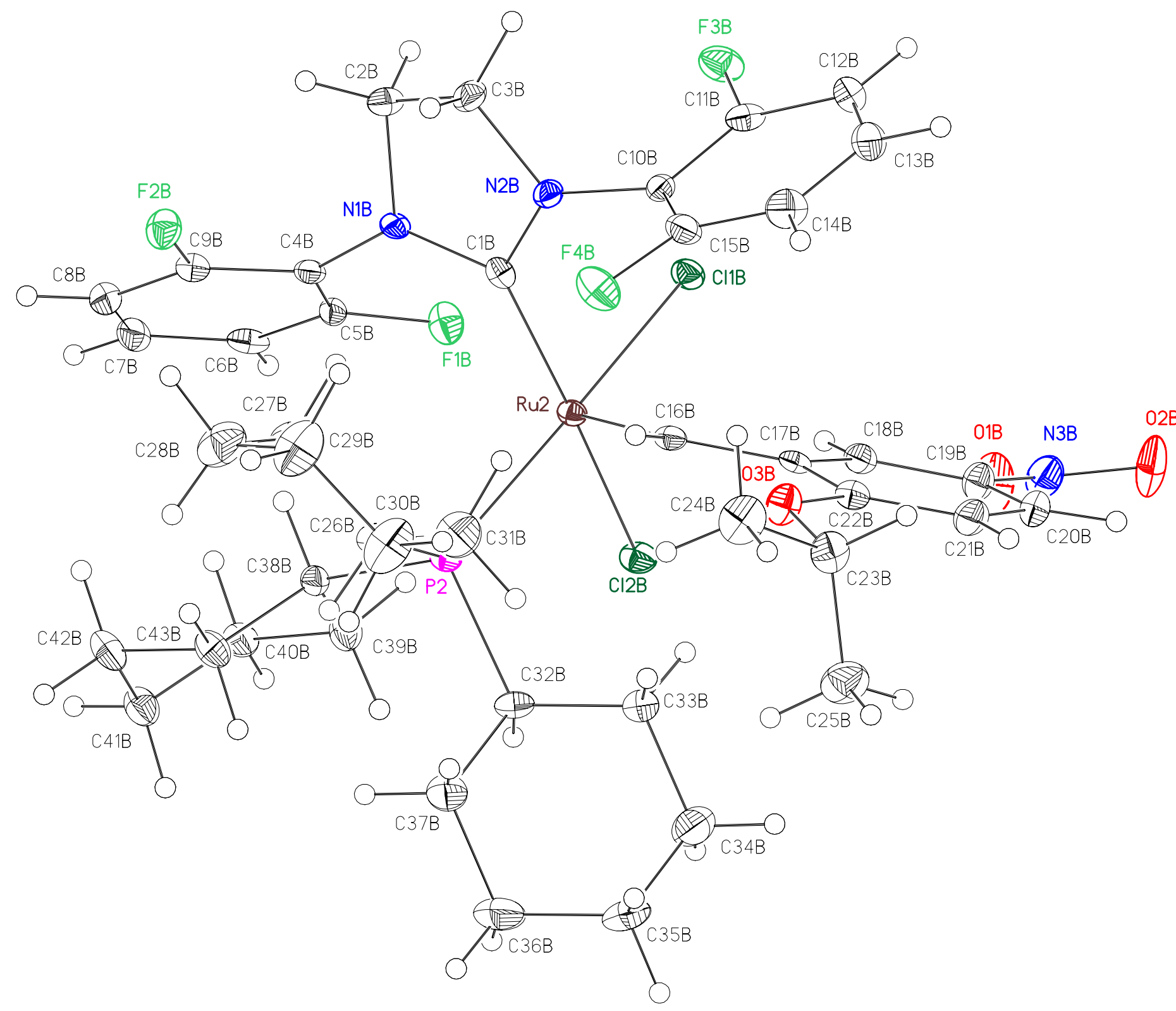

Figure 2. Molecule B. 


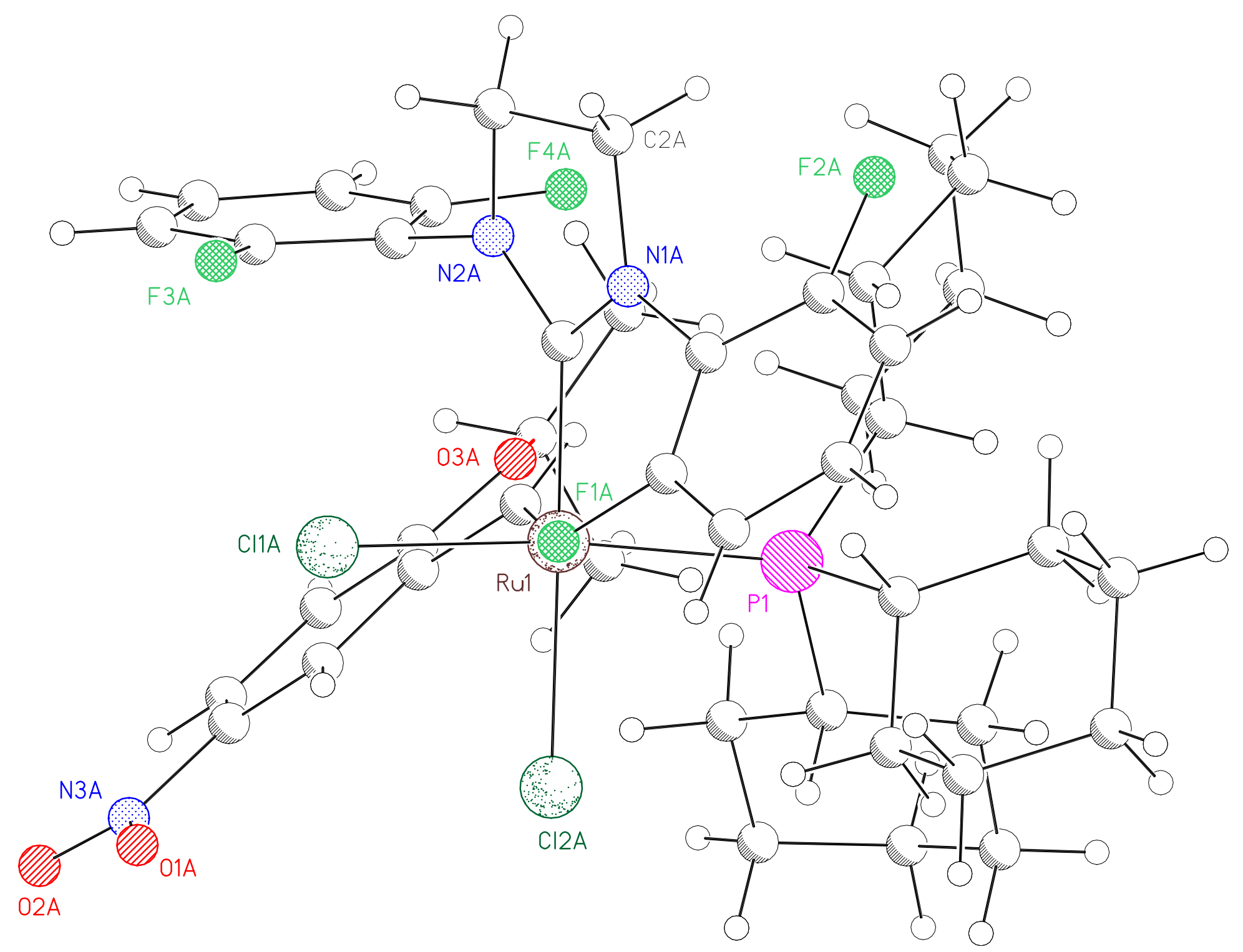

Figure 3. Molecule A viewed down the F1-Ru vector. 


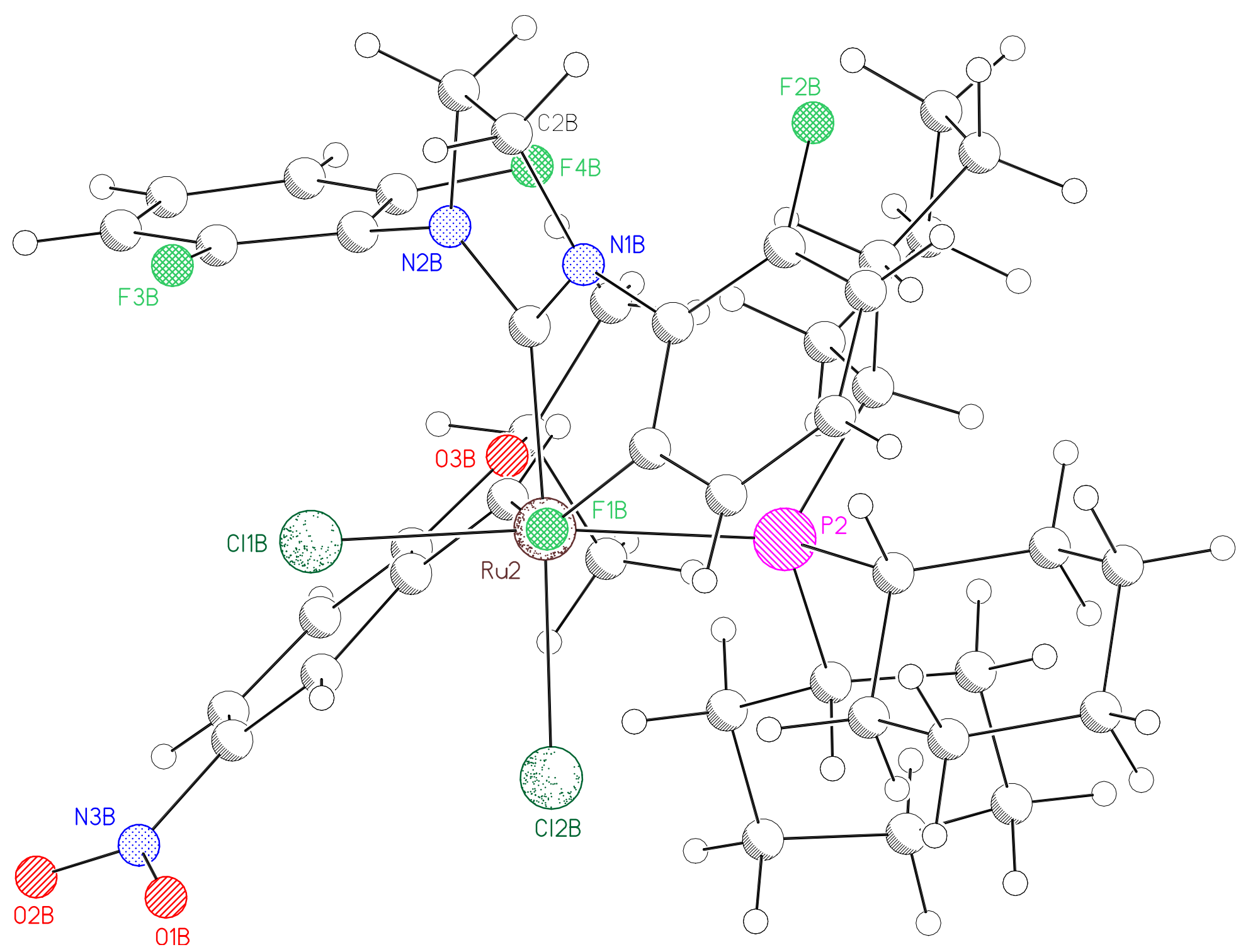

Figure 4. Molecule B viewed down the F1-Ru vector. 


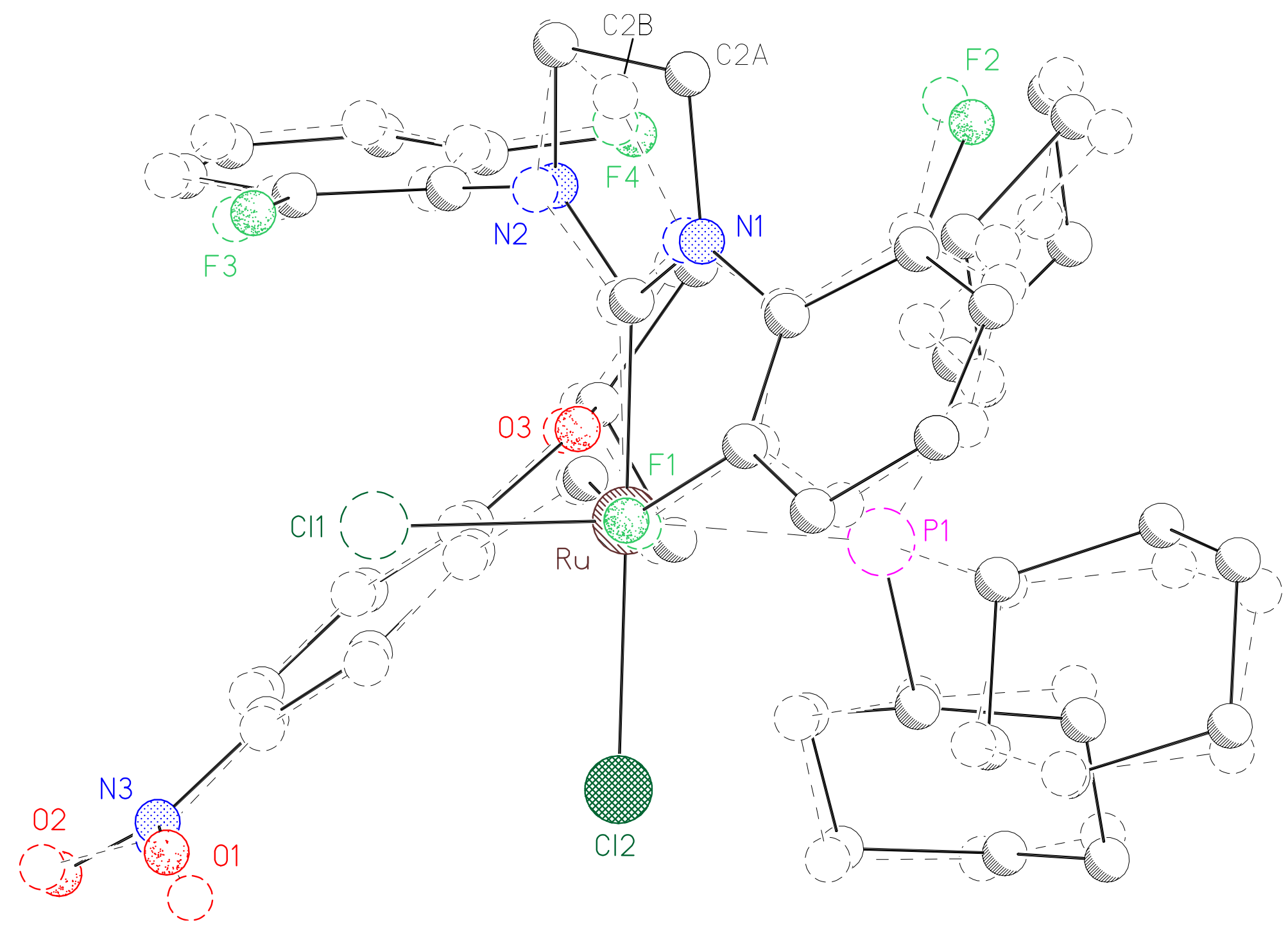

Figure 5. Molecules A and B overlapped and viewed down the F1-Ru vector. The overlap is defined by $\mathrm{Ru}, \mathrm{Cl1}, \mathrm{Cl} 2$ and $\mathrm{P}$. hydrogen atoms are omitted for clarity. The major difference between the two molecules is the pucker in the imidazole ring, primarily at $\mathrm{C} 2$. 


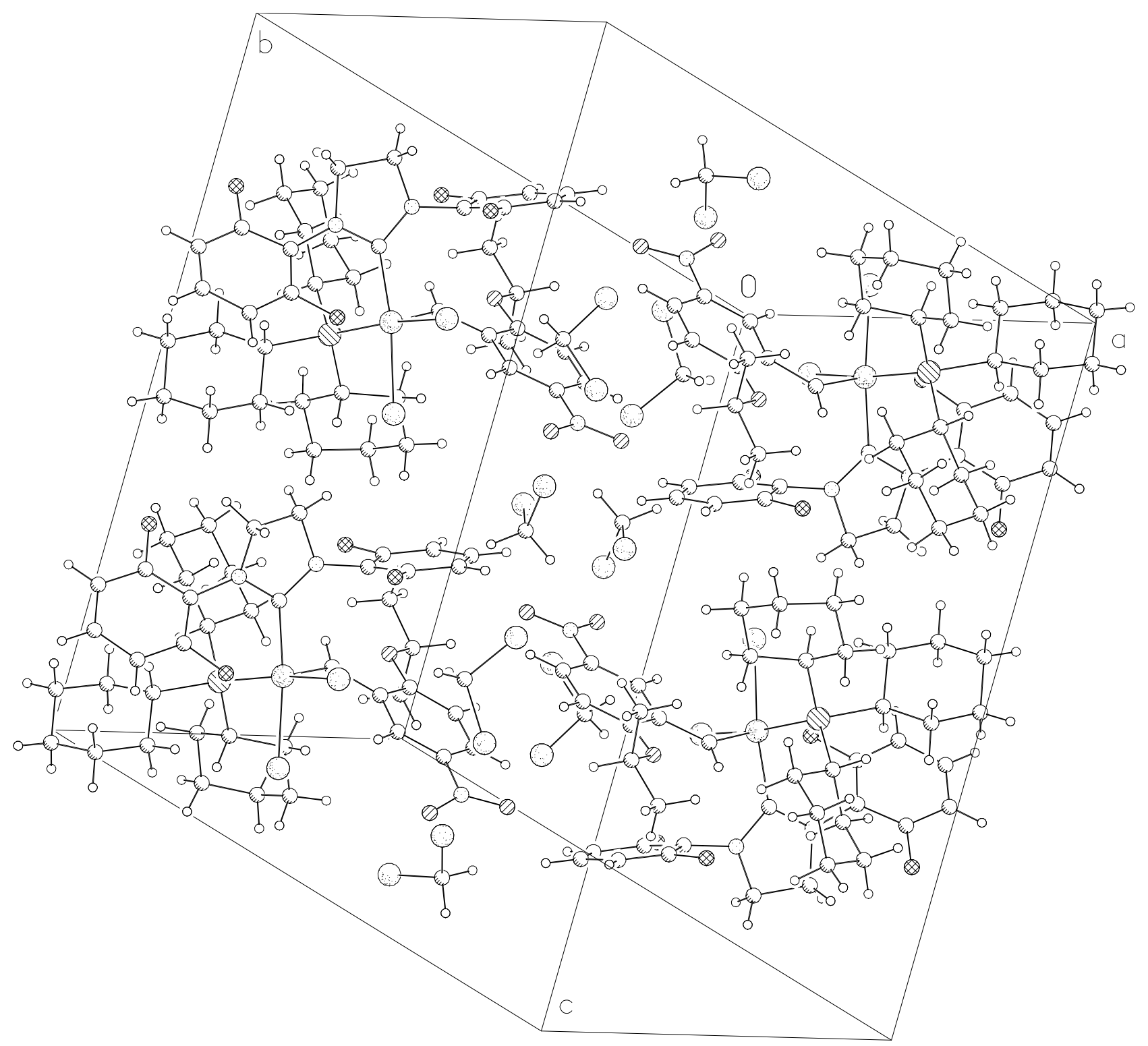

Figure 6. Unit cell packing. 

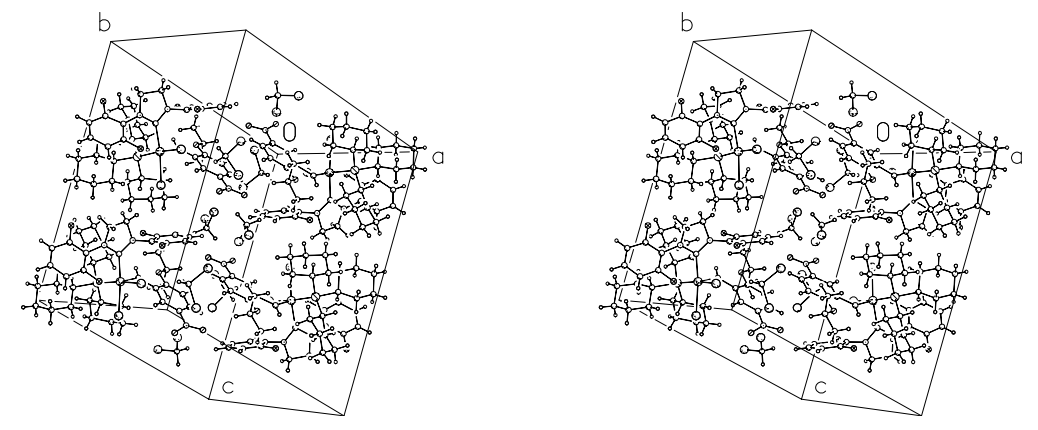

Figure 7. Stereo-view of unit cell packing. 
Table 2. Atomic coordinates $\left(\mathrm{x} \mathrm{10}^{4}\right)$ and equivalent isotropic displacement parameters $\left(\AA^{2} \times 10^{3}\right)$ for CCDC 284775 . U(eq) is defined as the trace of the orthogonalized $U^{i j}$ tensor.

\begin{tabular}{|c|c|c|c|c|}
\hline & $\mathrm{x}$ & $\mathrm{y}$ & $\mathrm{z}$ & $\mathrm{U}_{\text {eq }}$ \\
\hline $\mathrm{Ru}(1)$ & $6424(1)$ & $1677(1)$ & $6438(1)$ & 11(1) \\
\hline $\mathrm{Cl}(1 \mathrm{~A})$ & 4801(1) & $1636(1)$ & $6487(1)$ & $15(1)$ \\
\hline $\mathrm{Cl}(2 \mathrm{~A})$ & $6078(1)$ & 1964(1) & $5283(1)$ & $15(1)$ \\
\hline $\mathrm{P}(1)$ & $7956(1)$ & $1610(1)$ & $6218(1)$ & $12(1)$ \\
\hline $\mathrm{F}(1 \mathrm{~A})$ & 5613(1) & $220(1)$ & $5902(1)$ & $22(1)$ \\
\hline $\mathrm{F}(2 \mathrm{~A})$ & 7903(1) & $-785(1)$ & $7283(1)$ & $26(1)$ \\
\hline $\mathrm{F}(3 \mathrm{~A})$ & $4632(1)$ & $1759(1)$ & $8047(1)$ & $22(1)$ \\
\hline $\mathrm{F}(4 \mathrm{~A})$ & $7702(1)$ & $2710(1)$ & $8626(1)$ & $23(1)$ \\
\hline $\mathrm{O}(1 \mathrm{~A})$ & $3976(1)$ & $3498(1)$ & $5713(1)$ & $32(1)$ \\
\hline $\mathrm{O}(2 \mathrm{~A})$ & $3966(1)$ & $4762(1)$ & $6109(1)$ & $27(1)$ \\
\hline $\mathrm{O}(3 \mathrm{~A})$ & $7629(1)$ & $4102(1)$ & 7771(1) & $18(1)$ \\
\hline $\mathrm{N}(1 \mathrm{~A})$ & $6643(2)$ & $364(1)$ & $7240(1)$ & $13(1)$ \\
\hline $\mathrm{N}(2 \mathrm{~A})$ & 6421(1) & $1465(1)$ & $7958(1)$ & $13(1)$ \\
\hline $\mathrm{N}(3 \mathrm{~A})$ & $4321(2)$ & $4133(1)$ & $6088(1)$ & $21(1)$ \\
\hline$C(1 \mathrm{~A})$ & $6530(2)$ & $1154(2)$ & $7275(1)$ & $12(1)$ \\
\hline$C(2 A)$ & $6711(2)$ & $169(2)$ & $7952(1)$ & $20(1)$ \\
\hline$C(3 \mathrm{~A})$ & $6334(2)$ & $867(2)$ & $8392(1)$ & $18(1)$ \\
\hline $\mathrm{C}(4 \mathrm{~A})$ & $6717(2)$ & $-250(2)$ & $6643(1)$ & $14(1)$ \\
\hline$C(5 A)$ & $6155(2)$ & $-369(2)$ & 5992(1) & $16(1)$ \\
\hline$C(6 A)$ & $6121(2)$ & $-1018(2)$ & $5443(2)$ & $19(1)$ \\
\hline$C(7 A)$ & $6693(2)$ & $-1607(2)$ & $5527(2)$ & $22(1)$ \\
\hline $\mathrm{C}(8 \mathrm{~A})$ & $7295(2)$ & $-1514(2)$ & $6143(2)$ & $21(1)$ \\
\hline$C(9 A)$ & $7303(2)$ & $-849(2)$ & $6683(2)$ & $19(1)$ \\
\hline$C(10 A)$ & $6170(2)$ & $2230(2)$ & $8265(1)$ & $14(1)$ \\
\hline$C(11 \mathrm{~A})$ & $5266(2)$ & $2360(2)$ & $8339(1)$ & $16(1)$ \\
\hline
\end{tabular}




\begin{tabular}{|c|c|c|c|c|}
\hline$C(12 A)$ & $5009(2)$ & $3056(2)$ & $8722(2)$ & $20(1)$ \\
\hline$C(13 \mathrm{~A})$ & $5675(2)$ & $3649(2)$ & $9059(2)$ & $24(1)$ \\
\hline$C(14 A)$ & $6585(2)$ & $3552(2)$ & $9021(2)$ & $21(1)$ \\
\hline$C(15 A)$ & $6811(2)$ & $2837(2)$ & $8638(1)$ & $17(1)$ \\
\hline$C(16 A)$ & $6785(2)$ & 2673(2) & $7009(1)$ & $13(1)$ \\
\hline$C(17 A)$ & $6373(2)$ & $3415(2)$ & $6987(1)$ & $12(1)$ \\
\hline$C(18 \mathrm{~A})$ & $5552(2)$ & $3431(2)$ & $6550(1)$ & $15(1)$ \\
\hline$C(19 A)$ & $5193(2)$ & $4144(2)$ & $6541(1)$ & $16(1)$ \\
\hline$C(20 A)$ & $5618(2)$ & $4858(2)$ & $6939(2)$ & 19(1) \\
\hline$C(21 A)$ & $6437(2)$ & $4863(2)$ & $7361(2)$ & $18(1)$ \\
\hline$C(22 \mathrm{~A})$ & $6824(2)$ & $4150(2)$ & $7386(1)$ & $16(1)$ \\
\hline$C(23 A)$ & $8210(2)$ & $4816(2)$ & $8135(2)$ & $24(1)$ \\
\hline$C(24 A)$ & $8906(2)$ & $4552(2)$ & $8653(2)$ & $32(1)$ \\
\hline$C(25 \mathrm{~A})$ & $8647(2)$ & $5173(2)$ & $7605(2)$ & $30(1)$ \\
\hline$C(26 \mathrm{~A})$ & $8920(2)$ & $1702(2)$ & 6951(1) & $16(1)$ \\
\hline$C(27 A)$ & $8758(2)$ & 1162(2) & $7461(1)$ & $20(1)$ \\
\hline$C(28 \mathrm{~A})$ & $9629(2)$ & $1146(2)$ & $7969(2)$ & $29(1)$ \\
\hline$C(29 A)$ & $9995(2)$ & 1977(2) & $8383(2)$ & $35(1)$ \\
\hline$C(30 A)$ & $10144(2)$ & $2508(2)$ & $7880(2)$ & $29(1)$ \\
\hline$C(31 \mathrm{~A})$ & $9265(2)$ & $2542(2)$ & $7388(2)$ & $23(1)$ \\
\hline$C(32 \mathrm{~A})$ & $8324(2)$ & $2337(2)$ & $5706(1)$ & $15(1)$ \\
\hline$C(33 A)$ & $8089(2)$ & $3188(2)$ & $6004(2)$ & $18(1)$ \\
\hline$C(34 \mathrm{~A})$ & $8406(2)$ & $3772(2)$ & $5581(2)$ & $21(1)$ \\
\hline$C(35 A)$ & $9405(2)$ & $3738(2)$ & $5492(2)$ & $23(1)$ \\
\hline$C(36 \mathrm{~A})$ & $9571(2)$ & 2899(2) & $5128(2)$ & $22(1)$ \\
\hline$C(37 A)$ & $9318(2)$ & $2305(2)$ & $5560(2)$ & 19(1) \\
\hline$C(38 \mathrm{~A})$ & $8074(2)$ & $615(2)$ & $5628(1)$ & $14(1)$ \\
\hline$C(39 A)$ & $7722(2)$ & $586(2)$ & $4846(1)$ & $19(1)$ \\
\hline $\mathrm{C}(40 \mathrm{~A})$ & $7707(2)$ & $-246(2)$ & 4371(1) & $20(1)$ \\
\hline
\end{tabular}




\begin{tabular}{|c|c|c|c|c|}
\hline$C(41 \mathrm{~A})$ & $8640(2)$ & $-550(2)$ & $4438(1)$ & $19(1)$ \\
\hline $\mathrm{C}(42 \mathrm{~A})$ & $8969(2)$ & $-554(2)$ & $5210(1)$ & $16(1)$ \\
\hline$C(43 A)$ & $9001(2)$ & $266(2)$ & $5699(1)$ & $16(1)$ \\
\hline $\mathrm{Ru}(2)$ & $6314(1)$ & 1481(1) & $1420(1)$ & $10(1)$ \\
\hline $\mathrm{Cl}(1 \mathrm{~B})$ & $4680(1)$ & $1463(1)$ & $1392(1)$ & $15(1)$ \\
\hline $\mathrm{Cl}(2 \mathrm{~B})$ & $6099(1)$ & $1722(1)$ & $240(1)$ & $16(1)$ \\
\hline $\mathrm{P}(2)$ & $7876(1)$ & $1357(1)$ & $1276(1)$ & $12(1)$ \\
\hline $\mathrm{F}(1 \mathrm{~B})$ & $5552(1)$ & $8(1)$ & $851(1)$ & $20(1)$ \\
\hline $\mathrm{F}(2 \mathrm{~B})$ & $7560(1)$ & $-838(1)$ & $2554(1)$ & $19(1)$ \\
\hline $\mathrm{F}(3 \mathrm{~B})$ & $4283(1)$ & $1696(1)$ & $2918(1)$ & $22(1)$ \\
\hline $\mathrm{F}(4 \mathrm{~B})$ & $7358(1)$ & $2557(1)$ & $3692(1)$ & $24(1)$ \\
\hline $\mathrm{O}(1 \mathrm{~B})$ & $4149(1)$ & $3386(1)$ & $341(1)$ & $33(1)$ \\
\hline $\mathrm{O}(2 \mathrm{~B})$ & $3908(1)$ & $4556(1)$ & $919(1)$ & $33(1)$ \\
\hline $\mathrm{O}(3 \mathrm{~B})$ & $7460(1)$ & $3927(1)$ & $2744(1)$ & $18(1)$ \\
\hline $\mathrm{N}(1 \mathrm{~B})$ & $6331(1)$ & $232(1)$ & $2284(1)$ & $11(1)$ \\
\hline $\mathrm{N}(2 \mathrm{~B})$ & $6064(1)$ & 1352(1) & 2934(1) & $13(1)$ \\
\hline $\mathrm{N}(3 \mathrm{~B})$ & $4351(2)$ & $3968(1)$ & $837(1)$ & $23(1)$ \\
\hline$C(1 B)$ & $6282(2)$ & 1021(2) & $2282(1)$ & $11(1)$ \\
\hline $\mathrm{C}(2 \mathrm{~B})$ & $5922(2)$ & $6(2)$ & $2878(1)$ & $16(1)$ \\
\hline $\mathrm{C}(3 \mathrm{~B})$ & $6043(2)$ & 793(2) & $3412(1)$ & $17(1)$ \\
\hline$C(4 B)$ & $6524(2)$ & $-391(2)$ & $1728(1)$ & $12(1)$ \\
\hline $\mathrm{C}(5 \mathrm{~B})$ & $6092(2)$ & $-542(2)$ & $1032(1)$ & $13(1)$ \\
\hline$C(6 B)$ & $6192(2)$ & $-1207(2)$ & $524(1)$ & $16(1)$ \\
\hline $\mathrm{C}(7 \mathrm{~B})$ & $6777(2)$ & $-1743(2)$ & $702(2)$ & $17(1)$ \\
\hline $\mathrm{C}(8 \mathrm{~B})$ & $7253(2)$ & $-1609(2)$ & $1388(1)$ & $17(1)$ \\
\hline C (9B) & $7113(2)$ & $-949(2)$ & $1880(1)$ & $14(1)$ \\
\hline$C(10 B)$ & $5835(2)$ & $2129(2)$ & $3224(1)$ & $13(1)$ \\
\hline $\mathrm{C}(11 \mathrm{~B})$ & $4928(2)$ & 2283(2) & $3239(1)$ & $16(1)$ \\
\hline
\end{tabular}




\begin{tabular}{|c|c|c|c|c|}
\hline$C(12 B)$ & $4666(2)$ & 2990(2) & $3605(2)$ & $21(1)$ \\
\hline$C(13 B)$ & $5334(2)$ & $3560(2)$ & $3987(2)$ & $23(1)$ \\
\hline$C(14 B)$ & $6240(2)$ & $3430(2)$ & $4008(2)$ & $22(1)$ \\
\hline$C(15 B)$ & $6471(2)$ & $2715(2)$ & $3635(2)$ & $18(1)$ \\
\hline$C(16 B)$ & $6641(2)$ & 2494(2) & $1969(1)$ & $12(1)$ \\
\hline$C(17 B)$ & $6252(2)$ & $3233(2)$ & 1891(1) & $14(1)$ \\
\hline$C(18 B)$ & $5488(2)$ & $3248(2)$ & 1398(1) & $14(1)$ \\
\hline$C(19 B)$ & $5155(2)$ & $3969(2)$ & $1356(2)$ & $17(1)$ \\
\hline$C(20 B)$ & $5548(2)$ & $4680(2)$ & $1777(2)$ & $19(1)$ \\
\hline$C(21 B)$ & $6322(2)$ & $4678(2)$ & $2242(2)$ & $18(1)$ \\
\hline$C(22 B)$ & $6684(2)$ & $3970(2)$ & $2305(1)$ & $14(1)$ \\
\hline$C(23 B)$ & $8022(2)$ & $4646(2)$ & $3122(2)$ & $21(1)$ \\
\hline$C(24 B)$ & $8655(2)$ & $4395(2)$ & $3688(2)$ & $28(1)$ \\
\hline$C(25 B)$ & $8534(2)$ & $4980(2)$ & $2606(2)$ & $28(1)$ \\
\hline$C(26 B)$ & $8749(2)$ & $1415(2)$ & $2060(1)$ & $17(1)$ \\
\hline$C(27 B)$ & $8637(2)$ & $745(2)$ & $2446(1)$ & $20(1)$ \\
\hline$C(28 B)$ & $9487(2)$ & $740(2)$ & 2979(2) & $30(1)$ \\
\hline$C(29 B)$ & $9701(2)$ & 1521(2) & $3499(2)$ & $32(1)$ \\
\hline $\mathrm{C}(30 \mathrm{~B})$ & $9773(2)$ & $2210(2)$ & $3126(2)$ & $31(1)$ \\
\hline$C(31 B)$ & $8897(2)$ & 2193(2) & $2600(2)$ & $28(1)$ \\
\hline$C(32 B)$ & $8362(2)$ & $2100(2)$ & $822(1)$ & $15(1)$ \\
\hline$C(33 B)$ & $8040(2)$ & 2923(2) & $1017(2)$ & $22(1)$ \\
\hline$C(34 B)$ & $8367(2)$ & $3454(2)$ & $554(2)$ & $28(1)$ \\
\hline$C(35 B)$ & $9389(2)$ & $3509(2)$ & $578(2)$ & $26(1)$ \\
\hline$C(36 B)$ & $9723(2)$ & $2690(2)$ & $395(2)$ & $39(1)$ \\
\hline$C(37 B)$ & $9407(2)$ & $2168(2)$ & $877(2)$ & $32(1)$ \\
\hline$C(38 B)$ & $8044(2)$ & $363(2)$ & $700(1)$ & $13(1)$ \\
\hline$C(39 B)$ & $7593(2)$ & $252(2)$ & $-76(1)$ & $18(1)$ \\
\hline$C(40 B)$ & $7667(2)$ & $-577(2)$ & $-521(1)$ & $17(1)$ \\
\hline
\end{tabular}




\begin{tabular}{|c|c|c|c|c|}
\hline$C(41 B)$ & $8638(2)$ & $-799(2)$ & $-495(1)$ & $20(1)$ \\
\hline$C(42 B)$ & $9055(2)$ & $-724(2)$ & $278(1)$ & $21(1)$ \\
\hline$C(43 B)$ & $9029(2)$ & $123(2)$ & $710(2)$ & $18(1)$ \\
\hline $\mathrm{C}(1)$ & 7177(3) & $7482(2)$ & $8140(2)$ & $53(1)$ \\
\hline $\mathrm{Cl}(1)$ & 7531(1) & $7038(1)$ & $8842(1)$ & $58(1)$ \\
\hline $\mathrm{Cl}(2)$ & $7700(1)$ & $7115(1)$ & $7400(1)$ & $57(1)$ \\
\hline$C(2)$ & $7578(2)$ & $5761(2)$ & $5230(2)$ & $36(1)$ \\
\hline $\mathrm{Cl}(3)$ & $6795(1)$ & $5387(1)$ & $5703(1)$ & $36(1)$ \\
\hline $\mathrm{Cl}(4)$ & $8575(1)$ & $6202(1)$ & 5771(1) & $56(1)$ \\
\hline$C(3)$ & $7236(2)$ & $7490(2)$ & $3402(2)$ & $31(1)$ \\
\hline $\mathrm{Cl}(5)$ & $8101(1)$ & $7195(1)$ & $3974(1)$ & $41(1)$ \\
\hline $\mathrm{Cl}(6)$ & 7051(1) & $6829(1)$ & $2557(1)$ & $51(1)$ \\
\hline $\mathrm{C}(4)$ & $7664(2)$ & $6077(3)$ & $523(3)$ & $75(2)$ \\
\hline $\mathrm{Cl}(7)$ & $6965(1)$ & $5448(1)$ & $851(1)$ & $47(1)$ \\
\hline $\mathrm{Cl}(8)$ & $8710(1)$ & $5787(1)$ & $430(1)$ & $102(1)$ \\
\hline
\end{tabular}


Table 3. Selected bond lengths $[\AA \AA]$ and angles $\left[{ }^{\circ}\right]$ for CCDC 284775.

\begin{tabular}{llll}
\hline $\mathrm{Ru}(1)-\mathrm{C}(16 \mathrm{~A})$ & $1.846(3)$ & $\mathrm{Ru}(2)-\mathrm{C}(16 \mathrm{~B})$ & $1.849(2)$ \\
$\mathrm{Ru}(1)-\mathrm{C}(1 \mathrm{~A})$ & $2.024(3)$ & $\mathrm{Ru}(2)-\mathrm{C}(1 \mathrm{~B})$ & $2.011(3)$ \\
$\mathrm{Ru}(1)-\mathrm{P}(1)$ & $2.3723(7)$ & $\mathrm{Ru}(2)-\mathrm{P}(2)$ & $2.3887(7)$ \\
$\mathrm{Ru}(1)-\mathrm{Cl}(2 \mathrm{~A})$ & $2.4046(7)$ & $\mathrm{Ru}(2)-\mathrm{Cl}(2 \mathrm{~B})$ & $2.4075(7)$ \\
$\mathrm{Ru}(1)-\mathrm{Cl}(1 \mathrm{~A})$ & $2.4230(7)$ & $\mathrm{Ru}(2)-\mathrm{Cl}(1 \mathrm{~B})$ & $2.4183(7)$
\end{tabular}

None-bonded distances

$\mathrm{Ru}(1)-\mathrm{F}(1 \mathrm{~A})$

2.6677(15)

$\mathrm{Ru}(2)-\mathrm{F}(1 \mathrm{~B})$

2.6748(15)

$\begin{array}{lrlr}\mathrm{C}(16 \mathrm{~A})-\mathrm{Ru}(1)-\mathrm{C}(1 \mathrm{~A}) & 92.92(11) & \mathrm{C}(16 \mathrm{~B})-\mathrm{Ru}(2)-\mathrm{C}(1 \mathrm{~B}) & 92.57(10) \\ \mathrm{C}(16 \mathrm{~A})-\mathrm{Ru}(1)-\mathrm{P}(1) & 86.93(8) & \mathrm{C}(16 \mathrm{~B})-\mathrm{Ru}(2)-\mathrm{P}(2) & 88.70(8) \\ \mathrm{C}(1 \mathrm{~A})-\mathrm{Ru}(1)-\mathrm{P}(1) & 97.43(7) & \mathrm{C}(1 \mathrm{~B})-\mathrm{Ru}(2)-\mathrm{P}(2) & 99.23(7) \\ \mathrm{C}(16 \mathrm{~A})-\mathrm{Ru}(1)-\mathrm{Cl}(2 \mathrm{~A}) & 101.99(8) & \mathrm{C}(16 \mathrm{~B})-\mathrm{Ru}(2)-\mathrm{Cl}(2 \mathrm{~B}) & 101.36(8) \\ \mathrm{C}(1 \mathrm{~A})-\mathrm{Ru}(1)-\mathrm{Cl}(2 \mathrm{~A}) & 164.63(7) & \mathrm{C}(1 \mathrm{~B})-\mathrm{Ru}(2)-\mathrm{Cl}(2 \mathrm{~B}) & 165.02(7) \\ \mathrm{P}(1)-\mathrm{Ru}(1)-\mathrm{Cl}(2 \mathrm{~A}) & 87.21(2) & \mathrm{P}(2)-\mathrm{Ru}(2)-\mathrm{Cl}(2 \mathrm{~B}) & 86.67(2) \\ \mathrm{C}(16 \mathrm{~A})-\mathrm{Ru}(1)-\mathrm{Cl}(1 \mathrm{~A}) & 99.93(8) & \mathrm{C}(16 \mathrm{~B})-\mathrm{Ru}(2)-\mathrm{Cl}(1 \mathrm{~B}) & 99.02(8) \\ \mathrm{C}(1 \mathrm{~A})-\mathrm{Ru}(1)-\mathrm{Cl}(1 \mathrm{~A}) & 86.94(7) & \mathrm{C}(1 \mathrm{~B})-\mathrm{Ru}(2)-\mathrm{Cl}(1 \mathrm{~B}) & 84.60(7) \\ \mathrm{P}(1)-\mathrm{Ru}(1)-\mathrm{Cl}(1 \mathrm{~A}) & 171.71(3) & \mathrm{P}(2)-\mathrm{Ru}(2)-\mathrm{Cl}(1 \mathrm{~B}) & 171.26(3) \\ \mathrm{Cl}(2 \mathrm{~A})-\mathrm{Ru}(1)-\mathrm{Cl}(1 \mathrm{~A}) & 86.80(2) & \mathrm{Cl}(2 \mathrm{~B})-\mathrm{Ru}(2)-\mathrm{Cl}(1 \mathrm{~B}) & 87.78(2)\end{array}$

Non-bonded angles

$\mathrm{F}(1 \mathrm{~A})-\mathrm{Ru}(1)-\mathrm{C}(1 \mathrm{~A})$

75.71(8)

$\mathrm{F}(1 \mathrm{~A})-\mathrm{Ru}(1)-\mathrm{P}(1)$

105.83(4) 


\begin{tabular}{lrlr}
$\mathrm{F}(1 \mathrm{~A})-\mathrm{Ru}(1)-\mathrm{C}(16 \mathrm{~A})$ & $163.73(8)$ & $\mathrm{F}(1 \mathrm{~B})-\mathrm{Ru}(2)-\mathrm{P}(2)$ & $102.54(4)$ \\
$\mathrm{F}(1 \mathrm{~A})-\mathrm{Ru}(1)-\mathrm{Cl}(1 \mathrm{~A})$ & $68.33(4)$ & $\mathrm{F}(1 \mathrm{~B})-\mathrm{Ru}(2)-\mathrm{C}(16 \mathrm{~B})$ & $166.01(8)$ \\
$\mathrm{F}(1 \mathrm{~A})-\mathrm{Ru}(1)-\mathrm{Cl}(2 \mathrm{~A})$ & $88.93(4)$ & $\mathrm{F}(1 \mathrm{~B})-\mathrm{Ru}(2)-\mathrm{Cl}(1 \mathrm{~B})$ & $70.47(4)$ \\
$\mathrm{F}(1 \mathrm{~B})-\mathrm{Ru}(2)-\mathrm{C}(1 \mathrm{~B})$ & $77.57(8)$ & $\mathrm{F}(1 \mathrm{~B})-\mathrm{Ru}(2)-\mathrm{Cl}(2 \mathrm{~B})$ & $87.74(4)$ \\
& & & \\
\hline
\end{tabular}


Table 4. Bond lengths $[\AA \AA]$ and angles $\left[^{\circ}\right]$ for CCDC 284775.

\begin{tabular}{|c|c|c|c|}
\hline $\mathrm{Ru}(1)-\mathrm{C}(16 \mathrm{~A})$ & $1.846(3)$ & $C(5 A)-C(6 A)$ & $1.366(4)$ \\
\hline $\mathrm{Ru}(1)-\mathrm{C}(1 \mathrm{~A})$ & $2.024(3)$ & $C(6 A)-C(7 A)$ & $1.387(4)$ \\
\hline $\mathrm{Ru}(1)-\mathrm{P}(1)$ & $2.3723(7)$ & $\mathrm{C}(7 \mathrm{~A})-\mathrm{C}(8 \mathrm{~A})$ & $1.373(4)$ \\
\hline $\mathrm{Ru}(1)-\mathrm{Cl}(2 \mathrm{~A})$ & $2.4046(7)$ & $\mathrm{C}(8 \mathrm{~A})-\mathrm{C}(9 \mathrm{~A})$ & $1.377(4)$ \\
\hline $\mathrm{Ru}(1)-\mathrm{Cl}(1 \mathrm{~A})$ & $2.4230(7)$ & $\mathrm{C}(10 \mathrm{~A})-\mathrm{C}(15 \mathrm{~A})$ & $1.387(4)$ \\
\hline $\mathrm{Ru}(1)-\mathrm{F}(1 \mathrm{~A})$ & $2.6677(15)$ & $C(10 A)-C(11 A)$ & $1.394(4)$ \\
\hline$P(1)-C(26 A)$ & $1.864(3)$ & $\mathrm{C}(11 \mathrm{~A})-\mathrm{C}(12 \mathrm{~A})$ & $1.372(4)$ \\
\hline $\mathrm{P}(1)-\mathrm{C}(38 \mathrm{~A})$ & $1.879(3)$ & $C(12 A)-C(13 A)$ & $1.377(4)$ \\
\hline$P(1)-C(32 A)$ & $1.869(3)$ & $\mathrm{C}(13 \mathrm{~A})-\mathrm{C}(14 \mathrm{~A})$ & $1.381(4)$ \\
\hline $\mathrm{F}(1 \mathrm{~A})-\mathrm{C}(5 \mathrm{~A})$ & $1.361(3)$ & $\mathrm{C}(14 \mathrm{~A})-\mathrm{C}(15 \mathrm{~A})$ & $1.380(4)$ \\
\hline $\mathrm{F}(2 \mathrm{~A})-\mathrm{C}(9 \mathrm{~A})$ & $1.356(3)$ & $C(16 A)-C(17 A)$ & $1.460(3)$ \\
\hline $\mathrm{F}(3 \mathrm{~A})-\mathrm{C}(11 \mathrm{~A})$ & $1.340(3)$ & $\mathrm{C}(17 \mathrm{~A})-\mathrm{C}(18 \mathrm{~A})$ & $1.400(3)$ \\
\hline $\mathrm{F}(4 \mathrm{~A})-\mathrm{C}(15 \mathrm{~A})$ & $1.356(3)$ & $\mathrm{C}(17 \mathrm{~A})-\mathrm{C}(22 \mathrm{~A})$ & $1.421(4)$ \\
\hline $\mathrm{O}(1 \mathrm{~A})-\mathrm{N}(3 \mathrm{~A})$ & $1.224(3)$ & $\mathrm{C}(18 \mathrm{~A})-\mathrm{C}(19 \mathrm{~A})$ & $1.376(4)$ \\
\hline $\mathrm{O}(2 \mathrm{~A})-\mathrm{N}(3 \mathrm{~A})$ & $1.231(3)$ & $C(19 A)-C(20 A)$ & $1.380(4)$ \\
\hline $\mathrm{O}(3 \mathrm{~A})-\mathrm{C}(22 \mathrm{~A})$ & $1.345(3)$ & $C(20 A)-C(21 A)$ & $1.376(4)$ \\
\hline $\mathrm{O}(3 \mathrm{~A})-\mathrm{C}(23 \mathrm{~A})$ & $1.458(3)$ & $\mathrm{C}(21 \mathrm{~A})-\mathrm{C}(22 \mathrm{~A})$ & $1.399(4)$ \\
\hline $\mathrm{N}(1 \mathrm{~A})-\mathrm{C}(1 \mathrm{~A})$ & $1.378(3)$ & $C(23 \mathrm{~A})-\mathrm{C}(25 \mathrm{~A})$ & $1.509(4)$ \\
\hline $\mathrm{N}(1 \mathrm{~A})-\mathrm{C}(4 \mathrm{~A})$ & $1.412(3)$ & $\mathrm{C}(23 \mathrm{~A})-\mathrm{C}(24 \mathrm{~A})$ & $1.511(4)$ \\
\hline $\mathrm{N}(1 \mathrm{~A})-\mathrm{C}(2 \mathrm{~A})$ & $1.487(3)$ & $C(26 \mathrm{~A})-\mathrm{C}(27 \mathrm{~A})$ & $1.536(3)$ \\
\hline $\mathrm{N}(2 \mathrm{~A})-\mathrm{C}(1 \mathrm{~A})$ & $1.352(3)$ & $C(26 A)-C(31 A)$ & $1.540(4)$ \\
\hline $\mathrm{N}(2 \mathrm{~A})-\mathrm{C}(10 \mathrm{~A})$ & $1.421(3)$ & $\mathrm{C}(27 \mathrm{~A})-\mathrm{C}(28 \mathrm{~A})$ & $1.531(4)$ \\
\hline $\mathrm{N}(2 \mathrm{~A})-\mathrm{C}(3 \mathrm{~A})$ & $1.483(3)$ & $C(28 \mathrm{~A})-\mathrm{C}(29 \mathrm{~A})$ & $1.521(4)$ \\
\hline $\mathrm{N}(3 \mathrm{~A})-\mathrm{C}(19 \mathrm{~A})$ & $1.468(3)$ & $C(29 A)-C(30 A)$ & $1.511(4)$ \\
\hline$C(2 A)-C(3 A)$ & $1.501(4)$ & $C(30 A)-C(31 A)$ & $1.530(4)$ \\
\hline$C(4 A)-C(5 A)$ & $1.393(4)$ & $\mathrm{C}(32 \mathrm{~A})-\mathrm{C}(37 \mathrm{~A})$ & $1.539(3)$ \\
\hline$C(4 A)-C(9 A)$ & $1.399(4)$ & $\mathrm{C}(32 \mathrm{~A})-\mathrm{C}(33 \mathrm{~A})$ & $1.539(4)$ \\
\hline
\end{tabular}




\begin{tabular}{|c|c|c|c|}
\hline$C(33 A)-C(34 A)$ & $1.528(3)$ & $\mathrm{N}(1 \mathrm{~B})-\mathrm{C}(2 \mathrm{~B})$ & $1.485(3)$ \\
\hline $\mathrm{C}(34 \mathrm{~A})-\mathrm{C}(35 \mathrm{~A})$ & $1.519(4)$ & $\mathrm{N}(2 \mathrm{~B})-\mathrm{C}(1 \mathrm{~B})$ & $1.358(3)$ \\
\hline$C(35 A)-C(36 A)$ & $1.520(4)$ & $\mathrm{N}(2 \mathrm{~B})-\mathrm{C}(10 \mathrm{~B})$ & $1.420(3)$ \\
\hline$C(36 \mathrm{~A})-\mathrm{C}(37 \mathrm{~A})$ & $1.525(3)$ & $\mathrm{N}(2 \mathrm{~B})-\mathrm{C}(3 \mathrm{~B})$ & $1.484(3)$ \\
\hline$C(38 \mathrm{~A})-\mathrm{C}(43 \mathrm{~A})$ & $1.536(3)$ & $\mathrm{N}(3 \mathrm{~B})-\mathrm{C}(19 \mathrm{~B})$ & $1.462(3)$ \\
\hline $\mathrm{C}(38 \mathrm{~A})-\mathrm{C}(39 \mathrm{~A})$ & $1.533(3)$ & $\mathrm{C}(2 \mathrm{~B})-\mathrm{C}(3 \mathrm{~B})$ & $1.509(3)$ \\
\hline$C(39 A)-C(40 A)$ & $1.529(4)$ & $\mathrm{C}(4 \mathrm{~B})-\mathrm{C}(5 \mathrm{~B})$ & $1.384(3)$ \\
\hline$C(40 A)-C(41 A)$ & $1.509(4)$ & $\mathrm{C}(4 \mathrm{~B})-\mathrm{C}(9 \mathrm{~B})$ & $1.393(3)$ \\
\hline$C(41 \mathrm{~A})-\mathrm{C}(42 \mathrm{~A})$ & $1.520(4)$ & $\mathrm{C}(5 \mathrm{~B})-\mathrm{C}(6 \mathrm{~B})$ & $1.373(3)$ \\
\hline$C(42 A)-C(43 A)$ & $1.522(3)$ & $\mathrm{C}(6 \mathrm{~B})-\mathrm{C}(7 \mathrm{~B})$ & $1.377(4)$ \\
\hline $\mathrm{Ru}(2)-\mathrm{C}(16 \mathrm{~B})$ & $1.849(2)$ & $\mathrm{C}(7 \mathrm{~B})-\mathrm{C}(8 \mathrm{~B})$ & $1.392(4)$ \\
\hline $\mathrm{Ru}(2)-\mathrm{C}(1 \mathrm{~B})$ & $2.011(3)$ & $\mathrm{C}(8 \mathrm{~B})-\mathrm{C}(9 \mathrm{~B})$ & $1.366(3)$ \\
\hline $\mathrm{Ru}(2)-\mathrm{P}(2)$ & $2.3887(7)$ & $C(10 B)-C(15 B)$ & $1.383(4)$ \\
\hline $\mathrm{Ru}(2)-\mathrm{Cl}(2 \mathrm{~B})$ & $2.4075(7)$ & $\mathrm{C}(10 \mathrm{~B})-\mathrm{C}(11 \mathrm{~B})$ & $1.389(4)$ \\
\hline $\mathrm{Ru}(2)-\mathrm{Cl}(1 \mathrm{~B})$ & $2.4183(7)$ & $\mathrm{C}(11 \mathrm{~B})-\mathrm{C}(12 \mathrm{~B})$ & $1.378(4)$ \\
\hline $\mathrm{Ru}(2)-\mathrm{F}(1 \mathrm{~B})$ & $2.6748(15)$ & $\mathrm{C}(12 \mathrm{~B})-\mathrm{C}(13 \mathrm{~B})$ & $1.379(4)$ \\
\hline $\mathrm{P}(2)-\mathrm{C}(26 \mathrm{~B})$ & $1.853(3)$ & $C(13 B)-C(14 B)$ & $1.374(4)$ \\
\hline $\mathrm{P}(2)-\mathrm{C}(32 \mathrm{~B})$ & $1.877(3)$ & $\mathrm{C}(14 \mathrm{~B})-\mathrm{C}(15 \mathrm{~B})$ & $1.376(4)$ \\
\hline $\mathrm{P}(2)-\mathrm{C}(38 \mathrm{~B})$ & $1.885(3)$ & $C(16 B)-C(17 B)$ & $1.462(3)$ \\
\hline $\mathrm{F}(1 \mathrm{~B})-\mathrm{C}(5 \mathrm{~B})$ & $1.355(3)$ & $\mathrm{C}(17 \mathrm{~B})-\mathrm{C}(18 \mathrm{~B})$ & $1.396(3)$ \\
\hline $\mathrm{F}(2 \mathrm{~B})-\mathrm{C}(9 \mathrm{~B})$ & $1.360(3)$ & $\mathrm{C}(17 \mathrm{~B})-\mathrm{C}(22 \mathrm{~B})$ & $1.423(4)$ \\
\hline$F(3 B)-C(11 B)$ & $1.345(3)$ & $\mathrm{C}(18 \mathrm{~B})-\mathrm{C}(19 \mathrm{~B})$ & $1.384(3)$ \\
\hline $\mathrm{F}(4 \mathrm{~B})-\mathrm{C}(15 \mathrm{~B})$ & $1.357(3)$ & $C(19 B)-C(20 B)$ & $1.376(4)$ \\
\hline $\mathrm{O}(1 \mathrm{~B})-\mathrm{N}(3 \mathrm{~B})$ & $1.227(3)$ & $C(20 B)-C(21 B)$ & $1.371(4)$ \\
\hline $\mathrm{O}(2 \mathrm{~B})-\mathrm{N}(3 \mathrm{~B})$ & $1.236(3)$ & $C(21 B)-C(22 B)$ & $1.390(4)$ \\
\hline $\mathrm{O}(3 \mathrm{~B})-\mathrm{C}(22 \mathrm{~B})$ & $1.360(3)$ & $C(23 B)-C(24 B)$ & $1.509(4)$ \\
\hline $\mathrm{O}(3 \mathrm{~B})-\mathrm{C}(23 \mathrm{~B})$ & $1.457(3)$ & $C(23 B)-C(25 B)$ & $1.524(4)$ \\
\hline $\mathrm{N}(1 \mathrm{~B})-\mathrm{C}(1 \mathrm{~B})$ & $1.374(3)$ & $C(26 B)-C(31 B)$ & $1.501(4)$ \\
\hline $\mathrm{N}(1 \mathrm{~B})-\mathrm{C}(4 \mathrm{~B})$ & $1.420(3)$ & $\mathrm{C}(26 \mathrm{~B})-\mathrm{C}(27 \mathrm{~B})$ & $1.528(3)$ \\
\hline
\end{tabular}




\begin{tabular}{|c|c|c|c|}
\hline $\mathrm{C}(27 \mathrm{~B})-\mathrm{C}(28 \mathrm{~B})$ & $1.530(4)$ & $\mathrm{C}(1 \mathrm{~A})-\mathrm{Ru}(1)-\mathrm{Cl}(2 \mathrm{~A})$ & $164.63(7)$ \\
\hline $\mathrm{C}(28 \mathrm{~B})-\mathrm{C}(29 \mathrm{~B})$ & $1.493(4)$ & $\mathrm{P}(1)-\mathrm{Ru}(1)-\mathrm{Cl}(2 \mathrm{~A})$ & $87.21(2)$ \\
\hline $\mathrm{C}(29 \mathrm{~B})-\mathrm{C}(30 \mathrm{~B})$ & $1.532(4)$ & $C(16 \mathrm{~A})-\mathrm{Ru}(1)-\mathrm{Cl}(1 \mathrm{~A})$ & $99.93(8)$ \\
\hline $\mathrm{C}(30 \mathrm{~B})-\mathrm{C}(31 \mathrm{~B})$ & $1.546(4)$ & $\mathrm{C}(1 \mathrm{~A})-\mathrm{Ru}(1)-\mathrm{Cl}(1 \mathrm{~A})$ & $86.94(7)$ \\
\hline$C(32 B)-C(33 B)$ & $1.514(4)$ & $\mathrm{P}(1)-\mathrm{Ru}(1)-\mathrm{Cl}(1 \mathrm{~A})$ & $171.71(3)$ \\
\hline $\mathrm{C}(32 \mathrm{~B})-\mathrm{C}(37 \mathrm{~B})$ & $1.539(4)$ & $\mathrm{Cl}(2 \mathrm{~A})-\mathrm{Ru}(1)-\mathrm{Cl}(1 \mathrm{~A})$ & $86.80(2)$ \\
\hline$C(33 B)-C(34 B)$ & $1.523(4)$ & $\mathrm{F}(1 \mathrm{~A})-\mathrm{Ru}(1)-\mathrm{C}(1 \mathrm{~A})$ & $75.71(8)$ \\
\hline$C(34 B)-C(35 B)$ & $1.509(4)$ & $\mathrm{F}(1 \mathrm{~A})-\mathrm{Ru}(1)-\mathrm{P}(1)$ & $105.83(4)$ \\
\hline$C(35 B)-C(36 B)$ & $1.513(4)$ & $F(1 A)-R u(1)-C(16 A)$ & $163.73(8)$ \\
\hline$C(36 \mathrm{~B})-\mathrm{C}(37 \mathrm{~B})$ & $1.534(4)$ & $\mathrm{F}(1 \mathrm{~A})-\mathrm{Ru}(1)-\mathrm{Cl}(1 \mathrm{~A})$ & $68.33(4)$ \\
\hline $\mathrm{C}(38 \mathrm{~B})-\mathrm{C}(43 \mathrm{~B})$ & $1.542(3)$ & $\mathrm{F}(1 \mathrm{~A})-\mathrm{Ru}(1)-\mathrm{Cl}(2 \mathrm{~A})$ & $88.93(4)$ \\
\hline C(38B)-C(39B) & $1.538(3)$ & $\mathrm{C}(26 \mathrm{~A})-\mathrm{P}(1)-\mathrm{C}(38 \mathrm{~A})$ & $102.72(12)$ \\
\hline C(39B)-C(40B) & $1.524(3)$ & $\mathrm{C}(26 \mathrm{~A})-\mathrm{P}(1)-\mathrm{C}(32 \mathrm{~A})$ & $103.53(12)$ \\
\hline$C(40 B)-C(41 B)$ & $1.510(4)$ & $\mathrm{C}(38 \mathrm{~A})-\mathrm{P}(1)-\mathrm{C}(32 \mathrm{~A})$ & $104.09(12)$ \\
\hline $\mathrm{C}(41 \mathrm{~B})-\mathrm{C}(42 \mathrm{~B})$ & $1.526(4)$ & $\mathrm{C}(26 \mathrm{~A})-\mathrm{P}(1)-\mathrm{Ru}(1)$ & $121.95(9)$ \\
\hline$C(42 B)-C(43 B)$ & $1.529(4)$ & $\mathrm{C}(38 \mathrm{~A})-\mathrm{P}(1)-\mathrm{Ru}(1)$ & $109.03(8)$ \\
\hline $\mathrm{C}(1)-\mathrm{Cl}(2)$ & $1.736(4)$ & $\mathrm{C}(32 \mathrm{~A})-\mathrm{P}(1)-\mathrm{Ru}(1)$ & $113.68(9)$ \\
\hline $\mathrm{C}(1)-\mathrm{Cl}(1)$ & $1.748(4)$ & $\mathrm{C}(22 \mathrm{~A})-\mathrm{O}(3 \mathrm{~A})-\mathrm{C}(23 \mathrm{~A})$ & $120.8(2)$ \\
\hline $\mathrm{C}(2)-\mathrm{Cl}(4)$ & $1.737(3)$ & $\mathrm{C}(1 \mathrm{~A})-\mathrm{N}(1 \mathrm{~A})-\mathrm{C}(4 \mathrm{~A})$ & $129.3(2)$ \\
\hline $\mathrm{C}(2)-\mathrm{Cl}(3)$ & $1.753(3)$ & $\mathrm{C}(1 \mathrm{~A})-\mathrm{N}(1 \mathrm{~A})-\mathrm{C}(2 \mathrm{~A})$ & $112.3(2)$ \\
\hline $\mathrm{C}(3)-\mathrm{Cl}(6)$ & $1.765(3)$ & $\mathrm{C}(4 \mathrm{~A})-\mathrm{N}(1 \mathrm{~A})-\mathrm{C}(2 \mathrm{~A})$ & $118.3(2)$ \\
\hline $\mathrm{C}(3)-\mathrm{Cl}(5)$ & $1.764(3)$ & $C(1 \mathrm{~A})-\mathrm{N}(2 \mathrm{~A})-\mathrm{C}(10 \mathrm{~A})$ & $129.0(2)$ \\
\hline $\mathrm{C}(4)-\mathrm{Cl}(8)$ & $1.678(4)$ & $\mathrm{C}(1 \mathrm{~A})-\mathrm{N}(2 \mathrm{~A})-\mathrm{C}(3 \mathrm{~A})$ & $114.1(2)$ \\
\hline \multirow[t]{2}{*}{$\mathrm{C}(4)-\mathrm{Cl}(7)$} & $1.739(4)$ & $C(10 A)-N(2 A)-C(3 A)$ & $115.0(2)$ \\
\hline & & $\mathrm{O}(1 \mathrm{~A})-\mathrm{N}(3 \mathrm{~A})-\mathrm{O}(2 \mathrm{~A})$ & $123.1(2)$ \\
\hline$C(16 A)-R u(1)-C(1 A)$ & $92.92(11)$ & $\mathrm{O}(1 \mathrm{~A})-\mathrm{N}(3 \mathrm{~A})-\mathrm{C}(19 \mathrm{~A})$ & $118.5(2)$ \\
\hline$C(16 A)-R u(1)-P(1)$ & $86.93(8)$ & $\mathrm{O}(2 \mathrm{~A})-\mathrm{N}(3 \mathrm{~A})-\mathrm{C}(19 \mathrm{~A})$ & $118.4(2)$ \\
\hline $\mathrm{C}(1 \mathrm{~A})-\mathrm{Ru}(1)-\mathrm{P}(1)$ & $97.43(7)$ & $\mathrm{N}(2 \mathrm{~A})-\mathrm{C}(1 \mathrm{~A})-\mathrm{N}(1 \mathrm{~A})$ & $105.4(2)$ \\
\hline $\mathrm{C}(16 \mathrm{~A})-\mathrm{Ru}(1)-\mathrm{Cl}(2 \mathrm{~A})$ & $101.99(8)$ & $\mathrm{N}(2 \mathrm{~A})-\mathrm{C}(1 \mathrm{~A})-\mathrm{Ru}(1)$ & $128.75(19)$ \\
\hline
\end{tabular}




\begin{tabular}{|c|c|c|c|}
\hline $\mathrm{N}(1 \mathrm{~A})-\mathrm{C}(1 \mathrm{~A})-\mathrm{Ru}(1)$ & $125.58(19)$ & $C(18 A)-C(17 A)-C(16 A)$ & $121.6(2)$ \\
\hline $\mathrm{N}(1 \mathrm{~A})-\mathrm{C}(2 \mathrm{~A})-\mathrm{C}(3 \mathrm{~A})$ & $102.5(2)$ & $C(22 A)-C(17 A)-C(16 A)$ & $120.3(2)$ \\
\hline $\mathrm{N}(2 \mathrm{~A})-\mathrm{C}(3 \mathrm{~A})-\mathrm{C}(2 \mathrm{~A})$ & $101.4(2)$ & $C(19 A)-C(18 A)-C(17 A)$ & $119.7(2)$ \\
\hline $\mathrm{C}(5 \mathrm{~A})-\mathrm{C}(4 \mathrm{~A})-\mathrm{N}(1 \mathrm{~A})$ & $123.9(2)$ & $\mathrm{C}(18 \mathrm{~A})-\mathrm{C}(19 \mathrm{~A})-\mathrm{C}(20 \mathrm{~A})$ & $122.5(3)$ \\
\hline$C(5 A)-C(4 A)-C(9 A)$ & $113.8(2)$ & $\mathrm{C}(18 \mathrm{~A})-\mathrm{C}(19 \mathrm{~A})-\mathrm{N}(3 \mathrm{~A})$ & $118.0(2)$ \\
\hline $\mathrm{N}(1 \mathrm{~A})-\mathrm{C}(4 \mathrm{~A})-\mathrm{C}(9 \mathrm{~A})$ & $122.0(2)$ & $C(20 A)-C(19 A)-N(3 A)$ & $119.5(2)$ \\
\hline $\mathrm{F}(1 \mathrm{~A})-\mathrm{C}(5 \mathrm{~A})-\mathrm{C}(4 \mathrm{~A})$ & $116.6(2)$ & $C(21 A)-C(20 A)-C(19 A)$ & $119.2(3)$ \\
\hline $\mathrm{F}(1 \mathrm{~A})-\mathrm{C}(5 \mathrm{~A})-\mathrm{C}(6 \mathrm{~A})$ & $118.3(2)$ & $C(20 A)-C(21 A)-C(22 A)$ & $120.0(3)$ \\
\hline$C(4 A)-C(5 A)-C(6 A)$ & $125.1(3)$ & $\mathrm{O}(3 \mathrm{~A})-\mathrm{C}(22 \mathrm{~A})-\mathrm{C}(21 \mathrm{~A})$ & $124.0(2)$ \\
\hline$C(5 A)-C(6 A)-C(7 A)$ & $118.2(3)$ & $\mathrm{O}(3 \mathrm{~A})-\mathrm{C}(22 \mathrm{~A})-\mathrm{C}(17 \mathrm{~A})$ & $115.4(2)$ \\
\hline$C(6 A)-C(7 A)-C(8 A)$ & $119.9(3)$ & $C(21 A)-C(22 A)-C(17 A)$ & $120.6(3)$ \\
\hline $\mathrm{C}(9 \mathrm{~A})-\mathrm{C}(8 \mathrm{~A})-\mathrm{C}(7 \mathrm{~A})$ & $119.8(3)$ & $\mathrm{O}(3 \mathrm{~A})-\mathrm{C}(23 \mathrm{~A})-\mathrm{C}(25 \mathrm{~A})$ & $110.3(2)$ \\
\hline $\mathrm{F}(2 \mathrm{~A})-\mathrm{C}(9 \mathrm{~A})-\mathrm{C}(8 \mathrm{~A})$ & $117.7(2)$ & $\mathrm{O}(3 \mathrm{~A})-\mathrm{C}(23 \mathrm{~A})-\mathrm{C}(24 \mathrm{~A})$ & $105.4(2)$ \\
\hline $\mathrm{F}(2 \mathrm{~A})-\mathrm{C}(9 \mathrm{~A})-\mathrm{C}(4 \mathrm{~A})$ & $119.1(2)$ & $C(25 A)-C(23 A)-C(24 A)$ & $112.1(3)$ \\
\hline $\mathrm{C}(8 \mathrm{~A})-\mathrm{C}(9 \mathrm{~A})-\mathrm{C}(4 \mathrm{~A})$ & $123.2(3)$ & $C(27 A)-C(26 A)-C(31 A)$ & $109.3(2)$ \\
\hline$C(15 A)-C(10 A)-C(11 A)$ & $115.6(2)$ & $C(27 A)-C(26 A)-P(1)$ & $112.95(18)$ \\
\hline$C(15 A)-C(10 A)-N(2 A)$ & $121.9(2)$ & $C(31 A)-C(26 A)-P(1)$ & $117.57(18)$ \\
\hline$C(11 \mathrm{~A})-\mathrm{C}(10 \mathrm{~A})-\mathrm{N}(2 \mathrm{~A})$ & $121.2(2)$ & $\mathrm{C}(26 \mathrm{~A})-\mathrm{C}(27 \mathrm{~A})-\mathrm{C}(28 \mathrm{~A})$ & $110.8(2)$ \\
\hline $\mathrm{F}(3 \mathrm{~A})-\mathrm{C}(11 \mathrm{~A})-\mathrm{C}(12 \mathrm{~A})$ & $119.3(2)$ & $C(29 A)-C(28 A)-C(27 A)$ & $111.5(2)$ \\
\hline $\mathrm{F}(3 \mathrm{~A})-\mathrm{C}(11 \mathrm{~A})-\mathrm{C}(10 \mathrm{~A})$ & $117.6(2)$ & $C(30 A)-C(29 A)-C(28 A)$ & $110.6(3)$ \\
\hline$C(12 A)-C(11 A)-C(10 A)$ & $123.0(3)$ & $C(31 A)-C(30 A)-C(29 A)$ & $111.2(2)$ \\
\hline$C(11 A)-C(12 A)-C(13 A)$ & $118.7(3)$ & $C(30 A)-C(31 A)-C(26 A)$ & $109.8(2)$ \\
\hline$C(14 A)-C(13 A)-C(12 A)$ & $121.3(3)$ & $C(37 A)-C(32 A)-C(33 A)$ & $112.4(2)$ \\
\hline$C(13 A)-C(14 A)-C(15 A)$ & $117.9(3)$ & $\mathrm{C}(37 \mathrm{~A})-\mathrm{C}(32 \mathrm{~A})-\mathrm{P}(1)$ & $114.55(18)$ \\
\hline $\mathrm{F}(4 \mathrm{~A})-\mathrm{C}(15 \mathrm{~A})-\mathrm{C}(10 \mathrm{~A})$ & $117.5(2)$ & $\mathrm{C}(33 \mathrm{~A})-\mathrm{C}(32 \mathrm{~A})-\mathrm{P}(1)$ & $113.59(18)$ \\
\hline $\mathrm{F}(4 \mathrm{~A})-\mathrm{C}(15 \mathrm{~A})-\mathrm{C}(14 \mathrm{~A})$ & $119.1(2)$ & $\mathrm{C}(34 \mathrm{~A})-\mathrm{C}(33 \mathrm{~A})-\mathrm{C}(32 \mathrm{~A})$ & $112.6(2)$ \\
\hline$C(10 A)-C(15 A)-C(14 A)$ & $123.4(3)$ & $\mathrm{C}(33 \mathrm{~A})-\mathrm{C}(34 \mathrm{~A})-\mathrm{C}(35 \mathrm{~A})$ & $112.6(2)$ \\
\hline$C(17 A)-C(16 A)-R u(1)$ & $127.9(2)$ & $C(34 A)-C(35 A)-C(36 A)$ & $109.4(2)$ \\
\hline $\mathrm{C}(18 \mathrm{~A})-\mathrm{C}(17 \mathrm{~A})-\mathrm{C}(22 \mathrm{~A})$ & $118.0(2)$ & $\mathrm{C}(35 \mathrm{~A})-\mathrm{C}(36 \mathrm{~A})-\mathrm{C}(37 \mathrm{~A})$ & $111.3(2)$ \\
\hline
\end{tabular}




\begin{tabular}{|c|c|c|c|}
\hline$C(36 A)-C(37 A)-C(32 A)$ & $112.1(2)$ & $\mathrm{C}(38 \mathrm{~B})-\mathrm{P}(2)-\mathrm{Ru}(2)$ & $111.63(8)$ \\
\hline $\mathrm{C}(43 \mathrm{~A})-\mathrm{C}(38 \mathrm{~A})-\mathrm{C}(39 \mathrm{~A})$ & $110.9(2)$ & $\mathrm{C}(22 \mathrm{~B})-\mathrm{O}(3 \mathrm{~B})-\mathrm{C}(23 \mathrm{~B})$ & $120.4(2)$ \\
\hline $\mathrm{C}(43 \mathrm{~A})-\mathrm{C}(38 \mathrm{~A})-\mathrm{P}(1)$ & $118.04(18)$ & $\mathrm{C}(1 \mathrm{~B})-\mathrm{N}(1 \mathrm{~B})-\mathrm{C}(4 \mathrm{~B})$ & $128.2(2)$ \\
\hline $\mathrm{C}(39 \mathrm{~A})-\mathrm{C}(38 \mathrm{~A})-\mathrm{P}(1)$ & $111.62(18)$ & $\mathrm{C}(1 \mathrm{~B})-\mathrm{N}(1 \mathrm{~B})-\mathrm{C}(2 \mathrm{~B})$ & $112.8(2)$ \\
\hline$C(38 A)-C(39 A)-C(40 A)$ & $112.4(2)$ & $\mathrm{C}(4 \mathrm{~B})-\mathrm{N}(1 \mathrm{~B})-\mathrm{C}(2 \mathrm{~B})$ & $117.4(2)$ \\
\hline$C(41 A)-C(40 A)-C(39 A)$ & $110.6(2)$ & $\mathrm{C}(1 \mathrm{~B})-\mathrm{N}(2 \mathrm{~B})-\mathrm{C}(10 \mathrm{~B})$ & $130.9(2)$ \\
\hline$C(42 A)-C(41 A)-C(40 A)$ & $110.3(2)$ & $\mathrm{C}(1 \mathrm{~B})-\mathrm{N}(2 \mathrm{~B})-\mathrm{C}(3 \mathrm{~B})$ & $113.8(2)$ \\
\hline$C(41 A)-C(42 A)-C(43 A)$ & $112.1(2)$ & $\mathrm{C}(10 \mathrm{~B})-\mathrm{N}(2 \mathrm{~B})-\mathrm{C}(3 \mathrm{~B})$ & $115.2(2)$ \\
\hline$C(38 A)-C(43 A)-C(42 A)$ & $111.3(2)$ & $\mathrm{O}(1 \mathrm{~B})-\mathrm{N}(3 \mathrm{~B})-\mathrm{O}(2 \mathrm{~B})$ & $123.1(2)$ \\
\hline$C(16 B)-R u(2)-C(1 B)$ & $92.57(10)$ & $\mathrm{O}(1 \mathrm{~B})-\mathrm{N}(3 \mathrm{~B})-\mathrm{C}(19 \mathrm{~B})$ & $118.7(2)$ \\
\hline$C(16 B)-R u(2)-P(2)$ & $88.70(8)$ & $\mathrm{O}(2 \mathrm{~B})-\mathrm{N}(3 \mathrm{~B})-\mathrm{C}(19 \mathrm{~B})$ & $118.3(2)$ \\
\hline $\mathrm{C}(1 \mathrm{~B})-\mathrm{Ru}(2)-\mathrm{P}(2)$ & $99.23(7)$ & N(2B)-C(1B)-N(1B) & $104.3(2)$ \\
\hline $\mathrm{C}(16 \mathrm{~B})-\mathrm{Ru}(2)-\mathrm{Cl}(2 \mathrm{~B})$ & $101.36(8)$ & $\mathrm{N}(2 \mathrm{~B})-\mathrm{C}(1 \mathrm{~B})-\mathrm{Ru}(2)$ & $130.02(19)$ \\
\hline $\mathrm{C}(1 \mathrm{~B})-\mathrm{Ru}(2)-\mathrm{Cl}(2 \mathrm{~B})$ & $165.02(7)$ & $\mathrm{N}(1 \mathrm{~B})-\mathrm{C}(1 \mathrm{~B})-\mathrm{Ru}(2)$ & $124.88(18)$ \\
\hline $\mathrm{P}(2)-\mathrm{Ru}(2)-\mathrm{Cl}(2 \mathrm{~B})$ & $86.67(2)$ & N(1B)-C(2B)-C(3B) & $100.34(19)$ \\
\hline $\mathrm{C}(16 \mathrm{~B})-\mathrm{Ru}(2)-\mathrm{Cl}(1 \mathrm{~B})$ & $99.02(8)$ & $\mathrm{N}(2 \mathrm{~B})-\mathrm{C}(3 \mathrm{~B})-\mathrm{C}(2 \mathrm{~B})$ & $100.9(2)$ \\
\hline $\mathrm{C}(1 \mathrm{~B})-\mathrm{Ru}(2)-\mathrm{Cl}(1 \mathrm{~B})$ & $84.60(7)$ & $C(5 B)-C(4 B)-C(9 B)$ & $115.4(2)$ \\
\hline $\mathrm{P}(2)-\mathrm{Ru}(2)-\mathrm{Cl}(1 \mathrm{~B})$ & $171.26(3)$ & $\mathrm{C}(5 \mathrm{~B})-\mathrm{C}(4 \mathrm{~B})-\mathrm{N}(1 \mathrm{~B})$ & $124.2(2)$ \\
\hline $\mathrm{Cl}(2 \mathrm{~B})-\mathrm{Ru}(2)-\mathrm{Cl}(1 \mathrm{~B})$ & $87.78(2)$ & C(9B)-C(4B)-N(1B) & $120.1(2)$ \\
\hline $\mathrm{F}(1 \mathrm{~B})-\mathrm{Ru}(2)-\mathrm{C}(1 \mathrm{~B})$ & $77.57(8)$ & $\mathrm{F}(1 \mathrm{~B})-\mathrm{C}(5 \mathrm{~B})-\mathrm{C}(4 \mathrm{~B})$ & $117.3(2)$ \\
\hline $\mathrm{F}(1 \mathrm{~B})-\mathrm{Ru}(2)-\mathrm{P}(2)$ & $102.54(4)$ & $\mathrm{F}(1 \mathrm{~B})-\mathrm{C}(5 \mathrm{~B})-\mathrm{C}(6 \mathrm{~B})$ & $119.1(2)$ \\
\hline $\mathrm{F}(1 \mathrm{~B})-\mathrm{Ru}(2)-\mathrm{C}(16 \mathrm{~B})$ & $166.01(8)$ & $C(4 B)-C(5 B)-C(6 B)$ & $123.6(2)$ \\
\hline $\mathrm{F}(1 \mathrm{~B})-\mathrm{Ru}(2)-\mathrm{Cl}(1 \mathrm{~B})$ & $70.47(4)$ & $C(7 B)-C(6 B)-C(5 B)$ & $118.7(3)$ \\
\hline $\mathrm{F}(1 \mathrm{~B})-\mathrm{Ru}(2)-\mathrm{Cl}(2 \mathrm{~B})$ & $87.74(4)$ & $\mathrm{C}(6 \mathrm{~B})-\mathrm{C}(7 \mathrm{~B})-\mathrm{C}(8 \mathrm{~B})$ & $120.2(3)$ \\
\hline $\mathrm{C}(26 \mathrm{~B})-\mathrm{P}(2)-\mathrm{C}(32 \mathrm{~B})$ & $102.98(12)$ & $\mathrm{C}(9 \mathrm{~B})-\mathrm{C}(8 \mathrm{~B})-\mathrm{C}(7 \mathrm{~B})$ & $118.9(3)$ \\
\hline $\mathrm{C}(26 \mathrm{~B})-\mathrm{P}(2)-\mathrm{C}(38 \mathrm{~B})$ & $101.62(12)$ & $\mathrm{F}(2 \mathrm{~B})-\mathrm{C}(9 \mathrm{~B})-\mathrm{C}(8 \mathrm{~B})$ & $118.4(2)$ \\
\hline $\mathrm{C}(32 \mathrm{~B})-\mathrm{P}(2)-\mathrm{C}(38 \mathrm{~B})$ & $104.52(12)$ & $\mathrm{F}(2 \mathrm{~B})-\mathrm{C}(9 \mathrm{~B})-\mathrm{C}(4 \mathrm{~B})$ & $118.5(2)$ \\
\hline$C(26 B)-P(2)-R u(2)$ & $120.41(9)$ & $C(8 B)-C(9 B)-C(4 B)$ & $123.1(2)$ \\
\hline $\mathrm{C}(32 \mathrm{~B})-\mathrm{P}(2)-\mathrm{Ru}(2)$ & $113.87(9)$ & $\mathrm{C}(15 \mathrm{~B})-\mathrm{C}(10 \mathrm{~B})-\mathrm{C}(11 \mathrm{~B})$ & $115.9(2)$ \\
\hline
\end{tabular}




\begin{tabular}{|c|c|c|c|}
\hline $\mathrm{C}(15 \mathrm{~B})-\mathrm{C}(10 \mathrm{~B})-\mathrm{N}(2 \mathrm{~B})$ & $122.4(2)$ & $\mathrm{C}(31 \mathrm{~B})-\mathrm{C}(26 \mathrm{~B})-\mathrm{P}(2)$ & $116.4(2)$ \\
\hline $\mathrm{C}(11 \mathrm{~B})-\mathrm{C}(10 \mathrm{~B})-\mathrm{N}(2 \mathrm{~B})$ & $120.2(2)$ & $\mathrm{C}(27 \mathrm{~B})-\mathrm{C}(26 \mathrm{~B})-\mathrm{P}(2)$ & $114.52(18)$ \\
\hline $\mathrm{F}(3 \mathrm{~B})-\mathrm{C}(11 \mathrm{~B})-\mathrm{C}(12 \mathrm{~B})$ & $118.8(2)$ & $\mathrm{C}(26 \mathrm{~B})-\mathrm{C}(27 \mathrm{~B})-\mathrm{C}(28 \mathrm{~B})$ & $110.7(2)$ \\
\hline $\mathrm{F}(3 \mathrm{~B})-\mathrm{C}(11 \mathrm{~B})-\mathrm{C}(10 \mathrm{~B})$ & $118.2(2)$ & $\mathrm{C}(29 \mathrm{~B})-\mathrm{C}(28 \mathrm{~B})-\mathrm{C}(27 \mathrm{~B})$ & $111.4(3)$ \\
\hline$C(12 B)-C(11 B)-C(10 B)$ & $122.9(3)$ & $\mathrm{C}(28 \mathrm{~B})-\mathrm{C}(29 \mathrm{~B})-\mathrm{C}(30 \mathrm{~B})$ & $112.0(2)$ \\
\hline$C(11 B)-C(12 B)-C(13 B)$ & 118.3(3) & $\mathrm{C}(29 \mathrm{~B})-\mathrm{C}(30 \mathrm{~B})-\mathrm{C}(31 \mathrm{~B})$ & $110.0(2)$ \\
\hline$C(14 B)-C(13 B)-C(12 B)$ & $121.2(3)$ & $\mathrm{C}(26 \mathrm{~B})-\mathrm{C}(31 \mathrm{~B})-\mathrm{C}(30 \mathrm{~B})$ & $110.0(2)$ \\
\hline$C(13 B)-C(14 B)-C(15 B)$ & $118.5(3)$ & $\mathrm{C}(33 \mathrm{~B})-\mathrm{C}(32 \mathrm{~B})-\mathrm{C}(37 \mathrm{~B})$ & $109.0(2)$ \\
\hline $\mathrm{F}(4 \mathrm{~B})-\mathrm{C}(15 \mathrm{~B})-\mathrm{C}(10 \mathrm{~B})$ & $117.8(2)$ & $\mathrm{C}(33 \mathrm{~B})-\mathrm{C}(32 \mathrm{~B})-\mathrm{P}(2)$ & $115.46(18)$ \\
\hline $\mathrm{F}(4 \mathrm{~B})-\mathrm{C}(15 \mathrm{~B})-\mathrm{C}(14 \mathrm{~B})$ & $119.0(2)$ & $\mathrm{C}(37 \mathrm{~B})-\mathrm{C}(32 \mathrm{~B})-\mathrm{P}(2)$ & 115.01(19) \\
\hline$C(10 B)-C(15 B)-C(14 B)$ & $123.1(3)$ & $\mathrm{C}(34 \mathrm{~B})-\mathrm{C}(33 \mathrm{~B})-\mathrm{C}(32 \mathrm{~B})$ & $111.8(2)$ \\
\hline$C(17 B)-C(16 B)-R u(2)$ & $128.3(2)$ & $\mathrm{C}(33 \mathrm{~B})-\mathrm{C}(34 \mathrm{~B})-\mathrm{C}(35 \mathrm{~B})$ & $112.5(2)$ \\
\hline $\mathrm{C}(18 \mathrm{~B})-\mathrm{C}(17 \mathrm{~B})-\mathrm{C}(22 \mathrm{~B})$ & $118.0(2)$ & $C(34 B)-C(35 B)-C(36 B)$ & $110.6(2)$ \\
\hline $\mathrm{C}(18 \mathrm{~B})-\mathrm{C}(17 \mathrm{~B})-\mathrm{C}(16 \mathrm{~B})$ & $122.4(2)$ & $C(35 B)-C(36 B)-C(37 B)$ & $111.4(3)$ \\
\hline$C(22 B)-C(17 B)-C(16 B)$ & $119.6(2)$ & $\mathrm{C}(36 \mathrm{~B})-\mathrm{C}(37 \mathrm{~B})-\mathrm{C}(32 \mathrm{~B})$ & $110.8(3)$ \\
\hline$C(19 B)-C(18 B)-C(17 B)$ & 119.2(2) & $\mathrm{C}(43 \mathrm{~B})-\mathrm{C}(38 \mathrm{~B})-\mathrm{C}(39 \mathrm{~B})$ & $109.3(2)$ \\
\hline$C(20 B)-C(19 B)-C(18 B)$ & $122.9(3)$ & $\mathrm{C}(43 \mathrm{~B})-\mathrm{C}(38 \mathrm{~B})-\mathrm{P}(2)$ & $116.87(18)$ \\
\hline $\mathrm{C}(20 \mathrm{~B})-\mathrm{C}(19 \mathrm{~B})-\mathrm{N}(3 \mathrm{~B})$ & $119.0(2)$ & $\mathrm{C}(39 \mathrm{~B})-\mathrm{C}(38 \mathrm{~B})-\mathrm{P}(2)$ & $112.97(17)$ \\
\hline $\mathrm{C}(18 \mathrm{~B})-\mathrm{C}(19 \mathrm{~B})-\mathrm{N}(3 \mathrm{~B})$ & $118.2(2)$ & $\mathrm{C}(40 \mathrm{~B})-\mathrm{C}(39 \mathrm{~B})-\mathrm{C}(38 \mathrm{~B})$ & $112.4(2)$ \\
\hline$C(21 B)-C(20 B)-C(19 B)$ & $118.6(3)$ & $\mathrm{C}(41 \mathrm{~B})-\mathrm{C}(40 \mathrm{~B})-\mathrm{C}(39 \mathrm{~B})$ & $112.5(2)$ \\
\hline$C(20 B)-C(21 B)-C(22 B)$ & $120.8(3)$ & $\mathrm{C}(40 \mathrm{~B})-\mathrm{C}(41 \mathrm{~B})-\mathrm{C}(42 \mathrm{~B})$ & $109.5(2)$ \\
\hline $\mathrm{O}(3 \mathrm{~B})-\mathrm{C}(22 \mathrm{~B})-\mathrm{C}(21 \mathrm{~B})$ & $123.8(2)$ & $\mathrm{C}(41 \mathrm{~B})-\mathrm{C}(42 \mathrm{~B})-\mathrm{C}(43 \mathrm{~B})$ & $110.4(2)$ \\
\hline $\mathrm{O}(3 \mathrm{~B})-\mathrm{C}(22 \mathrm{~B})-\mathrm{C}(17 \mathrm{~B})$ & $115.8(2)$ & $\mathrm{C}(38 \mathrm{~B})-\mathrm{C}(43 \mathrm{~B})-\mathrm{C}(42 \mathrm{~B})$ & $111.2(2)$ \\
\hline $\mathrm{C}(21 \mathrm{~B})-\mathrm{C}(22 \mathrm{~B})-\mathrm{C}(17 \mathrm{~B})$ & $120.5(2)$ & $\mathrm{Cl}(2)-\mathrm{C}(1)-\mathrm{Cl}(1)$ & $111.35(19)$ \\
\hline $\mathrm{O}(3 \mathrm{~B})-\mathrm{C}(23 \mathrm{~B})-\mathrm{C}(24 \mathrm{~B})$ & $105.1(2)$ & $\mathrm{Cl}(4)-\mathrm{C}(2)-\mathrm{Cl}(3)$ & $112.65(18)$ \\
\hline $\mathrm{O}(3 \mathrm{~B})-\mathrm{C}(23 \mathrm{~B})-\mathrm{C}(25 \mathrm{~B})$ & $109.9(2)$ & $\mathrm{Cl}(6)-\mathrm{C}(3)-\mathrm{Cl}(5)$ & $111.13(16)$ \\
\hline $\mathrm{C}(24 \mathrm{~B})-\mathrm{C}(23 \mathrm{~B})-\mathrm{C}(25 \mathrm{~B})$ & $112.3(2)$ & $\mathrm{Cl}(8)-\mathrm{C}(4)-\mathrm{Cl}(7)$ & $115.1(2)$ \\
\hline $\mathrm{C}(31 \mathrm{~B})-\mathrm{C}(26 \mathrm{~B})-\mathrm{C}(27 \mathrm{~B})$ & $108.9(2)$ & & \\
\hline
\end{tabular}


Table 5. Anisotropic displacement parameters $\left(\AA^{2} \mathbf{x} 10^{4}\right)$ for CCDC 284775 . The anisotropic displacement factor exponent takes the form: $-2 \pi^{2}\left[h^{2} a^{* 2} U 11+\ldots+2 h k\right.$ a* $b^{*}$ $\left.\mathbf{U}^{12}\right]$

\begin{tabular}{|c|c|c|c|c|c|c|}
\hline & $\mathrm{U}^{11}$ & $\mathrm{U}^{22}$ & $\mathrm{U}^{33}$ & $\mathrm{U}^{23}$ & $\mathrm{U}^{13}$ & $\mathrm{U}^{12}$ \\
\hline $\mathrm{Ru}(1)$ & $106(1)$ & $127(1)$ & $95(1)$ & $31(1)$ & $18(1)$ & $14(1)$ \\
\hline $\mathrm{Cl}(1 \mathrm{~A})$ & $118(3)$ & $173(4)$ & $185(4)$ & $68(3)$ & $47(3)$ & $17(3)$ \\
\hline $\mathrm{Cl}(2 \mathrm{~A})$ & 143(3) & 203(4) & $115(3)$ & $60(3)$ & $14(3)$ & $19(3)$ \\
\hline $\mathrm{P}(1)$ & $115(4)$ & $152(4)$ & $104(4)$ & $39(3)$ & $16(3)$ & $16(3)$ \\
\hline $\mathrm{F}(1 \mathrm{~A})$ & $225(9)$ & 243(9) & 172(9) & $3(7)$ & $-29(7)$ & $93(7)$ \\
\hline $\mathrm{F}(2 \mathrm{~A})$ & $292(10)$ & $269(10)$ & $237(10)$ & $80(8)$ & $-3(8)$ & $128(8)$ \\
\hline $\mathrm{F}(3 \mathrm{~A})$ & 183(9) & $222(9)$ & 244(9) & $18(7)$ & $77(7)$ & $-42(7)$ \\
\hline $\mathrm{F}(4 \mathrm{~A})$ & $169(9)$ & $288(10)$ & 187(9) & $4(7)$ & $-8(7)$ & $26(7)$ \\
\hline $\mathrm{O}(1 \mathrm{~A})$ & $275(12)$ & $164(12)$ & $445(14)$ & $33(10)$ & $-144(11)$ & $5(10)$ \\
\hline $\mathrm{O}(2 \mathrm{~A})$ & $253(12)$ & $177(11)$ & 381(13) & $73(10)$ & $-26(10)$ & $98(9)$ \\
\hline $\mathrm{O}(3 \mathrm{~A})$ & $161(11)$ & $148(10)$ & $198(11)$ & $1(8)$ & $-36(9)$ & $-3(8)$ \\
\hline $\mathrm{N}(1 \mathrm{~A})$ & $200(13)$ & $97(12)$ & $97(12)$ & $24(9)$ & $23(10)$ & $23(10)$ \\
\hline $\mathrm{N}(2 \mathrm{~A})$ & $170(13)$ & $124(12)$ & $97(12)$ & $32(9)$ & $20(10)$ & $32(10)$ \\
\hline $\mathrm{N}(3 \mathrm{~A})$ & 191(14) & $199(14)$ & $255(15)$ & $100(12)$ & $36(11)$ & $9(11)$ \\
\hline$C(1 \mathrm{~A})$ & $53(13)$ & $163(15)$ & $159(15)$ & $58(12)$ & $7(11)$ & $7(11)$ \\
\hline$C(2 \mathrm{~A})$ & $307(18)$ & $178(16)$ & $130(15)$ & $77(12)$ & $37(13)$ & $54(13)$ \\
\hline$C(3 \mathrm{~A})$ & $252(16)$ & $162(15)$ & $121(15)$ & $56(12)$ & $25(12)$ & $20(13)$ \\
\hline $\mathrm{C}(4 \mathrm{~A})$ & $156(15)$ & $118(14)$ & $150(15)$ & $49(12)$ & $54(12)$ & $-16(11)$ \\
\hline$C(5 \mathrm{~A})$ & $125(15)$ & $163(15)$ & $185(16)$ & $44(12)$ & $48(12)$ & $16(12)$ \\
\hline$C(6 A)$ & $166(16)$ & $232(17)$ & $152(16)$ & $29(13)$ & $29(12)$ & $-52(13)$ \\
\hline$C(7 A)$ & $254(17)$ & $163(16)$ & $223(17)$ & $-11(13)$ & $125(14)$ & $-37(13)$ \\
\hline $\mathrm{C}(8 \mathrm{~A})$ & $271(18)$ & $138(16)$ & $267(18)$ & $63(13)$ & $124(14)$ & $66(13)$ \\
\hline$C(9 A)$ & $192(16)$ & $203(16)$ & $169(16)$ & $56(13)$ & $42(13)$ & $13(13)$ \\
\hline$C(10 A)$ & $212(16)$ & $149(15)$ & $75(14)$ & $35(11)$ & $33(12)$ & $33(12)$ \\
\hline
\end{tabular}




\begin{tabular}{|c|c|c|c|c|c|c|}
\hline$C(11 A)$ & 194(16) & $172(16)$ & $112(15)$ & $25(12)$ & $45(12)$ & $-30(12)$ \\
\hline$C(12 A)$ & $214(17)$ & $214(17)$ & 206(16) & $53(13)$ & $76(13)$ & $70(13)$ \\
\hline$C(13 A)$ & $360(20)$ & $189(17)$ & 191(17) & $46(13)$ & $90(14)$ & $80(14)$ \\
\hline$C(14 A)$ & $265(18)$ & 171(16) & $163(16)$ & $-1(13)$ & $-13(13)$ & $-36(13)$ \\
\hline$C(15 A)$ & 179(16) & $262(17)$ & $96(15)$ & $75(12)$ & $23(12)$ & $23(13)$ \\
\hline$C(16 A)$ & $119(14)$ & $173(15)$ & $98(14)$ & $47(11)$ & $39(11)$ & $-7(11)$ \\
\hline$C(17 A)$ & $134(14)$ & $124(14)$ & $117(14)$ & $23(11)$ & $53(11)$ & $2(11)$ \\
\hline $\mathrm{C}(18 \mathrm{~A})$ & $153(15)$ & $144(15)$ & $146(15)$ & $40(12)$ & $49(12)$ & $-21(12)$ \\
\hline$C(19 A)$ & $152(15)$ & $157(15)$ & $167(15)$ & $47(12)$ & $42(12)$ & $27(12)$ \\
\hline$C(20 A)$ & $200(16)$ & $142(15)$ & $264(17)$ & $52(13)$ & $90(13)$ & $67(13)$ \\
\hline$C(21 A)$ & 199(16) & $130(15)$ & 198(16) & $-16(12)$ & $31(13)$ & $15(12)$ \\
\hline$C(22 A)$ & $128(15)$ & $221(16)$ & $136(15)$ & $35(12)$ & $50(12)$ & $9(12)$ \\
\hline$C(23 A)$ & $220(17)$ & 161(16) & 273(18) & $-21(13)$ & $-24(14)$ & $-6(13)$ \\
\hline$C(24 A)$ & $318(19)$ & $270(18)$ & $303(19)$ & $30(15)$ & $-133(15)$ & $-102(15)$ \\
\hline$C(25 A)$ & $263(18)$ & $249(18)$ & $380(20)$ & $70(15)$ & $-2(15)$ & $-52(14)$ \\
\hline$C(26 A)$ & $136(15)$ & $188(16)$ & $157(15)$ & $43(12)$ & $0(12)$ & $37(12)$ \\
\hline$C(27 A)$ & $157(15)$ & $314(18)$ & $148(16)$ & $78(13)$ & $15(12)$ & $51(13)$ \\
\hline$C(28 \mathrm{~A})$ & $246(18)$ & $400(20)$ & $265(18)$ & 182(16) & $0(14)$ & $50(15)$ \\
\hline$C(29 A)$ & $228(18)$ & $540(20)$ & 243(19) & $92(17)$ & $-74(14)$ & $-2(16)$ \\
\hline$C(30 A)$ & $214(17)$ & $370(20)$ & $244(18)$ & $13(15)$ & $-52(14)$ & $-8(15)$ \\
\hline$C(31 \mathrm{~A})$ & 192(16) & 292(18) & 181(16) & $20(13)$ & $-34(13)$ & $7(13)$ \\
\hline$C(32 \mathrm{~A})$ & $147(15)$ & $208(16)$ & $122(15)$ & $73(12)$ & $39(12)$ & $-2(12)$ \\
\hline$C(33 A)$ & $178(16)$ & $176(16)$ & 197(16) & $58(12)$ & $54(13)$ & $32(12)$ \\
\hline$C(34 \mathrm{~A})$ & $226(17)$ & 199(16) & $212(16)$ & $61(13)$ & $60(13)$ & $-3(13)$ \\
\hline$C(35 A)$ & $232(17)$ & $236(17)$ & $220(17)$ & $90(14)$ & $32(13)$ & $-60(13)$ \\
\hline$C(36 A)$ & $186(16)$ & $279(18)$ & $236(17)$ & $109(14)$ & $81(13)$ & $-11(13)$ \\
\hline$C(37 A)$ & $172(15)$ & $220(16)$ & $209(16)$ & $73(13)$ & $52(13)$ & $48(13)$ \\
\hline$C(38 \mathrm{~A})$ & $117(14)$ & $159(15)$ & $140(15)$ & $33(12)$ & $25(11)$ & $15(11)$ \\
\hline$C(39 A)$ & $157(15)$ & $274(17)$ & $132(15)$ & $24(13)$ & $-8(12)$ & $78(13)$ \\
\hline
\end{tabular}




\begin{tabular}{|c|c|c|c|c|c|c|}
\hline$C(40 A)$ & $176(16)$ & $240(17)$ & 141(15) & $-4(13)$ & $-4(12)$ & $3(13)$ \\
\hline$C(41 \mathrm{~A})$ & 213(16) & $178(16)$ & $156(15)$ & $14(12)$ & $34(13)$ & $21(13)$ \\
\hline $\mathrm{C}(42 \mathrm{~A})$ & $145(15)$ & $176(15)$ & $192(16)$ & $52(12)$ & $68(12)$ & $54(12)$ \\
\hline$C(43 A)$ & $140(15)$ & $220(16)$ & $119(15)$ & $56(12)$ & $38(12)$ & $19(12)$ \\
\hline $\mathrm{Ru}(2)$ & $95(1)$ & $128(1)$ & $91(1)$ & $26(1)$ & $15(1)$ & $7(1)$ \\
\hline $\mathrm{Cl}(1 \mathrm{~B})$ & $99(3)$ & $184(4)$ & $167(4)$ & $53(3)$ & $25(3)$ & $15(3)$ \\
\hline $\mathrm{Cl}(2 \mathrm{~B})$ & $144(3)$ & $226(4)$ & 111(3) & $62(3)$ & $11(3)$ & $17(3)$ \\
\hline $\mathrm{P}(2)$ & $96(4)$ & $152(4)$ & 101(4) & $37(3)$ & $15(3)$ & $6(3)$ \\
\hline $\mathrm{F}(1 \mathrm{~B})$ & 205(9) & $214(9)$ & 153(9) & $24(7)$ & $-7(7)$ & $60(7)$ \\
\hline $\mathrm{F}(2 \mathrm{~B})$ & $216(9)$ & 197(9) & 166(9) & $55(7)$ & $2(7)$ & $45(7)$ \\
\hline $\mathrm{F}(3 \mathrm{~B})$ & 181(9) & 221(9) & 234(9) & $-2(7)$ & $56(7)$ & $-48(7)$ \\
\hline $\mathrm{F}(4 \mathrm{~B})$ & 170(9) & $300(10)$ & 207(9) & $-4(8)$ & $6(7)$ & $24(7)$ \\
\hline $\mathrm{O}(1 \mathrm{~B})$ & $338(13)$ & $272(13)$ & $324(14)$ & $11(11)$ & $-116(11)$ & $50(10)$ \\
\hline $\mathrm{O}(2 \mathrm{~B})$ & $319(13)$ & $184(12)$ & $473(15)$ & $95(11)$ & $-69(11)$ & $98(10)$ \\
\hline $\mathrm{O}(3 \mathrm{~B})$ & 193(11) & $133(10)$ & $196(11)$ & $15(8)$ & $-23(9)$ & $-14(8)$ \\
\hline $\mathrm{N}(1 \mathrm{~B})$ & $153(12)$ & $110(12)$ & $78(12)$ & $2(9)$ & $51(9)$ & $13(9)$ \\
\hline $\mathrm{N}(2 \mathrm{~B})$ & $168(13)$ & $113(12)$ & $113(12)$ & $36(9)$ & $43(10)$ & $20(10)$ \\
\hline $\mathrm{N}(3 \mathrm{~B})$ & $245(15)$ & $179(14)$ & $284(16)$ & $83(12)$ & $12(12)$ & $11(12)$ \\
\hline $\mathrm{C}(1 \mathrm{~B})$ & $61(13)$ & $130(14)$ & $122(14)$ & $1(11)$ & $-15(11)$ & $-4(11)$ \\
\hline $\mathrm{C}(2 \mathrm{~B})$ & $173(15)$ & $170(15)$ & $142(15)$ & $49(12)$ & $46(12)$ & $-7(12)$ \\
\hline $\mathrm{C}(3 \mathrm{~B})$ & $246(16)$ & $155(15)$ & $140(15)$ & $69(12)$ & $62(12)$ & $38(12)$ \\
\hline$C(4 B)$ & $122(14)$ & $120(14)$ & $133(15)$ & $25(11)$ & $55(11)$ & $-5(11)$ \\
\hline$C(5 B)$ & $102(14)$ & 107(14) & $185(16)$ & $43(12)$ & $45(12)$ & $11(11)$ \\
\hline$C(6 B)$ & $148(15)$ & $206(16)$ & $109(15)$ & $16(12)$ & $48(12)$ & $-42(12)$ \\
\hline$C(7 B)$ & 194(16) & $108(15)$ & $210(16)$ & $-13(12)$ & $84(13)$ & $0(12)$ \\
\hline $\mathrm{C}(8 \mathrm{~B})$ & $159(15)$ & $157(15)$ & $209(16)$ & $62(13)$ & $50(12)$ & $24(12)$ \\
\hline $\mathrm{C}(9 \mathrm{~B})$ & $129(14)$ & $155(15)$ & $134(15)$ & $35(12)$ & $29(12)$ & $-2(12)$ \\
\hline$C(10 B)$ & $188(15)$ & $95(14)$ & 104(14) & $21(11)$ & $60(12)$ & $22(11)$ \\
\hline
\end{tabular}




\begin{tabular}{|c|c|c|c|c|c|c|}
\hline$C(11 B)$ & 181(16) & $165(15)$ & $140(15)$ & $38(12)$ & $41(12)$ & $-41(12)$ \\
\hline$C(12 B)$ & $200(16)$ & $215(17)$ & $258(17)$ & $85(14)$ & $98(14)$ & $83(13)$ \\
\hline$C(13 B)$ & 297(18) & $136(16)$ & 264(18) & $15(13)$ & $125(14)$ & $45(13)$ \\
\hline$C(14 B)$ & $254(18)$ & $177(16)$ & $195(16)$ & $-5(13)$ & $36(13)$ & $-24(13)$ \\
\hline$C(15 B)$ & $141(15)$ & $233(17)$ & $175(16)$ & $73(13)$ & $56(12)$ & $39(13)$ \\
\hline$C(16 B)$ & $95(14)$ & $156(15)$ & $121(14)$ & $48(11)$ & $28(11)$ & $-2(11)$ \\
\hline$C(17 B)$ & $127(14)$ & $170(15)$ & $134(15)$ & $49(12)$ & $79(12)$ & $29(12)$ \\
\hline $\mathrm{C}(18 \mathrm{~B})$ & $141(15)$ & 141(15) & $147(15)$ & $21(12)$ & $38(12)$ & $4(12)$ \\
\hline$C(19 B)$ & $164(15)$ & $177(16)$ & $179(16)$ & $63(12)$ & $-10(12)$ & $37(12)$ \\
\hline$C(20 B)$ & $205(16)$ & 141(15) & 243(17) & $72(13)$ & $57(13)$ & $42(12)$ \\
\hline$C(21 B)$ & 213(16) & $117(15)$ & $215(16)$ & $20(12)$ & $41(13)$ & $-13(12)$ \\
\hline$C(22 B)$ & $123(14)$ & $165(15)$ & $133(15)$ & $32(12)$ & $24(12)$ & $0(12)$ \\
\hline$C(23 B)$ & $188(16)$ & 151(16) & $237(17)$ & $-5(13)$ & $-7(13)$ & $-31(12)$ \\
\hline$C(24 B)$ & $267(18)$ & $267(18)$ & $253(18)$ & $38(14)$ & $-54(14)$ & $-31(14)$ \\
\hline$C(25 B)$ & $230(17)$ & 281(18) & $337(19)$ & $102(15)$ & $12(15)$ & $-49(14)$ \\
\hline$C(26 B)$ & $98(14)$ & $266(17)$ & $158(15)$ & $81(13)$ & $-24(12)$ & $-24(12)$ \\
\hline$C(27 B)$ & 191(16) & $259(17)$ & $158(16)$ & $82(13)$ & $-6(12)$ & $-38(13)$ \\
\hline $\mathrm{C}(28 \mathrm{~B})$ & $309(19)$ & $350(20)$ & 279(19) & $169(16)$ & $-23(15)$ & $7(15)$ \\
\hline$C(29 B)$ & $410(20)$ & $304(19)$ & 209(18) & $71(15)$ & $-112(15)$ & $-6(16)$ \\
\hline $\mathrm{C}(30 \mathrm{~B})$ & $360(20)$ & $258(18)$ & $255(18)$ & $63(15)$ & $-126(15)$ & $-47(15)$ \\
\hline$C(31 B)$ & $289(18)$ & $321(19)$ & $226(18)$ & $83(15)$ & $-3(14)$ & $36(15)$ \\
\hline$C(32 B)$ & $156(15)$ & $187(15)$ & $107(14)$ & $40(12)$ & $24(11)$ & $-31(12)$ \\
\hline$C(33 B)$ & $245(17)$ & 193(16) & $262(18)$ & $92(13)$ & $119(14)$ & $42(13)$ \\
\hline$C(34 B)$ & 271(18) & $277(18)$ & $336(19)$ & $162(15)$ & $55(15)$ & $18(14)$ \\
\hline$C(35 B)$ & $267(18)$ & $274(18)$ & 274(18) & $137(15)$ & $72(14)$ & $-51(14)$ \\
\hline$C(36 B)$ & $380(20)$ & $330(20)$ & $540(20)$ & $170(18)$ & 295(19) & $13(16)$ \\
\hline$C(37 B)$ & 261(18) & $246(18)$ & $510(20)$ & $157(16)$ & 190(16) & $40(14)$ \\
\hline $\mathrm{C}(38 \mathrm{~B})$ & $126(14)$ & $149(15)$ & $134(15)$ & $50(12)$ & $26(11)$ & $37(11)$ \\
\hline$C(39 B)$ & $179(15)$ & 195(16) & $160(15)$ & $42(12)$ & $5(12)$ & $52(12)$ \\
\hline
\end{tabular}




\begin{tabular}{|c|c|c|c|c|c|c|}
\hline $\mathrm{C}(40 \mathrm{~B})$ & $180(15)$ & $206(16)$ & $109(15)$ & $34(12)$ & $20(12)$ & $32(12)$ \\
\hline $\mathrm{C}(41 \mathrm{~B})$ & $205(16)$ & $232(17)$ & $170(16)$ & $33(13)$ & $44(13)$ & $70(13)$ \\
\hline $\mathrm{C}(42 \mathrm{~B})$ & $167(16)$ & $266(17)$ & $190(16)$ & $24(13)$ & $45(13)$ & $84(13)$ \\
\hline$C(43 B)$ & $115(15)$ & $223(16)$ & 189(16) & $27(13)$ & $9(12)$ & $27(12)$ \\
\hline $\mathrm{C}(1)$ & $460(20)$ & $510(30)$ & $580(30)$ & $30(20)$ & $30(20)$ & $250(20)$ \\
\hline $\mathrm{Cl}(1)$ & $856(8)$ & $499(6)$ & $452(6)$ & $116(5)$ & $308(6)$ & $57(6)$ \\
\hline $\mathrm{Cl}(2)$ & $614(7)$ & $705(7)$ & $494(6)$ & $254(5)$ & $112(5)$ & $320(6)$ \\
\hline$C(2)$ & $268(19)$ & $520(20)$ & $310(20)$ & $155(17)$ & $12(15)$ & $-68(16)$ \\
\hline $\mathrm{Cl}(3)$ & $265(4)$ & $372(5)$ & $474(5)$ & $112(4)$ & $166(4)$ & $61(4)$ \\
\hline $\mathrm{Cl}(4)$ & $379(5)$ & $845(8)$ & $400(6)$ & $196(5)$ & $-82(4)$ & $-285(5)$ \\
\hline$C(3)$ & 287(19) & $225(18)$ & $400(20)$ & $44(15)$ & $-20(16)$ & $63(14)$ \\
\hline $\mathrm{Cl}(5)$ & $275(5)$ & $425(5)$ & $546(6)$ & $183(5)$ & $-53(4)$ & $61(4)$ \\
\hline $\mathrm{Cl}(6)$ & 752(7) & $281(5)$ & $424(6)$ & $55(4)$ & $-74(5)$ & $14(5)$ \\
\hline C(4) & $330(20)$ & $950(40)$ & $1150(40)$ & $720(30)$ & $-20(30)$ & $110(20)$ \\
\hline $\mathrm{Cl}(7)$ & $442(6)$ & $570(6)$ & $478(6)$ & $237(5)$ & $174(5)$ & $85(5)$ \\
\hline $\mathrm{Cl}(8)$ & $605(8)$ & 1040(11) & $1416(13)$ & $106(10)$ & $525(9)$ & $6(7)$ \\
\hline
\end{tabular}


Bis(F4-NHC)-derived ruthenium complex S8

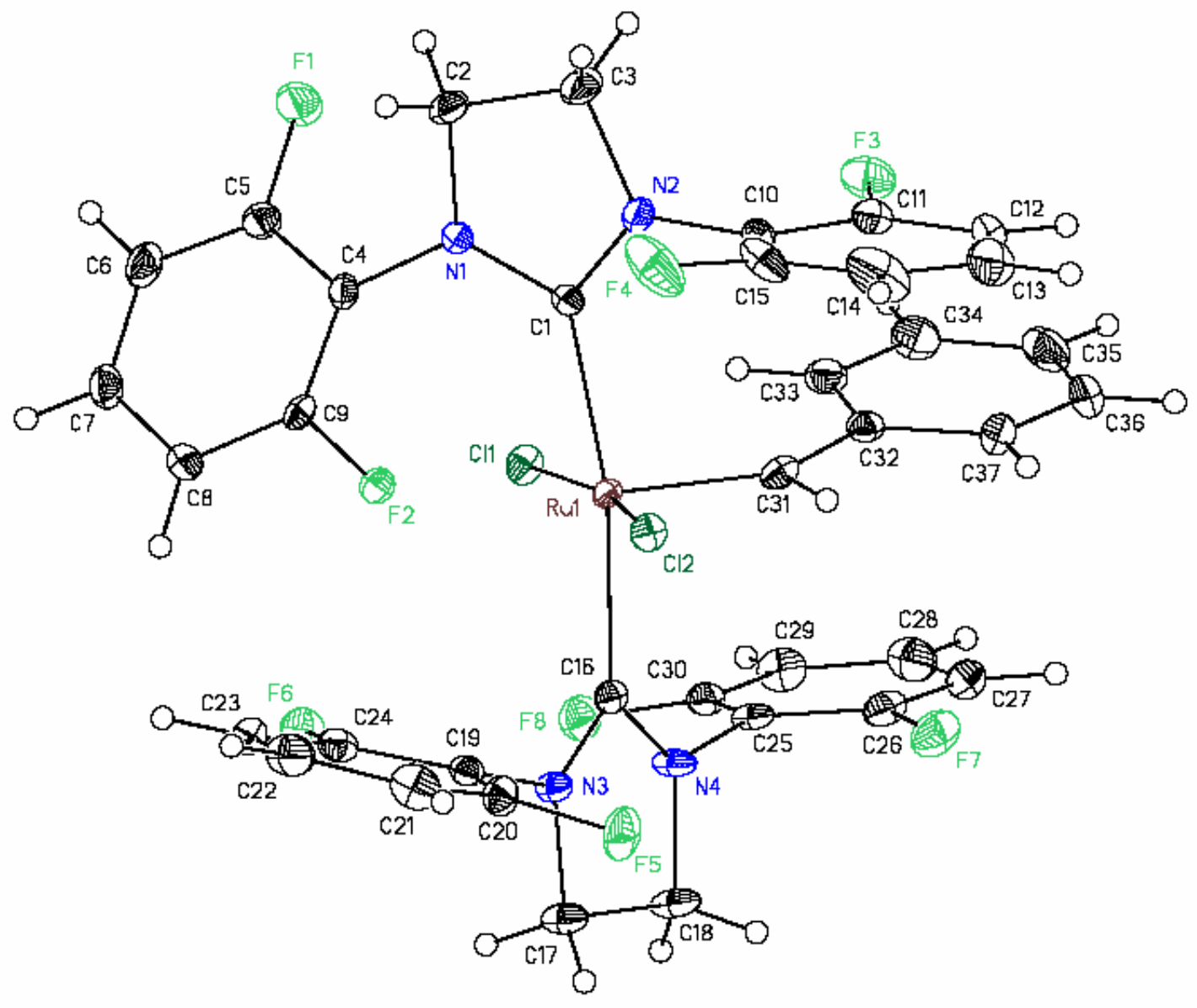


Table 1. Crystal data and structure refinement for CCDC 279135.

Empirical formula

Formula weight

Crystallization Solvent

Crystal Habit

Crystal size

Crystal color

ype of diffractometer

Wavelength

Data Collection Temperature

$\theta$ range for 16225 reflections used

in lattice determination

Unit cell dimensions

Volume

Z

Crystal system

Space group

Density (calculated)

$\mathrm{F}(000)$

Data collection program

$\theta$ range for data collection

Completeness to $\theta=40.73^{\circ}$
$\mathrm{C}_{37} \mathrm{H}_{26} \mathrm{~F}_{8} \mathrm{~N}_{4} \mathrm{Cl}_{2} \mathrm{Ru} \cdot 1 /{ }_{4}\left(\mathrm{C}_{4} \mathrm{H}_{10} \mathrm{O}\right) \cdot \mathrm{C}_{4} \mathrm{H}_{8} \mathrm{O}$

941.22

THF/diethylether

Block

$0.22 \times 0.19 \times 0.19 \mathrm{~mm}^{3}$

Dichroic - red/green

\section{Data Collection}

Bruker SMART 1000

$0.71073 \AA \mathrm{MoK} \alpha$

100(2) K

2.37 to $38.62^{\circ}$

$\mathrm{a}=27.2151(8) \AA$

$c=10.5174(4) \AA$

7789.8(4) $\AA^{3}$

8

Tetragonal

I-4

$1.605 \mathrm{Mg} / \mathrm{m}^{3}$

3812

Bruker SMART v5.630

2.08 to $40.73^{\circ}$

$88.2 \%$ 
Index ranges

Data collection scan type

Data reduction program

Reflections collected

Independent reflections

Absorption coefficient

Absorption correction

Max. and min. transmission
$-34 \leq \mathrm{h} \leq 40,-48 \leq \mathrm{k} \leq 49,-18 \leq 1 \leq 15$

$\omega$ scans at $3 \phi$ settings

Bruker SAINT v6.45A

45166

$19977\left[\mathrm{R}_{\mathrm{int}}=0.0759\right]$

$0.620 \mathrm{~mm}^{-1}$

None

0.8914 and 0.8757 


\section{Table 1 (cont.)}

\section{Structure solution and Refinement}

\begin{tabular}{|c|c|}
\hline Structure solution program & Bruker XS v6.12 \\
\hline Primary solution method & Direct methods \\
\hline Secondary solution method & Difference Fourier map \\
\hline Hydrogen placement & Geometric positions \\
\hline Structure refinement program & Bruker XL v6.12 \\
\hline Refinement method & Full matrix least-squares on $\mathrm{F}^{2}$ \\
\hline Data / restraints / parameters & 19977 / 0 / 534 \\
\hline Treatment of hydrogen atoms & Riding \\
\hline Goodness-of-fit on $\mathrm{F}^{2}$ & 0.946 \\
\hline Final $R$ indices $[I>2 \sigma(I), 13960$ reflections $]$ & $\mathrm{R} 1=0.0441, w \mathrm{R} 2=0.0685$ \\
\hline $\mathrm{R}$ indices (all data) & $\mathrm{R} 1=0.0771, w \mathrm{R} 2=0.0748$ \\
\hline Type of weighting scheme used & Sigma \\
\hline Weighting scheme used & $w=1 / \sigma^{2}\left(\mathrm{Fo}^{2}\right)$ \\
\hline Max shift/error & 0.004 \\
\hline Average shift/error & 0.003 \\
\hline Absolute structure determination & Unknown \\
\hline Absolute structure parameter & 0.00 \\
\hline Largest diff. peak and hole & 1.528 and -0.895 e. $\AA^{-3}$ \\
\hline
\end{tabular}

\section{Special Refinement Details}

The 2,6-difluorophenyl ring containing atoms labeled $\mathrm{C}(4)-\mathrm{C}(9)$ tilts by nearly $60^{\circ}$ so as to place one halogen near the metal center $(\operatorname{Ru}(1)-\mathrm{F}(2)=2.5203(12) \AA$, see Table 6 and Figure 2). As shown in the table, the torsion angles for the other three rings tend toward $90^{\circ}$. Another feature that contributes to this conformation is a rotation of the imidazole ligands by approximately $40^{\circ}$ around the bond to the metal, with respect to the metal- 
benzylidene bond (see Table 6 and Figure 3).

The crystals contain solvent of crystallization. Each asymmetric unit contains one molecule of THF and one-fourth of a disordered solvent best modeled as diethylether at a 4-bar special position.

Refinement of $\mathrm{F}^{2}$ against ALL reflections. The weighted R-factor $(w \mathrm{R})$ and goodness of fit $(\mathrm{S})$ are based on $\mathrm{F}^{2}$, conventional R-factors (R) are based on $\mathrm{F}$, with $\mathrm{F}$ set to zero for negative $\mathrm{F}^{2}$. The threshold expression of $\mathrm{F}^{2}$ $>2 \sigma\left(\mathrm{F}^{2}\right)$ is used only for calculating R-factors(gt) etc. and is not relevant to the choice of reflections for refinement. R-factors based on $\mathrm{F}^{2}$ are statistically about twice as large as those based on $\mathrm{F}$, and R-factors based on ALL data will be even larger.

All esds (except the esd in the dihedral angle between two 1.s. planes) are estimated using the full covariance matrix. The cell esds are taken into account individually in the estimation of esds in distances, angles and torsion angles; correlations between esds in cell parameters are only used when they are defined by crystal symmetry. An approximate (isotropic) treatment of cell esds is used for estimating esds involving l.s. planes. 


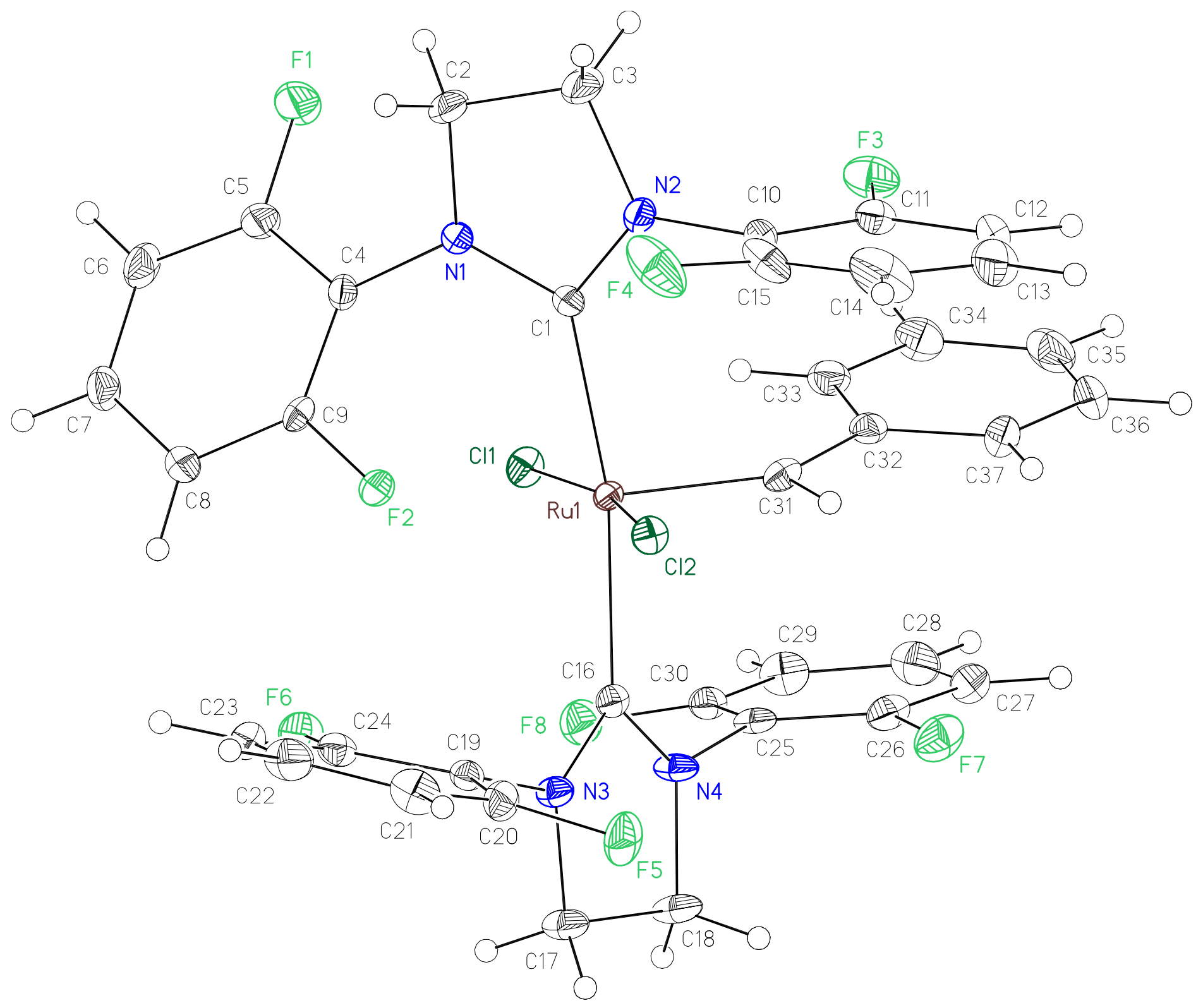




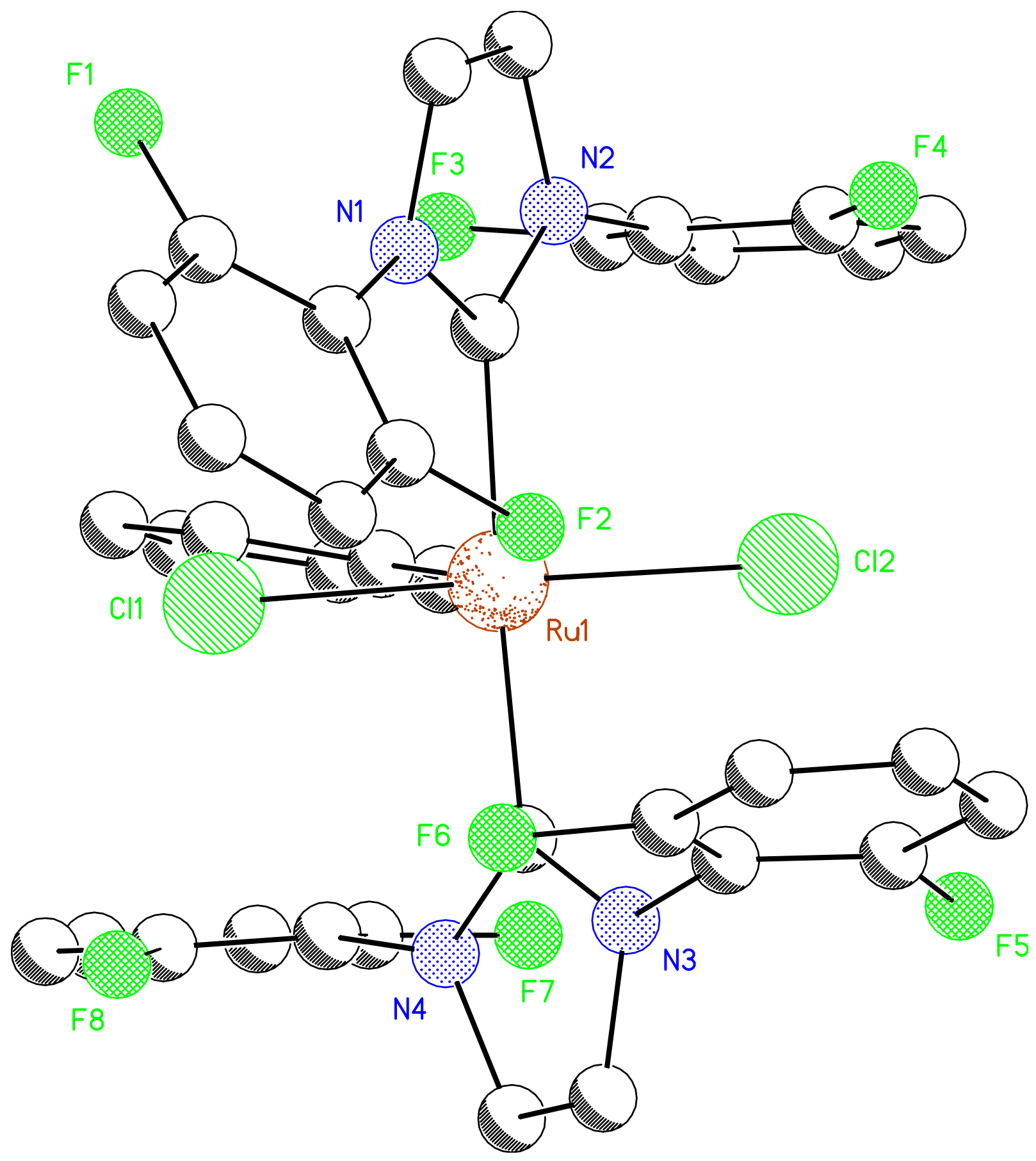




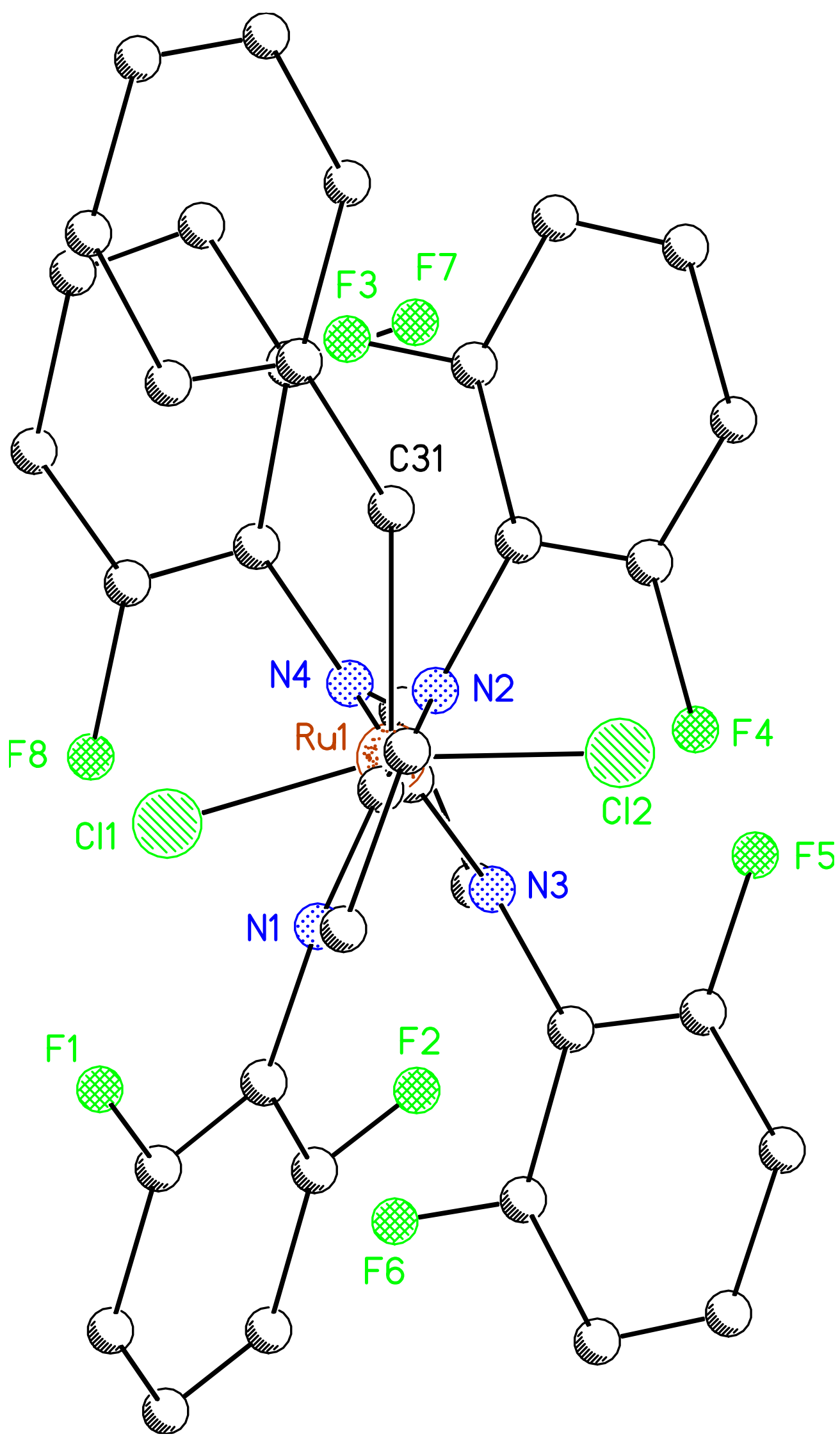



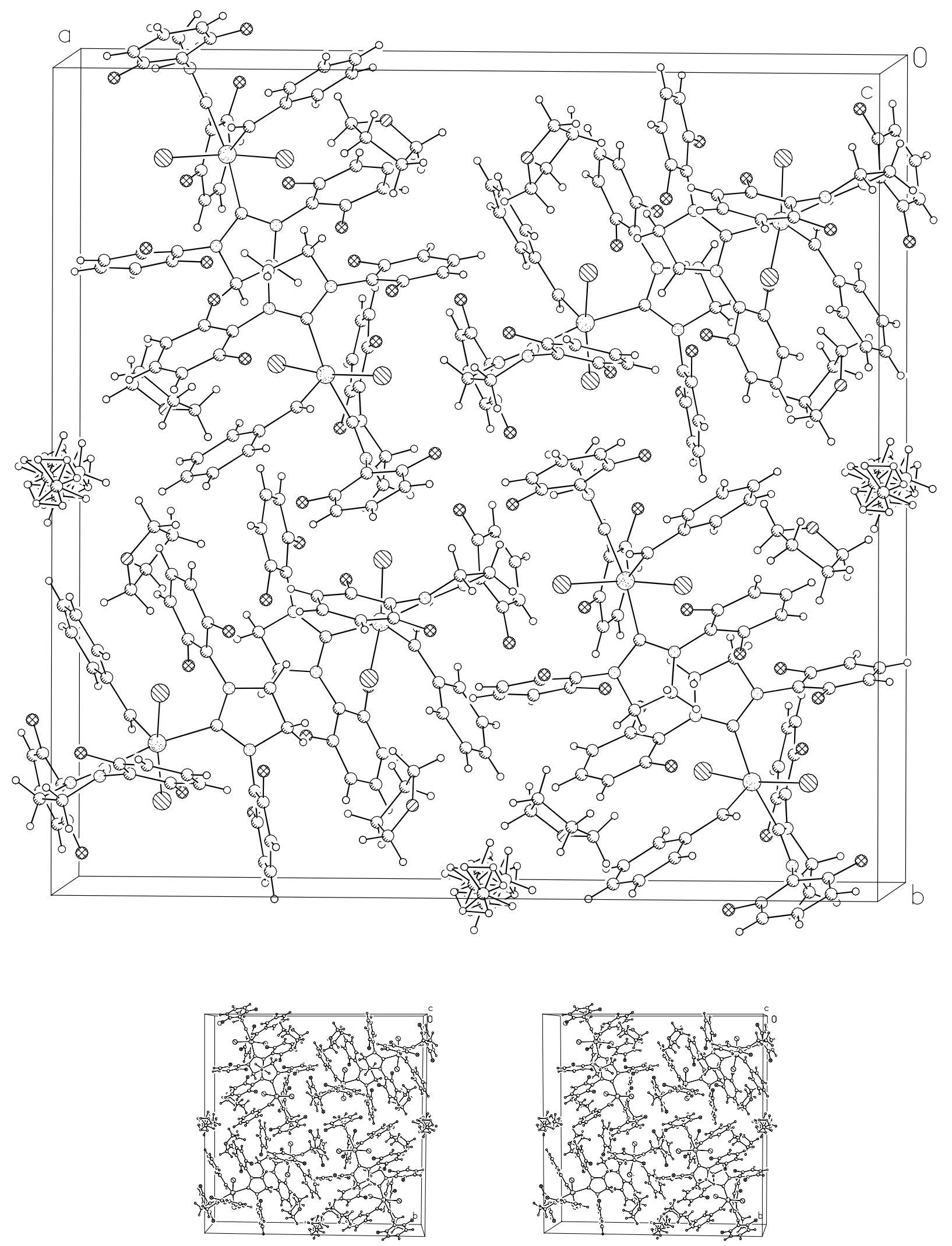
Table 2. Atomic coordinates $\left(\mathrm{x} \mathrm{10}^{4}\right)$ and equivalent isotropic displacement parameters $\left(\AA^{2} \times 1^{3}\right)$ for CCDC 279135 . U(eq) is defined as the trace of the orthogonalized $U^{i j}$ tensor.

\begin{tabular}{|c|c|c|c|c|}
\hline & $\mathrm{x}$ & $\mathrm{y}$ & $\mathrm{z}$ & $\mathrm{U}_{\text {eq }}$ \\
\hline $\mathrm{Ru}(1)$ & $8183(1)$ & $1233(1)$ & $1329(1)$ & $10(1)$ \\
\hline $\mathrm{Cl}(1)$ & $7540(1)$ & 1321(1) & $-219(1)$ & $15(1)$ \\
\hline $\mathrm{Cl}(2)$ & $8910(1)$ & $1253(1)$ & $2617(1)$ & $14(1)$ \\
\hline $\mathrm{F}(1)$ & $8197(1)$ & $473(1)$ & $-3464(1)$ & $29(1)$ \\
\hline $\mathrm{F}(2)$ & $8733(1)$ & $1597(1)$ & $-354(1)$ & $15(1)$ \\
\hline $\mathrm{F}(3)$ & $7928(1)$ & $-299(1)$ & 2031(1) & $25(1)$ \\
\hline $\mathrm{F}(4)$ & 9531(1) & $305(1)$ & 1994(2) & $37(1)$ \\
\hline$F(5)$ & $9073(1)$ & $2313(1)$ & $3978(1)$ & $24(1)$ \\
\hline$F(6)$ & $8476(1)$ & $2573(1)$ & $-141(1)$ & $22(1)$ \\
\hline $\mathrm{F}(7)$ & $7324(1)$ & $1496(1)$ & $4765(1)$ & $25(1)$ \\
\hline $\mathrm{F}(8)$ & 6814(1) & 2116(1) & $824(1)$ & $22(1)$ \\
\hline $\mathrm{N}(1)$ & $8533(1)$ & $621(1)$ & $-991(2)$ & $15(1)$ \\
\hline $\mathrm{N}(2)$ & $8655(1)$ & $208(1)$ & $738(2)$ & $18(1)$ \\
\hline $\mathrm{N}(3)$ & $8279(1)$ & 2321(1) & $2337(2)$ & $13(1)$ \\
\hline $\mathrm{N}(4)$ & 7544(1) & $2058(1)$ & $2647(2)$ & $15(1)$ \\
\hline$C(1)$ & $8474(1)$ & $635(1)$ & $303(2)$ & $13(1)$ \\
\hline$C(2)$ & $8817(1)$ & 181(1) & $-1424(2)$ & $24(1)$ \\
\hline$C(3)$ & $8827(1)$ & $-133(1)$ & $-256(2)$ & $35(1)$ \\
\hline$C(4)$ & $8481(1)$ & $1012(1)$ & $-1857(2)$ & $13(1)$ \\
\hline$C(5)$ & $8335(1)$ & $929(1)$ & $-3116(2)$ & $18(1)$ \\
\hline$C(6)$ & $8325(1)$ & 1294(1) & $-4026(2)$ & $20(1)$ \\
\hline$C(7)$ & $8445(1)$ & $1769(1)$ & $-3683(2)$ & $19(1)$ \\
\hline$C(8)$ & $8580(1)$ & $1876(1)$ & $-2441(2)$ & $16(1)$ \\
\hline$C(9)$ & $8595(1)$ & $1496(1)$ & $-1576(2)$ & $13(1)$ \\
\hline$C(10)$ & $8706(1)$ & $59(1)$ & $2029(2)$ & $15(1)$ \\
\hline
\end{tabular}




\begin{tabular}{|c|c|c|c|c|}
\hline $\mathrm{C}(11)$ & $8346(1)$ & $-195(1)$ & $2667(2)$ & $16(1)$ \\
\hline$C(12)$ & $8402(1)$ & $-354(1)$ & $3909(2)$ & $24(1)$ \\
\hline$C(13)$ & $8850(1)$ & $-283(1)$ & $4500(2)$ & $33(1)$ \\
\hline$C(14)$ & $9231(1)$ & $-57(1)$ & $3864(3)$ & $35(1)$ \\
\hline$C(15)$ & $9155(1)$ & $106(1)$ & $2640(3)$ & $22(1)$ \\
\hline$C(16)$ & $7982(1)$ & 1926(1) & $2154(2)$ & $12(1)$ \\
\hline $\mathrm{C}(17)$ & $8018(1)$ & $2760(1)$ & $2819(2)$ & $19(1)$ \\
\hline$C(18)$ & $7554(1)$ & 2531(1) & $3343(2)$ & $20(1)$ \\
\hline C(19) & $8764(1)$ & $2398(1)$ & 1902(2) & $13(1)$ \\
\hline $\mathrm{C}(20)$ & $9162(1)$ & $2402(1)$ & $2734(2)$ & $17(1)$ \\
\hline $\mathrm{C}(21)$ & $9634(1)$ & 2501(1) & $2342(2)$ & $22(1)$ \\
\hline $\mathrm{C}(22)$ & $9717(1)$ & $2617(1)$ & $1080(2)$ & $25(1)$ \\
\hline$C(23)$ & $9329(1)$ & $2648(1)$ & $223(2)$ & $21(1)$ \\
\hline $\mathrm{C}(24)$ & $8868(1)$ & 2541(1) & $659(2)$ & $15(1)$ \\
\hline$C(25)$ & $7104(1)$ & 1781(1) & $2738(2)$ & $14(1)$ \\
\hline$C(26)$ & $6983(1)$ & $1520(1)$ & $3834(2)$ & $18(1)$ \\
\hline$C(27)$ & $6533(1)$ & $1298(1)$ & $4027(2)$ & $22(1)$ \\
\hline$C(28)$ & $6182(1)$ & $1347(1)$ & $3076(3)$ & $25(1)$ \\
\hline$C(29)$ & $6274(1)$ & 1613(1) & $1980(2)$ & $21(1)$ \\
\hline$C(30)$ & $6733(1)$ & $1826(1)$ & $1847(2)$ & $16(1)$ \\
\hline $\mathrm{C}(31)$ & $7877(1)$ & $865(1)$ & $2569(2)$ & $14(1)$ \\
\hline$C(32)$ & $7428(1)$ & $576(1)$ & $2718(2)$ & $15(1)$ \\
\hline$C(33)$ & $7078(1)$ & $496(1)$ & $1765(2)$ & 19(1) \\
\hline$C(34)$ & $6656(1)$ & $225(1)$ & $2017(2)$ & $23(1)$ \\
\hline$C(35)$ & $6573(1)$ & $30(1)$ & $3231(3)$ & $26(1)$ \\
\hline$C(36)$ & $6917(1)$ & $97(1)$ & $4168(2)$ & $23(1)$ \\
\hline$C(37)$ & $7340(1)$ & $366(1)$ & $3931(2)$ & 19(1) \\
\hline $\mathrm{O}(41)$ & $6082(1)$ & $686(1)$ & $6620(2)$ & $59(1)$ \\
\hline
\end{tabular}




\begin{tabular}{lllll}
$\mathrm{C}(41)$ & $5702(2)$ & $996(2)$ & $7055(4)$ & $95(2)$ \\
$\mathrm{C}(42)$ & $5833(2)$ & $1174(1)$ & $8370(5)$ & $69(1)$ \\
$\mathrm{C}(43)$ & $6256(1)$ & $844(1)$ & $8749(4)$ & $57(1)$ \\
$\mathrm{C}(44)$ & $6474(1)$ & $703(2)$ & $7518(4)$ & $55(1)$ \\
& & & \\
$\mathrm{O}(1 \mathrm{~A})$ & 5000 & 10000 & 2500 & $272(8)$ \\
$\mathrm{C}(1 \mathrm{~A})$ & $5199(5)$ & $10028(12)$ & $1238(15)$ & $194(10)$ \\
$\mathrm{C}(2 \mathrm{~A})$ & $5058(19)$ & $10050(30)$ & $60(14)$ & $225(17)$ \\
\hline
\end{tabular}

Table 3. Selected bond lengths $[\AA]]$ and angles $\left[^{\circ}\right]$ for CCDC 279135.

\begin{tabular}{lllr}
\hline $\mathrm{Ru}(1)-\mathrm{C}(31)$ & $1.844(2)$ & $\mathrm{C}(31)-\mathrm{Ru}(1)-\mathrm{C}(1)$ & $96.44(9)$ \\
$\mathrm{Ru}(1)-\mathrm{C}(1)$ & $2.109(2)$ & $\mathrm{C}(31)-\mathrm{Ru}(1)-\mathrm{C}(16)$ & $94.35(8)$ \\
$\mathrm{Ru}(1)-\mathrm{C}(16)$ & $2.147(2)$ & $\mathrm{C}(1)-\operatorname{Ru}(1)-\mathrm{C}(16)$ & $168.92(8)$ \\
$\mathrm{Ru}(1)-\mathrm{Cl}(2)$ & $2.3985(5)$ & $\mathrm{C}(31)-\mathrm{Ru}(1)-\mathrm{Cl}(2)$ & $89.18(7)$ \\
$\mathrm{Ru}(1)-\mathrm{Cl}(1)$ & $2.4022(5)$ & $\mathrm{C}(1)-\operatorname{Ru}(1)-\mathrm{Cl}(2)$ & $89.78(6)$ \\
& & $\mathrm{C}(16)-\operatorname{Ru}(1)-\mathrm{Cl}(2)$ & $87.88(6)$ \\
& & $\mathrm{C}(31)-\operatorname{Ru}(1)-\mathrm{Cl}(1)$ & $101.73(7)$ \\
& & $\mathrm{C}(1)-\operatorname{Ru}(1)-\mathrm{Cl}(1)$ & $90.18(6)$ \\
& & $\mathrm{C}(16)-\operatorname{Ru}(1)-\mathrm{Cl}(1)$ & $90.07(6)$ \\
& & $\mathrm{Cl}(2)-\mathrm{Ru}(1)-\mathrm{Cl}(1)$ & $169.020(19)$ \\
& & & \\
$\mathrm{Ru}(1)-\mathrm{F}(2)$ & & & \\
\hline
\end{tabular}


Table 4. Bond lengths [ $[\AA]$ and angles [ $\left[^{\circ}\right]$ for CCDC 279135.

\begin{tabular}{|c|c|c|c|}
\hline $\mathrm{Ru}(1)-\mathrm{C}(31)$ & $1.844(2)$ & $C(4)-C(5)$ & $1.400(3)$ \\
\hline $\mathrm{Ru}(1)-\mathrm{C}(1)$ & $2.109(2)$ & $C(5)-C(6)$ & $1.379(3)$ \\
\hline $\mathrm{Ru}(1)-\mathrm{C}(16)$ & $2.147(2)$ & $C(6)-C(7)$ & $1.383(3)$ \\
\hline $\mathrm{Ru}(1)-\mathrm{Cl}(2)$ & $2.3985(5)$ & $\mathrm{C}(7)-\mathrm{C}(8)$ & $1.387(3)$ \\
\hline $\mathrm{Ru}(1)-\mathrm{Cl}(1)$ & $2.4022(5)$ & $\mathrm{C}(8)-\mathrm{C}(9)$ & $1.377(3)$ \\
\hline $\mathrm{F}(1)-\mathrm{C}(5)$ & $1.348(3)$ & $C(10)-C(11)$ & $1.375(3)$ \\
\hline $\mathrm{F}(2)-\mathrm{C}(9)$ & $1.367(2)$ & $\mathrm{C}(10)-\mathrm{C}(15)$ & $1.386(3)$ \\
\hline $\mathrm{F}(3)-\mathrm{C}(11)$ & $1.349(3)$ & $C(11)-C(12)$ & $1.384(3)$ \\
\hline $\mathrm{F}(4)-\mathrm{C}(15)$ & $1.343(3)$ & $C(12)-C(13)$ & $1.382(4)$ \\
\hline $\mathrm{F}(5)-\mathrm{C}(20)$ & $1.352(3)$ & $C(13)-C(14)$ & $1.379(4)$ \\
\hline $\mathrm{F}(6)-\mathrm{C}(24)$ & $1.362(2)$ & $\mathrm{C}(14)-\mathrm{C}(15)$ & $1.376(4)$ \\
\hline $\mathrm{F}(7)-\mathrm{C}(26)$ & $1.349(3)$ & $C(17)-C(18)$ & $1.512(3)$ \\
\hline $\mathrm{F}(8)-\mathrm{C}(30)$ & $1.351(3)$ & $C(19)-C(20)$ & $1.390(3)$ \\
\hline $\mathrm{N}(1)-\mathrm{C}(1)$ & $1.371(3)$ & $C(19)-C(24)$ & 1.393(3) \\
\hline $\mathrm{N}(1)-\mathrm{C}(4)$ & $1.409(3)$ & $C(20)-C(21)$ & $1.378(3)$ \\
\hline $\mathrm{N}(1)-\mathrm{C}(2)$ & $1.497(3)$ & $C(21)-C(22)$ & $1.382(4)$ \\
\hline $\mathrm{N}(2)-\mathrm{C}(1)$ & $1.341(3)$ & $C(22)-C(23)$ & $1.391(4)$ \\
\hline $\mathrm{N}(2)-\mathrm{C}(10)$ & $1.425(3)$ & $C(23)-C(24)$ & $1.367(3)$ \\
\hline $\mathrm{N}(2)-\mathrm{C}(3)$ & $1.475(3)$ & $C(25)-C(30)$ & $1.383(3)$ \\
\hline $\mathrm{N}(3)-\mathrm{C}(16)$ & $1.358(3)$ & $C(25)-C(26)$ & $1.394(3)$ \\
\hline $\mathrm{N}(3)-\mathrm{C}(19)$ & $1.414(3)$ & $C(26)-C(27)$ & $1.380(3)$ \\
\hline $\mathrm{N}(3)-\mathrm{C}(17)$ & $1.480(3)$ & $C(27)-C(28)$ & $1.390(4)$ \\
\hline $\mathrm{N}(4)-\mathrm{C}(16)$ & $1.347(3)$ & $C(28)-C(29)$ & $1.385(4)$ \\
\hline $\mathrm{N}(4)-\mathrm{C}(25)$ & $1.418(3)$ & $C(29)-C(30)$ & $1.383(3)$ \\
\hline $\mathrm{N}(4)-\mathrm{C}(18)$ & $1.482(3)$ & $\mathrm{C}(31)-\mathrm{C}(32)$ & $1.461(3)$ \\
\hline$C(2)-C(3)$ & $1.495(3)$ & $C(32)-C(33)$ & $1.399(3)$ \\
\hline $\mathrm{C}(4)-\mathrm{C}(9)$ & $1.386(3)$ & $C(32)-C(37)$ & $1.419(3)$ \\
\hline
\end{tabular}




\begin{tabular}{|c|c|c|c|}
\hline$C(33)-C(34)$ & $1.391(3)$ & $\mathrm{C}(1)-\mathrm{N}(1)-\mathrm{C}(4)$ & $127.52(18)$ \\
\hline C(34)-C(35) & $1.401(4)$ & $\mathrm{C}(1)-\mathrm{N}(1)-\mathrm{C}(2)$ & $112.61(18)$ \\
\hline$C(35)-C(36)$ & $1.373(4)$ & $\mathrm{C}(4)-\mathrm{N}(1)-\mathrm{C}(2)$ & $117.45(17)$ \\
\hline $\mathrm{C}(36)-\mathrm{C}(37)$ & $1.386(3)$ & $\mathrm{C}(1)-\mathrm{N}(2)-\mathrm{C}(10)$ & $127.43(18)$ \\
\hline $\mathrm{O}(41)-\mathrm{C}(41)$ & $1.411(5)$ & $\mathrm{C}(1)-\mathrm{N}(2)-\mathrm{C}(3)$ & $114.83(18)$ \\
\hline $\mathrm{O}(41)-\mathrm{C}(44)$ & $1.427(4)$ & $\mathrm{C}(10)-\mathrm{N}(2)-\mathrm{C}(3)$ & $117.72(18)$ \\
\hline $\mathrm{C}(41)-\mathrm{C}(42)$ & $1.508(6)$ & $\mathrm{C}(16)-\mathrm{N}(3)-\mathrm{C}(19)$ & $128.97(18)$ \\
\hline $\mathrm{C}(42)-\mathrm{C}(43)$ & $1.515(5)$ & $\mathrm{C}(16)-\mathrm{N}(3)-\mathrm{C}(17)$ & $113.64(17)$ \\
\hline$C(43)-C(44)$ & $1.475(5)$ & $\mathrm{C}(19)-\mathrm{N}(3)-\mathrm{C}(17)$ & $116.01(17)$ \\
\hline $\mathrm{O}(1 \mathrm{~A})-\mathrm{C}(1 \mathrm{~A}) \# 1$ & $1.435(17)$ & $\mathrm{C}(16)-\mathrm{N}(4)-\mathrm{C}(25)$ & $129.15(18)$ \\
\hline $\mathrm{O}(1 \mathrm{~A})-\mathrm{C}(1 \mathrm{~A})$ & $1.435(17)$ & $\mathrm{C}(16)-\mathrm{N}(4)-\mathrm{C}(18)$ & $113.82(18)$ \\
\hline $\mathrm{O}(1 \mathrm{~A})-\mathrm{C}(1 \mathrm{~A}) \# 2$ & $1.435(17)$ & $\mathrm{C}(25)-\mathrm{N}(4)-\mathrm{C}(18)$ & $116.42(17)$ \\
\hline $\mathrm{O}(1 \mathrm{~A})-\mathrm{C}(1 \mathrm{~A}) \# 3$ & $1.435(17)$ & $\mathrm{N}(2)-\mathrm{C}(1)-\mathrm{N}(1)$ & $105.76(18)$ \\
\hline$C(1 \mathrm{~A})-\mathrm{C}(1 \mathrm{~A}) \# 3$ & $1.09(3)$ & $\mathrm{N}(2)-\mathrm{C}(1)-\mathrm{Ru}(1)$ & $129.19(15)$ \\
\hline$C(1 \mathrm{~A})-\mathrm{C}(2 \mathrm{~A})$ & $1.30(2)$ & $\mathrm{N}(1)-\mathrm{C}(1)-\mathrm{Ru}(1)$ & $125.00(15)$ \\
\hline$C(1 A)-C(2 A) \# 3$ & $1.44(3)$ & $\mathrm{C}(3)-\mathrm{C}(2)-\mathrm{N}(1)$ & $102.48(18)$ \\
\hline$C(2 A)-C(2 A) \# 3$ & $0.43(14)$ & $\mathrm{N}(2)-\mathrm{C}(3)-\mathrm{C}(2)$ & $102.57(18)$ \\
\hline \multirow[t]{2}{*}{$C(2 A)-C(1 A) \# 3$} & $1.44(3)$ & $C(9)-C(4)-C(5)$ & $114.75(18)$ \\
\hline & & $\mathrm{C}(9)-\mathrm{C}(4)-\mathrm{N}(1)$ & $123.90(18)$ \\
\hline $\mathrm{C}(31)-\mathrm{Ru}(1)-\mathrm{C}(1)$ & $96.44(9)$ & $\mathrm{C}(5)-\mathrm{C}(4)-\mathrm{N}(1)$ & $121.23(19)$ \\
\hline $\mathrm{C}(31)-\mathrm{Ru}(1)-\mathrm{C}(16)$ & $94.35(8)$ & $\mathrm{F}(1)-\mathrm{C}(5)-\mathrm{C}(6)$ & $117.96(19)$ \\
\hline $\mathrm{C}(1)-\mathrm{Ru}(1)-\mathrm{C}(16)$ & $168.92(8)$ & $\mathrm{F}(1)-\mathrm{C}(5)-\mathrm{C}(4)$ & 118.91(19) \\
\hline $\mathrm{C}(31)-\mathrm{Ru}(1)-\mathrm{Cl}(2)$ & $89.18(7)$ & $C(6)-C(5)-C(4)$ & $123.1(2)$ \\
\hline $\mathrm{C}(1)-\mathrm{Ru}(1)-\mathrm{Cl}(2)$ & $89.78(6)$ & $C(5)-C(6)-C(7)$ & $119.1(2)$ \\
\hline $\mathrm{C}(16)-\mathrm{Ru}(1)-\mathrm{Cl}(2)$ & $87.88(6)$ & $C(6)-C(7)-C(8)$ & $120.3(2)$ \\
\hline $\mathrm{C}(31)-\mathrm{Ru}(1)-\mathrm{Cl}(1)$ & $101.73(7)$ & $\mathrm{C}(9)-\mathrm{C}(8)-\mathrm{C}(7)$ & $118.2(2)$ \\
\hline $\mathrm{C}(1)-\mathrm{Ru}(1)-\mathrm{Cl}(1)$ & $90.18(6)$ & $\mathrm{F}(2)-\mathrm{C}(9)-\mathrm{C}(8)$ & $118.67(18)$ \\
\hline $\mathrm{C}(16)-\mathrm{Ru}(1)-\mathrm{Cl}(1)$ & $90.07(6)$ & $\mathrm{F}(2)-\mathrm{C}(9)-\mathrm{C}(4)$ & $116.90(17)$ \\
\hline $\mathrm{Cl}(2)-\mathrm{Ru}(1)-\mathrm{Cl}(1)$ & $169.020(19)$ & $\mathrm{C}(8)-\mathrm{C}(9)-\mathrm{C}(4)$ & $124.43(18)$ \\
\hline
\end{tabular}




\begin{tabular}{|c|c|c|c|}
\hline$C(11)-C(10)-C(15)$ & $116.6(2)$ & $C(30)-C(25)-C(26)$ & $115.8(2)$ \\
\hline $\mathrm{C}(11)-\mathrm{C}(10)-\mathrm{N}(2)$ & $122.6(2)$ & $\mathrm{C}(30)-\mathrm{C}(25)-\mathrm{N}(4)$ & $121.5(2)$ \\
\hline $\mathrm{C}(15)-\mathrm{C}(10)-\mathrm{N}(2)$ & $120.1(2)$ & $\mathrm{C}(26)-\mathrm{C}(25)-\mathrm{N}(4)$ & $121.7(2)$ \\
\hline $\mathrm{F}(3)-\mathrm{C}(11)-\mathrm{C}(10)$ & $117.65(19)$ & $\mathrm{F}(7)-\mathrm{C}(26)-\mathrm{C}(27)$ & $118.7(2)$ \\
\hline $\mathrm{F}(3)-\mathrm{C}(11)-\mathrm{C}(12)$ & $119.7(2)$ & $\mathrm{F}(7)-\mathrm{C}(26)-\mathrm{C}(25)$ & $117.6(2)$ \\
\hline$C(10)-C(11)-C(12)$ & $122.6(2)$ & $\mathrm{C}(27)-\mathrm{C}(26)-\mathrm{C}(25)$ & $123.6(2)$ \\
\hline$C(13)-C(12)-C(11)$ & $118.5(2)$ & $C(26)-C(27)-C(28)$ & $117.5(2)$ \\
\hline$C(14)-C(13)-C(12)$ & $120.5(2)$ & $C(29)-C(28)-C(27)$ & $121.7(2)$ \\
\hline$C(15)-C(14)-C(13)$ & $119.0(2)$ & $\mathrm{C}(30)-\mathrm{C}(29)-\mathrm{C}(28)$ & $117.9(2)$ \\
\hline$F(4)-C(15)-C(14)$ & $119.3(2)$ & $\mathrm{F}(8)-\mathrm{C}(30)-\mathrm{C}(29)$ & $118.1(2)$ \\
\hline $\mathrm{F}(4)-\mathrm{C}(15)-\mathrm{C}(10)$ & $118.3(2)$ & $\mathrm{F}(8)-\mathrm{C}(30)-\mathrm{C}(25)$ & $118.23(19)$ \\
\hline$C(14)-C(15)-C(10)$ & $122.4(2)$ & $\mathrm{C}(29)-\mathrm{C}(30)-\mathrm{C}(25)$ & $123.5(2)$ \\
\hline $\mathrm{N}(4)-\mathrm{C}(16)-\mathrm{N}(3)$ & $105.25(18)$ & $\mathrm{C}(32)-\mathrm{C}(31)-\mathrm{Ru}(1)$ & $138.27(16)$ \\
\hline $\mathrm{N}(4)-\mathrm{C}(16)-\mathrm{Ru}(1)$ & $127.90(15)$ & $\mathrm{C}(33)-\mathrm{C}(32)-\mathrm{C}(37)$ & $117.9(2)$ \\
\hline $\mathrm{N}(3)-\mathrm{C}(16)-\mathrm{Ru}(1)$ & $126.80(15)$ & $\mathrm{C}(33)-\mathrm{C}(32)-\mathrm{C}(31)$ & $125.2(2)$ \\
\hline $\mathrm{N}(3)-\mathrm{C}(17)-\mathrm{C}(18)$ & $101.10(17)$ & $C(37)-C(32)-C(31)$ & $117.0(2)$ \\
\hline $\mathrm{N}(4)-\mathrm{C}(18)-\mathrm{C}(17)$ & $101.19(17)$ & $C(34)-C(33)-C(32)$ & $120.5(2)$ \\
\hline$C(20)-C(19)-C(24)$ & $115.5(2)$ & $C(33)-C(34)-C(35)$ & $120.6(2)$ \\
\hline $\mathrm{C}(20)-\mathrm{C}(19)-\mathrm{N}(3)$ & $121.58(19)$ & $C(36)-C(35)-C(34)$ & $119.5(2)$ \\
\hline $\mathrm{C}(24)-\mathrm{C}(19)-\mathrm{N}(3)$ & $122.32(19)$ & $C(35)-C(36)-C(37)$ & $120.6(2)$ \\
\hline $\mathrm{F}(5)-\mathrm{C}(20)-\mathrm{C}(21)$ & $119.4(2)$ & $C(36)-C(37)-C(32)$ & $120.9(2)$ \\
\hline $\mathrm{F}(5)-\mathrm{C}(20)-\mathrm{C}(19)$ & $118.0(2)$ & $\mathrm{C}(41)-\mathrm{O}(41)-\mathrm{C}(44)$ & $108.3(3)$ \\
\hline$C(21)-C(20)-C(19)$ & $122.6(2)$ & $\mathrm{O}(41)-\mathrm{C}(41)-\mathrm{C}(42)$ & $108.5(3)$ \\
\hline$C(20)-C(21)-C(22)$ & $118.9(2)$ & $C(41)-C(42)-C(43)$ & $103.3(3)$ \\
\hline$C(21)-C(22)-C(23)$ & $120.9(2)$ & $C(44)-C(43)-C(42)$ & $103.2(3)$ \\
\hline$C(24)-C(23)-C(22)$ & $117.8(2)$ & $\mathrm{O}(41)-\mathrm{C}(44)-\mathrm{C}(43)$ & $106.8(3)$ \\
\hline $\mathrm{F}(6)-\mathrm{C}(24)-\mathrm{C}(23)$ & $119.9(2)$ & $\mathrm{C}(1 \mathrm{~A}) \# 1-\mathrm{O}(1 \mathrm{~A})-\mathrm{C}(1 \mathrm{~A})$ & $148.8(8)$ \\
\hline $\mathrm{F}(6)-\mathrm{C}(24)-\mathrm{C}(19)$ & $116.1(2)$ & $\mathrm{C}(2 \mathrm{~A})-\mathrm{C}(1 \mathrm{~A})-\mathrm{O}(1 \mathrm{~A})$ & $141(2)$ \\
\hline$C(23)-C(24)-C(19)$ & $124.1(2)$ & $\mathrm{C}(2 \mathrm{~A}) \# 3-\mathrm{C}(1 \mathrm{~A})-\mathrm{O}(1 \mathrm{~A})$ & $127(2)$ \\
\hline
\end{tabular}


$\mathrm{C}(2 \mathrm{~A}) \# 3-\mathrm{C}(2 \mathrm{~A})-\mathrm{C}(1 \mathrm{~A}) \quad$ 100(7)

Symmetry transformations used to generate equivalent atoms:

$\# 1-\mathrm{y}+3 / 2, \mathrm{x}+1 / 2,-\mathrm{z}+1 / 2$

$\# 2 y-1 / 2,-x+3 / 2,-z+1 / 2$

$\# 3-x+1,-y+2, z$ 
Table 5. Anisotropic displacement parameters $\left(\AA^{2} \times 1^{4}\right)$ for CCDC 279135. The anisotropic displacement factor exponent takes the form: $-2 \pi^{2}\left[h^{2} a^{* 2} U 11+\ldots+2 h\right.$ $\left.\mathbf{k} \mathbf{a}^{*} \mathbf{b}^{*} \mathbf{U}^{12}\right]$

\begin{tabular}{|c|c|c|c|c|c|c|}
\hline & $\mathrm{U}^{11}$ & $\mathrm{U}^{22}$ & $\mathrm{U}^{33}$ & $U^{23}$ & $\mathrm{U}^{13}$ & $\mathrm{U}^{12}$ \\
\hline $\mathrm{Ru}(1)$ & $109(1)$ & $104(1)$ & $88(1)$ & $-6(1)$ & $1(1)$ & $14(1)$ \\
\hline $\mathrm{Cl}(1)$ & $138(2)$ & $189(3)$ & $132(2)$ & $10(2)$ & $-24(2)$ & $24(2)$ \\
\hline $\mathrm{Cl}(2)$ & $129(2)$ & $159(2)$ & $136(2)$ & $8(2)$ & $-29(2)$ & $8(2)$ \\
\hline $\mathrm{F}(1)$ & $489(9)$ & $206(7)$ & $180(7)$ & $-30(6)$ & $-63(6)$ & $-107(7)$ \\
\hline $\mathrm{F}(2)$ & 193(7) & $157(6)$ & $105(5)$ & $-7(5)$ & $-21(5)$ & $-6(5)$ \\
\hline $\mathrm{F}(3)$ & $202(7)$ & 209(7) & $351(8)$ & $-51(6)$ & $-31(6)$ & $-16(6)$ \\
\hline $\mathrm{F}(4)$ & $204(8)$ & $250(8)$ & $668(11)$ & $130(8)$ & $33(8)$ & $-29(6)$ \\
\hline $\mathrm{F}(5)$ & $281(8)$ & $327(8)$ & $122(6)$ & $37(5)$ & $-42(5)$ & $-43(6)$ \\
\hline $\mathrm{F}(6)$ & $311(8)$ & $205(7)$ & $143(6)$ & $11(5)$ & $-91(6)$ & $38(6)$ \\
\hline $\mathrm{F}(7)$ & $284(8)$ & $310(8)$ & $166(6)$ & $-34(6)$ & $-54(6)$ & $36(6)$ \\
\hline $\mathrm{F}(8)$ & $240(7)$ & $223(7)$ & $190(6)$ & $20(6)$ & $-8(6)$ & $26(6)$ \\
\hline $\mathrm{N}(1)$ & $225(10)$ & $108(8)$ & $126(8)$ & $13(6)$ & $37(7)$ & $35(7)$ \\
\hline $\mathrm{N}(2)$ & $270(11)$ & 141(9) & $120(8)$ & $21(7)$ & $49(7)$ & $61(8)$ \\
\hline $\mathrm{N}(3)$ & $127(9)$ & $126(9)$ & $151(8)$ & $-28(7)$ & $14(7)$ & $11(6)$ \\
\hline $\mathrm{N}(4)$ & $110(9)$ & 116(9) & $230(9)$ & $-66(7)$ & $26(7)$ & $3(7)$ \\
\hline $\mathrm{C}(1)$ & $155(10)$ & 99(9) & 122(9) & $4(7)$ & $-1(8)$ & $2(8)$ \\
\hline$C(2)$ & $402(14)$ & $167(11)$ & 151(10) & $-14(9)$ & $77(11)$ & $103(10)$ \\
\hline$C(3)$ & $680(20)$ & 181(13) & $183(12)$ & $33(10)$ & $137(13)$ & $212(13)$ \\
\hline$C(4)$ & $152(10)$ & $140(10)$ & $108(9)$ & $22(7)$ & $19(7)$ & $11(8)$ \\
\hline$C(5)$ & $238(12)$ & $157(11)$ & $136(10)$ & $-24(8)$ & $-30(8)$ & $-23(9)$ \\
\hline$C(6)$ & $253(13)$ & $254(13)$ & 104(9) & $14(9)$ & $-20(8)$ & $15(9)$ \\
\hline$C(7)$ & $228(11)$ & 194(10) & 152(9) & $55(10)$ & $13(10)$ & $24(8)$ \\
\hline$C(8)$ & $176(11)$ & $136(10)$ & $156(10)$ & $12(8)$ & $0(8)$ & $0(8)$ \\
\hline $\mathrm{C}(9)$ & $135(10)$ & $172(10)$ & $76(9)$ & $-9(7)$ & $-4(7)$ & $12(8)$ \\
\hline
\end{tabular}




\begin{tabular}{|c|c|c|c|c|c|c|}
\hline$C(10)$ & $188(11)$ & $124(10)$ & 131(9) & $23(7)$ & $0(8)$ & $33(8)$ \\
\hline $\mathrm{C}(11)$ & $178(11)$ & $138(10)$ & $174(10)$ & $-12(8)$ & $-27(8)$ & $6(8)$ \\
\hline$C(12)$ & $386(14)$ & $137(11)$ & 199(12) & $25(8)$ & $84(10)$ & $-25(10)$ \\
\hline$C(13)$ & $600(20)$ & $221(13)$ & $154(11)$ & 44(9) & $-106(12)$ & $37(13)$ \\
\hline$C(14)$ & $391(16)$ & $245(13)$ & $399(17)$ & $53(12)$ & $-262(13)$ & $9(11)$ \\
\hline$C(15)$ & $175(11)$ & $133(11)$ & $365(14)$ & $56(10)$ & $-24(10)$ & $-2(9)$ \\
\hline$C(16)$ & $129(10)$ & $130(10)$ & 106(9) & $2(7)$ & $-19(7)$ & $18(8)$ \\
\hline $\mathrm{C}(17)$ & $184(11)$ & $150(11)$ & $234(11)$ & $-65(8)$ & 22(9) & $27(8)$ \\
\hline$C(18)$ & $159(11)$ & $170(11)$ & $258(12)$ & $-115(9)$ & $24(8)$ & $0(9)$ \\
\hline$C(19)$ & $129(10)$ & $100(10)$ & $147(9)$ & $-1(7)$ & $8(7)$ & $9(7)$ \\
\hline$C(20)$ & 198(11) & $183(11)$ & $125(9)$ & $13(8)$ & $-18(8)$ & $-6(9)$ \\
\hline $\mathrm{C}(21)$ & $143(11)$ & $220(12)$ & $303(13)$ & $-8(10)$ & $-37(9)$ & $-18(9)$ \\
\hline$C(22)$ & $183(11)$ & $212(12)$ & $353(15)$ & $-55(10)$ & $86(10)$ & $-56(9)$ \\
\hline$C(23)$ & $276(13)$ & $174(11)$ & $182(11)$ & $-15(9)$ & $87(9)$ & $-18(9)$ \\
\hline$C(24)$ & $188(11)$ & $122(10)$ & $147(10)$ & $-2(8)$ & $-50(8)$ & $15(8)$ \\
\hline$C(25)$ & $119(10)$ & $117(10)$ & $185(10)$ & $-67(8)$ & $8(8)$ & $10(8)$ \\
\hline$C(26)$ & 199(11) & $189(11)$ & $158(11)$ & $-37(8)$ & $-8(8)$ & $47(8)$ \\
\hline $\mathrm{C}(27)$ & $245(12)$ & $190(12)$ & $213(11)$ & $-7(9)$ & 73(9) & 19(9) \\
\hline$C(28)$ & $169(12)$ & $215(13)$ & $362(14)$ & $-27(11)$ & $76(10)$ & $-26(9)$ \\
\hline $\mathrm{C}(29)$ & 141(11) & $238(13)$ & $253(12)$ & $-35(9)$ & $-25(9)$ & $3(9)$ \\
\hline$C(30)$ & $177(11)$ & $132(10)$ & $178(10)$ & $-22(8)$ & $32(8)$ & $6(8)$ \\
\hline $\mathrm{C}(31)$ & $142(10)$ & $168(11)$ & $115(9)$ & $-30(8)$ & $-14(8)$ & $31(8)$ \\
\hline$C(32)$ & $149(10)$ & $119(10)$ & $170(10)$ & $-15(8)$ & $20(8)$ & $25(8)$ \\
\hline $\mathrm{C}(33)$ & 201(12) & $155(11)$ & 199(10) & $-33(8)$ & $2(8)$ & $-15(9)$ \\
\hline$C(34)$ & $213(12)$ & $217(12)$ & $265(12)$ & $-24(10)$ & $-36(10)$ & $-40(10)$ \\
\hline$C(35)$ & $216(13)$ & $175(12)$ & $381(14)$ & $2(10)$ & $59(10)$ & $-46(10)$ \\
\hline$C(36)$ & $306(14)$ & $169(12)$ & $225(11)$ & $44(9)$ & $75(10)$ & $-36(10)$ \\
\hline$C(37)$ & 208(11) & 207(11) & 151(11) & $8(8)$ & $32(8)$ & $1(9)$ \\
\hline
\end{tabular}




\begin{tabular}{lrrrrrr}
$\mathrm{O}(41)$ & $638(17)$ & $715(18)$ & $419(15)$ & $74(12)$ & $-17(12)$ & $95(14)$ \\
$\mathrm{C}(41)$ & $660(30)$ & $1520(50)$ & $660(30)$ & $310(30)$ & $60(20)$ & $610(30)$ \\
$\mathrm{C}(42)$ & $610(30)$ & $450(20)$ & $1010(40)$ & $-150(20)$ & $-50(20)$ & $147(19)$ \\
$\mathrm{C}(43)$ & $540(20)$ & $670(20)$ & $500(20)$ & $-140(20)$ & $-90(20)$ & $21(18)$ \\
$\mathrm{C}(44)$ & $410(20)$ & $640(20)$ & $580(20)$ & $140(20)$ & $-47(18)$ & $104(17)$ \\
& & & & & \\
$\mathrm{O}(1 \mathrm{~A})$ & $3090(150)$ & $3090(150)$ & $1980(170)$ & 0 & 0 & 0 \\
$\mathrm{C}(1 \mathrm{~A})$ & $1300(150)$ & $3600(300)$ & $930(90)$ & $490(190)$ & $-230(100)$ & $300(200)$ \\
$\mathrm{C}(2 \mathrm{~A})$ & $1600(300)$ & $4000(400)$ & $1180(110)$ & $-600(300)$ & $700(200)$ & $-900(200)$ \\
& & & & & & \\
\hline
\end{tabular}

Table 6. Torsion angles [ $\left[^{\circ}\right]$ for CCDC 279135.

\begin{tabular}{lc}
\hline $\mathrm{C}(1)-\mathrm{N}(1)-\mathrm{C}(4)-\mathrm{C}(9)$ & $33.0(3)$ \\
$\mathrm{C}(1)-\mathrm{N}(2)-\mathrm{C}(10)-\mathrm{C}(11)$ & $91.2(3)$ \\
$\mathrm{C}(16)-\mathrm{N}(3)-\mathrm{C}(19)-\mathrm{C}(24)$ & $78.8(3)$ \\
$\mathrm{C}(16)-\mathrm{N}(4)-\mathrm{C}(25)-\mathrm{C}(26)$ & $95.2(3)$ \\
& \\
$\mathrm{N}(2)-\mathrm{C}(1)-\mathrm{Ru}(1)-\mathrm{C}(31)$ & $-36.8(2)$ \\
$\mathrm{N}(4)-\mathrm{C}(16)-\mathrm{Ru}(1)-\mathrm{C}(31)$ & $-43.1(2)$ \\
\end{tabular}

Symmetry transformations used to generate equivalent atoms:

$\# 1-y+3 / 2, x+1 / 2,-z+1 / 2 \quad \# 2 y-1 / 2,-x+3 / 2,-z+1 / 2$

$\# 3-x+1,-y+2, z$ 


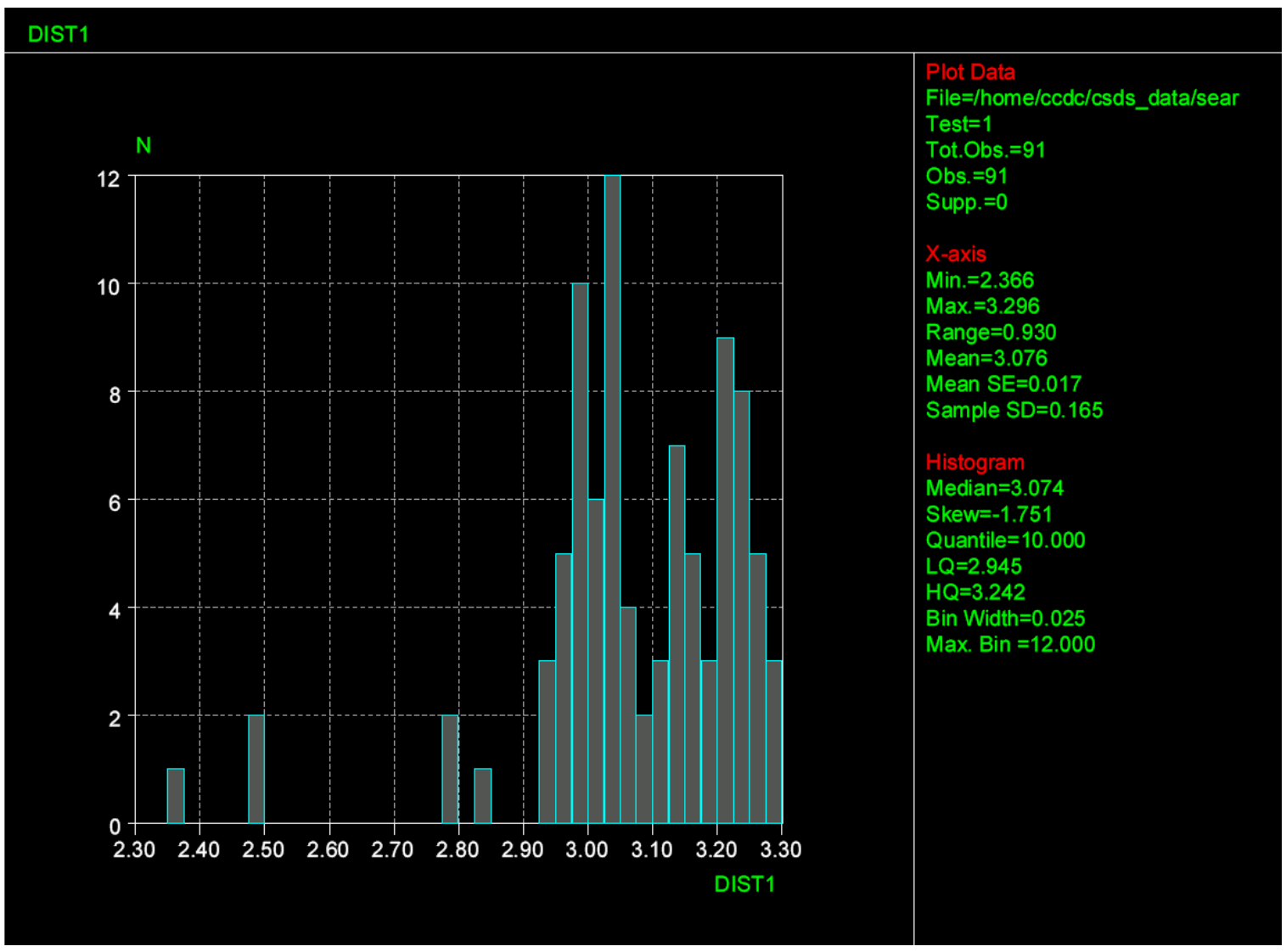

The histogram shows the occurrences of complexes from the CCDC, as a function of Ru$\mathrm{F}$ distances, in which the fluorine is attached to a carbon atom. It is evident that the $\mathrm{Ru}-\mathrm{F}$ distances in S6 and S7 are among the shortest ever observed for such interactions. 


\section{NMR Spectra}

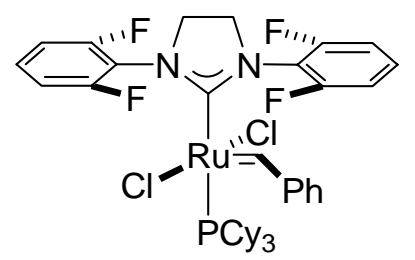

${ }^{1} \mathrm{H}$ NMR

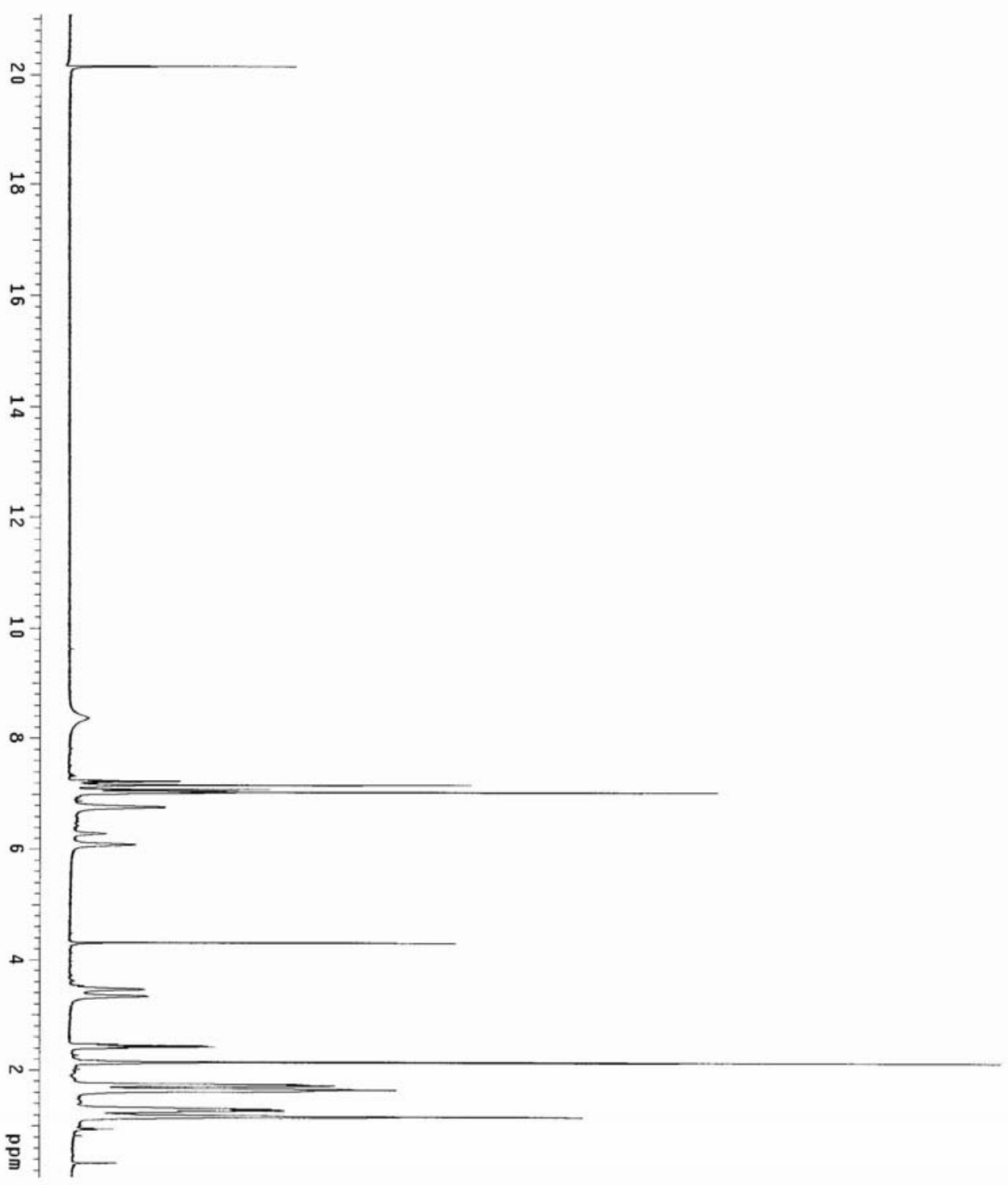


${ }^{13} \mathrm{C}$ NMR

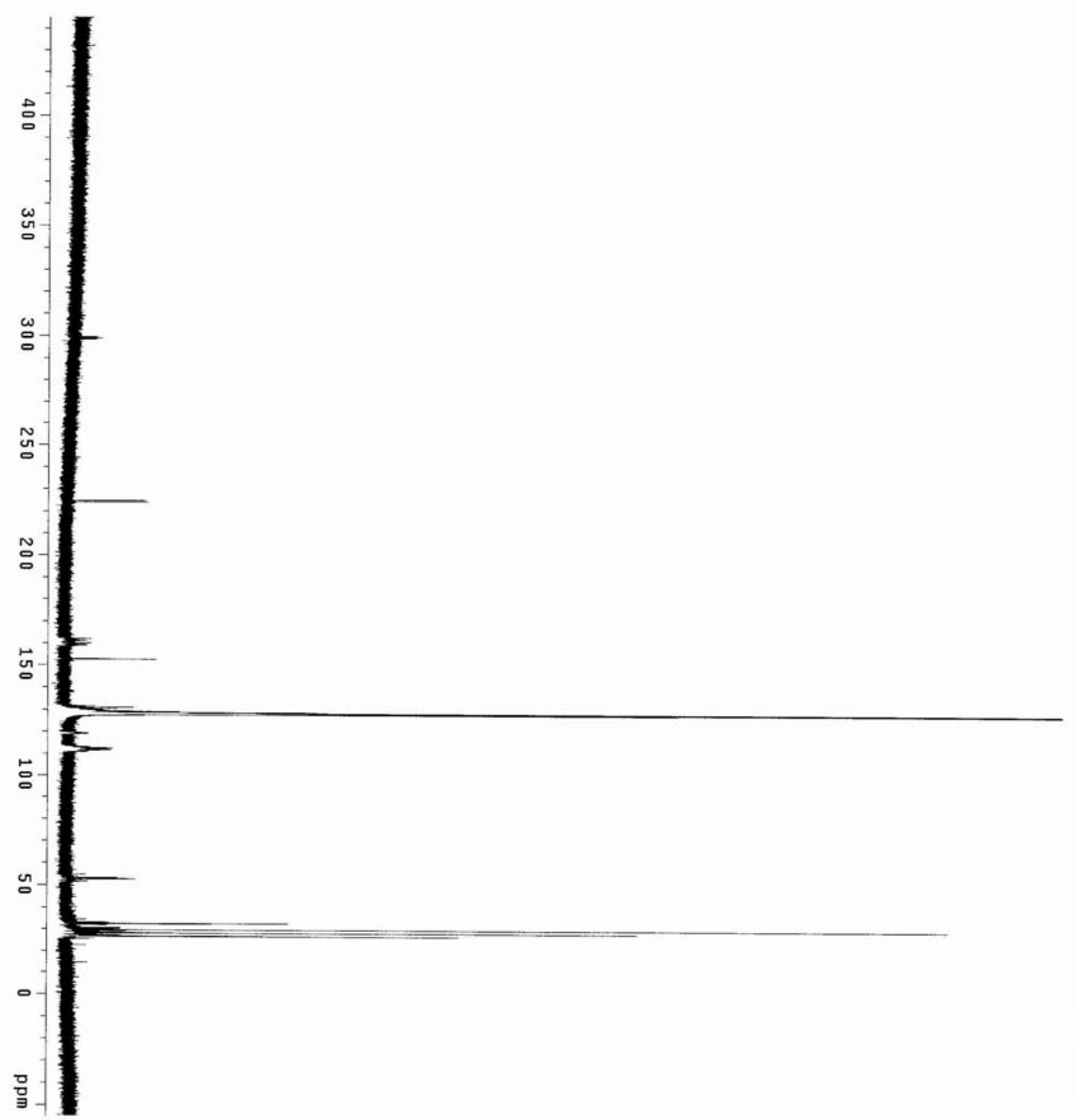


${ }^{31}$ P NMR

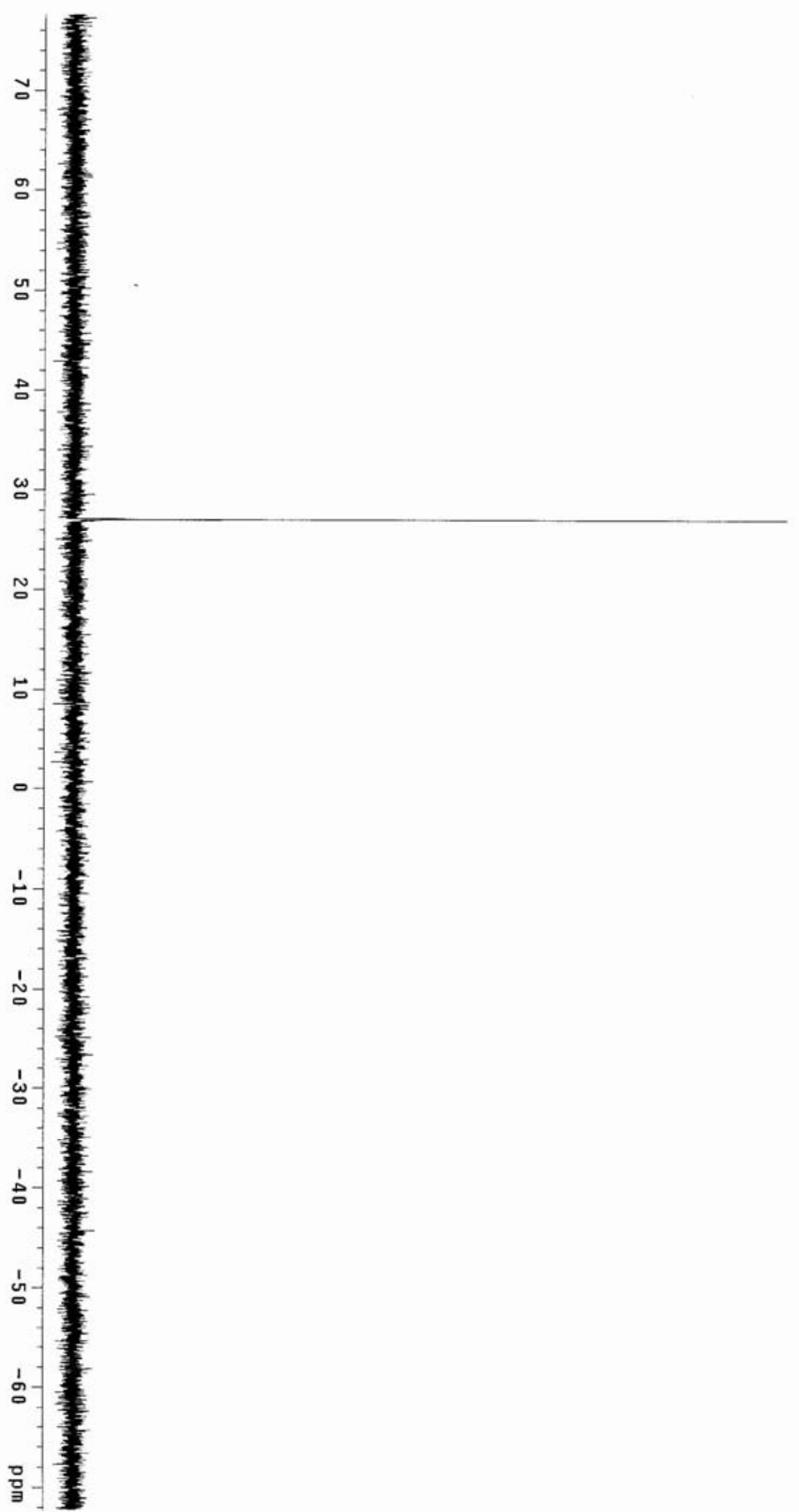


${ }^{19}$ F NMR

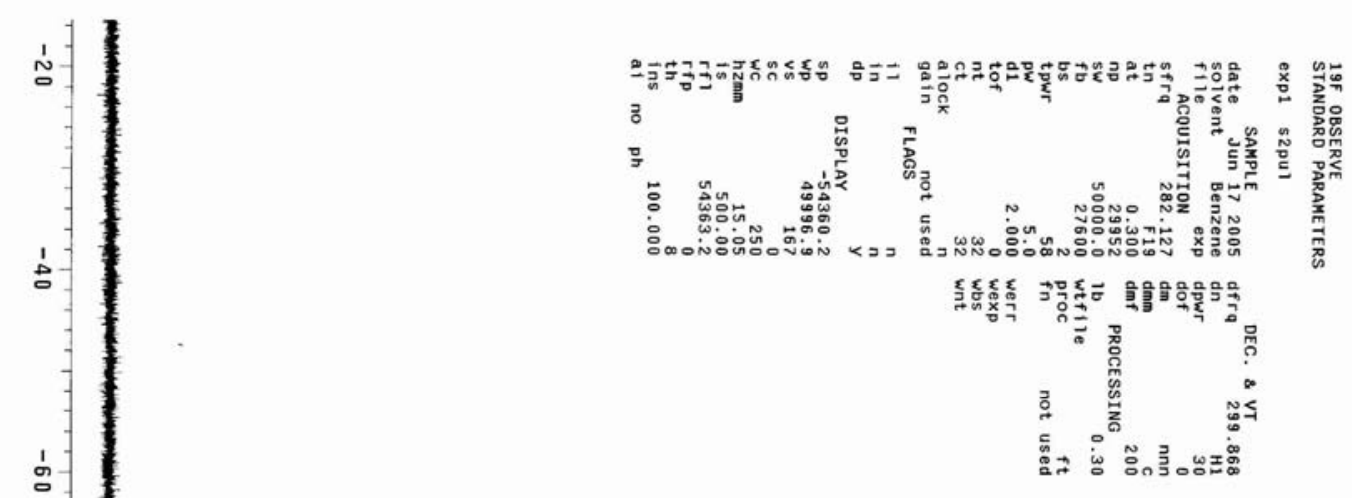

$\stackrel{1}{a}$

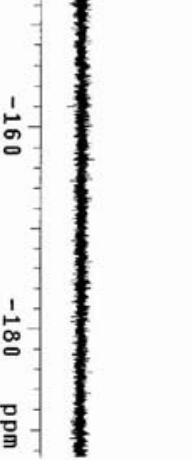




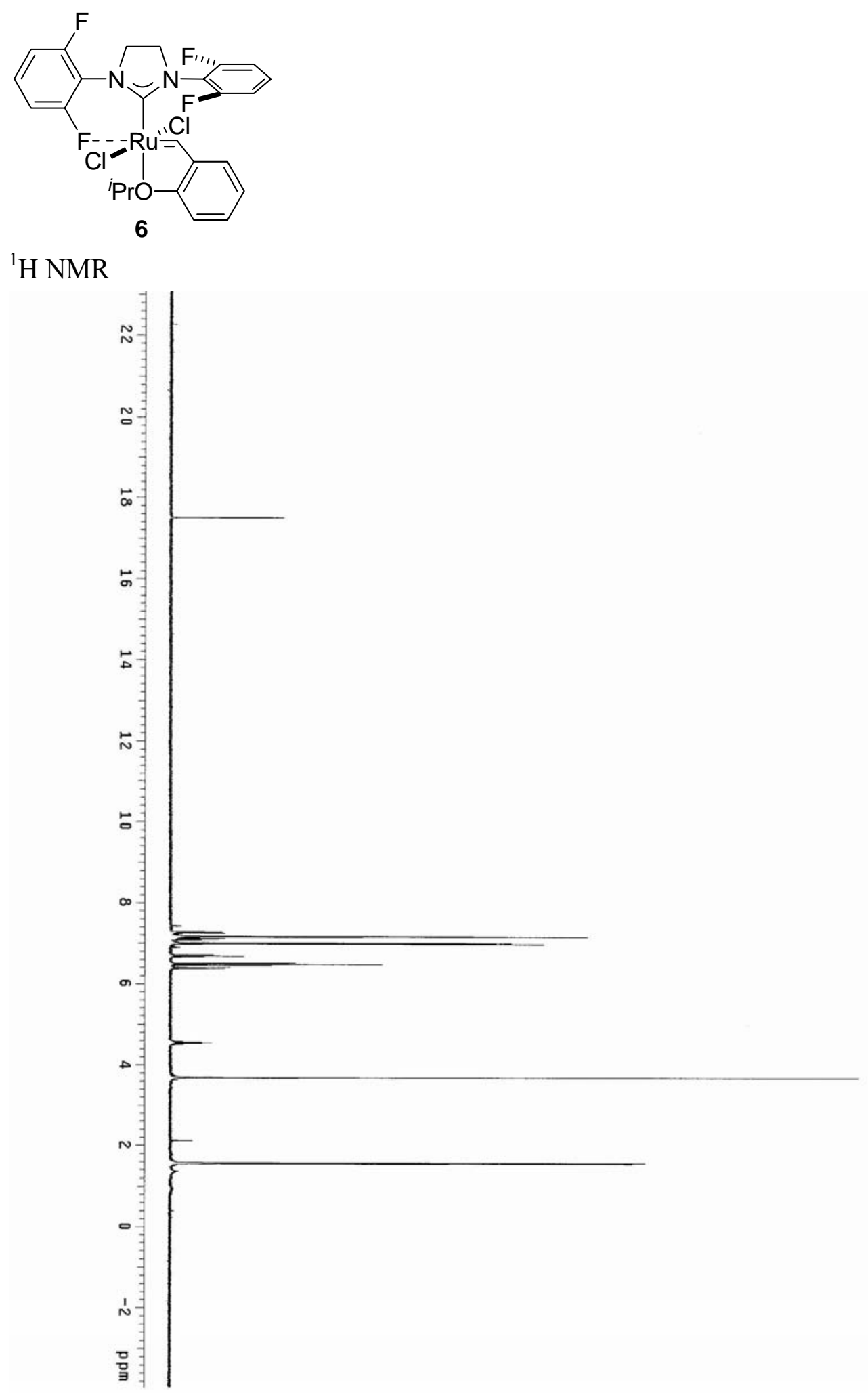


${ }^{13} \mathrm{C}$ NMR

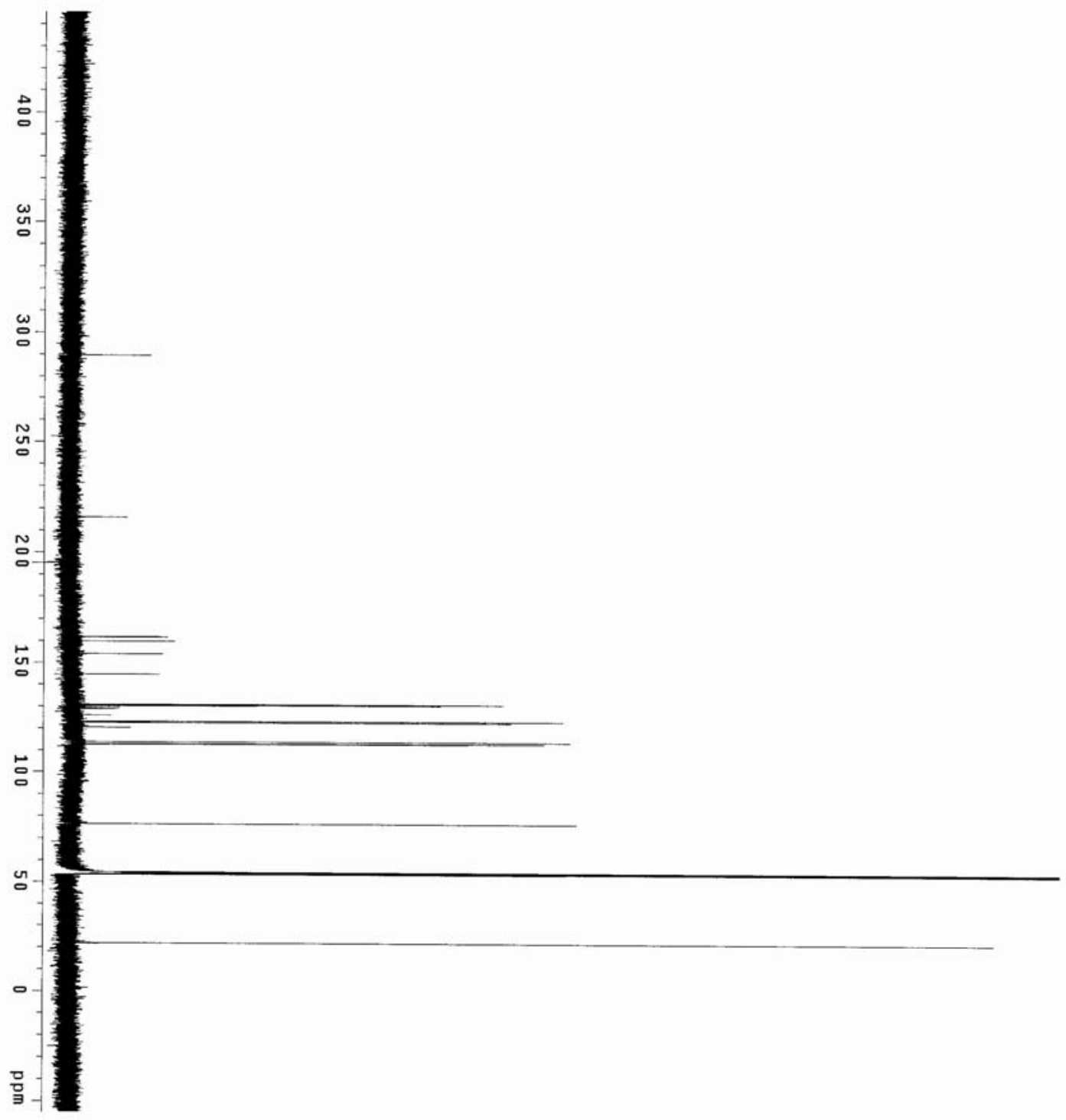


${ }^{19} \mathrm{~F}$ NMR

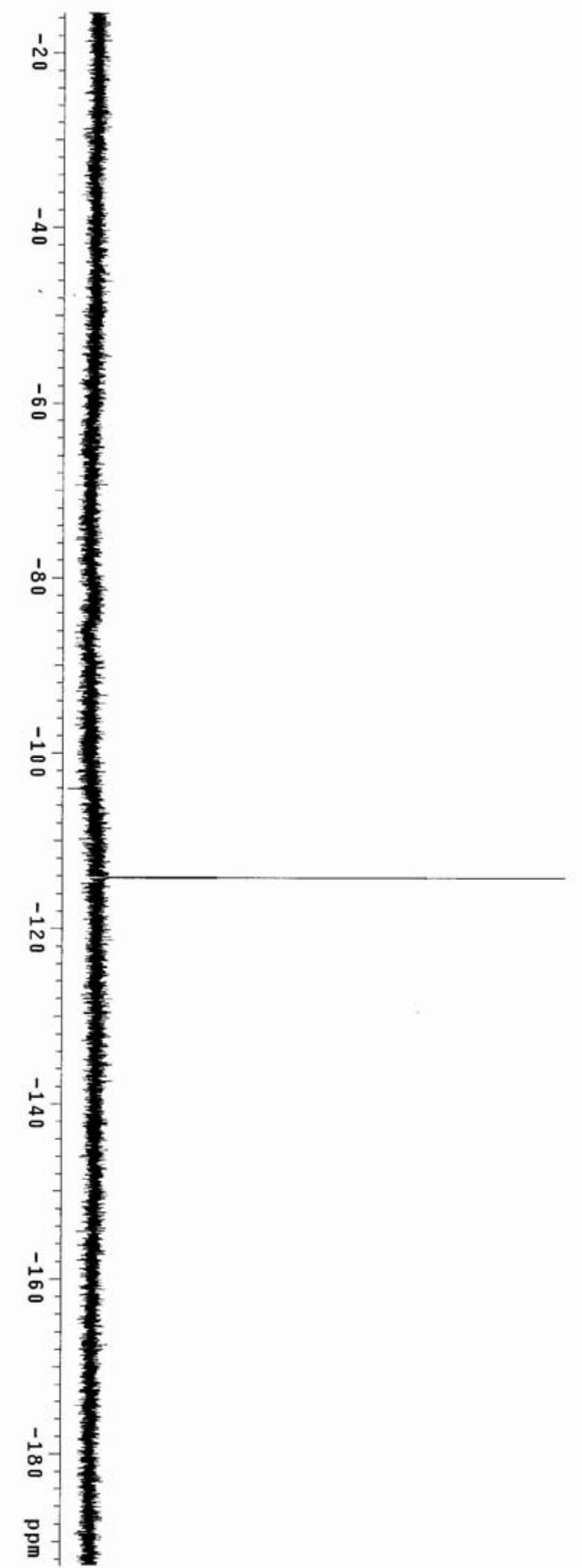




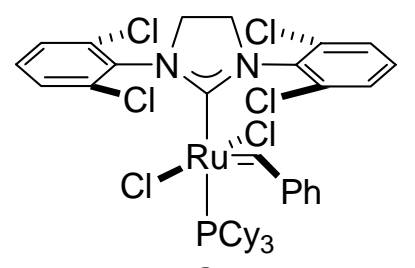

S3

${ }^{1} \mathrm{H}$ NMR

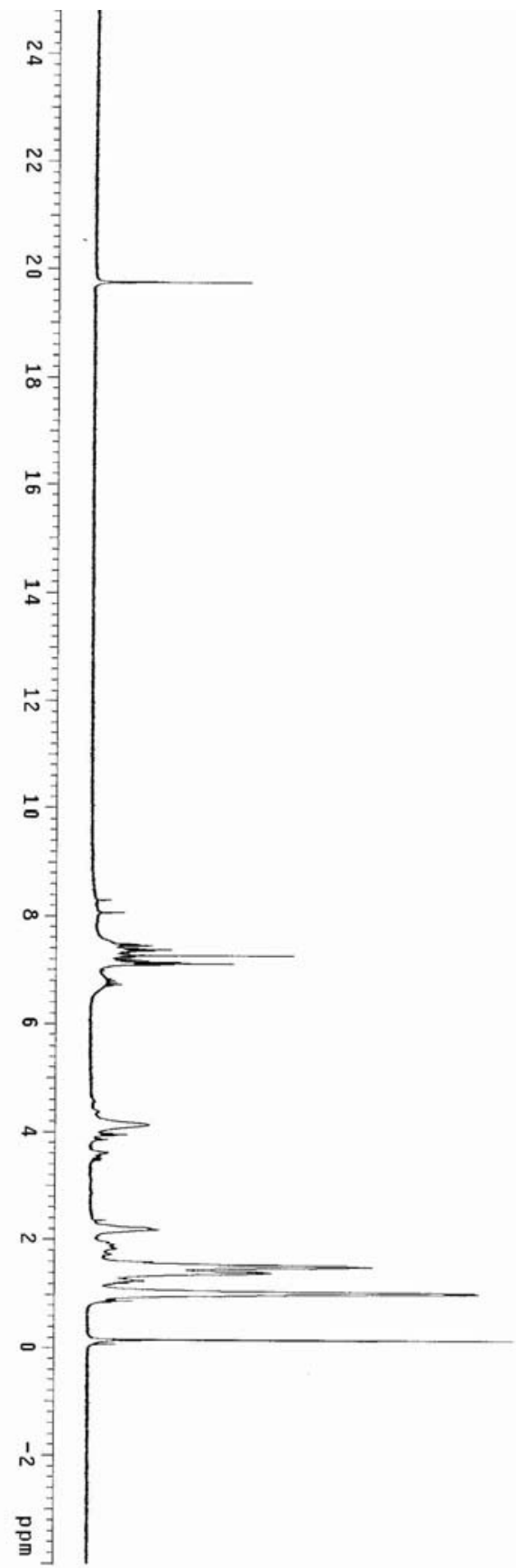


${ }^{31}$ P NMR

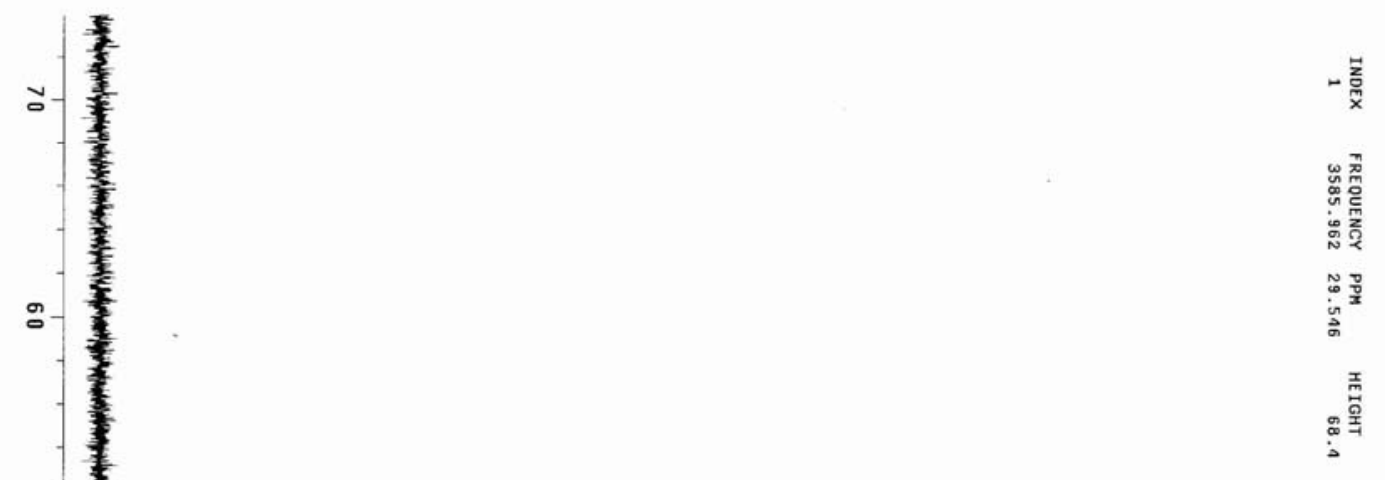

ज-

-

$\circ$

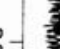

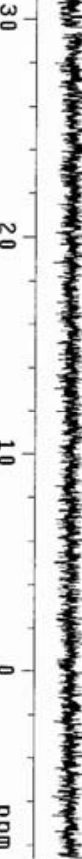




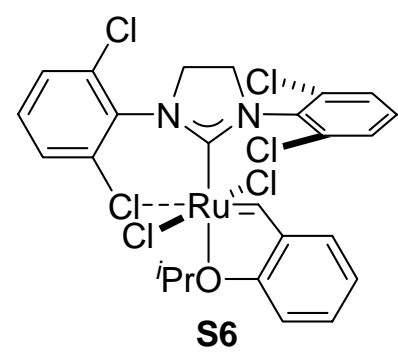

${ }^{1} \mathrm{H}$ NMR

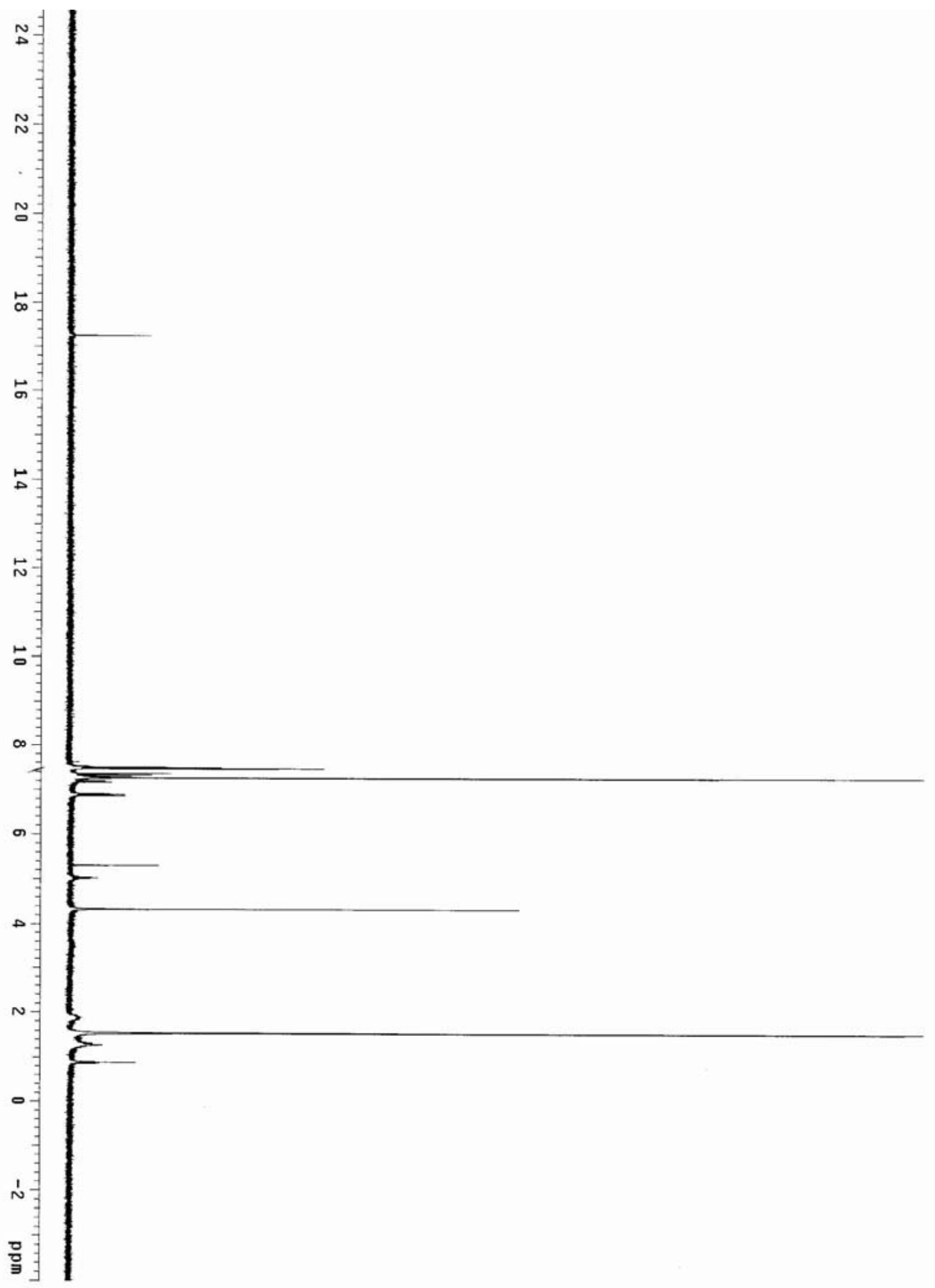

